\title{
Photoaktivierungsfähige Rhodamine als Bio-Calcium-Sensoren und Markierungen für Tetracystein-Tags in Proteinen
}

\author{
DISSERTATION
}

zur Erlangung des mathematisch-naturwissenschaftlichen Doktorgrades

"Doctor rerum naturalium"

der Georg-August-Universität Göttingen

vorgelegt von

Sergey Yan

aus Leningrad (UdSSR)

Göttingen, 2010 
Referent: Prof. Dr. Armin de Meijere

Korreferent: Prof. Dr. Stefan W. Hell

Tag der mündlichen Prüfung: 28. Januar 2011 
Die vorliegende Arbeit wurde in der Zeit von September 2006 bis November 2010 im Max-Planck-Institut für biophysikalische Chemie in Göttingen angefertigt.

Hiermit möchte ich für die Überlassung des interessanten Themas und große Unterstützung während meiner Promotionszeit Herrn Prof. Dr. Armin de Meijere, Herrn Prof. Dr. Stefan W. Hell und Herrn Dr. Vladimir N. Belov ganz herzlich danken. 
to my family 


\section{Table of Contents}

Abbreviations.............................................................................................................................

Preface........................................................................................................................ 10

Chapter 1. Fluorescent $\mathrm{Ca}^{2+}$ sensors for imaging calcium distributions in biomatter ............................................................................. 13

1. Fluorescence and principles of fluorescence microscopy....................................13

2. Fluorescent indicators for calcium cations.......................................................18

2.1. Main photophysical principles of ion sensing by fluorescent indicators and determination of calcium concentrations from the spectroscopic data............................ 18

2.2. Diverse coordination moieties in fluorescent $\mathrm{Ca}^{2+}$ indicators ............................. 21

2.3. Early steps in the creation of highly sensitive fluorescent calcium indicators for

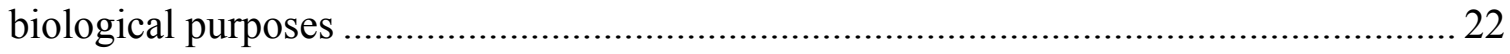

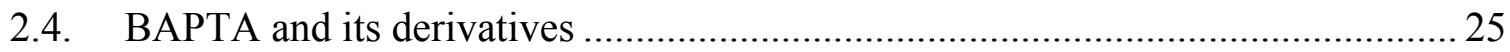

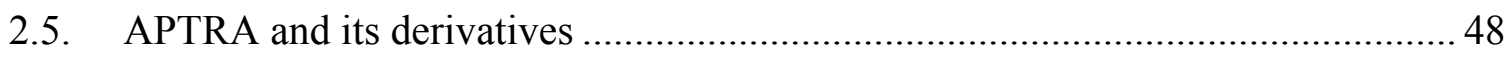

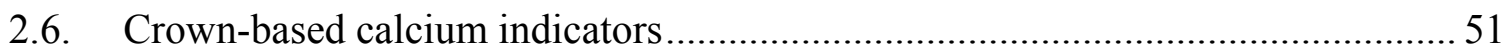

2.7. Calixarene hosts as coordination moieties for calcium sensors.............................. 58

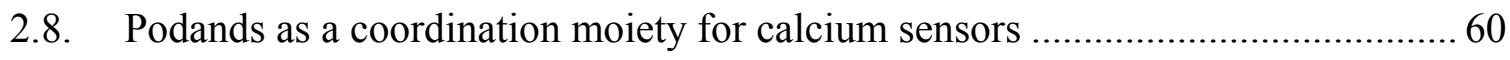

Chapter 2. Synthesis and properties of new fluorescent calcium sensors..... 63

1. Search for an optimal coordinative part and its position within the phenylxanthene backbone...............................................................................63

2. Model compounds ...............................................................................................................70

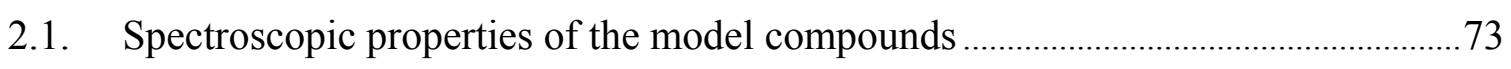

3. Synthesis of rhodole derivatives with a crown-ether moiety fused with the

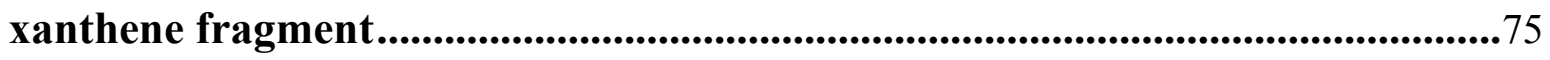

3.1. Synthesis of the phenol building blocks via the unfunctionalized crown ether.... 75

3.2. Attempted synthesis of phenol building blocks via the crown ether prepared from

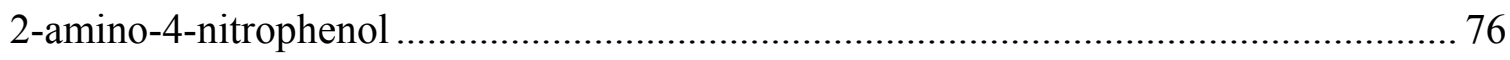

3.3. Synthesis of the phenol building blocks by a ring-closing reaction of a

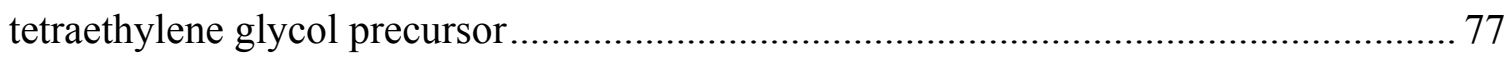

3.4. Spectroscopic properties of the target compounds in the presence of $\mathrm{Ca}^{2+}$ ions ... 82

4. Synthesis of $N, N, N^{\prime}, N^{\prime}$-tetramethylrhodamine (TMR) derivatives with an azacrown-ether ring fused with the 9-(2-carboxy)phenyl ring ..........................99

4.1. Synthesis via the initial formation of TMR derivatives. 100

4.2. Elaboration of a route to phthalic acid derivatives with an azacrown-ether fused to the aromatic ring . 
Chapter 3. Synthesis of SplAsH-based fluorescent labels for tetracysteine tags and their bioimaging tests.

1. Development of biarsenical probes ..........................................................................110

2. Synthesis of precursors for SplAsH-based fluorescent labels ........................ 118

3. Synthesis of reactive fluorescent labels.............................................................122

3.1. Synthesis of the water-soluble labels ................................................................ 122

3.2. Synthesis of the TMR derivative intended for the localization of the tagged

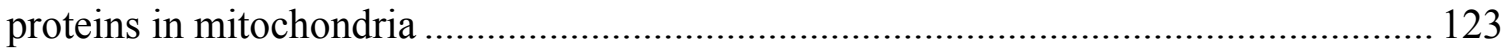

4. Spectroscopic control of the prepared biarsenical probes............................... 124

5. Bioimaging tests of the prepared biarsenical probes .......................................... 127

Experimental Part............................................................................................................. 135

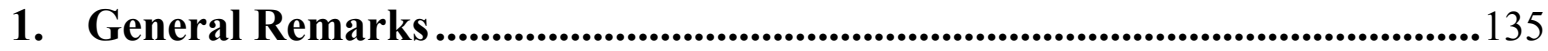

2. Preparation of Known Compounds ..............................................................136

3. Experimental procedures .......................................................................................... 138

Summary ................................................................................................................................. 165

References.................................................................................................................................... 169

Spectral Data.................................................................................................................................. 177 


\section{Abbreviations}

\begin{tabular}{|c|c|}
\hline $\mathrm{Ac}_{2} \mathrm{O}$ & acetic anhydride; \\
\hline $\mathrm{AcCl}$ & acetyl chloride; \\
\hline $\mathrm{AcOH}$ & acetic acid; \\
\hline \multirow{2}{*}{\multicolumn{2}{|c|}{$\begin{array}{l}\text { Amino acid } \\
\text { residues: }\end{array}$}} \\
\hline & \\
\hline $\mathrm{P}$ & proline; \\
\hline G & glycine; \\
\hline $\mathrm{S}$ & serine; \\
\hline $\mathrm{C}$ & cysteine; \\
\hline APTRA & 2-aminophenoxy- $N, N, N^{\prime}$-triacetic acid; \\
\hline BAPTA & 1,2- $\underline{\text { bis }}\left(o\right.$-aminophenoxy)ethane- $N, N, N^{\prime}, N^{\prime}$-tetraacetic acid; \\
\hline $\mathrm{BnCl}$ & benzyl chloride; \\
\hline BODIPY & 4,4-difluoro-4-bora-3a,4a-diaza-s-indacene; \\
\hline $\mathrm{BuCN}$ & valeronitrile; \\
\hline $\mathrm{C}_{10} \mathrm{H}_{8}$ & naphthalene; \\
\hline $\mathrm{dba}$ & dibenzylideneacetone; \\
\hline DBU & 1,8-diazabicyclo[5.4.0]undec-7-ene; \\
\hline DIPEA & $N, N$-diisopropylethylamine; \\
\hline DMAA & $N, N$-dimethylacetamide; \\
\hline DMAP & 4-( $N, N$-dimetylamino)pyridine; \\
\hline DME & 1,2-dimethoxyethane; \\
\hline DMF & $N, N$-dimethylformamide; \\
\hline DMSO & dimethylsulfoxide; \\
\hline EDC & 1-[3-(dimethylamino)propyl]-3-ethylcarbodiimide; \\
\hline EDTA & $N, N, N^{\prime}, N^{\prime}$-ethylenediamine tetraacetate; \\
\hline ESIPT & excited state intramolecular proton transfer; \\
\hline $\mathrm{Et}_{2} \mathrm{SO}_{4}$ & diethylsulfate; \\
\hline
\end{tabular}


EtOAc ethyl acetate;

FlAsH $\quad$ fluorescein arsenic hairpin binder;

FRET fluorescence resonance energy transfer;

GFP green fluorescent protein;

HMPTA hexamethylphosphortriamide;

HOMO highest occupied molecular orbital;

ICT intramolecular charge transfer;

$i \mathrm{PrOH} \quad$ 2-propanol,

ISC inter system crossing;

LUMO lowest unoccupied molecular orbital;

$\mathrm{MeCN} \quad$ acetonitrile;

$\mathrm{MeNH}_{2} \quad$ methylamine;

MES 2-mercaptoethanesulfonic acid;

MLCT metal-to-ligand charge transfer;

$\mathrm{MsCl}$ methanesulfonyl chloride;

n-BuOH 1-butanol;

NHS $\quad N$-hydroxysuccinimidyl;

PALM photoactivated localization microscopy;

PBS phosphate-buffered saline;

PCT photo charge transfer;

PET photo electron transfer;

PFA polyformaldehyde;

$p$ - $\mathrm{Tos} \mathrm{OH} \cdot \mathrm{H}_{2} \mathrm{O} \quad$ para-toluene sulfonic acid monohydrate;

Py pyridine;

SDS-PAGE dodecyl sulfate polyacrylamide gel electrophoresis;

SplAsH $\quad$ spirolactam arsenic hairpin binder; 
STED stimulated emission depletion;

TAPD tetraalkyl $p$-phenylenediamine;

$t$-BuOK $\quad$ potassium tert-butylate;

$t$-BuONa $\quad$ sodium tert-butylate;

TCEP tris(2-carboxyethyl)phosphine;

TFA trifluoroacetic acid;

THF tetrahydrofurane;

TMR tetramethyl rhodamine;

TMSCl trimethylsilyl chloride;

TMSI trimethylsilyl iodide;

$\mathrm{TrCl} \quad$ chlorotriphenylmethane;

Triton - X100 4-(1,1,3,3-tetramethylbutyl)phenyl-polyethylene glycol;

$\mathrm{TsCl} \quad$ para-toluene sulfonyl chloride;

TSTU N, N, $N^{\prime}, N^{\prime}$-tetramethyl- $O$-( $N$-succinimidyl)uronium tetrafluoroborate;

Z benzyloxycarbonyl. 


\section{Preface}

Cells and their organelles are the main objects of biological research. The problem of the low natural contrast of the microscopic cell images was noted long ago. Most of the cell compartments have weak colors, and they and other objects inside cells could not easily be distinguished. Hundreds of staining procedures have been developed since the middle of the $19^{\text {th }}$ century. ${ }^{[1]}$ Most of them are based on the local introduction of dyes into the cell components. Initial dyes were non-fluorescent and they selectively marked the required cell compartments by the interaction with the cell components without covalent binding. Nowadays, modern fluorescent labels are represented by the genetically encoded proteins, ${ }^{[2]}$ or adducts of the fluorescent dyes with small molecules (recognizable units) which react with genetically encoded tags. ${ }^{[3]}$ Within this great set of available labelling reagents, there are not only fluorescent compounds for imaging but also molecular probes which provide valuable information on the actually occuring events in cells, e. g. ion-channels opening or closing, protein polymerization or depolymerization, signal transduction, protein synthesis and so on. One kind of molecular probes - fluorescent calcium indicators - play a very important role in studying the calcium distribution and dynamics in various biological objects and processes.

The first part of this work deals with the design and synthesis of calcium indicators

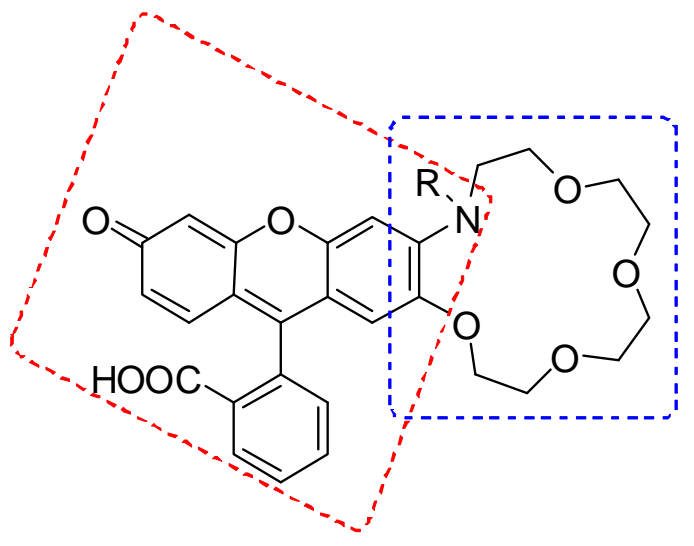

Figure 1. The first group of $\mathrm{Ca}^{2+}$ indicators prepared in this work (the red dashed line marks the fluorophore moiety, the blue dashed line - ionophore moiety), $\mathrm{R}=\mathrm{H}$, Et, $\mathrm{CH}_{2} \mathrm{COOH}$. (sensors). There are two kinds of the new calcium probes: genetically encoded calcium-sensitive proteins (most of them originate from aequorin ${ }^{[4]}$ ) and indicators, based on fluorescent organic dyes. Calcium-sensitive proteins have significant drawbacks: their molecular weight is about $27 \mathrm{kDa}$, and they may perturb the parent protein. Moreover, they have a low photostability and a limited range of "working" wavelengths. On the other hand, small organic dye molecules are more photostable (especially xanthenes), and their chemistry is well developed.

For reasons of its good spectroscopic properties, rhodol (3-amino-6-hydroxy-9phenylxanthene) was chosen as a fluorophore moiety for the first group of indicators (see Figure 1). The coordinative element of these sensors was realized in the form of an azacrown ether with a suitable cavity for calcium cations. Another key fragment of a new indicator is 
the carboxy group in the ortho-position of a phenyl ring attached to rhodol. It can provide a photoswitching ability of this dye (e. g. by creating a photosensitive spiroamide $^{[5]}$ or spirodiazoketone ${ }^{[6]}$ ) which may be an important requirement for PALM (photoactivated localization microscopy) method.

The first target group of indicators (Figure 1) was prepared in 13-15 steps. The spectroscopic properties of the new sensors have been established, and their applicability in the role of indicators for calcium was evaluated.

Another group of the target sensors is depicted in Figure 2. In them, the crown ether ring is fused with the phenyl group at C-9 of the xanthene

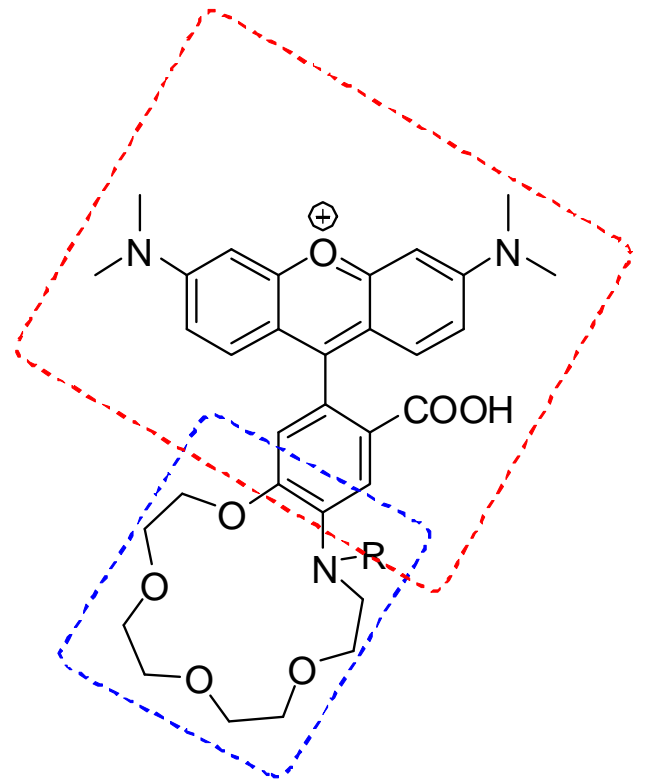

Figure 2. The second target group of sensors (the red dashed line marks the fluorophore moiety, the blue dashed line - ionophore moiety), $\mathrm{R}=\mathrm{Et}, \mathrm{CH}_{2} \mathrm{COOH}$. residue, and the chromophore has been changed to rhodamine. The tetramethylrhodamine backbone was envisaged in order to increase the membrane permeability of the fluorophore.

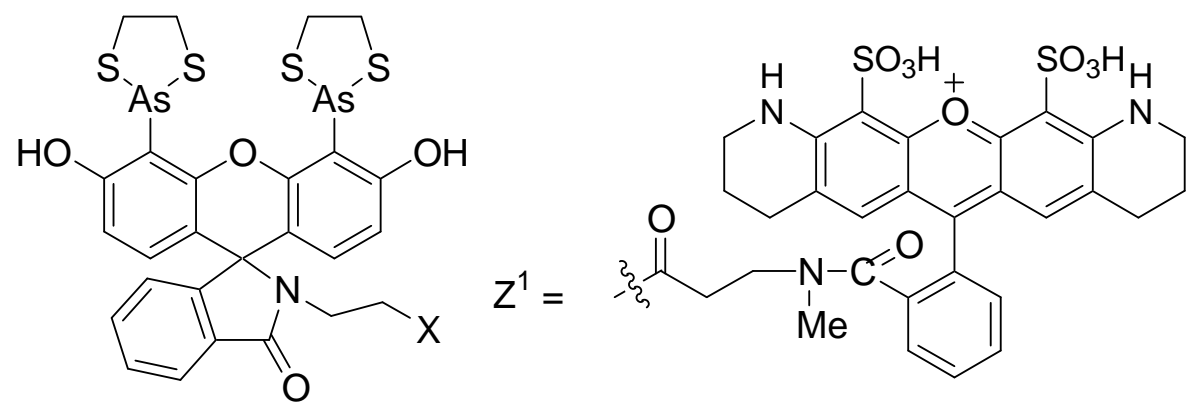

SplAsH: $X=\mathrm{NH}_{2}$;

SplAsH-RhS-OF: $X=\mathrm{NH}-\mathrm{Z}^{1}$;

SplAsH-RhS-CF: $X=\mathrm{NH}-Z^{2}$;

SpIAsH-TAMRA: $X=\mathrm{NH}-\mathrm{Z}^{3}$.

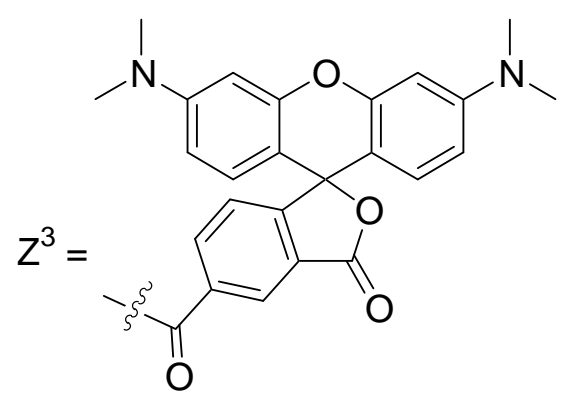<smiles>[Z]#CCCCN1C(=O)c2ccccc2C12c1cc3c(c(S(=O)(=O)O)c1Oc1c2cc2c(c1S(=O)(=O)O)NCCC2)NCCC3</smiles>

Figure 3. Adducts of SplAsH with the xanthene dyes, applied for the labeling. 
The second part of this work deals with an attempt of the selective labelling of peptides and proteins with fluorescent dyes. For this, three adducts of the so-called $\mathrm{SplAsH}^{[7]}$ with NHS esters of the "open" and "closed" isomers of Rhodamine $\mathrm{S}^{[8]}$ amides and NHS esters of TAMRA $^{[9]}$ were prepared (Figure 3 ). The binding properties of the compounds SplAsH-RhSOF, SplAsH-RhS-CF, SplAsH-TAMRA were studied by spectroscopic and biological tests. 


\section{Chapter 1. Fluorescent $\mathrm{Ca}^{2+}$ sensors for imaging calcium distributions in biomatter}

\section{Fluorescence and principles of fluorescence microscopy}

In the XVIth century, the Spanish physician Nicolas Monardes discovered the phenomenon of fluorescence, ${ }^{[10]}$ and since that time, there is an ever increasing interest in this process, the expression of which was coined by G. Stokes, ${ }^{[11]}$ (Scheme 1).

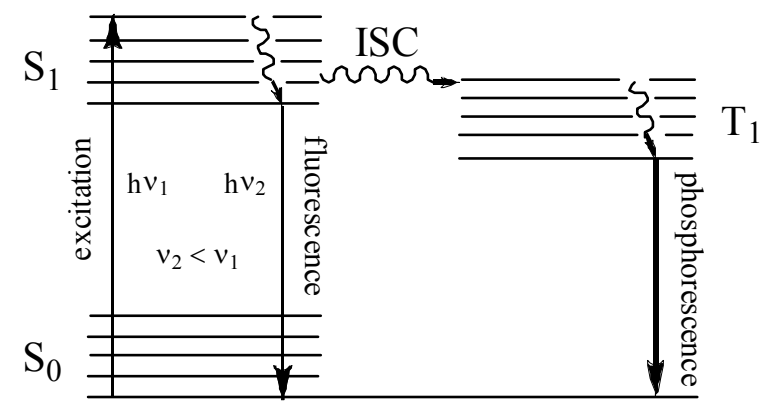

Scheme 1. Energy levels of a molecule and electronic transitions between them caused by the interaction with light (ISC - Inter System Crossing).

Being exposed to light, molecules may be excited from the ground electronic state $\left(\mathrm{S}_{0}\right)$ to the first excited state $\left(S_{1}\right)$. After that, the molecule can emit the photon of the longer wavelength and return to the ground state $\mathrm{S}_{0}$. This process is called fluorescence. However, the molecule can return to the ground state $\left(\mathrm{S}_{0}\right)$ not only directly, but also via a triplet state $\left(\mathrm{T}_{1}\right)$. These transitions $\left(\mathrm{S}_{1} \rightarrow \mathrm{T}_{1} \rightarrow \mathrm{S}_{0}\right.$ or $\mathrm{S}_{1} \rightarrow \mathrm{T}_{1} \rightarrow \mathrm{S}_{1} \rightarrow \mathrm{S}_{0}$ ) are called phosphorescence or delayed fluorescence. The processes without light emission, for example, internal conversion or energy transfer may also occur. Other possible processes in the excited state are conformational change, proton transfer, electron transfer, chemical reaction or excimer (exciplex) formation. From these abundant post-excitation changes, only three radiative transitions (fluorescence, phosphorescence and delayed fluorescence) may give an immediate physical response (in the form of light) on the presence of other molecules in the medium, because the energy of all electronic states $\left(S_{n}, T_{n}\right)$ directly depend on the surrounding of the fluorescent probe. The spectral analysis of the emitted light may provide data on the environment of a fluorescent probe. The fluorescence is the most straightforward process that can be used as an effective analytical tool. Typical parameters of the medium, which can affect the fluorescence, are the polarity, the presence of ions and quenchers, hydrogen bonds, $\mathrm{pH}$, pressure, viscosity, temperature and electrical potential. 
As it concerns the detection of "foreign" molecules in the medium, the fluorescence played a very important role in the development of microscopy. The light generates the picture on the retina (or a detector matrix). By that, there is no difference, whether reflected or emitted light is used for imaging. Reflected light is used in conventional optical microscopy, and emitted light - in fluorescence microscopy, which started to emerge since the beginning of the 20th century. ${ }^{[12]}$ As early as 1930 the first fluorescence microscope was produced by Carl Zeiss.

Microscopy helps one to observe very small objects. But what are the limits of this method? Such kind of questions arose more than one century ago. The diffraction limit for optical microscopy was found by Ernst Abbe. ${ }^{[13]}$ He established that an optical microscope cannot resolve objects that are separated by the distance which is less than one half of the wavelength of the light used. Abbe's law can be mathematically expressed as follows: $\boldsymbol{d}=\lambda /$ $(2 n \sin \alpha)(1)$, in which $\boldsymbol{d}$ is a minimal distance between the two resolved objects in $\mathrm{nm}, \lambda$ the wavelength of the used light, $n$ the refractive index of the medium, $\alpha$ the aperture angle of the lens. The diffraction barrier was considered to be an absolute limitation and could not be overcome for more than a century. Only in 1994 did, the publication of S. W. Hell and J. Wichmann ${ }^{[14]}$ reveal the possibility to break the diffraction limit. The general concept of the high-resolved imaging was formulated by S. Hell in 2004. ${ }^{[15]}$ This concept is applicable to the fluorescence microscopy, where the objects are labelled with fluorescent markers. Thus, the light emitted from these markers forms an image. The optical resolution can be improved by spatial contraction of the imaged fluorescent area applying a special doughnut-shaped laser beam that depletes the population of the excited electronic state. This nanoscopic method is called stimulated emission depletion (STED). ${ }^{[16]}$ The quantitative description of the STED method is expressed by the following equation.

$$
d=\frac{\lambda}{2 n \sin \alpha \sqrt{1+I_{\max } / I_{s}}}
$$

Here, $I_{\mathrm{s}}$ stands for saturation intensity (the light intensity which makes the populations of the ground and the (first) excited states to be equal), $I_{\max }$ for the applied intensity of the STED beam, for the definitions of $\lambda, \mathrm{n}$ and $\alpha$, see eq. 1 . 


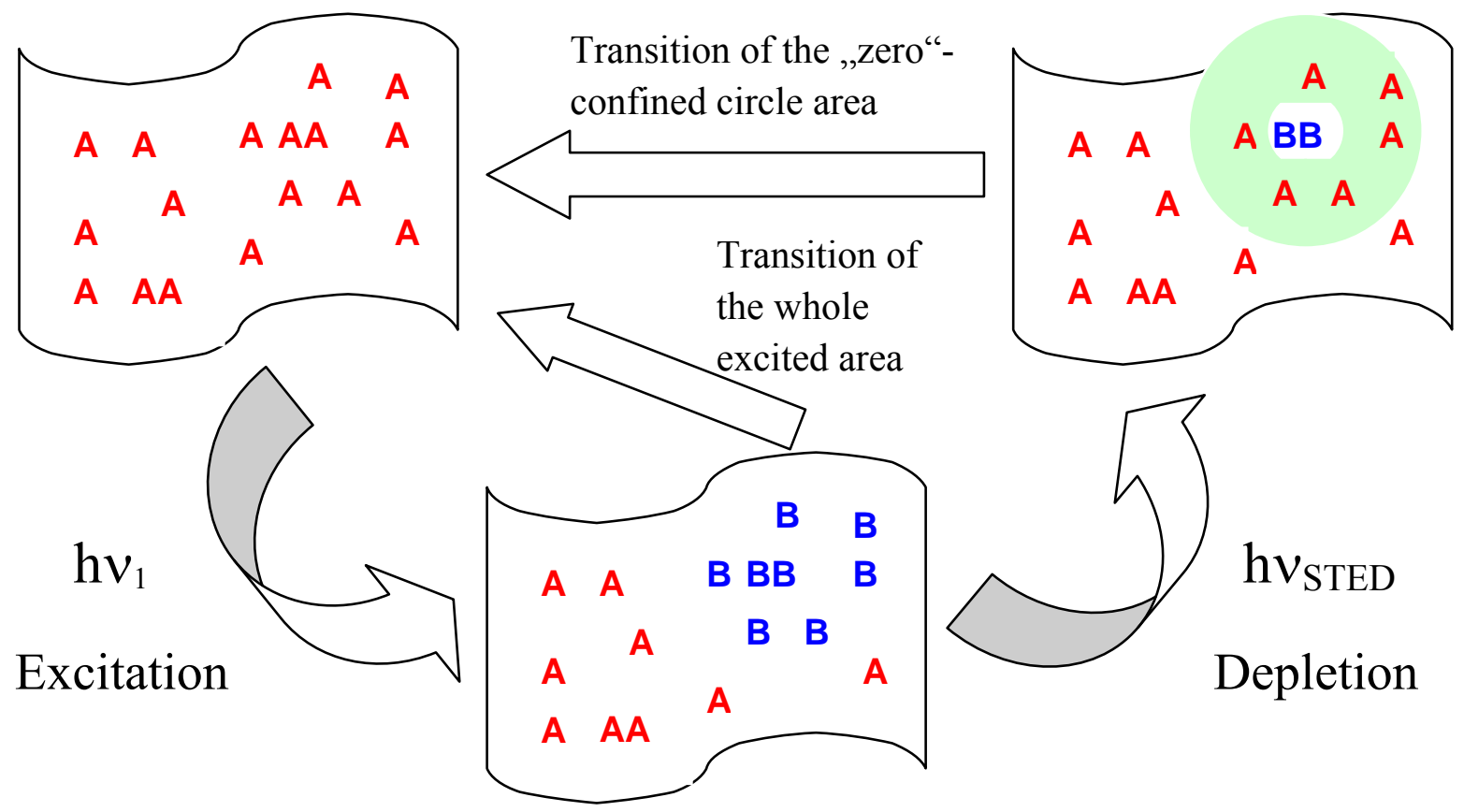

Scheme 2. The principle of STED microscopy. Red-marked "A"-molecules are the molecules of a fluorescent marker in the ground state $\mathrm{S}_{0}$, blue-marked "B"-molecules are the molecules of a fluorescent marker in the first excited state $S_{1} ; v_{1}$ is the frequency of excitation light, $v_{\text {STED }}$ the frequency of the doughnut-shaped STED beam; the green doughnut represents the area, where the excited molecules were depleted by the STED beam.

To understand, how the fluorescent area in the STED microscopy can be reduced, one should consider the two states of a fluorescent marker: the initial "dark" state A, and the "bright" (excited) state B. The excitation by the laser beam applied to the small area of the fluorescent markers converts them from the state $\mathbf{A}$ into the state $\mathbf{B}$. If the molecules in this area are allowed to return back from the excited state $\mathbf{B}$ into the state $\mathbf{A}$ spontaneously, then a blurred diffraction-limited image on the detector will be obtained. On the other hand, if the Bstate zone is immediately illuminated with a second, doughnut-shaped laser beam, all B-state molecules are converted into the initial state $\mathbf{A}$, with the exception of a small area in the center of the B-state zone. Then the molecules from this area will emit light which will be recorded by the detector. The size of this fluorescent area is given by equation (2). Thus, in principle, the attained resolution may be increased to be "infinite"; however, at huge of light intensities the fluorescent markers bleach.

There is another nanoscopic method called "PALM" (PhotoActivation Localization Microscopy). ${ }^{[17]}$ In the PALM method, also two states of the marker are used. State $\mathbf{A}$ is non- 
fluorescent, and state $\mathbf{B}$ is fluorescent. With light of low power, the single marker molecules are converted from the non-fluorescent state $\mathbf{A}$ into the fluorescent state $\mathbf{B}$. In order to determine the positions of single molecules, it is necessary to collect emitted photons. The positions of single molecules can be calculated with a localization precision of $x=F W H M / \sqrt{N}$, where FWHM (full width at half-maximum) is a device constant (typically $\approx 300 \mathrm{~nm}$ ), and $N$ the number of collected photons.

The general concept of optical nanoscopy may be formulated as follows. The objects, which can (reversibly) be photoswitched between the non-fluorescent state $\mathbf{A}$ and the fluorescent state B (see Figure 4), may be optically resolved by application of the finite intensity of light, if they are situated at a distance greater than $\boldsymbol{d}$ (Equation 2) or $x$ (see above).

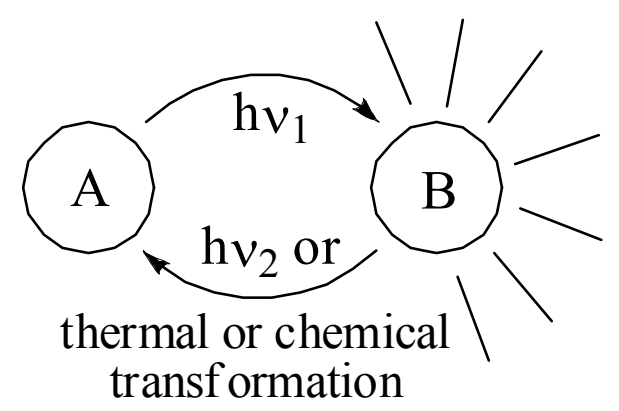

Figure 4. Light-induced transition between the "dark" state A and the "bright" state B.

Up to now, only a very limited number of reports deal with the applications of nanoscopic methods in metal sensing. ${ }^{[18]}$ As it was already mentioned, it is possible to determine the concentration of many metal ions by measuring the fluorescence signal. Some cations emit light themselves (for example, lanthanides and actinides), and the non-fluorescent metal ions may be detected with the help of organic indicators. For example, the presence and the concentration of calcium cations may be detected by various fluorescent sensors. Calcium plays an important role as a second messenger in various biological processes, such as cell growth, transcriptions (DNA to mRNA), metabolism, exocytosis, muscle contraction, apoptosis and so on. ${ }^{[19]}$ Therefore, it became very important to find a method for the evaluation of calcium concentrations and the distribution of calcium in living cells. In the 1970s it was only possible to localize calcium ions in cells either by precipitating them with oxalate and studying the precipitated granules by electron microscopy ${ }^{[20]}$ or by X-ray analysis of samples marked with ${ }^{45} \mathrm{Ca} .{ }^{[21]}$ Nowadays, various nanoscopic techniques are capable, in principle, not only of registering the distribution of calcium concentration within the cell, but also, when using STED microscopy, even the dynamics of calcium ions distribution inside the 
cells can be revealed. For example, the use of conventional calcium dyes enabled to acquire the images of synaptic calcium microdomains of cochlear inner hair cells. ${ }^{[18]}$

A wide range of fluorescent calcium sensors is available nowadays, but only some of them are applicable in far-field fluorescent microscopy. Therefore, we decided to design and to synthesize some new calcium sensors, which meet the following requirements:

1) possess excitation and emission maxima in the region above $450 \mathrm{~nm}$;

2) have high "brightness" of a dye (brightness is the product of the fluorescence quantum yield and an extinction coefficient);

3) can be masked (caged) or made switchable (due to the presence of a free carboxyl group on the phenyl ring in the ortho-position relative to the xanthane fragment);

4) have high photostability;

5) possess high selectivity to calcium ions (selectivity is the ratio of dissociation constants of the complexes with two different cations; see equations for $K_{\mathrm{D}}$ below);

6) are able to penetrate through cell membrane.

The equation for the stability and dissociation constants $\left(K_{\mathrm{S}}\right.$ and $\left.K_{\mathrm{D}}\right)$ of a metal complex (IM) formed by the metal ion (M) and an indicator (I) is given in Figure 5. In many cases the highest possible sensitivity is required.

$$
\mathrm{I}+\mathrm{M} \rightleftarrows \mathrm{IM} \quad K_{\mathrm{S}}=\frac{1}{K_{\mathrm{D}}}=\frac{[\mathrm{IM}]}{[\mathrm{I}] *[\mathrm{M}]}
$$

Figure 5. The dissociation reaction and equation for the dissociation constant $K_{\mathrm{D}}$ and stability constant $K_{\mathrm{s}}$ of the complex IM, in which I stands for indicator molecule, M for metal ion.

However, sometimes only a moderate sensitivity to calcium is required. For example, a low affinity $^{[22]}$ to calcium with $K_{\mathrm{D}}>90 \mu \mathrm{M}$ is necessary in some microscopic applications. Moreover, the ratiometric properties (see section 2.1) of calcium indicators are required in most applications. Ratiometric probes provide a shift of the extinction and emission maxima of the fluorescent sensor upon formation of a complex with an analyte. This effect may also be very helpful in optical nanoscopy, e. g. in the single-molecule switching microscopy (in spite of its low temporal resolution). ${ }^{[23]}$ In this case, the superresolution techniques may provide "calcium mapping" - the spatial distribution of calcium concentration inside a cell. 


\section{Fluorescent indicators for calcium cations}

A typical calcium indicator consists of a fluorophore and an ionophore residue. The fluorophore moiety is responsible for the modulation of the fluorescence signal upon coordination with a calcium cation. The ionophore part must be selective enough to bind calcium ions from the environment. All fluorescence sensors may be classified according to the photophysical properties of their fluorescent parts or according to the structure of their calcium binding sites. The second classification will be pursued here with a short description of the photophysical properties of the corresponding sensors. Before that, the main photophysical principles of ion sensing by fluorescent indicators are described.

\subsection{Main photophysical principles of ion sensing by fluorescent indicators and determination of calcium concentrations from the spectroscopic data}

Two photophysical principles are applicable for calcium sensing: photoinduced electron transfer (PET) and photoinduced charge transfer (PCT). In the first case (PET), a donor group as part of the complexant is connected with the conjugated aromatic system of a fluorescent dye through a bridge. The donor group quenches the fluorescence of a dye due to the electron transfer from its HOMO (highest occupied molecular orbital) to the „half-filled” HOMO of the excited fluorophore (see Scheme 3), which has a lower energy. In the absence of calcium, the HOMO is mostly localized on the lone pair of a heteroatom. The sensor molecule becomes highly fluorescent upon calcium coordination, because the calcium ion acting as a Lewis acid binds by way of the lone electron pairs of the donor group. Therefore, the energy of the HOMO of the donor group in the complex decreases and the electron-transfer becomes impossible.

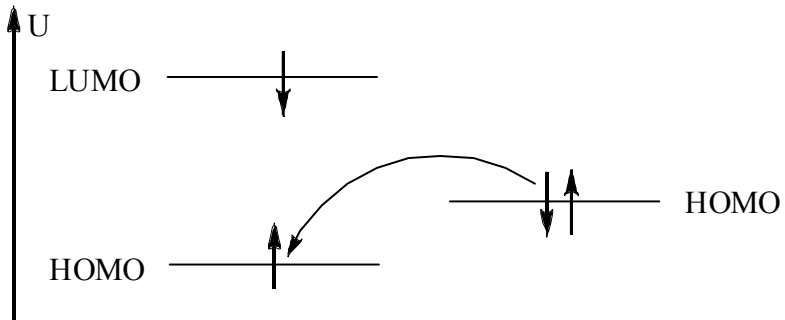

Excited Fluorophore

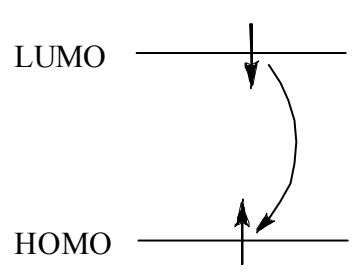

Excited Fluorophore

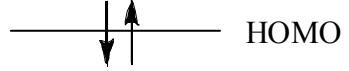

Calcium-bound receptor

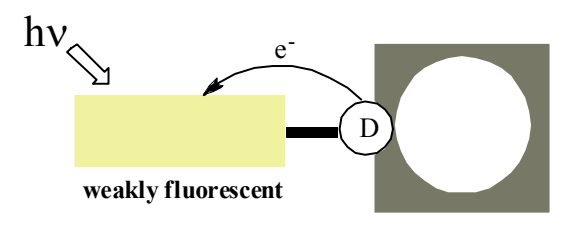

D - Donor

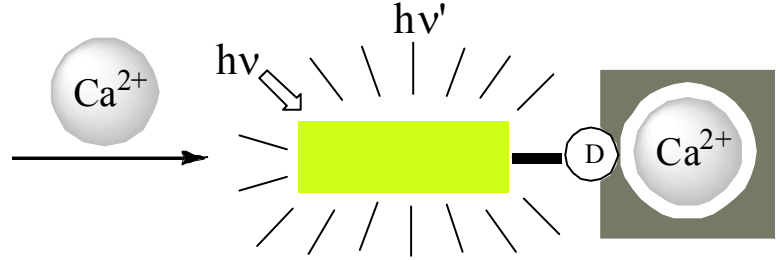

strongly fluorescent 
Scheme 3. Functional principles of PET-based calcium sensing. ${ }^{[10]}$

In the case of PET sensors, the intensity increase of the fluorescence signal can be high enough to evaluate the calcium ion concentration. For this, it is necessary to know the fluorescence intensity of the indicator solution in the absence of calcium ( $I_{\text {free }}^{f l}$ ), the fluorescence intensity of the same solution "saturated" with calcium $\left(I_{\text {sat }}^{f l}\right)$, the value of the dissociation constant for the given calcium indicator $K_{\mathrm{D}}$, and the fluorescence intensity of the solution with unknown $\left[\mathrm{Ca}^{2+}\right]\left(I_{x}^{f l}\right)$. The calcium ion concentration can then be calculated according to equation (3).

$$
\left[C a^{2+}\right]=K_{D}\left(\frac{I_{x}^{f l}-I_{\text {free }}^{f l}}{I_{\text {sat }}^{f l}-I_{x}^{f l}}\right)
$$

However, in order to evaluate the absolute value of a local calcium concentration, all values $I_{\text {free }}^{f l}, I_{\text {sat }}^{f l}$ and $I_{x}^{f l}$ have to be determined at the same total concentration of a dye, optical path length and instrumental sensitivity. For measurements inside cells, the latter conditions can not always be guaranteed.

The second principle - PCT - is applied in sensors that contain an ionophoric part integrated into a fluorescent dye, in which a calcium-sensitive electron-donor (or even electron-acceptor) group is conjugated with another electron-acceptor or (donor) group via an aromatic system. Upon excitation, a full charge transfer of the donor group to the acceptor group occurs. The excited state becomes more polar than the ground state. Therefore, interaction of a calcium cation with an acceptor group causes an decrease of energy of the excited and ground states (see Scheme 4a). These phenomena are more pronounced for the excited states than for the ground states. As a consequence, a red shift in the absorption and fluorescence spectra of a complex is observed (compared with the free ligand); and often the molar absorption coefficient of a complex increases (compared with the $\varepsilon$-value for the free ligand). Conversely, coordination of a cation with the donor moiety of the sensor causes destabilization of $S_{1}$ state to a higher degree than of the $S_{0}$ state (see Scheme $4 b$ ), which leads to a blue shift of both spectra of the complex (compared with the free ligand) and a decrease in the molar absorption coefficient (compared with the $\varepsilon$-value for the free ligand). Also, the changes of other fluorescence parameters like quantum yields and lifetimes of the excited states can be observed. 


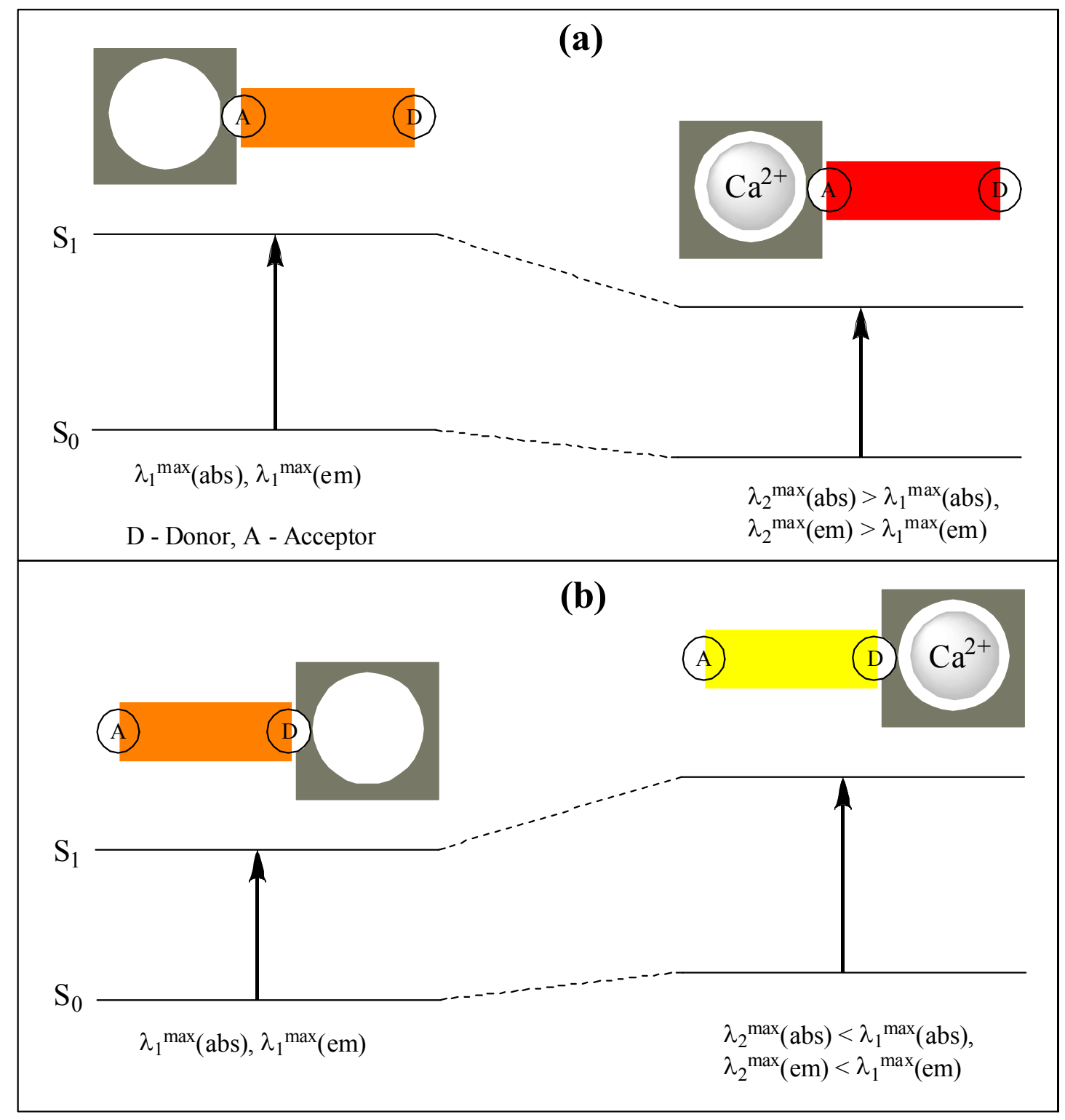

Scheme 4. Characteristic changes in the energies of the ground and excited states and the transitions $\left(\mathrm{S}_{0} \rightarrow \mathrm{S}_{1}\right)$ responsible for the spectral bands of the PCT-based calcium ion sensors: (a) the case, when the coordination moiety (depicted as a grey area) pulls the calcium ion towards the acceptor group; (b) the case, when the coordination moiety (depicted as a grey area) pulls the calcium ion towards the donor group. ${ }^{[10]}$

In order to be able to calculate calcium concentrations in the case of PCT sensors, it is necessary to have compounds with large red or blue shifts of the emission or absorption bands of a complex (compared to these parameters for the free ligand). To estimate the concentration in the ratiometric mode, it is necessary to know the ratios of the fluorescence intensities $(R)$ (not absolute intensities of the fluorescence band, as in the fluorescent mode) 
for the complex and the free ligand. Moreover, it is necessary to know the ratio of the fluorescence intensities of the indicator solution at two different wavelengths $-R_{\min }$; the ratio of fluorescence intensities of the same indicator solution, but "saturated" with calcium at the same wavelengths $-R_{\max }$; the dissociation constant for the complex with calcium $\left(K_{\mathrm{D}}\right)$, the ratio of the fluorescence intensities of the working solution $\left(R_{\mathrm{x}}\right)$ (at the two different wavelengths), and the ratio of the fluorescence intensities of the free and bound forms of the indicator at the same (one) wavelength $I_{\text {free }}^{f l}\left(\lambda_{2}\right) / I_{\text {Ca-bound }}^{f l}\left(\lambda_{2}\right)$. With these parameters it is possible to calculate the calcium concentration according to the equations (4).

$$
\left[C a^{2+}\right]=K_{D}\left(\frac{R_{x}-R_{\min }}{R_{\max }-R_{x}}\right) \frac{I_{\text {free }}^{f l}\left(\lambda_{2}\right)}{I_{\text {Ca-bound }}^{f l}\left(\lambda_{2}\right)} \quad R_{x}=\frac{I_{x}^{f l}\left(\lambda_{1}\right)}{I_{x}^{f l}\left(\lambda_{2}\right)} \quad R_{\min }=\frac{I_{\text {free }}^{f l}\left(\lambda_{1}\right)}{I_{\text {free }}^{f l}\left(\lambda_{2}\right)} \quad R_{\max }=\frac{I_{C a-\text { bound }}^{f l}\left(\lambda_{1}\right)}{I_{\text {Ca-bound }}^{f l}\left(\lambda_{2}\right)}
$$

In spite of the more complex calculations, the ratiometric mode has important advantages: the dye content and the instrumental sensitivity may be changed from one experiment to another.

\subsection{Diverse coordination moieties in fluorescent $\mathrm{Ca}^{2+}$ indicators}

The stable form of calcium in aqueous solutions is a dication with a linear charge density of $2.02 \mathrm{q} \AA^{-1}$ and an ionic radius of $1.98 \AA$. The most stable coordination numbers are $6-9 .{ }^{[10]}$ The following four ligands (Figure 6) provide the most stable $\mathrm{Ca}^{2+}$ complexes:

1) chelators (amino acids or amino ethers with carboxy groups);

2) podands (oligoethers or amino oligoethers with open chains);

3) coronands (usually crown ethers or amino crown ethers with 5 or 6 heteroatoms);

4) calixarenes (cup-shaped ligands with structures that fix the spatial positions of oxygen atoms). 


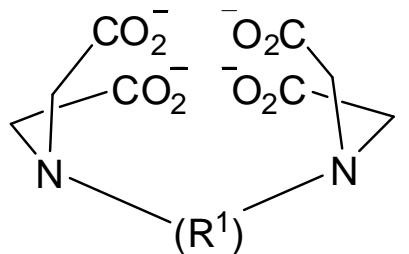

Chelators

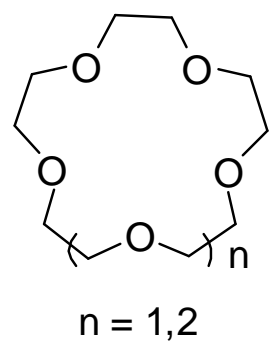

Coronands<smiles>[R]OCCOCCOCCO[R]</smiles>

Podands

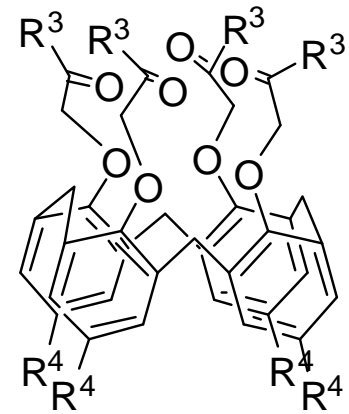

Calixarenes

Figure 6. Typical "recognizing" fragments used in fluorescent calcium sensors. If $\mathrm{R}^{1}=$ $\mathrm{CH}_{2} \mathrm{CH}_{2}$, then the chelator is EDTA - the known analytically useful complexant. If $\mathrm{R}^{1}$ contains an aromatic system, then the chelator gets some sensoric properties. $\mathrm{R}^{2}$ is usually an aromatic or heteroaromatic substituent. $\mathrm{R}^{3}$ is an aromatic substituent (naphthyl, anthracenyl). $\mathrm{R}^{4}$ is a bulky group, e. g. tert-butyl, neopentyl which is required for the orientation of the coordinating oxygen atoms that provides calcium complexation.

\subsection{Early steps in the creation of highly sensitive fluorescent calcium indicators for biological purposes}

Progress in analytical chemistry stimulated the development of fluorescent calcium indicators. Complexometry represents an important analytical tool, because it allows the determination of the calcium concentration of these dications by titration. The precise determination of the end point of titration provides accurate values of the dication concentration, if an indicator with a sharp visible transition exists. For example, Calcein (1) provides such kind of a sharp transition from a non-fluorescent brown form to a fluorescent yellow-green form at $\mathrm{pH}=12$ (Figure 7). ${ }^{[24]}$ Therefore, this indicator was superior to other non-fluorescent indicators. 


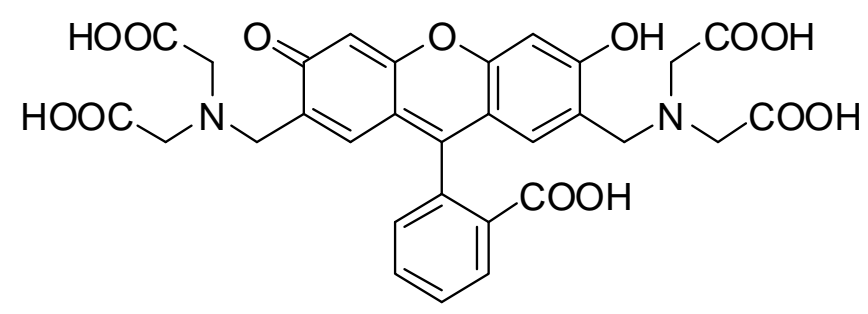

1

Figure 7. The analytical indicator Calcein for calcium dications. ${ }^{[24]}$

Compound 1 was synthesized from fluorescein, formaldehyde and imino diacetic acid employing a Mannich reaction. ${ }^{[2]}$ In spite of the fact that the coordination number of compound 1 is 5 (which is less than the optimal value 6), the threshold level for calcium was found to be low, and therefore, this compound was successfully used in complexometry.

Four years later, the indicator $\mathbf{2 b}$ was synthesized in a similar fashion (Figure 8). For this the fluorescent 4-methylumbelliferone $\mathbf{2 a}$ was used as a core structure. Due to its brilliant blue fluorescence, this compound was called Calcein Blue. ${ }^{[25]}$ In the absence of calcium, aqueous solutions of this substance are intensively fluorescent only at $\mathrm{pH} \leq 11$, but even at $\mathrm{pH}=12$, the solutions become fluorescent again, if a calcium salt is added. Unfortunately, in the original publication the synthesis was not reported, but some spectral data were given: the maxima of absorption $\left(\lambda^{\max }(\right.$ acidic solution $)=330 \mathrm{~nm}, \lambda^{\max }($ basic solution $\left.)=370 \mathrm{~nm}\right)$ and fluorescence $\left(\lambda_{f l}^{\max }=445 \mathrm{~nm}\right)$.<smiles></smiles>

2a: $R^{1}=R^{3}=R^{4}=H, R^{2}=M e ;$

2b: $\mathrm{R}^{1}=\mathrm{R}^{3}=\mathrm{H}, \mathrm{R}^{2}=\mathrm{Me}, \mathrm{R}^{4}=\mathrm{CH}_{2} \mathrm{~N}\left(\mathrm{CH}_{2} \mathrm{COOH}\right)_{2}$;

2c: $\mathrm{R}^{1}=\mathrm{COOEt}, \mathrm{R}^{2}=\mathrm{H}, \mathrm{R}^{3}=\mathrm{Me}, \mathrm{R}^{4}=\mathrm{CH}_{2} \mathrm{~N}\left(\mathrm{CH}_{2} \mathrm{COOH}\right)_{2}$;

2d: $\mathrm{R}^{1}=\mathrm{Ph}, \mathrm{R}^{2}=\mathrm{H}, \mathrm{R}^{3}=\mathrm{Me}, \mathrm{R}^{4}=\mathrm{CH}_{2} \mathrm{~N}\left(\mathrm{CH}_{2} \mathrm{COOH}\right)_{2}$;

2e: $\mathrm{R}^{1}=\mathrm{R}^{3}=\mathrm{R}^{2}=\mathrm{R}^{4}=\mathrm{H}$;

2f: $\mathrm{R}^{1}=\mathrm{R}^{2}=\mathrm{R}^{3}=\mathrm{H}, \mathrm{R}^{4}=\mathrm{CH}_{2} \mathrm{~N}\left(\mathrm{CH}_{2} \mathrm{COOH}\right)_{2}$.

Figure 8. Coumarin-based analytical indicators for calcium. ${ }^{[25,26,28]}$

The properties of the coumarin derivatives $\mathbf{2 c}, \mathbf{2 d}$ were reported by Huitink ${ }^{[26]}$ in greater details than the of Calcein Blue. The working principles of indicators $\mathbf{2} \mathbf{c}$ and $\mathbf{2 d}$ are the same as for compound $\mathbf{2 b}$. However, the working $\mathrm{pH}$ value for compounds $\mathbf{2} \mathbf{c}-\mathbf{d}$, was found to be 13. Excitation and emission maxima of the novel indicators $\mathbf{2 c - d}$ are red-shifted in 
comparison with these of Calcein Blue, due to the electron-withdrawing substituents on the coumarin core $\left[2 \mathbf{c}: \lambda^{\max }(\right.$ acidic solution $)=360 \mathrm{~nm}, \lambda^{\max }($ basic solution $)=409 \mathrm{~nm}, \lambda_{f l}^{\max }=447$ $\mathrm{nm}$ (the same for acidic and basic medium); $\mathbf{2 d}: \lambda^{\max }\left(\right.$ acidic soln.) $=348 \mathrm{~nm}, \lambda^{\max }$ (basic soln.) $=392 \mathrm{~nm}, \lambda_{f l}^{\max }=475 \mathrm{~nm}$ (the same for acidic and basic medium)]. Along with precise synthetic procedures, this report ${ }^{[26]}$ describes also the $\mathrm{pH}$ dependence of the fluorescent signal, which has a maximum at $\mathrm{pH}=8-9$. This maximum is related to the deprotonation of the ammonium residue. In modern terms, the deprotonation of the ammonium moiety quenches the fluorescence due to a PET effect. At higher $\mathrm{pH}$ values, the neutral amino group with its lone pair can transfer an electron to a fluorophore. At lower $\mathrm{pH}$ values $(<8-9)$, the decrease in the fluorescence intensity could be explained by the contribution of a nonradiative process in the energy dissipation (e. g. post-excitational dissociation of phenol group in 7-hydroxycoumarins. ${ }^{[27]}$ The fluorescence quantum yields of compounds $\mathbf{2 c}$ and $\mathbf{2 d}$ were 4 and 2, respectively, times higher than that of Calcein Blue. Both compounds $\mathbf{2 c}$, $\mathbf{d}$ were more stable in basic solutions than Calcein Blue.

Besides all values dissociation constants and other physical data for Calcein Blue in another report by Huitink and Diehl, ${ }^{[28]}$ the new indicator $\mathbf{2 f}$ was also described. It is based on the structure of umbelliferone (2e) and also turned out to be sensitive to calcium. Its spectroscopic properties are similar to those of Calcein Blue $\left[\lambda^{\max }\right.$ (acidic soln.) $=325 \mathrm{~nm}$, $\lambda^{\max }$ (basic soln.) $=367 \mathrm{~nm}, \lambda_{f l}^{\max }=455 \mathrm{~nm}$ (the same for acidic and basic medium)]. Unfortunately, compound $\mathbf{2} \mathbf{f}$ decomposes in basic solutions even faster than Calcein Blue.

With Calcein analogues, only high concentrations of calcium in aqueous solutions may be determined (in the range of ten millimolar and higher). Moreover, they require high $\mathrm{pH}$ values (12-13) which are far from physiological conditions in living cells. A breakthrough in the design of biologically relevant calcium sensors was realized after the report of Michaylova et al. appeared. ${ }^{[29]}$ In this work, the well-known metal-sensitive indicator Arsenazo III $^{[30]}$ was applied for the determination of micromolar $\mathrm{Ca}^{2+}$ concentrations by measuring the absorption at $600 \mathrm{~nm}$. The indicator Arsenazo III displayed an increase in the absorbtion maxima at 600 and $650 \mathrm{~nm}$ upon calcium complexation, within a linear interval of $0.030-1.50 \mathrm{mg} / \mathrm{L}$ of $\mathrm{Ca}^{2+}$. 


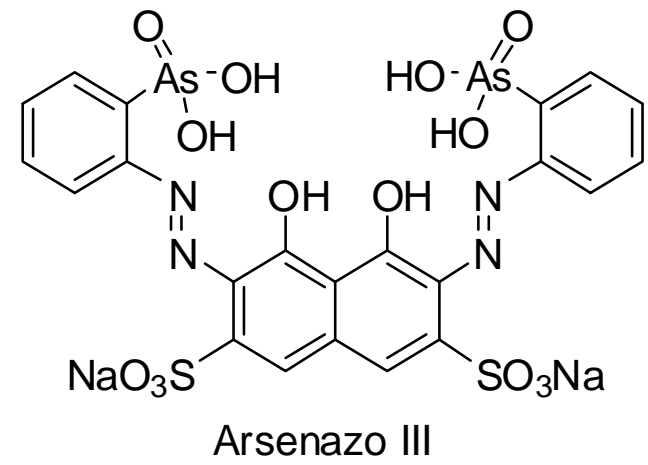

Figure 9. Spectrophotometrical metalloindicator. ${ }^{[30]}$

It was demonstrated that Arsenazo III is very sensitive to low concentrations of $\mathrm{Ca}^{2+}$ at physiological $\mathrm{pH}$ values (7.0-7.4). These results as well as an other advantage of Arsenazo III like its low molecular mass (compared to the calcium-sensitive protein aequorine), were used by Brown et al. in the study of the calcium activity in squid giant axons. ${ }^{[31]}$ They used the organic calcium sensor in living cells for the first time. Brown et al. studied small influxes of calcium, originating from changing the potential of the cell membrane.

\subsection{BAPTA and its derivatives}

In his pioneering work, Tsien realized the first rational design and synthesis of calcium indicators for biological applications. ${ }^{[32]}$

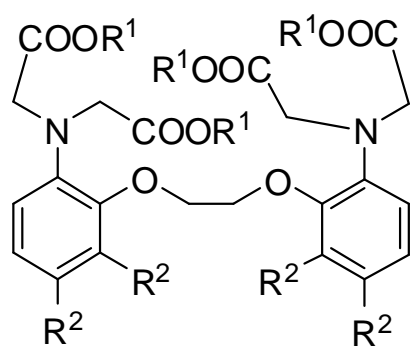

3a: $B A P T A: R^{1}=R^{2}=H$;

3b: TF-BAPTA: $\mathrm{R}^{1}=\mathrm{H}, \mathrm{R}^{2}=\mathrm{F}$.

Figure 10. BAPTA and its fluorinated analogue. ${ }^{[32,39 a-b]}$

All of them are based on the simple BAPTA (1,2-Bis(o-AminoPhenoxy)ethane$N, N, N^{\prime}, N^{\prime}$-Tetraacetic Acid) core. Compound 3a, the simplest indicator in this family, possesses two maxima in the absorption spectrum $(254$ and $287 \mathrm{~nm})$ and one maximum in the fluorescence spectrum (at $363 \mathrm{~nm}$ ). Calcium complexation shifts both absorption maxima to the blue region (new positions: 203 and $274 \mathrm{~nm}$ ) and decreases the intensity of the fluorescence maximum by a factor of 2.8. The parent compound $\mathbf{3 a}$ itself is useless for any 
application in biological studies, because it absorbs in the UV region. The UV light damages the cellular components containing the UV-sensitive amino and nucleic acids. The core of the BAPTA structure, however, may be modified. Tsien introduced a methyl group into the "right-hand" ring, and that increased the affinity of the derivate for calcium and its stability towards oxidation. Then the ether moiety was replaced with a pyridine ring, and that caused the required bathochromic shift of the absorption and emission bands from the 254 to $350 \mathrm{~nm}$ and from 363 to $520 \mathrm{~nm}$, respectively. The third modification was to introduce a methoxy group into the $m$-position relative to the amino diacetoxy fragment, and that improved the fluorescence quantum yield from 1.2 to $2.9 \%$. The resulting compound was called Quin-2 (see Figure 11). It possesses high calcium selectivity in the presence of $\mathrm{Mg}^{2+}$ and forms a $1: 1$ complex with $\mathrm{Ca}^{2+}$ (in comparison to the $2: 1$ ratio of Arsenazo III $-\mathrm{Ca}^{2+}$ ). ${ }^{[33]}$ This feature made Quin-2 quite popular, because it simplified the measurements.

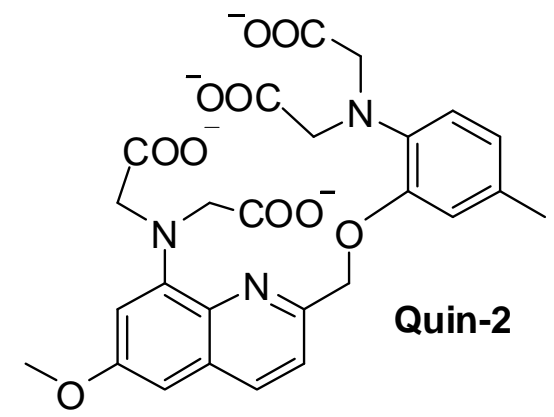

Figure 11. Calcium indicator Quin-2.

A further important step was made one year later, when Tsien invented a delivery method for the sensors into cells, without damaging the cell membrane.$^{[34]} \mathrm{He}$ transformed the initial compound, which could not pass the membrane, into the corresponding highly acetoxymethyl ester (Figure 10, $\mathrm{R}^{1}=\mathrm{CH}_{2} \mathrm{OAc}$ ), which penetrates the cell membrane easily. In the cytoplasm, enzymes (esterases) convert this ester into the initial water-soluble acid form.

After numerous practical tests, it was found that the photophysical properties of Quin-2 are not fully satisfactory for living cells. Thus, a new series of BAPTA derivatives was created. ${ }^{[35]}$ The most promising among them were the so-called Indo-1 and Fura-2, (Figure 12 and Table 1). 


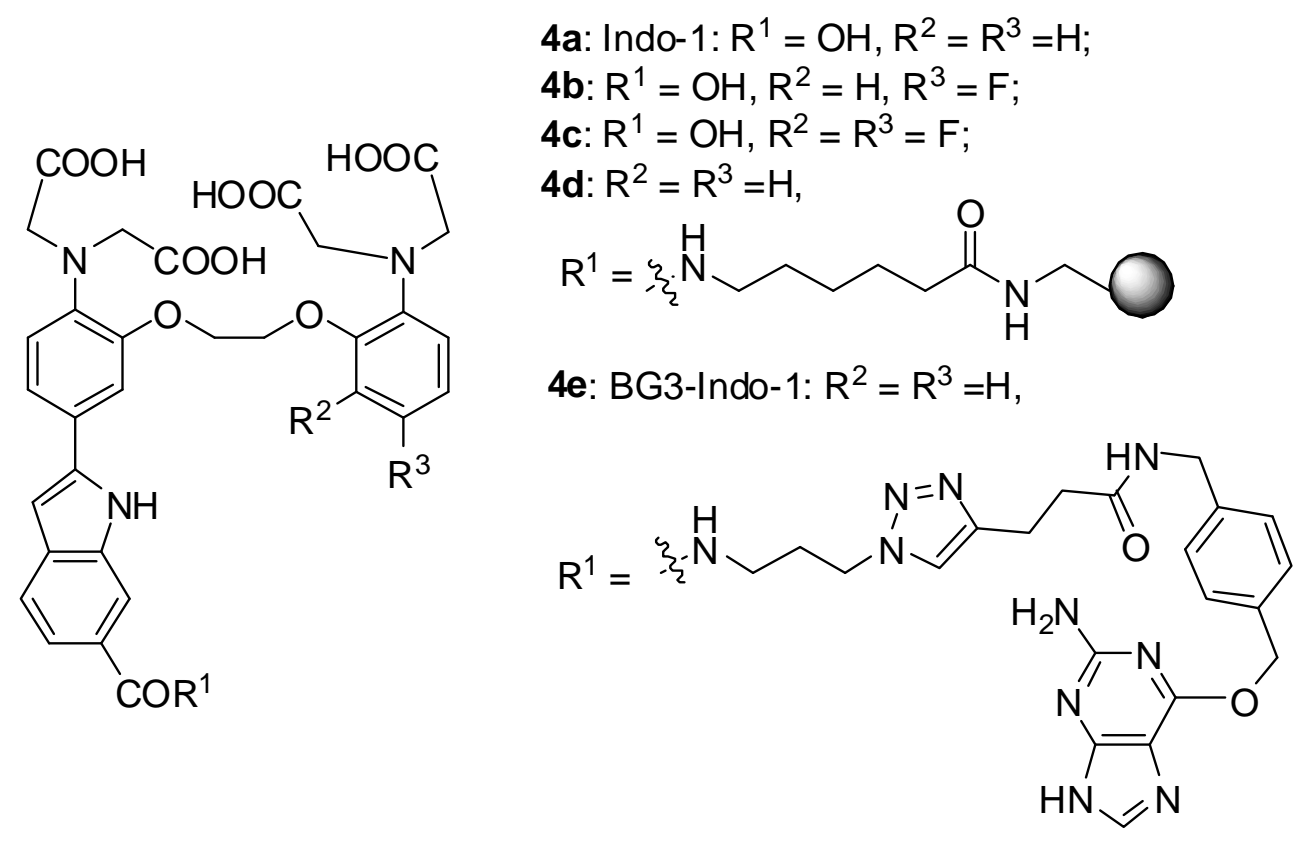

Figure 12. Indo-1 and various analogues. ${ }^{[35,39 b, 40-42]}$

Table 1. Fura-2 and its various analogues. ${ }^{[\mathrm{a}]}$

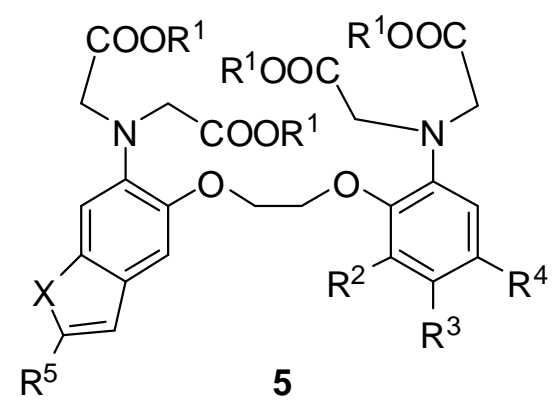

\begin{tabular}{|c|c|c|c|c|c|c|c|}
\hline No. & Nickname & $\mathrm{R}^{1}$ & $\mathrm{R}^{2}$ & $\mathrm{R}^{3}$ & $\mathrm{R}^{4}$ & $\mathrm{X}$ & $\mathrm{R}^{5}$ \\
\hline $5 a$ & Fura-2 & $\mathrm{H}$ & $\mathrm{H}$ & $\mathrm{Me}$ & $\mathrm{H}$ & $\mathrm{O}$ & \\
\hline $5 b$ & None & $\mathrm{H}$ & $\mathrm{H}$ & $\mathrm{Me}$ & $\mathrm{H}$ & $\mathrm{O}$ & \\
\hline $5 c$ & None & $\mathrm{H}$ & $\mathrm{H}$ & $\mathrm{Me}$ & $\mathrm{H}$ & $\mathrm{S}$ & \\
\hline
\end{tabular}




$$
\text { 5d None }
$$




5n Fura-5F $\quad \mathrm{K} \quad \mathrm{H} \quad \mathrm{F} \quad \mathrm{Cl}$

[a] Absorption and emission maxima for the free indicators and their complexes (No.: $\lambda_{a b s}^{\max } / \lambda_{f l}^{\max }-\lambda_{a b s}^{\max }($ complex $) / \lambda_{f l}^{\max }($ complex $)$, all values of wavelengths in $\left.\mathrm{nm}\right) ; \mathbf{5 a}: 362 / 512-$ $335 / 505 ;^{[35]}$ 5h: $363 /-340 / ;^{[38 \mathrm{a}]} \mathbf{5 i}: 364 / 508-335 / 495 ;^{[38 b]} \mathbf{5 j}$ : $364 / 502-335 / 490 ;^{[38 b]}$ 5k: $338 /-367 / ;^{[39 a, b]}$ 5l: $371 / 510-339 / 510 ;{ }^{[40]}$ 5m: $367 / 511-339 / 511 ;{ }^{[40]}$ 5n: $366 /$ $511-337 / 511 ;^{[40]}$ 5o: $367 / 510-337 / 510 .^{[40]}$

The drawback of the initial BAPTA derivatives was the necessity to work at $339 \mathrm{~nm}$ as an excitation wavelength. But below $\approx 360 \mathrm{~nm}$, the glass optics is not transparent enough, because it strongly absorbs UV light. Moreover, the autofluorescence of cells produces a high background signal, which leads to large errors in the determinations of $\mathrm{Ca}^{2+}$ concentrations. If the sensor brightness is high enough (which is not the case for Quin-2 with $\varepsilon \approx 5000$ and $\Phi_{f l} \approx 3-14 \%$ ), this problem may be circumvented. The new indicators $4 \mathbf{a}$ and $5 \mathbf{a}$ mentioned above have $\lambda_{a b s}^{\max }=349$ or $362 \mathrm{~nm}$, so that a red shift of 10-20 nm could be achieved, and the substances became more suitable for the available excitation sources in optical microscopy. Along this, the emission maxima were blue-shifted to 485 and $512 \mathrm{~nm}$ in Indo-1 and Fura-2, respectively, and the fluorescence quantum yields were found to be 0.38 and 0.23 , respectively. However, the calcium complexation provided smaller changes for the latter two sensors than for Quin-2 (the lower the "fluorescence jump", the lower the sensitivity of a sensor). This obstacle could be overcome by introducing the double ratiometric method of calcium determination. ${ }^{[35]}$ Another feature of compounds $\mathbf{4 a}$ and $\mathbf{5 a}$ is their higher $\mathrm{Ca}^{2+}$ affinity $\left(K_{\mathrm{D}}=250\right.$ and $224 \mathrm{nM}$, respectively), than for Quin-2, that allows to determine the $\mathrm{Ca}^{2+}$ concentration at lower levels in the medium. 
The high demand for compounds with properties similar to those of the $\mathrm{Ca}^{2+}$ sensors $4 \mathbf{a}$ and 5a stimulated further research on novel calcium indicators for elaborate applications. Fura-2 was one of the most popular lead structures. For instance, the change of the oxazol-5carboxylic acid part (compound 5a) to imidazolidine derivatives (Table 1, compounds 5b-d) or thiazolidines (Table 1, compounds 5e-g), and the variation of the heteroatom in the benzofuran ring enabled one to create indicators with red-shifted absorption bands ${ }^{[36]}$ (and thus to improve the set-up for the flow cytometry). As a result of these improvements, highpower UV lasers could be removed from optical set-ups. Cloned cells could now be analyzed, due to the absence of emitted light originating from the cloning reagent upon excitation with UV light.

One particular study strongly suggested, that the parameters of $\left[\mathrm{Ca}^{2+}\right]$ transients actually reached with Fura-2 turned out to be lower than the predicted ones. ${ }^{[37]}$ Eventually, a new derivative of Fura-2 (known also as $\mathbf{C}_{\mathbf{1 8}}$-Fura-2, $\mathbf{5 h}$ ) was synthesized. ${ }^{[38 \mathrm{a}]}$ The presence of a long alkyl chain resulted in the localization of this substance in the lipid bilayer of a cell membrane near the sites of calcium influx. However, compound $\mathbf{5 h}$ had some drawbacks. Its brightness was only a quarter of that of Fura-2, and the fluorescent signal was hard to distinguish from the background signal in the cell. Another drawback was the lower $K_{\mathrm{D}}$-value (ca. $150 \mathrm{nM}$ ) and, as a consequence, an earlier "saturation" of this sensor. Nevertheless, the initial rise in $\left[\mathrm{Ca}^{2+}\right]$ transients displayed by the membrane-associated $\mathbf{5 h}$ was found to be four to six times faster than that obtained with the bulk cytosolic fluorescent calcium indicators like Fura-2. One of the drawbacks of $\mathbf{5 h}$ was partially alleviated by creating the new sensor FFP18. ${ }^{[38 b]}$ Instead of a thiocarbonyl amino group, this compound had a propionic acid residue and a piperazine ring which serve as a bridge between the long alkyl chain and the aminophenyl group. Compound FFP18 displayed a $K_{\mathrm{D}}$ value that is twice as high as that of 5h (330 nM vs. $150 \mathrm{nM})$. This feature, as well as the long dodecyl residue, which can anchor the dye in the cell membrane, may provide more reasonable data on the parameters of calcium transients.

Another drawback of Fura-2 was found by molecular biologists. This substance rapidly leaked out of the cell or was transported to the vacuoles due to a specific mechanism of active transport of its anionic form. However, in the presence of positively charged groups, the behavior of this compound is different. Therefore, a new zwitterionic fragment based on a piperazine residue was incorporated into the structure of Fura-2 (see Table 1). ${ }^{[38 b]}$ To avoid changes in $\mathrm{pH}$ sensitivity, a propionic acid fragment was incorporated between the piperazine moiety and the aromatic rings in compounds $\mathbf{5 i}, \mathbf{5 j}$. The $K_{\mathrm{D}}$ value of the sensor $\mathbf{5 i}$ was $50 \%$ 
larger than that of Fura-2, and this indicator was hold back in cells quite well. The latter fact was confirmed by the low drift of the apparent baseline of internal $\left[\mathrm{Ca}^{2+}\right]$ : in 1 hour this line drifted 7 times more slowly than in the case of Fura-2.

With their new $\mathrm{Ca}^{2+}$ sensor TF-BAPTA (see Figure 10), London et al. ${ }^{[39 \mathrm{a}, \mathrm{b}]}$ introduced compounds with a new range of available $K_{\mathrm{D}}$ values. Compound TF-BAPTA showed a $K_{\mathrm{D}}$ value of about $65 \mu \mathrm{M}$, and allowed dual measurements of metal ion concentrations. This progress was achieved by the introduction of two fluorine atoms into each of the BAPTA rings. First of all, optical mode of measurements is the same as in the case of the common BAPTA sensor, when the concentration of calcium is determined by measuring the optical density. The second mode is based on the dependence of the ${ }^{19} \mathrm{~F}$ chemical shift of $\mathrm{R}^{2}=\mathrm{F}$ (compound $\mathbf{3 b}$, Figure 10) on the $\mathrm{Ca}^{2+}$ concentration. The variation of this chemical shift may be as large as $9.7 \mathrm{ppm}$. Unfortunately, as in the case of BAPTA, the sensor $\mathbf{3 b}$ cannot be used for the determination of calcium concentration by measuring the fluorescence signal. There are cases, for which indicators with low calcium affinities are required in biological studies. For instance, for studying cells or organelle systems with micromolar $\mathrm{Ca}^{2+}$ contents, or for the evaluation of very high variations in calcium concentration (e. g. by ischemia), a low affinity is required. For these applications the Fura-F sensor was designed and synthesized. It displays a $K_{\mathrm{D}}$ value of $20-30 \mu \mathrm{M}$ with lower sensitivity to $\mathrm{pH}$ (at physiological values of 5-8) than Fura-2, and low sensitivity to $\mathrm{Mg}^{2+}$ (even when $\left[\mathrm{Mg}^{2+}\right]=75 \mathrm{mM}$ ). The presence of two fluorine atoms in the "right-hand" phenyl ring $\left(\mathrm{R}^{4}\right.$ in compound $\mathbf{5 k}$, Table 1) makes it possible to use the chemical shift of one of them for an additional evaluation of the $K_{\mathrm{D}}$ value. The maximal span of the ${ }^{19} \mathrm{~F}$ chemical shift values was found to be $9.2 \mathrm{ppm}$.

The monohalogenated Fura-2 derivatives 5k-o were prepared in the search for indicators which possess $K_{\mathrm{D}}$ values intermediate between those of Fura-2 and Fura F. ${ }^{[40]}$ This indicator series shows no substantial deviation in the excitation and emission spectra compared to Fura-2. However, an influence of the position and nature of the halogen atom in the "righthand" phenyl ring (Table 1) on the $K_{\mathrm{D}}$ value was detected. The chlorine atom in compound $\mathbf{5 n}$ and the fluorine atom in compound $\mathbf{5 m}$ in the para-position to the amino group have the same influence on the $K_{\mathrm{D}}$ value (400 $\mathrm{nM}$ for both compounds $\mathbf{5 n}$ and $\mathbf{5 m}$ ). This value increases, when the fluorine atom is in the ortho-position to the ether oxygen as in compound $\mathbf{5} \mathbf{l}$, and drastically increases, when the fluorine atom occupies the para-position to the ether oxygen as in compound $\mathbf{5 0 .}$

Another calcium sensor (Indo-1) reported by Tsien et al. in 1985 was not widely used for elaborate optimization. However, its analogues with low calcium affinity were developed (e. 
g. the monofluorinated derivative $4 \mathbf{b}^{[40]}$ and the difluoroanalogue $4 \mathbf{c}^{[39 b]}$ in Figure 12). Compound $\mathbf{4 b}$ possesses a $K_{\mathrm{D}}$ value which is twice as high as that of Indo-1. A new modification of Indo-1 was introduced by Sanchez-Martin et al. ${ }^{[41]}$ It offered two advantages simultaneously: the delivery into and the preservation of the indicator in the cell. These advantages were achieved by the synthesis of the adduct of Indo-1 on aminomethylated polystyrene microspheres. The final product $4 \mathbf{d}$ (Figure 12) featured a $10 \mathrm{~nm}$ blue shift of the emission maximum upon calcium binding, and its $K_{\mathrm{D}}$ value is similar to that of Indo-1 (226 nM). "Leakage" of the sensor could not be detected, and no cell degradation was observed. The sensor 4d was not toxic to the cells and located in the cytosol without compartmentalization.

The other authors prepared an adduct of Indo-1 with $O$-(4aminomethylbenzyl)guanine, ${ }^{[42]}$ in order to use it as a specific marker for SNAP-tagged proteins, ${ }^{[43]}$ which can react with small cell-permeable molecules containing $O$-alkylguanine residues. On this basis, a new method for the selective detection and the study of calcium signals was elaborated. The SNAP-tagged protein was localized in the cell nucleus. Initially, compound 4e had an absorption maximum with a red shift of $10 \mathrm{~nm}$ compared to Indo-1. After binding compound $4 \mathrm{e}$ to the SNAP tag, the red shift decreased to $5 \mathrm{~nm}$. Although the fluorescence maximum of the free form of compound $4 \mathbf{e}$ was red-shifted (about $39 \mathrm{~nm}$ ) in comparison with that of the free form of compound $\mathbf{4 a}$, the fluorescence maximum of Cacomplexed 4e was the same as for the Ca-complexed 4a. The fluorescence maximum of the conjugate 4e with the SNAP tag did not change substantially both in the Ca-free form and the Ca-bound form in comparison with the corresponding values for compound 4a. The dissociation constant $K_{\mathrm{D}}$ of the calcium complex with $4 \mathbf{e}$ diminished by $30 \%$ in comparison with that of the calcium complex 4a. Fluorescence quantum yields $\Phi_{\mathrm{fl}}$ were two times lower for both forms of $\mathbf{4 e}$ (with and without calcium) compared to the corresponding values for compound 4a. Upon binding compound 4e with the SNAP tag, $K_{\mathrm{D}}$ and $\Phi_{\mathrm{fl}}$ values were recovered (completely or partially) up to those of compound 4a. The low fluorescence quantum yield of the free dye $\mathbf{4 e}$, together with the considerable increase in the fluorescence intensity occurring upon binding to the SNAP tag, increases the contrast of measurements. Besides that, an irreversible coupling of Indo-1 with the SNAP tag prevented the leakage and translocation of the indicator.

One of the main problems in the application of Fura-2 and Indo-1 in biological studies is the necessity to excite their fluorescence with UV light. In order to shift the main absorption band to the red, conventional dyes such as fluoresceins and rhodamines were combined with 
the BAPTA chelator. ${ }^{[4]}$ The later was attached to the phenyl substituent of these xanthene dyes.

Table 2. Xanthene-based calcium indicators. ${ }^{[\mathrm{a}]}$

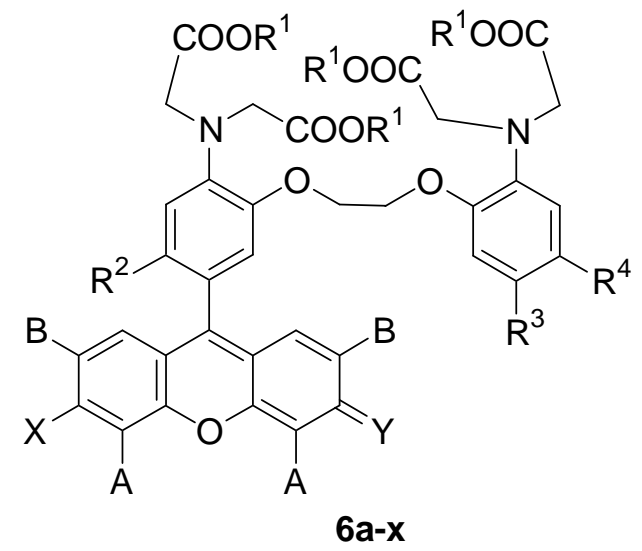

\begin{tabular}{|c|c|c|c|c|c|c|c|c|c|}
\hline No. & Nickname & $\mathrm{R}^{1}$ & $\mathrm{R}^{2}$ & $\mathrm{R}^{3}$ & $\mathrm{R}^{4}$ & A & B & $\mathrm{X}$ & Y \\
\hline $6 \mathbf{a}$ & Rhod-1 & $\mathrm{K}$ & $\mathrm{OH}$ & $\mathrm{Me}$ & $\mathrm{H}$ & $\mathrm{H}$ & $\mathrm{H}$ & $\mathrm{NMe}_{2}$ & ${ }^{+} \mathrm{NMe}_{2}$ \\
\hline $6 b$ & Rhod-2 & $\mathrm{H}$ & $\mathrm{H}$ & $\mathrm{Me}$ & $\mathrm{H}$ & $\mathrm{H}$ & $\mathrm{H}$ & $\mathrm{NMe}_{2}$ & ${ }^{+} \mathrm{NMe}_{2}$ \\
\hline $6 c$ & Fluo-1 & $\mathrm{H}$ & $\mathrm{OH}$ & $\mathrm{Me}$ & $\mathrm{H}$ & $\mathrm{H}$ & $\mathrm{H}$ & $\mathrm{OH}$ & $\mathrm{O}$ \\
\hline $6 d$ & Fluo-2 & $\mathrm{H}$ & $\mathrm{H}$ & $\mathrm{Me}$ & $\mathrm{H}$ & $\mathrm{H}$ & $\mathrm{H}$ & $\mathrm{OH}$ & $\mathrm{O}$ \\
\hline $6 e$ & Fluo-3 & $\mathrm{H}$ & $\mathrm{H}$ & $\mathrm{Me}$ & $\mathrm{H}$ & $\mathrm{H}$ & $\mathrm{Cl}$ & $\mathrm{OH}$ & $\mathrm{O}$ \\
\hline $6 f$ & Fluo-4 & $\mathrm{H}$ & $\mathrm{H}$ & $\mathrm{Me}$ & $\mathrm{H}$ & $\mathrm{H}$ & $\mathrm{F}$ & $\mathrm{OH}$ & $\mathrm{O}$ \\
\hline $6 \mathrm{~g}$ & Fluo-5F & $\mathrm{H}$ & $\mathrm{H}$ & $\mathrm{F}$ & $\mathrm{H}$ & $\mathrm{H}$ & $\mathrm{F}$ & $\mathrm{OH}$ & $\mathrm{O}$ \\
\hline $6 h$ & Fluo-5Cl & $\mathrm{H}$ & $\mathrm{H}$ & $\mathrm{Cl}$ & $\mathrm{H}$ & $\mathrm{H}$ & $\mathrm{F}$ & $\mathrm{OH}$ & $\mathrm{O}$ \\
\hline $6 \mathbf{i}$ & Fluo-5N & $\mathrm{H}$ & $\mathrm{H}$ & $\mathrm{NO}_{2}$ & $\mathrm{H}$ & $\mathrm{H}$ & $\mathrm{F}$ & $\mathrm{OH}$ & $\mathrm{O}$ \\
\hline $6 \mathbf{j}$ & None & $\mathrm{H}$ & $\mathrm{H}$ & $\mathrm{H}$ & xtran & $\mathrm{H}$ & $\mathrm{F}$ & $\mathrm{OH}$ & $\mathrm{O}$ \\
\hline $6 \mathbf{k}$ & None & $\mathrm{H}$ & $\mathrm{H}$ & $\mathrm{H}$ & $\mathrm{CH}_{2} \mathrm{COOH}$ & $\mathrm{H}$ & $\mathrm{F}$ & $\mathrm{OH}$ & $\mathrm{O}$ \\
\hline 61 & $\begin{array}{l}\text { Fluo-4- } \\
\text { dextran }\end{array}$ & K & $\mathrm{H}$ & $\mathrm{H}$ & & $\mathrm{H}$ & $\mathrm{F}$ & $\mathrm{OH}$ & $\mathrm{O}$ \\
\hline $6 m$ & $\begin{array}{l}\text { Fluo-4- } \\
\text { cadaverine }\end{array}$ & $\mathrm{K}$ & $\mathrm{H}$ & $\mathrm{H}$ & ${ }^{5}{ }^{\mathrm{CONH}}+\mathrm{Y}_{5} \mathrm{NH}_{2}$ & $\mathrm{H}$ & $\mathrm{F}$ & $\mathrm{OH}$ & $\mathrm{O}$ \\
\hline
\end{tabular}




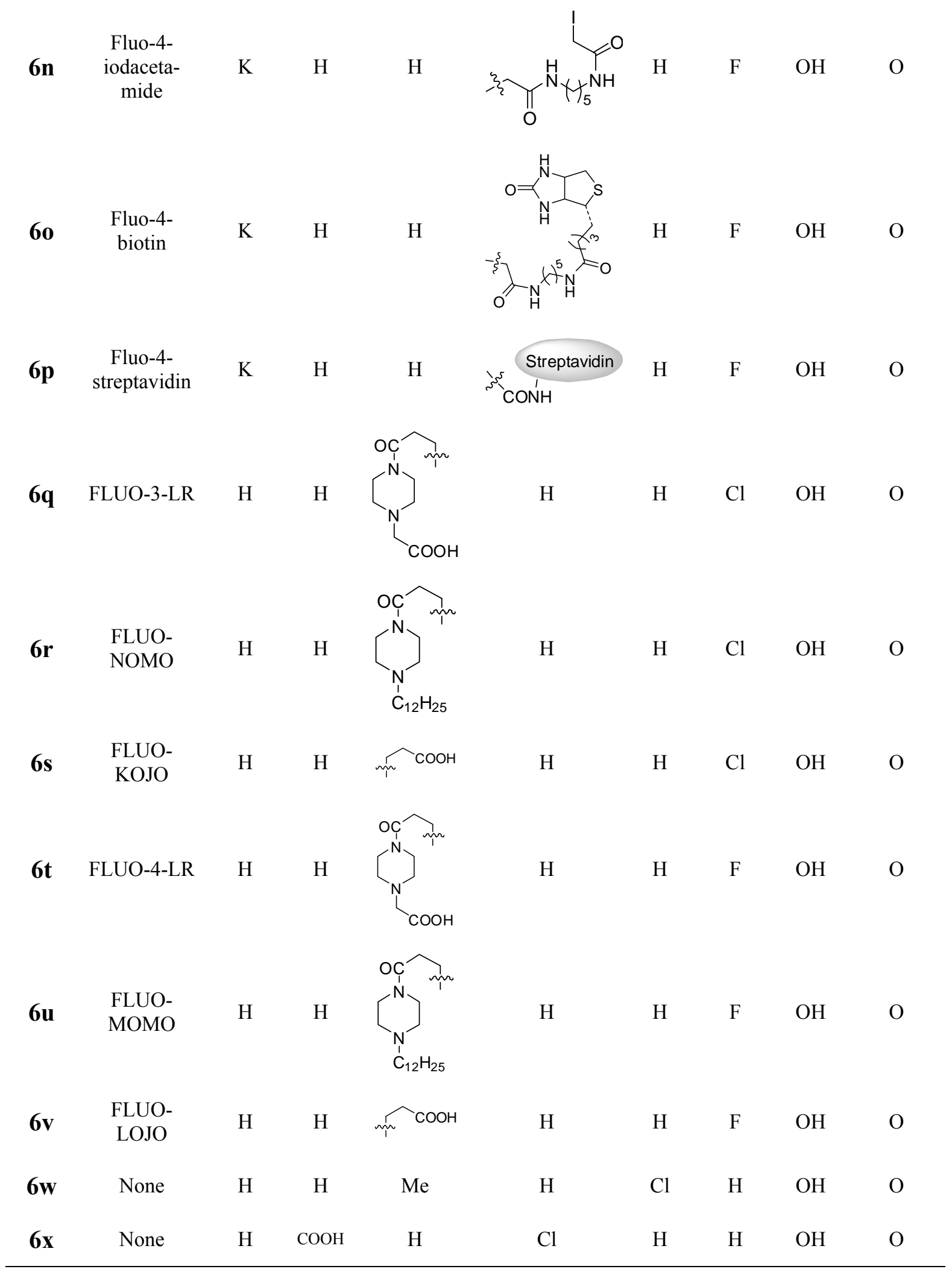




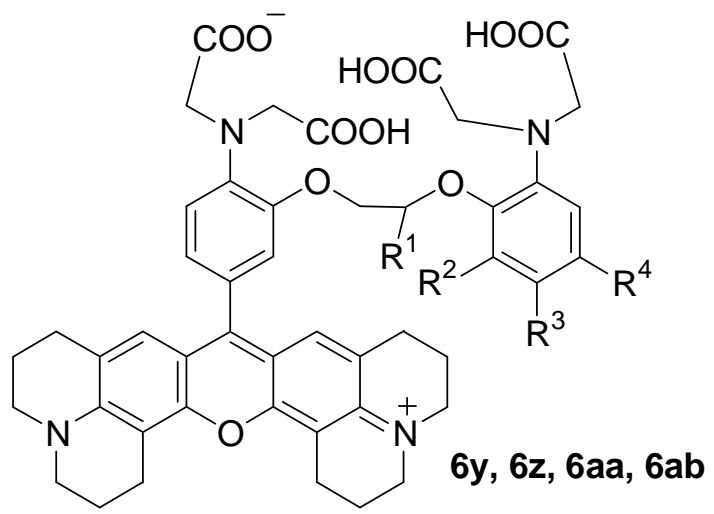

\begin{tabular}{cccccc}
\hline No. & Nickname & $\mathrm{R}^{1}$ & $\mathrm{R}^{2}$ & $\mathrm{R}^{3}$ & $\mathrm{R}^{4}$ \\
\hline $\mathbf{6 y}$ & X-Rhod-1 & $\mathrm{H}$ & $\mathrm{H}$ & $\mathrm{Me}$ & $\mathrm{H}$ \\
$\mathbf{6 z}$ & X-Rhod-5F & $\mathrm{H}$ & $\mathrm{H}$ & $\mathrm{F}$ & $\mathrm{H}$ \\
$\mathbf{6 a a}$ & X-Rhod-FF & $\mathrm{H}$ & $\mathrm{F}$ & $\mathrm{F}$ & $\mathrm{H}$ \\
$\mathbf{6 a b}$ & $\begin{array}{c}\text { Calcium } \\
\text { Ruby-Cl }\end{array}$ & $\mathrm{N}_{3}$ & $\mathrm{H}$ & $\mathrm{H}$ & $\mathrm{Cl}$ \\
\hline
\end{tabular}

[a] The values of absorption and emission maxima for free indicators and their complexes (Nr.: $\lambda_{a b s}^{\max } / \lambda_{f l}^{\max }-\lambda_{a b s}^{\max }($ complex $) / \lambda_{f l}^{\max }($ complex $)$, all values of wavelengths in $\left.\mathrm{nm}\right) ; \mathbf{6 a}: 556 /$ 578 - $556 / 578 ; ;^{[44]}$ 6b: $553 / 576$ - $553 / 576 ;{ }^{[44]}$ 6c: 499 / $521-499 / 521 ; ;^{[44]}$ 6d: 493 / 518 493 / 518; ${ }^{[44]}$ 6e: 506 / 526 - $506 / 526 ;{ }^{[44]}$ 6f: 494 / 516 - 494 / 516; ${ }^{[47]}$ 6g: $491 / 516$ - 494 / 516; ${ }^{[47]}$ 6h: $491 / 516$ - $494 / 516 ;^{[47]} \mathbf{6 i}: 491 / 516-494 / 516 ;{ }^{[47]} \mathbf{6 j}: 495 / 517-495 / 517 ;^{[48]}$ 61: 496 / $520-496 / 520 ;{ }^{[48]} \mathbf{6 m}: 495 / 518-495 / 518 ;{ }^{[48]}$ 6n: 494 / $517-494 / 517 ;{ }^{[48]}$ 6o: 495 / 518 - 495 / 518; ${ }^{[48]}$ 6p: 495 / $518-495 / 518 ;{ }^{[48]}$ 6s: / $526-/ 526 ;^{[49]}$ 6v: / $515-/$ 515; ${ }^{[49]}$ 6w: / 540 - / 540; ${ }^{[50]}$ 6x: $364 / 517$ - $335 / 517,{ }^{[51]}$ 6y: $580 / 602-580 / 602 ;{ }^{[8]}$ 6ab: $579 / 598-579 / 598 ;^{[52]}$

As a result, the new series of calcium sensors 6a-e emerged, with absorption maxima in the region of $500 \mathrm{~nm}$ (Fluo-1, Fluo-2, Fluo-3) to $550 \mathrm{~nm}$ (Rhod-1, Rhod-2), with Stokes shifts of about 20-25 $\mathrm{nm}$. These spectroscopic properties of the xanthene-based indicators could not interfere with natural fluorescence of cell components (autofluorescence) and allowed to work simultaneously with UV-photolyzable calcium chelators (see Figure 13) like nitr-5, nitr-7, ${ }^{[45]}$ and DM-nitrophen. ${ }^{[46]}$ 

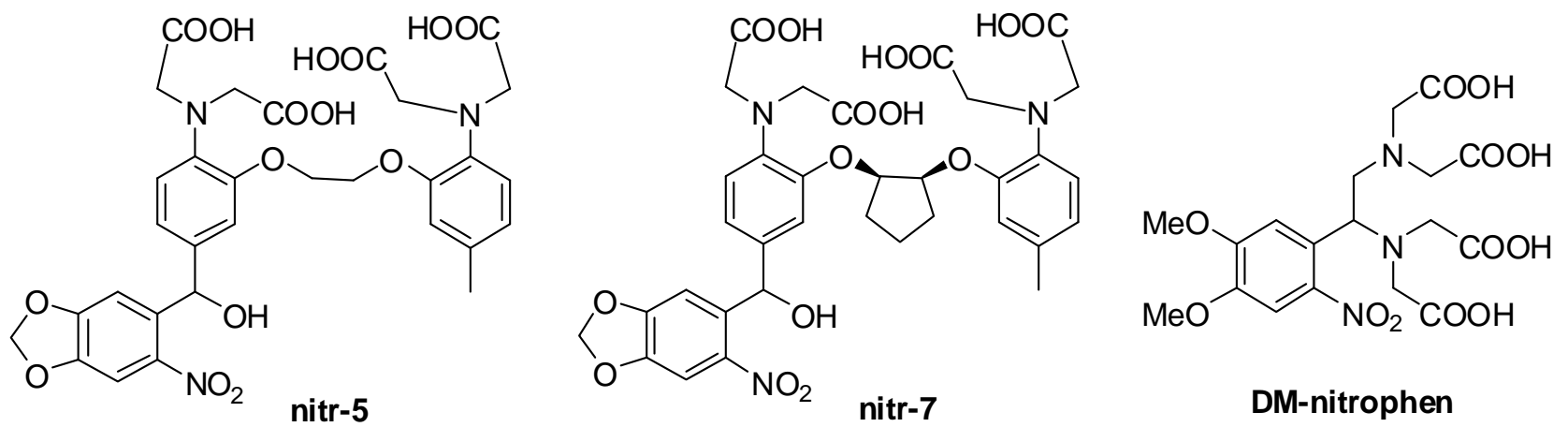

Figure 13. Photolyzable calcium chelators for a controlled release of calcium cations upon illumination with UV light. The irradiation destroyes the polydentate ligand by cleaving the nitropiperonyl or the nitroveratryl groups.

The higher dissociation constants of the Fluo- and the Rhod-sensors (compared to Fura-2) have improved the resolution of different concentrations in the micromolar range of intracellular calcium ( $K_{\mathrm{D}}$ for Fluo-1, Fluo-2, and Fluo-3 - 0.7, 0.37, 0.40 and for Rhod-1, Rhod-2 - 2.3, $1.0 \mu \mathrm{M}$, respectively). The high increase in fluorescence intensity upon cation coordination (40-fold for Fluo-3) enhanced the sensitivity to calcium in the whole interval of concentrations $\left(0.1 K_{\mathrm{D}}\right.$ to $\left.10 K_{\mathrm{D}}\right)$. In the cases of high and low calcium concentrations, sensitivity is not lost. However, this series of indicators had the same drawback as Quin-2, it provided only low brightness. Apart from that, the shift of the fluorescence maxima of xanthene sensors upon calcium complexation was too small, and they cannot be used for ratiometric measurements. In this case, in order to evaluate the absolute value of a local calcium concentration, some information on local dye concentration is required, as well as optical path length and instrument sensitivity. However, two promising xanthene indicators emerged. The first one - Rhod-2 - possessed a mitochondrial localization as a valuable feature. The second one - Fluo-3 - had a lower pH sensitivity than related compounds (e. g. Fluo-1 and Fluo-2).

Fluorinated analogues of Fluo-3 were developed by K. Gee and co-workers. ${ }^{[4]}$ The new indicator Fluo-4 (compound 6f, see Table 2) is a brighter fluorescent dye in vitro and in vivo compared to compound Fluo-3 (upon excitation with an argon laser at $488 \mathrm{~nm}$ ). As has already been mentioned, the brightness of a fluorescent dye is the product of the extinction coefficient $(\varepsilon)$ and the fluorescence quantum yield $\left(\Phi_{\mathrm{fl}}\right)$. Since the $\Phi_{\mathrm{fl}}$ values and the position of the emission maxima of compounds $6 \mathbf{e}$ and $\mathbf{6 f}$ are similar, and the $\varepsilon$ value at the "working" wavelength of $488 \mathrm{~nm}$ is 1.8 times higher for the indicator $\mathbf{6 f}$, compound $\mathbf{6 f}$ gives brighter pictures than 6e. It is possible to use a lower concentration of a "brighter" dye to gain the same signal intensity. Low dye concentrations reduce buffering effects and the amount of 
toxic photolysis products. Brighter dyes provide the possibility to use lower illumination intensities with the same dye concentration. Lower illumination intensities are advantageous as they cause lower photobleaching of the indicator. In addition, by replacing the methyl group at the positions of $\mathrm{R}^{3}$ with acceptor groups, the series of Fluo-4 analogues (6g-i) was prepared. They possess similar spectral properties, but their calcium affinities are lower than that of Fluo-4 $\left(6 \mathbf{g}-2.3 \mu \mathrm{M}, 6 \mathbf{h}-6.2 \mu \mathrm{M}, 6 \mathbf{i}-90 \mu \mathrm{M}\right.$; for comparison, the $K_{\mathrm{D}}$ of Fluo-4 is $0.35 \mu \mathrm{M}) . K_{\mathrm{D}}$ values of compounds $\mathbf{6 g}-\mathbf{i}$ correspond to physiological $\mathrm{Ca}^{2+}$ levels.

Perfect photophysical properties made the indicator Fluo-4 to be one of the main candidates for further improvements. The first attempt - compound $\mathbf{6 j}$ - was not a success. ${ }^{[48]}$ A carboxy group was introduced into the para-position relative to the ether oxygen atom in the structure of compound $\mathbf{6 e}$, which caused a significant decrease in calcium affinity of the resulting derivative $\mathbf{6 j}$. The second derivate of Fluo-4 - compound $\mathbf{6} \mathbf{k}^{[48]}$ - displayed higher calcium affinity (probably due to the presence of the methylene bridge between the aromatic ring and the carboxy group). Eventually, the adduct of compound $\mathbf{6 k}$ with dextran compound $6 \mathbf{l}$ - was prepared. Dextran, as a voluminous fragment, increases the retention time of $\mathbf{6} \mathbf{l}$ in living cells and improves the intracellular protein binding. However, compound $\mathbf{6} \mathbf{l}$ has a serious disadvantage: the low $\Phi_{\mathrm{fl}}$ value (ca. $3 \%$ at saturation with calcium and ca. $0.2 \%$ in its absence); these values were even lower than for Quin-2 in both forms. A series of further conjugates were synthesized from compound $\mathbf{6 k}$, which could label other cell compartments: a) fluo-4-cadaverine $\mathbf{6 m}$ for labeling of proteins through the activated carboxylate groups; $b$ ) compound $\mathbf{6 m}$ for the labeling of ribonucleotide after periodate cleavage; c) a thiol-reactive compound called fluo-4-iodoacetamide $\mathbf{6 n}$ for cysteine residues; d) biotinylated indicator fluo-4-biotin 60 for obtaining a very stable biotin-(strept)avidin complex. In order to confirm the utility of $\mathbf{6 o}$, the streptavidin complex $\mathbf{6 p}$ of this compound was prepared. The whole series of Fluo-4 derivatives had very similar fluorescence and absorption spectra, but their calcium affinities and fluorescence enhancements upon calcium coordination are lower than those of Fluo-4.

Similar problems - leakage and compartmentalization ${ }^{[38 b]}$ - were again encountered by the same authors nine years later, and were solved by the same methods. ${ }^{[49]}$ Sensors FLUO-3LR and FLUO-4-LR (see Table 2), elaborated analogues of Fluo-3 and Fluo-4, respectively, were prepared. Comparing the sensor structures in Tables 1 and 2, one can notice that the modification of the sensors was carried out in exactly the same manner as in the previously described compound Fura-2, which was replaced by compound PE3 (see Table 1). Derivatives with a long hydrocarbon chain (compounds $\mathbf{6 r}$ and $\mathbf{6 u}$, see Table 2) were also 
synthesized to monitor the near membrane calcium transients. Two interesting Fluo analogues were disclosed: FLUO-KOJO and FLUO-LOJO (see Table 2). ${ }^{[49]}$ Both compounds are the precursors to other optimized indicators $(\mathbf{6 q - r}, \mathbf{6 t - u})$, and both contain only a propionic acid residue, but do not contain a piperazine fragment as do compounds $\mathbf{6 q - r}, \mathbf{6 t - u}$. Nevertheless, according to the report, ${ }^{[49]}$ they show a "general leakage resistance" (50\% less leakage of FLUO-LOJO in one hour compared to Fluo-4).

J. Han et al. prepared compound 6w, the analogue of Fluo-3 with different positions of chlorine atoms (Table 2). ${ }^{[50]}$ This new indicator $\mathbf{6 w}$ had a slightly more red-shifted excitation maximum (540 nm vs. $526 \mathrm{~nm}$ in Fluo-3), and displayed a 9-fold increase in fluorescence signal upon changing the calcium concentration from 0 to $50 \mu \mathrm{M}$.

An enormous number of potentially useful calcium sensors have been described by Diwu and co-workers. ${ }^{[51]}$ They were built on the basis of BAPTA and various fluorophores (xanthenes, acridines, carbopyranines). Unfortunately, the functional properties of these indicators were demonstrated only in one plot with the fluorescence spectrum of one compound (6x), a derivative of Fluo-2 with a carboxy group as $\mathrm{R}^{2}$ (see Table 2). The structural similarity with Fluo-2 explains the presence of the highly fluorescent form of $\mathbf{6 x}$ upon "calcium saturation" (at $0.5 \mathrm{mM}$ ) with a fluorescence maximum at $517 \mathrm{~nm}$ and the absence of a fluorescence signal without calcium cations. The same authors also observed a more than 100-fold increase in the fluorescence signal upon calcium binding for the whole series of Fluo-2, Fluo-3 and Fluo-4 derivatives (partially with various substituents $\mathrm{R}^{2}$, or acetoxymethylated derivatives of the parent indicators).

Indicators which absorb green or red light, allow one to neglect autofluorescence, and offer the possibility to simultaneously use calcium indicators and photoactivable calcium chelators. For example, X-Rhod-1 (6y) was created by Molecular Probes Inc. ${ }^{[9]}$ In comparison with the nearest analogue Rhod-2, the excitation/emission maxima of compound 6y were red-shifted by ca. $26 \mathrm{~nm}$ to $580 / 602 \mathrm{~nm}$, and the calcium affinity was increased by $30 \%$ (to $700 \mathrm{nM}$ ). This indicator (X-Rhod-1) was applicable for the simultaneous detection of $\mathrm{Ca}^{2+}$ transients and green-fluorescent protein (GFP) labeling with a minimal crosstalk. Fluorinated analogues of $\mathbf{6 y}$ - X-Rhod-5F (6z) and X-Rhod-FF (6aa) - have similar photophysical properties as compound X-Rhod-1, and can be used for measurements of various calcium levels, according to their increased dissociation constants $(1.6,17 \mu \mathrm{M}$, respectively). ${ }^{[9]}$

Another indicator, 6ab (see Table 2), with similar photophysical properties and structure as X-Rhod-1, was prepared by Gaillard and co-workers. ${ }^{[52]}$ However, compound 6ab had 
some important changes in structure which improved its performance (in comparison with $\mathbf{X}$ Rhod-1). An aliphatic chain with a terminal azido group was introduced. The azido alkyl residue was connected with the ethylene-1,2-dioxy bridge of the BAPTA fragment. A chlorine atom was also introduced in the para-position relative to the ether bridge. The position of the azidoalkyl moiety was chosen in such a way that it is "less interfering with the functional part of the indicator". ${ }^{[52]}$ Indeed, it resulted in a sharp increase of the dissociation constant from 0.7 (for $\mathbf{X}$-Rhod-1) to $30.3 \mu \mathrm{M}$. Undoubtedly, at least a part of this increase is due to the effect of the chlorine atom, but not the whole increase, as the authors considered. ${ }^{[52]}$ Besides that, the azido group provided additional possibilities to link this indicator to various objects (e. g. dextran, colloidal semiconductor nanocrystals), either by using "click chemistry" with acetylene-tagged molecules, or by Staudinger ligation, or by EDC-assisted coupling with any carboxy group (after reduction of an azido to an amino group). It should be noted, that the authors have not given a correct evaluation of the efficiency of the indicator Calcium Ruby-Cl (6ab). ${ }^{[52]}$ Usually, the efficiency of an indicator correlates with the value for the fluorescence ratio. The fluorescence ratio is the ratio of fluorescence quantum yields of the indicator in the free and the complexed form. However, for their indicator 6aa the authors $^{[51]}$ only estimate the quality on the basis of the ratio of the absolute intensity of the fluorescence emission maxima of the free and the complexed form of $\mathbf{6 a b}$. Thus, the authors claimed a higher performance of $\mathbf{6 a b}$, in comparison with Rhod-2, ${ }^{[4 \mathrm{bb}]}$ although they compared two "uncomparable" ratios $\left(I_{\mathrm{Ca}^{2+}-\text { free }}^{f l} / I_{\mathrm{Ca}^{2+} \text {-bound }}^{f l}\right.$ for $\mathbf{6 a a}$ and $\Phi_{\mathrm{Ca}}{ }^{2+}{ }_{\text {-free }} / \Phi_{\mathrm{Ca}}{ }^{2+}$-bound for Rhod-2), since fluorescence intensity is proportional not only to the fluorescence quantum yield, but also depends on the absorbance in the indicator solutions.

Unlike the Indo and Fura series, calcium indicators based on a xanthene fluorophore also enabled to shift the absorption bands to the red. However, xanthene-based sensors also have a drawback: there is no possibility to do ratiometric measurements with them. To circumvent this problem, another class of fluorophores - coumarines - was used. ${ }^{[53]}$

Table 3. Coumarine-based calcium indicators. ${ }^{[\mathrm{a}]}$ 


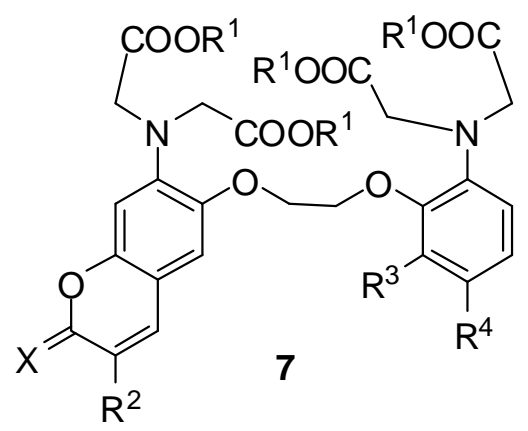

\begin{tabular}{|c|c|c|c|c|c|c|}
\hline No. & Nickname & $\mathrm{R}^{1}$ & $\mathrm{R}^{2}$ & $\mathrm{R}^{3}$ & $\mathrm{R}^{4}$ & $X$ \\
\hline $7 \mathbf{a}$ & $\mathrm{MCC}$ & $\mathrm{H}$ & $\mathrm{COMe}$ & $\mathrm{H}$ & $\mathrm{Me}$ & $\mathrm{O}$ \\
\hline $7 b$ & MTC & $\mathrm{H}$ & COOMe & $\mathrm{H}$ & $\mathrm{Me}$ & $\mathrm{O}$ \\
\hline $7 \mathrm{c}$ & $\mathrm{BXC}$ & $\mathrm{H}$ & & $\mathrm{H}$ & $\mathrm{Me}$ & $\mathrm{O}$ \\
\hline $7 d$ & BTC & $\mathrm{H}$ & & $\mathrm{H}$ & $\mathrm{Me}$ & $\mathrm{O}$ \\
\hline $7 e$ & No & $\mathrm{H}$ & & $\mathrm{F}$ & $\mathrm{F}$ & $\mathrm{O}$ \\
\hline $7 f$ & BTIC & $\mathrm{Li}$ & & $\mathrm{H}$ & $\mathrm{Me}$ & $\mathrm{NH}$ \\
\hline $7 \mathrm{~g}$ & BXIC & $\mathrm{Li}$ & & $\mathrm{H}$ & $\mathrm{Me}$ & $\mathrm{NH}$ \\
\hline $7 \mathrm{~h}$ & BIIC & $\mathrm{Li}$ & & $\mathrm{H}$ & $\mathrm{Me}$ & $\mathrm{NH}$ \\
\hline $7 \mathbf{i}$ & $\mathrm{C}_{12}$-BIIC & $\mathrm{Li}$ & & $\mathrm{H}$ & $\mathrm{Me}$ & $\mathrm{NH}\left(\mathrm{CH}_{2}\right)_{11} \mathrm{CH}_{3}$ \\
\hline $7 \mathbf{j}$ & $\mathrm{C}_{12}$-BTIC & $\mathrm{Li}$ & & $\mathrm{H}$ & $\mathrm{Me}$ & $\mathrm{NH}\left(\mathrm{CH}_{2}\right)_{11} \mathrm{CH}_{3}$ \\
\hline $7 \mathbf{k}$ & KCM-1 & $\mathrm{H}$ & $\mathrm{COOH}$ & $\mathrm{H}$ & $\mathrm{F}$ & $\mathrm{O}$ \\
\hline
\end{tabular}


[a] Absorption and emission maxima for free indicators and their complexes in the form: No.: $\lambda_{a b s}^{\max } / \lambda_{f l}^{\max }-\lambda_{a b s}^{\max }($ complex $) / \lambda_{f l}^{\max }($ complex $)$, all values of wavelengths in nm; 7a: $444 / 510-$ 390 / 510; $;^{[53]} \mathbf{7 b}: 405 / 480$ - 356 / 480; ${ }^{[53]} \mathbf{7 c}: 447$ / 510 - 415 / 510; ${ }^{[53]} \mathbf{7 d :} 462$ / 525 - $401 /$ $525 ;^{[53]} \mathbf{7 e}: 462 / 525$ - $401 / 525 ;^{[54]} \mathbf{7 f :} 471 / 535$ - $414 / 535 ;^{[55]} \mathbf{7 g}: 471 / 521$ - 409 / $521 ;^{[55]} \mathbf{7 h}: 469 / 519-401 / 499 ;^{[55]} \mathbf{7 j}: 470 / 530-421 / 530 ;^{[56]} \mathbf{7 k}: 403 / 480-358 /$ $475 ;^{[57]}$

By variation of the substituents $\mathrm{R}^{2}$ (Table 3, compounds 7a-d), H. Iatridou et al. managed to obtain the very interesting and promising compound $\mathbf{7 d}$. This new indicator has a fluorophore, which may be excited with visible light (462 nm). Moreover, it has a big "blue" shift of the absorption maximum $(61 \mathrm{~nm})$ upon calcium coordination (the non-shifted emission maximum is detected at $525 \mathrm{~nm}$ ). At the time of publication, indicator $7 \mathbf{d}$ possessed the highest dissociation constant $(7 \mu \mathrm{M})$. However, its fluorescence quantum yield was lower than that of Quin-2; the values for the "free" and "complexed" forms were 0.07 and 0.12 , respectively.

Compound 7e, a fluorinated analogue of BTC (7d), was invented by K. Takesako and coworkers. ${ }^{[54]}$ Indicator $7 \mathbf{e}$ has the same photophysical properties as its analogues, and an extraordinarily low affinity to calcium $(550 \mu \mathrm{M})$. This property facilitates the measurements of $\mathrm{Ca}$ levels in the endoplasmatic reticulum.

To increase the polarity of BTC 7d and provide the possibility for structural variations, a series of imino analogues of this compound were synthesized. ${ }^{[55]}$ Introduction of the imino group was accompanied by an improvement of calcium sensitivity (fluorescence increase for $7 \mathbf{f}$ was five times stronger than that of BTC, a decrease in the dissociation constants (e. g. $27.5 \mu \mathrm{M}$ for $\mathbf{7 h}$ ), moderate bathochromic shifts of the absorption and emission maxima (up to $10 \mathrm{~nm}$ ), shifts of the fluorescence maxima upon calcium coordination, e. g. for compound $\mathbf{7 h}$ by $20 \mathrm{~nm}$. The dodecyl analogues of BTIC and BIIC - $\mathrm{C}_{12}$-BTIC and $\mathrm{C}_{12}$-BIIC - were prepared later. ${ }^{[56]}$ The main advantage of these coumarine calcium indicators is their near membrane localization combined with a high dissociation constant $(5.50 \mu \mathrm{M}$ for $7 \mathbf{i}, 4.49 \mu \mathrm{M}$ for $7 \mathbf{j}$ ). Initially, the calcium sensors for measurements in inner-membrane vicinity possessed an affinity in the hundred nanomolar range. Sensitivity to calcium, in comparison with the non-dodecylated analogues (BTIC and BIIC), increased by a factor of two for 7i and decreased by more than a factor of five for $\mathbf{7 j}$.

A monofluorinated analogue of compound 7a was prepared by $\mathrm{H}$. Komatsu and coworkers. ${ }^{[57]}$ The new indicator $7 \mathbf{k}$ has a low affinity to cations $\left(K_{\mathrm{D}}\left(\mathrm{Ca}^{2+}\right)-14 \mu \mathrm{M}, K_{\mathrm{D}}\left(\mathrm{Mg}^{2+}\right)\right.$ 
- $26 \mathrm{mM}$ ), a small shift of the fluorescence maximum upon metal ion coordination (free ligand: $480 \mathrm{~nm}, \mathrm{Ca}^{2+} / \mathrm{Mg}^{2+}:-5 /+5 \mathrm{~nm}$ ) and a big shift of the absorption maximum (free ligand: $\left.403 \mathrm{~nm}, \mathrm{Ca}^{2+} / \mathrm{Mg}^{2+}:-45 /+21 \mathrm{~nm}\right)$. The calcium coordination causes a decrease in absorption and fluorescence intensities, the opposite effect was observed upon magnesium coordination - an increase in absorbtion and fluorescence intensities. The authors claimed, that the sensitivity of this compound to two analytes $\left(\mathrm{Mg}^{2+}\right.$ and $\left.\mathrm{Ca}^{2+}\right)$ is an advantage, and the different directions of response is a feature which helps to separate the magnesium and calcium signals. However, it should be noted, that in almost all of the above mentioned articles, the authors have presented an opinion exactly opposite to that. ${ }^{[32,35,44]}$ Usually the presence of magnesium ions influences the determination of the calcium concentration, because they also input into the background fluorescence signal. Any shift of the spectroscopic maxima (one from the other) of indicator solutions upon calcium and magnesium coordination may help to separate the magnesium signal away from the calcium signal, but only after complex calculations. Thus, the sensitivity of compound $\mathbf{7 k}$ to calcium and magnesium ions makes the precise measurements of the $\mathrm{Ca}$ content in living cells next to impossible. The main reason is the presence of both cations in the cells. However, for in vitro experiments, the indicator $7 \mathbf{k}$ can be suitable.

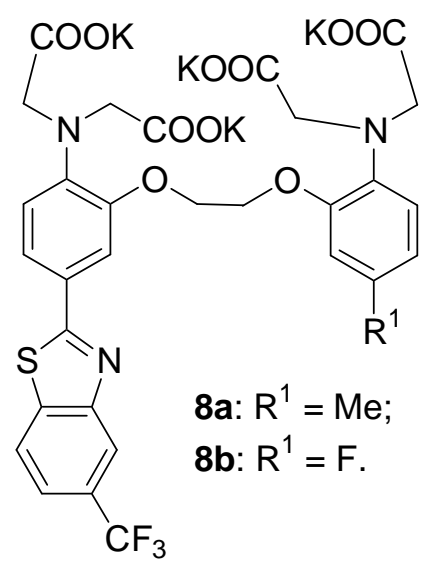

Figure 14. Indicators for calcium with a phenylbenzothiazole fluorophore. ${ }^{[40]}$

The benzothiazolo-BAPTA derivatives $\mathbf{8 a , b}$, with a shorter conjugated chain (in comparison with BTC) was prepared by K. R. Gee et al. ${ }^{[40]}$ Such a cut of the conjugated chain caused a $100 \mathrm{~nm}$ blue shift of the excitation maximum in comparison with BTC. Calcium complexation provides only a $36 \mathrm{~nm}$ blue shift of the absorption maximum (almost two times lower than the value of BTC). However, the new indicators were twice as bright as BTC. Moreover, the required binding affinity $(1.4 \mu \mathrm{M}$ for $\mathbf{8 b})$ was achieved. 
A completely different approach was pursued in the preparation of the so-called "Green" series of indicators by Molecular Probes Inc. ${ }^{[9]}$ Instead of including the fluorophore into the BAPTA chelator, these parts were separated by a phenylcarboxamide group. This change made the preparation easier, because all indicators have the same chelator. The separation of the BAPTA chelator from the fluorophore part led to a 5-fold increase in the fluorescence quantum yield (9a: 0.75, 9c: 0.7, in comparison with Fluo-3).

Table 4. "Green" series of calcium indicators. ${ }^{[a]}$

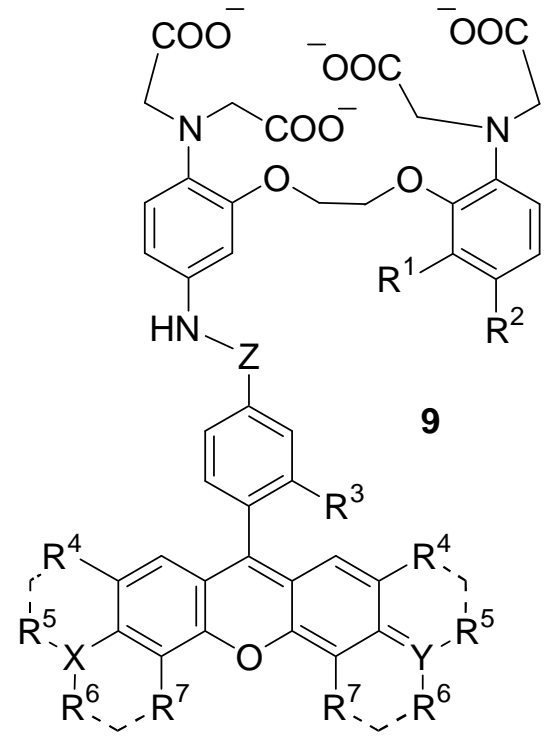

\begin{tabular}{|c|c|c|c|c|c|c|c|c|c|c|c|c|}
\hline No. & Nickname & $\begin{array}{c}K_{\mathrm{D}} \\
\left(\mathrm{Ca}^{2+}\right) \\
\mu \mathrm{M}\end{array}$ & $\mathrm{R}^{1}$ & $\mathrm{R}^{2}$ & $\mathrm{R}^{3}$ & $\mathrm{R}^{4}$ & $\mathrm{R}^{5}$ & $\mathrm{R}^{6}$ & $\mathrm{R}^{7}$ & $\mathrm{X}$ & Y & Z \\
\hline $9 \mathbf{a}$ & $\begin{array}{l}\text { Calcium } \\
\text { Green-1 }\end{array}$ & 0.19 & $\mathrm{H}$ & $\mathrm{H}$ & $\mathrm{COO}^{-}$ & $\mathrm{Cl}$ & - & - & $\mathrm{H}$ & $\mathrm{O}^{-}$ & $\mathrm{O}$ & $\mathrm{CO}$ \\
\hline $9 b$ & $\begin{array}{l}\text { Calcium } \\
\text { Green-5N }\end{array}$ & 14 & $\mathrm{H}$ & $\mathrm{NO}_{2}$ & $\mathrm{COO}^{-}$ & $\mathrm{Cl}$ & - & - & $\mathrm{H}$ & $\mathrm{O}^{-}$ & $\mathrm{O}$ & $\mathrm{CO}$ \\
\hline $9 \mathrm{c}$ & $\begin{array}{c}\text { Oregon } \\
\text { Green } 488 \\
\text { BAPTA-1 }\end{array}$ & 0.17 & $\mathrm{H}$ & $\mathrm{H}$ & $\mathrm{COO}^{-}$ & $\mathrm{F}$ & - & - & $\mathrm{H}$ & $\mathrm{O}^{-}$ & $\mathrm{O}$ & $\mathrm{CO}$ \\
\hline 9d & $\begin{array}{c}\text { Oregon } \\
\text { Green } 488 \\
\text { BAPTA- } \\
\text { 6F }\end{array}$ & 3 & $\mathrm{~F}$ & $\mathrm{H}$ & $\mathrm{COO}^{-}$ & $\mathrm{F}$ & - & - & $\mathrm{H}$ & $\mathrm{O}^{-}$ & $\mathrm{O}$ & $\mathrm{CO}$ \\
\hline $9 e$ & $\begin{array}{c}\text { Oregon } \\
\text { Green } 488 \\
\text { BAPTA- } \\
5 \mathrm{~N}\end{array}$ & 20 & $\mathrm{H}$ & $\mathrm{NO}_{2}$ & $\mathrm{COO}^{-}$ & $\mathrm{F}$ & - & - & $\mathrm{H}$ & $\mathrm{O}^{-}$ & $\mathrm{O}$ & $\mathrm{CO}$ \\
\hline $9 f$ & Calcium & - & $\mathrm{H}$ & $\mathrm{H}$ & $\mathrm{COO}^{-}$ & $\mathrm{H}$ & $\mathrm{Me}$ & $\mathrm{Me}$ & $\mathrm{H}$ & $\mathrm{N}$ & ${ }^{+} \mathrm{N}$ & NHCS \\
\hline
\end{tabular}


Orange

$9 \begin{aligned} & 90 \\ & \begin{array}{l}\text { Calcium } \\ \text { Crimson }\end{array}\end{aligned}$

[a] Absorption and emission maxima for the free indicators and their complexes (No.: $\lambda_{a b s}^{\max } / \lambda_{f l}^{\max }-\lambda_{a b s}^{\max }($ complex $) / \lambda_{f l}^{\max }($ complex $)$, all values of wavelengths in nm); 9a: $506 / 531-$

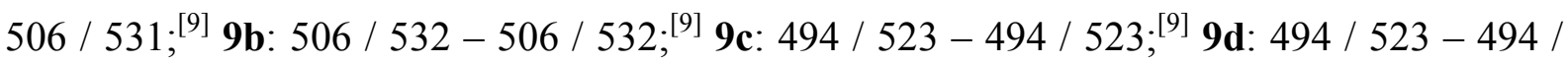
523; ${ }^{[9]} 9$ e: 494 / 521 - 494 / 521; ${ }^{[9]}$ 9f: 549 / 575 - 549 / 576; [9] $^{[9 g: ~} 583$ / $602-583$ / 602; ${ }^{[9]}$ 9h: 506 / $526-506 / 526 .^{[58]}$

Calcium Green $\mathrm{C}_{18}$ is located on the outer side of the cell membrane and may be excited with light of $500 \mathrm{~nm}$. For the first time it was prepared on the basis of the structure $9 \mathbf{a}^{[58]}$ The main disadvantage of this sensor is its very low dissociation constant $0.23 \mu \mathrm{M}$ ). This value causes determination of the near-membrane calcium spikes to be inaccurate.

The typical PET sensors 10a-c (see Section 2.1 for a description of this effect) were designed and synthesized by Prasanna de Silva and co-workers. ${ }^{[59]}$ In this series of compounds, the anthracene and pyrazoline fluorophores were separated from the conjugated system of a chelator by an $\mathrm{sp}^{3}$-carbon atom (Figure 15).<smiles>[X]c1cc(OCCOc2cc(C)ccc2N(CC(=O)[O-])CC(=O)[O-])c(N(CC(=O)[O-])CC(=O)[O-])cc1[X]</smiles>

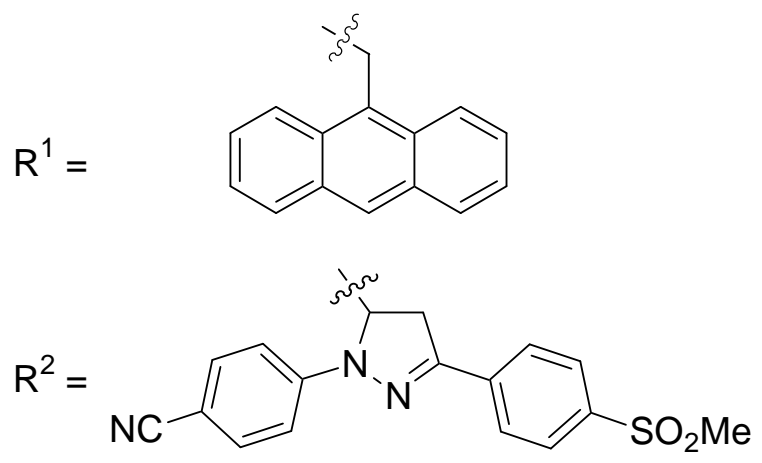

10a: QCa1: $X=\mathrm{R}^{1}, Y=H$; 10b: QCa2: $X=R^{2}, Y=H$; 10c: $X=H, Y=R^{3}$ $\mathrm{R}^{3}=$<smiles>CCOCc1c2ccccc2cc2ccccc12</smiles>

Figure 15. Highly sensitive BAPTA indicators with different fluorophores. ${ }^{[59,60]}$

Such structural features resulted in a dramatic increase in the fluorescence quantum yield upon calcium coordination; this effect was especially pronounced for compound $\mathbf{1 0 b}\left(\Phi_{\mathrm{Ca}}{ }^{2+}\right.$. 
bound $/ \Phi_{\mathrm{Ca}}{ }^{2+}$-free $=92$; and only 16 for 10a). Most of the properties of these indicators were brilliant: high extinction coefficients $\left(\varepsilon \sim 10^{4}\right)$, high selectivity for calcium in the presence of magnesium, and independence of the fluorescence intensity from $\mathrm{pH}(7.0 \leq \mathrm{pH} \leq 7.4)$. However, apart from these advantages, these sensors (10a,b) have serious drawbacks, preventing their use in biological measurements: 1) short wavelengths of the excitation and emission maxima (10a: 368 / $440 \mathrm{~nm}, \mathbf{1 0 b}: 388$ / $489 \mathrm{~nm})$; 2) low $\Phi_{f l}$-values $(\leq 0.10$; also for calcium complexes). Interestingly, the indicator 10c (Figure 15) lacked the advantages, which QCa1 had. The main reason is the presence of a second deexcitation channel due to the ether oxygen atom. Thus, the fluorescence enhancement in this case $\left(\Phi_{\mathrm{Ca}}{ }^{2+}\right.$-bound $/ \Phi_{\mathrm{Ca}}{ }^{2+}$-free $)$ is only $80 \%{ }^{[60]}$

11a: QCa3: $X=\mathrm{R}^{1}$;

11b: QCa4: $X=\mathrm{R}^{2}$;

11c: QCa5: $X=R^{3}$;

11d: Calcium Green-2: $X=\mathrm{R}^{4}, \mathrm{Z}=\mathrm{Cl}$;

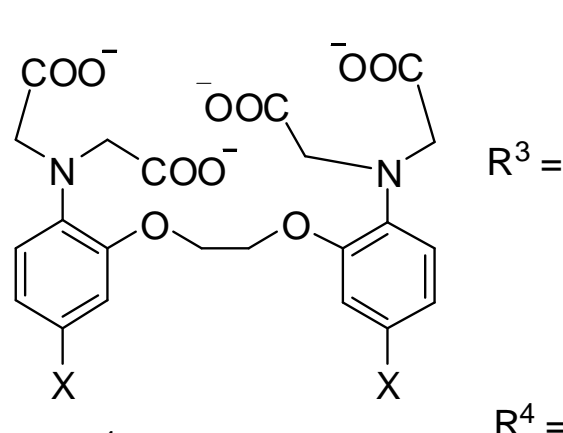

11e: Oregon Green 488 BAPTA-2: $X=R^{4}, Z=F$.<smiles></smiles><smiles>[R]=C=[W]</smiles><smiles>CN(C)c1ccc2c(C(C)(C)C)c3ccc(=[N+](C)C)cc-3oc2c1</smiles>

$\mathrm{R}^{4}=$<smiles>[X]c1cc2oc3cc(=O)c([Z])cc-3c(-c3ccc(C(N)=O)cc3C(=O)[O-])c2cc1[Z]</smiles>

Figure 16. Examples of BAPTA-sensors with two fluorophore fragments. ${ }^{[9,61]}$

With the two fluorophore fragments in the new BAPTA-based indicators presented in Figure 16, a unique fluorescence enhancement upon calcium complexation of up to 180-fold was observed. ${ }^{[9,61]}$ Unfortunately, the enormous sensitivity to calcium ions was achieved at the expense of a decrease in the fluorescence quantum yields (compounds 11a-c; both in the Ca-free and in the Ca-bound forms, compared with the monofluorophore indicators 10a, 10b, 6b). A second characteristic feature of the new indicators was associated with the lower affinities to calcium $\left(K_{\mathrm{D}}(\mathbf{1 1} \mathbf{b}) / K_{\mathrm{D}}(\mathbf{1 0 b})-20\right.$ times, $K_{\mathrm{D}}(\mathbf{1 1} \mathbf{c}) / K_{\mathrm{D}}(\mathbf{6 b})-5.5, K_{\mathrm{D}}(\mathbf{1 1 d}) / K_{\mathrm{D}}(\mathbf{9 a})-$ 2.9, $K_{\mathrm{D}}(\mathbf{1 1 e}) / K_{\mathrm{D}}(\mathbf{9 c})$ - 3.4). Most probably, these lower affinities are due to the steric hindrance caused by the two fluorophores, which destabilized the Ca-bound form relative to the Ca-free form. However, the sensor 11a with an anthracenyl-methyl fragment, which has 
more degrees of rotational freedom, showed the opposite change in its dissociation constant (1.6-fold decrease compared to 10a).

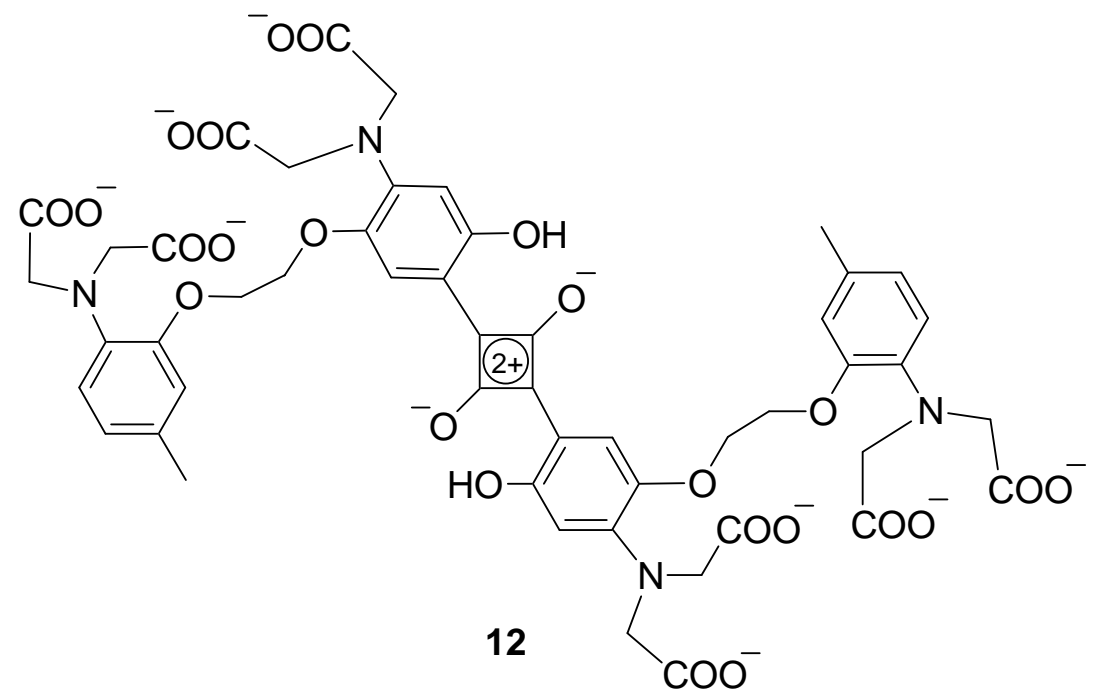

Figure 17. Calcium sensor with a squaraine fluorophore. ${ }^{[62]}$

To develop an analytical tool for calcium concentration measurements using the near-IR region, the squaraine chromophore was chosen. ${ }^{[62]}$ Its very high extinction coefficient $\left(>3 \times 10^{5}\right.$ $\left.\mathrm{cm}^{-1} \mathrm{M}^{-1}\right)$, absorption in the red region (635-690 nm) and its near-IR emission (650-700 nm) are in accordance with the requirements. Combination of this fluorophore with the BAPTA chelator gave the new calcium sensor 12. This novel indicator had its absorption maximum in the red, and the fluorescence maximum in the near-IR region $(698 / 733 \mathrm{~nm})$. Complexation with calcium is accompanied by a 6-fold decrease of the extinction coefficient and a 5-fold decrease in the emission intensity. The sensitivity to calcium of compound $\mathbf{1 2}$ is in a micromolar region.

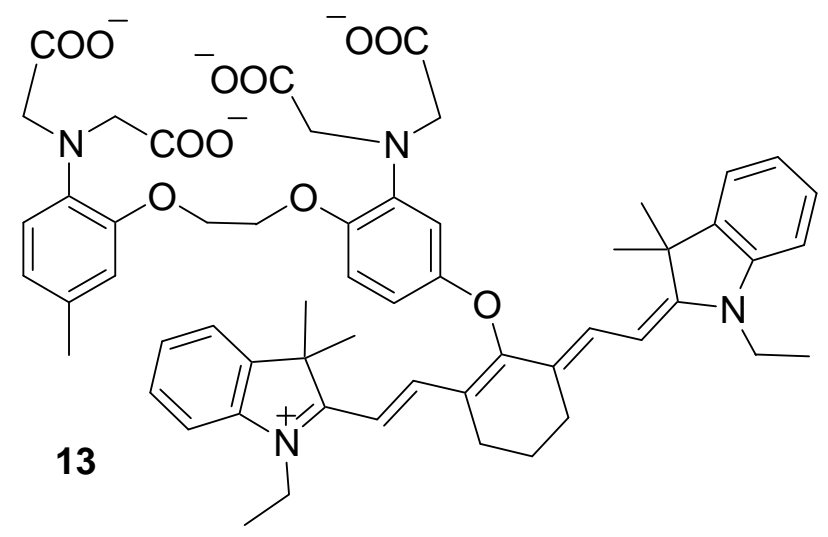

Figure 18. Calcium sensor with a cyanine chromophore. ${ }^{[63]}$ 
As a continuation of the previous work, an indicator based on a trimethylindolenine derivative of a cyanine dye was prepared. ${ }^{[63]}$ Seven methine carbon atoms between the heterocyclic fragments in $\mathbf{1 3}$ provided the required IR excitation and emission maxima (766/782 nm). Therefore, no background fluorescence was observed by using this sensor in living cells. However, with its excellent extinction coefficient $\left(2 \times 10^{5} \mathrm{~cm}^{-1} \mathrm{M}^{-1}\right)$, compound $\mathbf{1 3}$ was only a satisfactory sensor. Namely, the dissociation constant was found to be $240 \mathrm{nM}$, and the increase in the fluorescence quantum yield upon calcium coordination was found to be 2.4-fold. Although the above-mentioned parameters were in the same range as for Fura-2 and Indo-1, compound 13 was deprived of the ratiometric properties and possessed a narrow dynamic range. Low changes in the quantum yield (e. g. from 0.05 to 0.12 ) for the PET sensor $\mathbf{1 3}$ could be explained in the same manner as for the above mentioned compound 10c.

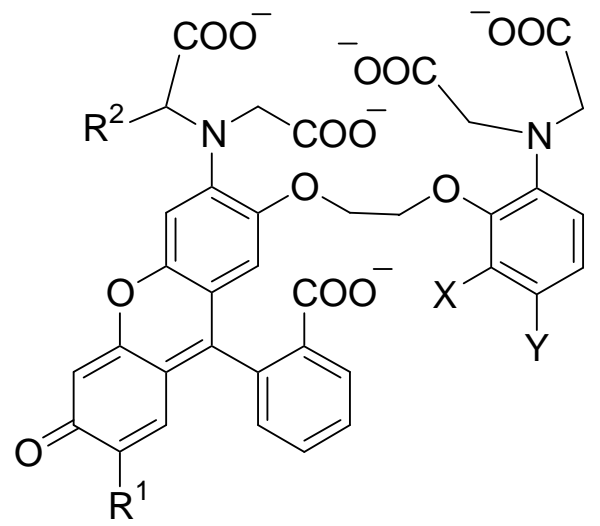

14a: $R^{1}=R^{2}=X=H, Y=M e ;$

14b: $\mathrm{R}^{1}=\mathrm{CH}_{2} \mathrm{CH}_{2} \mathrm{COOH}, \mathrm{R}^{2}=\mathrm{X}=\mathrm{H}, \mathrm{Y}=\mathrm{Me}$;

14c: FluoRhod-2: $\mathrm{R}^{1}=\mathrm{CH}_{2} \mathrm{CH}_{2} \mathrm{COOH}, \mathrm{R}^{2}=\mathrm{Y}=\mathrm{Me}, \mathrm{X}=\mathrm{H}$;

14d: Fluo-Rhod-F: $\mathrm{R}^{1}=\mathrm{CH}_{2} \mathrm{CH}_{2} \mathrm{COOH}, \mathrm{R}^{2}=\mathrm{H}, \mathrm{X}=\mathrm{Y}=\mathrm{F}$.

Figure 19. The FluoRhod series of indicators. ${ }^{[64]}$

The mode of incorporating of the BAPTA chelator into excellent and bright fluorophores, such as fluoresceins and rhodamines, was firstly described by R. Tsien in $1989,{ }^{[44]}$ but it was later rejected by him as unpromising. However, this approach was implemented several years later by G. Smith in the double-ratiometric sensor FluoRhod-2 (see Figure 19). ${ }^{[64]}$ As it concerns fluorescence quantum yields and the spectral shift upon calcium coordination, the new indicator was like Fura-2. However, as it was predicted by R. Tsien, compound 14a had a higher dissociation constant and, due to the rhodole structure as a fluorophore, the absolute value of the fluorescence quantum yield was lower than for the parent xanthenes (fluorescein and the rhodamines). Undesirable features, like a decrease in the calcium affinity and a low 
$\mathrm{pK}_{\mathrm{a}}$-value of the initial indicator 14a (which produces a dependence of the fluorescence ratios of an indicator at $\mathrm{pH}$-values even in the physiological range) were corrected later by variation of substituents. Thus, introduction of a carboxyethyl fragment into the $\mathrm{R}^{1}$ position (see Figure 19, intermediate structure $14 \mathrm{~b}$ ) provided the required $\mathrm{pK}_{\mathrm{a}}$ value of 6.7 and therefore, independence of the fluorescence from $\mathrm{pH}$ in the physiological range of 7.0-7.4. Introduction of an additional methyl group into one of four carboxymethyl residues enabled one to decrease the $K_{\mathrm{D}}$ value from $10 \mu \mathrm{M}$ to $1 \mu \mathrm{M}$ (see Figure 19, final structure $14 \mathrm{c}$ ). Moreover, compound 14c, in comparison with the most popular ratiometric indicators (Fura-2, Indo-1), had suitable shifts of both spectral maxima upon calcium complexation (absorption: $57 \mathrm{~nm}$; emission: $30 \mathrm{~nm}$ ). An analogue of 14c was prepared, which got the name Fluo-Rhod-F and could potentially be applied in dual measurement modes: both in ${ }^{19} \mathrm{~F}$ NMR spectroscopy and fluorescence. The presence of two fluorine atoms in compound $\mathbf{1 4 d}$ resulted in a lower calcium affinity $\left(K_{\mathrm{D}}>10 \mu \mathrm{M}\right)$, compared to the related compound $\mathbf{1 4 c} .^{[39 b]}$

\subsection{APTRA and its derivatives}

Replacing one imino diacetic fragment in the EDTA chelator (see Figure 6) with an oxyacetic fragment, together with an ethylene bridge containing an aromatic ring, gave the APTRA chelator (15a). It has a reduced number of coordinating atoms (5 vs. 6) and a reduced number of nitrogen coordination sites (in favor of oxygen atoms). Moreover, the APTRA chelator contains significantly less coordinating heteroatoms than the BAPTA chelator described in section 2.4 (5 vs. 8). 

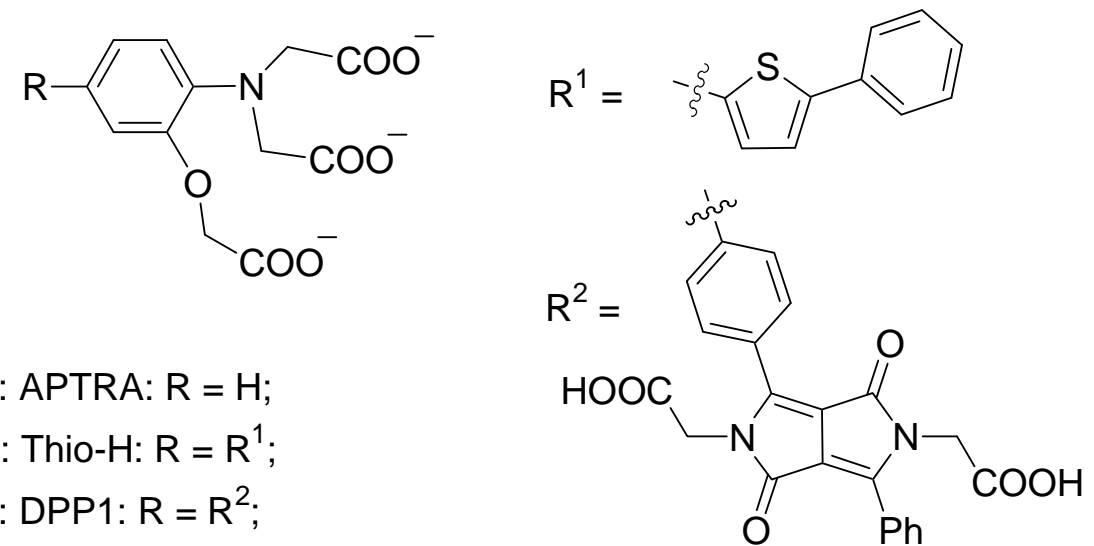

15a: APTRA: $\mathrm{R}=\mathrm{H}$;

15b: Thio-H: $\mathrm{R}=\mathrm{R}^{1}$;

15c: $D P P 1: R=R^{2}$;

15d: $R=R^{3}, X=M e ;$

15e: $\mathrm{R}=\mathrm{R}^{3}, X=4-\mathrm{MeOC}_{6} \mathrm{H}_{4}$;

15f: $R=R^{4}, Y=P h$;

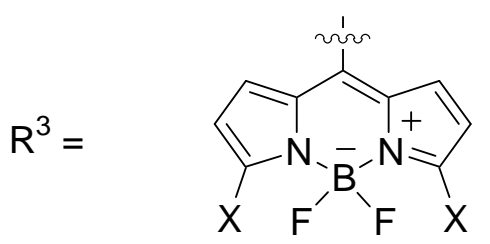

15g: $\mathrm{R}=\mathrm{R}^{4}, \mathrm{Y}=4-\mathrm{MeOC}_{6} \mathrm{H}_{4}$;

15h: $\mathrm{R}=\mathrm{R}^{4}, \mathrm{Y}=4-\mathrm{FC}_{6} \mathrm{H}_{4}$;

15i: $\mathrm{R}=\mathrm{R}^{4}, \mathrm{Y}=4-\mathrm{O}_{2} \mathrm{NC}_{6} \mathrm{H}_{4}$;

15j: $R=R^{4}, Y=5-O_{2}$ Nthienyl.

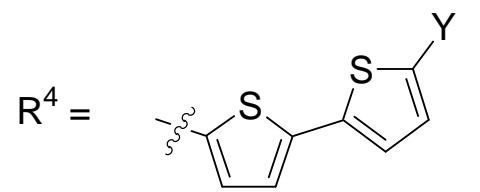

Figure 20. A variety of APTRA-based calcium indicators. ${ }^{[65-68]}$

Obviously, a change in quality, quantity and spatial localization of chelator ligands influences the coordination properties of the indicator. Thus, for example, compound $\mathbf{1 5} \mathbf{b}^{[65]}$ had a dissociation constant of the calcium complex in the range of $50 \mu \mathrm{M}$ and the $\Phi_{f l}$-value for the Ca-free ligand 0.74 . In principal, such values could be perfect for indicators, but only in terms of the absolute values. More important are the changes in these values upon calcium coordination; and these were not so promising for Thio-H (15b): a) the decrease in fluorescence quantum yield was only 1.5 fold (not perfect for an application of this indicator in fluorescence mode); b) compound $\mathbf{1 5 b}$ was 100 times less selective for $\mathrm{Mg}^{2+}$ than Fura-2 (having the same $K_{\mathrm{D}}$ for $\mathrm{Mg}^{2+}$ as Fura-2, compound 15b has a $K_{\mathrm{D}}$ for $\mathrm{Ca}^{2+}$ which is in 100 times higher than that of Fura-2); c) an undesirable sensitivity of Thio-H to temperature and $\mathrm{pH}$ in the physiological range (7.0-7.4) was observed; d) like Fura-2 (5a), it has to be excited with blue or UV light ( $\lambda_{a b s}^{\max }=351 \mathrm{~nm}$ ); e) upon calcium coordination, this indicator displays rather small shifts in its absorption (ca. $19 \mathrm{~nm}$ ) and emission spectra (no shift), which prohibits ratiometric measurements. All the factors mentioned above cause compound $\mathbf{1 5 b}$ not to be very promising for applications in biology.

In order to develop more suitable indicators, further efforts were aimed at the replacement of the substituents in the para-positions relative to the amino group. The second candidate 
15c had better properties as a biocalcium indicator: a) absorption and emission maxima were in the visible region $(470 / 538 \mathrm{~nm}) ; \mathrm{b})$ increase in the fluorescence quantum yield upon calcium coordination was in the same range as for Quin-2 $\left(\Phi_{\mathrm{Ca}}{ }^{2+}\right.$-bound $(0.048) / \Phi_{\mathrm{Ca}}{ }^{2+}$-free $(0.012)=4)$, although the absolute values were not very high; c) the dissociation constant $\left(K_{\mathrm{D}}=10 \mu \mathrm{M}\right)$ was suitable for detection of elevated intracellular levels of calcium. ${ }^{[66]}$

Two PET calcium indicators $\mathbf{1 5 d - e}$ were obtained by a favorable combination of an APTRA chelator with BODIPY ${ }^{\circledR}$ derivatives. ${ }^{[67]}$ Both indicators had photophysical properties very similar to those of Fluo-3, i.e. fluorescence quantum yields and increase in fluorescence intensities were actually the same $\left(\mathbf{1 5 d}\right.$ : $\Phi_{\mathrm{Ca}}{ }^{2+}$-bound $=0.14 / \Phi_{\mathrm{Ca}}{ }^{2+}{ }_{\text {-free }}=0.004$, $I_{C a^{2+}-\text { free }}^{f l} / I_{C a^{2+} \text { bound }}^{f l}=40$; 15e: $\left.\Phi_{\mathrm{Ca}}{ }^{2+}{ }^{2+}{ }_{\text {bound }}=0.13 / \Phi_{\mathrm{Ca}}{ }^{2+}{ }_{- \text {free }}=0.003, I_{\mathrm{Ca}^{2+}-\text { free }}^{f l} / I_{\mathrm{Ca}^{2+} \text {-bound }}^{f l}=30\right)$. The compound 15d even had virtually the same excitation and emission maxima (505/525 $\mathrm{nm})$ as Fluo-3. The second indicator 15e with two $p$-methoxyphenyl substituents, showed wavelength maxima shifted to the red $(570 / 625 \mathrm{~nm})$. A red shift in the spectra favors practical applications. The main difference to "Tsien's sensor" (Fluo-3) was the new "working interval" (which has to be from $0.1 K_{\mathrm{D}}$ to $10 K_{\mathrm{D}}$ ): the dissociation constants of $\mathbf{1 5 d}$,e were $100 \mu \mathrm{M}$, i.e. in 250 times greater than that of Fluo-3.

Other derivatives of $\mathbf{1 5 b}$ were prepared with an additional thiophene ring and with functional groups in conjugation with the amino group (see Figure 20). ${ }^{[68]}$ Extension of the conjugation path by the additional thiophene ring gave compound $\mathbf{1 5 f}$ with a red-shifted excitation maximum (35 $\mathrm{nm}$ in comparison with Thio-H). However, the same extension of the conjugated system could be the main reason for the decrease in the absolute value of the fluorescence quantum yield (to 0.25 ). The latter value was successfully compensated by the 4fold fluorescence modulation upon calcium complexation (which indicates a higher sensitivity of compound $\mathbf{1 5 f}$ to calcium in comparison with the sensitivity of indicator $\mathbf{1 5 b}$ ). The introduction of a donor methoxy group improved the properties of indicator 15g: the reduction of $\Phi_{f l}$ observed by a transition from the Ca-free form to the Ca-bound form was found to be a 13-fold already. The incorporation of electron acceptor groups in compound $\mathbf{1 5 f}$ produced a significant decrease in the $\Phi_{f l}$ values of the free and bound form of indicators (for the fluorine substituent by a factor of two and for a nitro group by a factor of 500), that made the fluorescence signal of indicators $\mathbf{1 5 h}$ and $\mathbf{1 5 i}$ hard to detect. The replacement of the phenyl fragment with a thienyl ring in $\mathbf{1 5} \mathbf{i}$ resulted in a compound $\mathbf{1 5} \mathbf{j}$ without any detectable changes in the fluorescence signal upon calcium coordination. It was possible to apply 
indicators $\mathbf{1 5 f}-\mathbf{j}$ in dual ratiometric modes, because these compounds had a pronounced shift in both spectral maxima; the most promising were compounds $\mathbf{1 5 f}-\mathbf{g}$.

\subsection{Crown-based calcium indicators}

Like in the case of the charged chelators (BAPTA), the search for calcium sensors with crown-ether residues started from compounds analytical chemistry. ${ }^{[69]}$ Interestingly, the same connecting mode was used to bind the ionophore to the fluorophore. As in the case of Calcein, a Mannich reaction was applied for this purpose to provide compound $\mathbf{1 6 .}$

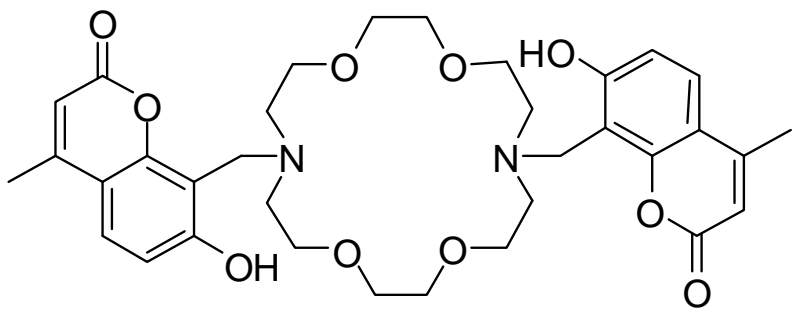

16

Figure 21. Fluorimetric crown ether reagent for calcium detection. ${ }^{[69]}$

This sensor 16 showed a large shift in the UV band upon calcium complexation (from 326 to $380 \mathrm{~nm}$ ). It was successfully used for the so-called "extraction-fluorimetric" method of calcium determination. This method involves the extraction of calcium from an aqueous medium with compound $\mathbf{1 6}$ to an organic solvent followed by measurements of the fluorescence signal of the organic solution. With this method, it is possible to assess the content of calcium on a ppb level.

Due to the success of the BAPTA-sensors, the development of the crown-containing Casensors progressed fairly slowly. All known crown-containing sensors were designed for and studied in non-aqueous solutions (usually in acetonitrile). Most of the compounds of this class were not described as indicators, but as host compounds with some interesting sensing properties.

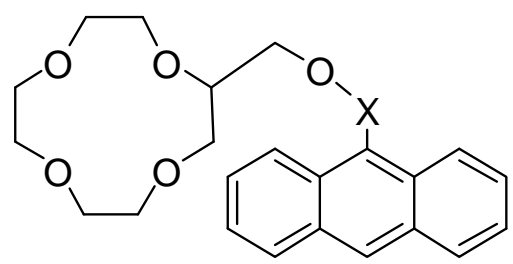

17a: $\mathrm{X}=\mathrm{CO}$;

17b: $X=\mathrm{CH}_{2}$. 
Figure 22. Calcium indicators with a 12 -crown- 4 as a coordinating moiety. ${ }^{[70]}$

Only two calcium-sensing hosts with a 12-crown-4 ether as the main coordinating element has been reported. ${ }^{[70]}$ The two variants $\mathbf{1 7} \mathbf{a}-\mathbf{b}$, absorbing in the UV region (at about $360 \mathrm{~nm}$ ) were selectively sensitive to calcium, but not to other alkali and alkali earth metal cations. However, these compounds had relatively low fluorescence quantum yields $(0.26$ and 0.10, respectively). They have different fluorescence maxima (17a: $460 \mathrm{~nm}, \mathbf{1 7 b}: 412 \mathrm{~nm})$, and opposite directions of the spectral changes upon calcium coordination: the first anthracene containing "host" 17a displayed a two-fold decrease in the fluorescence signal, while the second $\mathbf{1 7 b}$ produced a two-fold increase.

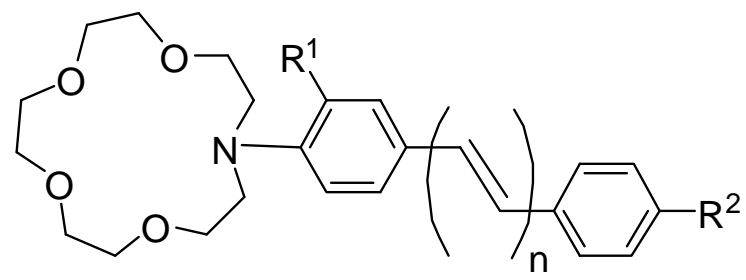

18a: DS-crown: $R^{1}=R^{2}=H, n=1$;

18b: DCS-crown: $R^{1}=H, n=1, R^{2}=C N$;

18c: PDS-crown: $R^{1}=H, n=1, R^{2}=P h$;

18d: DDB-crown: $R^{1}=H, n=2, R^{2}=N_{2 M e}$;

18e: DDS-crown: $R^{1}=H, n=1, R^{2}=N^{2} e_{2}$;

18f: DDCS-crown: $R^{1}=C N, n=1, R^{2}=N^{2} e_{2}$.

Figure 23. Stilbene-based calcium indicators 18a-f. ${ }^{[71,72]}$

An attempt to use stilbene derivatives as potential fluorophores for calcium sensing was reported as early as 1985 by Tsien ${ }^{[35]}$ However, because of the low fluorescence quantum yields and the photochemical side reactions, stilbene indicators were abandoned. Nevertheless, eight years later another research group investigated the photophysical properties of stilbene-containing dyes. In this study, it was discovered that the crown-ether containing stilbene 18a produced a $20 \mathrm{~nm}$ blue shift of the emission maximum upon calcium complexation. ${ }^{[71 \mathrm{a}]}$

Further research on this stilbene derivative and its analogues with different substituents resulted in two PCT sensors $\mathbf{1 8 b}-\mathbf{c}$ with high sensitivity to calcium. ${ }^{[71 b]}$ The crown-containing "push-pull" compound $\mathbf{1 8 b}$ and biphenyl compound $18 \mathrm{c}$ have more attractive properties than the only donor-substituted compound 18a. For example, the latter is poorly fluorescent $\left(\Phi_{f l}=\right.$ $1.3 \%$ in acetonitrile), while these $\Phi_{f l}$ values for compounds $\mathbf{1 8 b}$ and $\mathbf{1 8 c}$ were found to be 6 and 27 times higher, respectively. In addition, compounds $\mathbf{1 8 b}-\mathbf{c}$ display a $50 \mathrm{~nm}$ blue shift in 
absorption maxima upon calcium coordination. However, their main absorption bands are in the UV region: $\lambda_{a b s}^{\max }=385 \mathrm{~nm}$ for the free ligand $\mathbf{1 8 b}$ in $\mathrm{MeCN}, \lambda_{a b s}^{\max }=374 \mathrm{~nm}$ for the free ligand 18c in BuCN. Unfortunately, neither of these compounds showed sufficient selectivity for calcium in the presence of barium (e.g. $\ln K_{\mathrm{s}}\left(\mathrm{Ca}^{2+}\right)=4.20$ and $\ln K_{\mathrm{S}}\left(\mathrm{Ba}^{2+}\right)=3.74$ for 18b). Besides that, a 2.2-fold decrease in the fluorescence quantum yield was found for $\mathbf{1 8 c}$ upon calcium coordination, which is not optimal for a ratiometric probe.

The introduction of two donor groups into the stilbene core radically changed the spectroscopic properties of the "push-pull" sensors 18d-f (see Figure 23). ${ }^{[72]}$ Upon calcium complexation, the azacrown moiety with a calcium cation inside acts as an acceptor, which causes a strong intramolecular charge transfer (ICT) from the dimethylamino group to the crown ether upon excitation with light. As a consequence of the ICT, a significant red shift in the fluorescence spectra results (upon complexation with calcium). The values of these shifts varied from 36 to $80 \mathrm{~nm}$ (18d-f). Surprisingly, the prolongation of the conjugation chain almost did not affect this shift ( $\Delta \lambda_{f l}^{\max }$ for compound $\mathbf{1 8 d}$ was $43 \mathrm{~nm}$ vs. $36 \mathrm{~nm}$ for compound 18e). However, the introduction of a nitrile acceptor group into the ortho-position to azacrown residue caused a two-fold increase of the shift, owing to the higher stabilization of the state with a negative charge on the aromatic ring. Such favorable changes in the fluorescence spectra upon calcium coordination enable one to use these substances in ratiometric measurements. Unfortunately, the absorption spectra demonstrated only small blue (18e: $7 \mathrm{~nm}, \mathbf{1 8 d}$ : $8 \mathrm{~nm}$ ) or red ("unproductive") shifts (18f: $2 \mathrm{~nm})$. As candidates for calcium sensors, the stilbene derivatives have important drawbacks: a decrease in the fluorescence quantum yield for the calcium complexes from 2.1 times for compound $\mathbf{1 8 f}$ to 10 times for 18e, and the necessity of UV excitation (at $359-385 \mathrm{~nm}$, for the free ligands and their complexes).
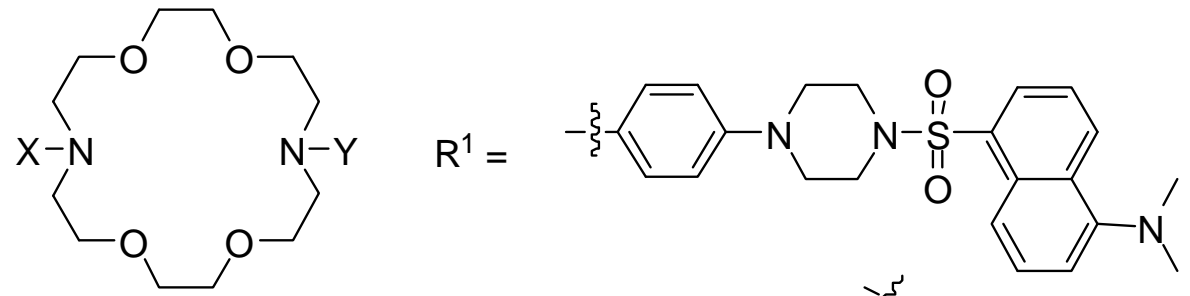

19a: $X=\mathrm{CH}_{2} \mathrm{Ph}, \mathrm{Y}=\mathrm{R}^{1}$;

19b: $X=P h, Y=R^{2}$;

19c: $\mathrm{X}=p$-chlorophenyl, $\mathrm{Y}=\mathrm{R}^{2}$;

19d: $X=m$-chlorophenyl, $Y=R^{2}$;

19e: $X=R^{3}, Y=R^{2}$.

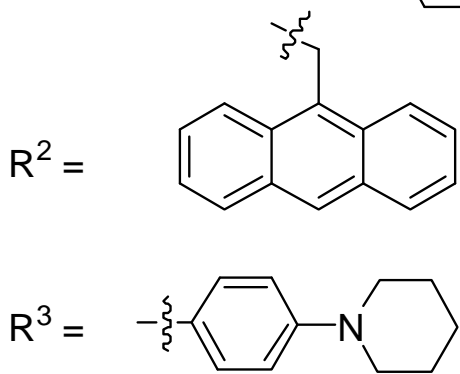


Figure 24. Calcium-sensitive fluorescent molecules with diaza-18-crown-6 chelation moieties. ${ }^{[73 a, 80]}$

A. Pearson and co-workers showed that diaza-18-crown-6 ether derivatives (Figure 24) are also applicable for calcium sensing. ${ }^{[73 a]}$ By exploration of the PET effect in the series of dansyl amides (e. g. compound 19a in Figure 24), a new highly selective calcium indicator compound 19a - was discovered. In this compound, the dansyl group plays the role of an electron acceptor, while the tetraalkyl- $p$-phenylenediamine (TAPD) residue with an incorporated crown ring is a strong electron donor. The sensor 19a could be used for measurements exclusively in the fluorescence mode. It produces a strong increase in the fluorescence intensity upon complexation with calcium -5.5 times (at $\lambda_{f l}^{\max }=540 \mathrm{~nm}$ ). The ratiometric mode in this case does not work, because compound 19a provides only an $8 \mathrm{~nm}$ hypsochromic shift of the absorption maximum in the UV region (after complexation). It should be noted that 19a has a low affinity for calcium $\left(\log K_{\mathrm{s}}=3.2\right)$, and a low value of the fluorescence quantum yield (0.04).

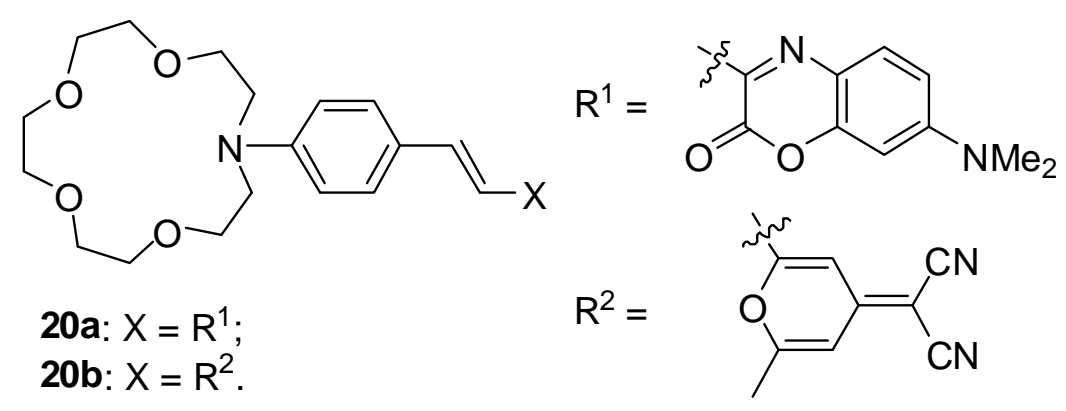

Figure 25. Calcium sensitive fluorescent molecules with aza-15-crown-5 chelation moieties. $^{[74,75]}$

The first rational synthesis of a crown-based fluorescent calcium sensor, that of 20a (Figure 25) was carried out by B. Valeur and co-workers in $1988 .{ }^{[74]}$ This styrene-containing indicator with a benzoxazinone fluorophore displayed a $20 \mathrm{~nm}$ hypsochromic shift in the absorbtion spectrum upon coordination with calcium and, as typical for a PCT sensor, a large $(68 \mathrm{~nm})$ blue shift (from 642 to $574 \mathrm{~nm}$ ) of the fluorescence maximum. Besides that, metal complexation increased the fluorescence quantum yield (e. g. for $\mathrm{Ca}^{2+}: \Phi_{\text {bound }}(0.64) / \Phi_{\text {free }}$ $(0.33)=1.9)$. It may be insufficient for the direct fluorimetric measurements, but can be a good addition for the ratiometric properties. Compound 20a had an insufficient selectivity for calcium in the presence of barium $\left(\log K_{\mathrm{s}}\left(\mathrm{Ca}^{2+}\right)=4.14 ; \log K_{\mathrm{s}}\left(\mathrm{Ba}^{2+}\right)=3.62\right)$. However, 
barium does not occur in living tissues in any significant amounts, and therefore compound 20a may be used as a $\mathrm{Ca}^{2+}$ sensor there.

The replacement of the donor-acceptor type benzoxazinone chromophore with an acceptor - dicyanomethylene-4H-pyran - caused some drastic "mirror" changes in the photophysical behavior of respective indicator. ${ }^{[75]}$ The new sensor $20 \mathbf{b}$ shows a strong $66 \mathrm{~nm}$ blue shift in its absorption spectrum upon calcium coordination. This shift, of course, makes ratiometric measurements possible. Upon increase in metal ion concentration, the fluorescence maximum in the spectrum of compound 20b was only slightly shifted, and this was accompanied by a steep decrease of the fluorescence quantum yield (about 3 fold). As compared to 20 a, the stability constants of the new indicator did not change significantly (log $\left.K_{\mathrm{s}}\left(\mathrm{Ca}^{2+}\right) \approx 3.76\right)$.

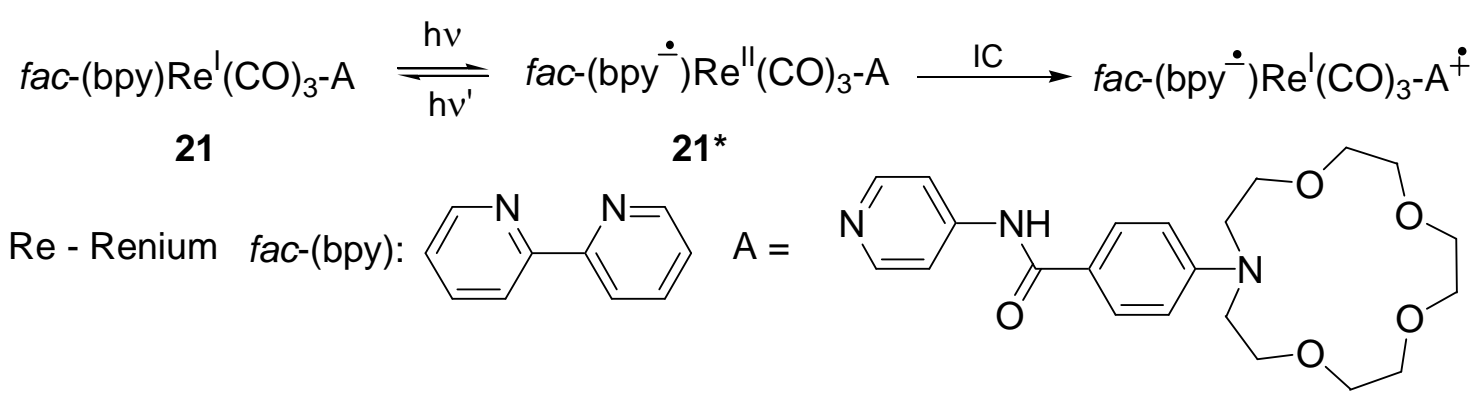

Scheme 5. The working principle of a transition metal complex indicator for calcium $21 .{ }^{[76]}$

An unusual sensor for calcium was described by McQueen et al. ${ }^{[76]}$ This indicator 21 is based on the rarely used principle that upon activation by light by means of a metal-to-ligand charge transfer (MLCT) it is transferred into the excited state $\mathbf{2 1}^{*}$. This state has two possible relaxation paths: 1) return to the ground state with emission of light of frequency $v^{\prime}$ or 2) internal conversion (IC) with electron transfer from the other ligand's donor atom to the metal. The nitrogen atom of the azacrown fragment in ligand A suites very well to provide the internal conversion. It was found that the coordination of a positively charged ion with the crown ether excludes the IC decay that results in the return of the excited molecule to the ground state with the emission of light. The highest increase in fluorescence and fluorescence quantum yield among various cations is produced by calcium (7-fold), although the absolute value of $\Phi_{f l}$ is moderate, only 0.012 , despite an excess of calcium. The position of the fluorescence maximum was found to be at $600 \mathrm{~nm}$, and remained unshifted, while the absorption maximum shifted by $45 \mathrm{~nm}$ (to $305 \mathrm{~nm}$ ) upon complexation with calcium. 


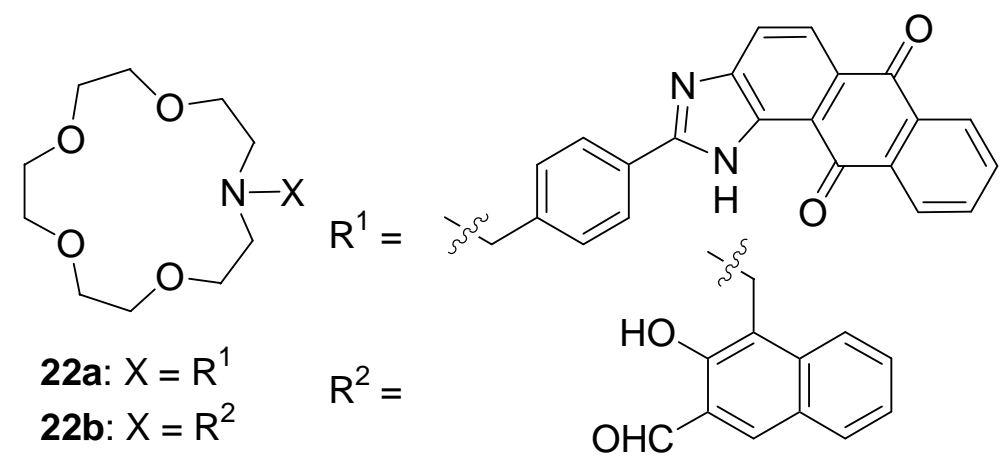

Figure 26. Calcium sensors with an aza-15-crown-5 ether moiety in a chelator role. ${ }^{[77]}$

An absolutely new fluorophore, 2-phenylimidazo[5,4-a]anthraquinone ( $\mathrm{R}^{1}$, Figure 26), emitting and absorbing in the visible region with $\lambda_{a b s}^{\max }=405 \mathrm{~nm}$ and $\lambda_{f l}^{\max }=515 \mathrm{~nm}$, was used to construct the PET chemosensor 22a ${ }^{[77]}$ This compound demonstrated a maximum value of the stability constant for its complex with $\mathrm{Ca}^{2+}\left(\log K_{\mathrm{s}}=6.51\right)$ and a maximum enhancement of the fluorescence quantum yield upon calcium coordination, in comparison with other alkali and alkaline earth cations. However, the low value of its fluorescence quantum yield $(\Phi=$ $0.7 \%$ ) and low selectivity for calcium in the presence of magnesium and barium, do not favor the application of compound 22a for calcium determination. The low selectivity is reflected in the small difference between the stability constants for $\mathrm{Mg}^{2+}, \mathrm{Ca}^{2+}, \mathrm{Ba}^{2+}$ (not higher than one logaritmic unit).<smiles>[CH]Oc1c(C=O)cc2ccccc2c1C[N+](CC)(CC)CC</smiles>

Excited state of conf. A<smiles>C=CC1CC[NH+](CC)Oc2c(C=O)cc3ccccc3c2C1</smiles>

Tautomer A<smiles>CCN(CC)Cc1cc2ccccc2cc1O</smiles>

Tautomer B<smiles></smiles>

Excited state of conf. B

Scheme 6. Graphical demonstration of the ESIPT effect as exemplified for the compound DMHN (1-[(diethylamino)methyl]-2-hydroxy-3-naphthaldehyde). ${ }^{[78 b]}$

A promising calcium sensor (compound $\mathbf{2 2 b}$, Figure 26) based on the highly fluorescent 3-hydroxynaphthalene-2-carbaldehyde was prepared. $^{[78 a}$ Its dialkylaminomethylene derivative - the famous solvatochromic compound DMHN (Scheme 6) - showed significant red shifts $(\approx 100 \mathrm{~nm})$ in the excitation and emission maxima upon increasing solvent polarity. Such spectral changes occurred due to an excited state intramolecular proton transfer (ESIPT).${ }^{[78 b]}$ Instead of the two ethyl groups, the novel indicator $\mathbf{2 2 b}$ had a crown-ether ring. 
Undoubtedly, the ESIPT effect largely influences the photochemical and photophysical

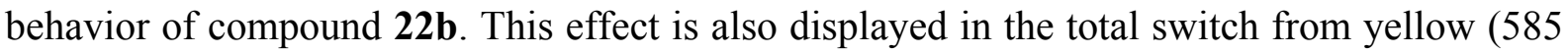
$\mathrm{nm}$ ) to the near-infrared emission at $730 \mathrm{~nm}$ in the presence of high sodium concentrations, and in a $20 \mathrm{~nm}$ shift in the emission maxima upon calcium complexation. However, the main factor that explains the changes in fluorescence upon metal-ion coordination could be PET. Obviously, the PET effect takes place in 22b, as well as in other crown sensors (like 22a, 19, 17 Figure 26, 24, 22). However, because of the presence of the acidic phenol hydroxy group in compound 22b (which decreases the efficiency of the electron transfer from amino group) the PET-effect is not very pronounced. Therefore, it becomes possible to explain the opposite directions in fluorescence changes observed upon metal-ion coordination with compound 22b. Owing to the presence of the crown ring, sodium binds with the the lone electron pair of the nitrogen atom better than a proton. However, $\mathrm{Na}^{+}$does not suppress PET as effectively as a proton. The overall result of sodium binding is a decrease in fluorescence intensity at 585 $\mathrm{nm}$. The opposite effects were observed in the case of calcium: the doubly charged calcium suppresses the PET effect better than a proton. The result of the PET-effect suppression is an increase in fluorescence intensity upon calcium complexation. Thus, having the same stability constant $\left(K_{\mathrm{s}}\left(\mathrm{Ca}^{2+}\right)=3.8 \times 10^{4} ; K_{\mathrm{s}}\left(\mathrm{Na}^{+}\right)=2.3 \times 10^{4}\right)$ and the opposite effect on fluorescence, it is possible to use compound $\mathbf{2 2} \mathbf{b}$ as a calcium sensor, though only in the absence of sodium, and with accurate $\mathrm{pH}$ control (in analogy to $\mathrm{DMHN}$, the compound $\mathbf{2 2} \mathbf{b}$ is sensitive to the $\mathrm{pH}$ value).

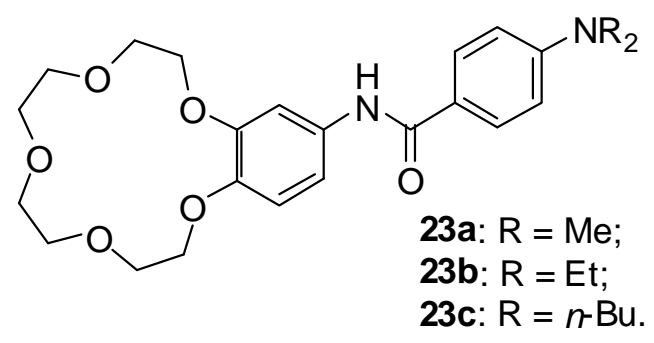

Figure 27. Calcium indicator with a $p$-aminobenzanilide fluorophore. ${ }^{[79]}$

A simple fluorophore fragment $-p$-aminobenzanilide - was utilized by Liu et al. for the creation the alkali and alkaline earth metal-ion sensors. ${ }^{[79]}$ The functional principle of these indicators is relatively simple: in the free state, compounds 23a-c have the D-A-D' sequence (D - electron donor, A - electron acceptor). At the same time, one of the terminal donor components is a 15-crown-5 ether with two oxygen atoms, participating in conjugation with the benzene ring. This sequence changes to D-A-A' upon cation complexation (including calcium), because the metal ion has high electron-withdrawing properties. Also, the presence 
of two interacting centers, the oxygen atoms attached to the aromatic ring, instead of one nitrogen atom (as it was in most of the crown sensors described above), influences the spectroscopic properties. The changes in the electron density distribution upon $\mathrm{Ca}^{2+}$ coordination were a slight red shift in the absorption maximum (from 320 to $324 \mathrm{~nm}$ for $\mathbf{2 3 b}$ ) and a dramatic increase in both fluorescence maxima (at 373 and $497 \mathrm{~nm}$ for compound 23ac) with a leap in fluorescence quantum yield (up to 22.7-fold for the complex of compound 23b with calcium). Only the short wavelength fluorescence maximum had a red shift up to 25 $\mathrm{nm}$ (for the complex of compound 23a with calcium). However, as has already been mentioned above, such an indicator is not of any interest for the area of research addressed here, i. e. cells and their components. The main features that make its applications for such purposes impossible are: a) excitation and emission bands in the UV region; b) similar stability constants for the complexes with other biologically relevant metal ions $\left(\log K_{\mathrm{s}}\left(\mathrm{Ca}^{2+}\right)\right.$ $\left.\left.=5.54 ; \log K_{\mathrm{s}}\left(\mathrm{Mg}^{2+}\right)=5.58\right) ; \mathrm{c}\right)$ extremely low absolute values of $\Phi_{\mathrm{fl}}(<1 \%)$.

In the early work of Pearson et al. ${ }^{[73]}$ some aza-18-crown- 6 ethers (only the best sensor from this series 19a is presented in Figure 24) with a suitable selectivity for calcium were reported. Later on, in combination with an anthracene residue as a fluorophore, a series of new calcium indicators were prepared (compound 19b-e, Figure 24). ${ }^{[80]}$ The simplest compound 19b is 11 times more sensitive and 5 times more selective than the BAPTA analog QCa-1. This improvement may be explained by the direct attachment of the sensitive nitrogen atom to the fluorophore (in contrast to QCa-1, in which the aromatic ring is inserted between them). Due to the presence of an anthracene fluorophore, compound 19b has the same moderate fluorescence quantum yield (0.20) as QCa-1. As regards the coordinative ability, compound $19 \mathrm{~b}$ has a stability constant $\left(K_{\mathrm{s}}\right)$ which is two orders of magnitude higher than that of the analogue 19a. Variation of the substituents at the remote nitrogen atom seriously changed the sensitivity (up to an 8-fold increase for compound 19c in comparison with 19b) and influenced the selectivity for calcium over magnesium (up to 7.3-fold decrease for 19e in comparison with 19b).

\subsection{Calixarene hosts as coordination moieties for calcium sensors}

Quite recently, calixarenes - a very special class of host compounds - became good candidates in the wide variety of new calcium sensors. 


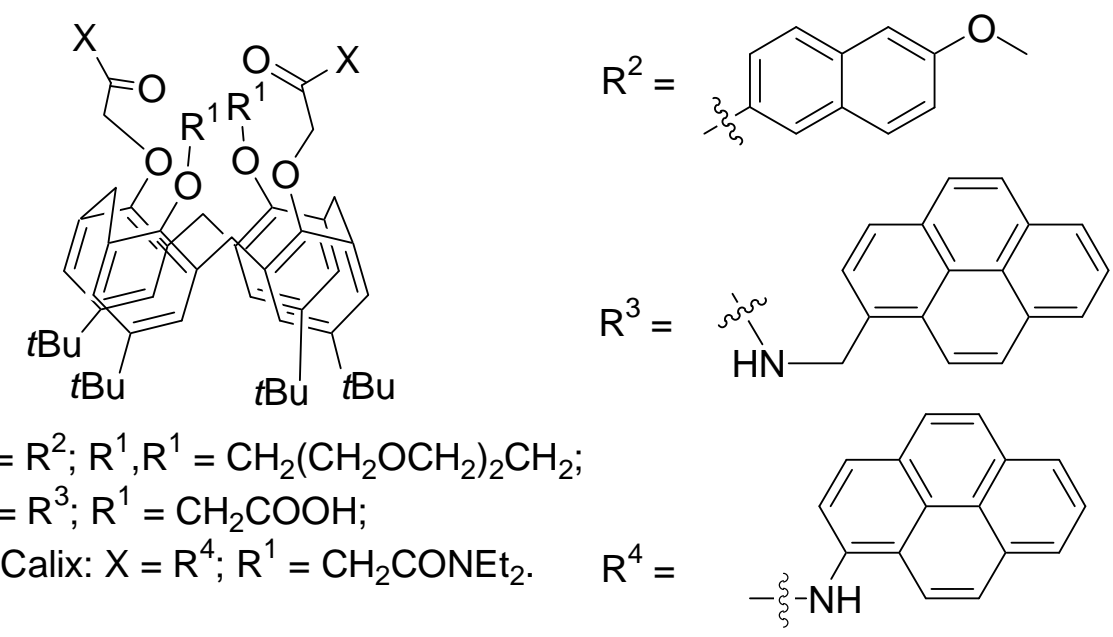

Figure 28. Calcium sensors with calix[4] arene moieties as chelators. ${ }^{[81,82]}$

Calixcrowns (e. g. compound 24a, Figure 28) - a special class of macrocyclic compounds - were prepared by incorporation of the crown ether fragments to the opposite sides of calix[4]arene. ${ }^{[81]}$ The heteroatoms of the lariat crown ether and pendant groups (in the current case carbonyl oxygens) form a three-dimensional net of binding sites, which can effectively catch cations with suitable ion radii. Calcium ions cause two types of changes in the spectroscopic parameters of compound 24a: a) a red shift in the absorption (18 nm) and emission bands $(34 \mathrm{~nm})$ owing to the typical PCT-sensor behavior upon coordination to oxygen atoms of the (acceptor) carbonyl groups; b) 4.5-fold increase in fluorescence intensity. The latter change may be explained considering that the fluorophore molecule is prevented from self-quenching of the fluorescence of the two naphthalene fragments, as they are moved apart upon coordination of 24a with calcium. Unfortunately, all studies were carried out in acetonitrile only. Besides that, the sensor 24a had an absorption maximum in the UV region $(310 \mathrm{~nm})$, and the fluorescence band was found at the edge of the visible region, i. e. at 400 $\mathrm{nm}$.

Two very similar calixarene sensors were investigated by Korean and American researchers. ${ }^{[82 a, b]}$ The first sensor - compound $\mathbf{2 4} \mathbf{b}^{[82 a]}$ - is more water-soluble (due to the presence of two carboxylic acid groups). It has an additional methylene group between the pyrene ring and the amido group. The second sensor $\mathbf{2 4 c ^ { [ 8 2 b ] }}$ has $N, N$-diethylcarboxamido groups instead of the carboxylic acid functions, and the amido fragment is directly attached to the pyrene ring. To provide sufficient ion complexation ability, both compounds were decorated with four bulky tert-butyl groups fixing the required positions of the phenolic oxygen atoms in space. The other group of heteroatoms was required "to wrap" the metal ion more tightly. This set consisted either of the two amide nitrogen atoms and two carboxylic 
acid oxygen atoms in the case of compound $\mathbf{2 4 b}$, or only of the amide nitrogen atoms (for compound 24c). For compounds 24b-c, the principle of sensing was different from that of 24a, although the calcium coordination also caused a conformational change of the pendant group (in this case, the pyrene fragments). This kind of spatial rearrangement produced other photophysical changes, such as a decrease in the intensity of the intramolecular excimer emission band at $480 \mathrm{~nm}$ and, at the same time, an increase in the intensity of the monomer emission band at $397 \mathrm{~nm}$. The changes of the fluorescence intensity ratios of the monomerexcimer emission $\left(I_{\mathrm{M}} / I_{\mathrm{E}}\right)$ of PyCalix varied from 2.4 to 12.2 (in the range of calcium concentration $10^{-7}-10^{-5} \mathrm{M}$ ); the stability constant of the calcium complex with sensor $\mathbf{2 4 c}$ being $1.4 \times 10^{6}$. As concerns compound $\mathbf{2 4 b}$, a PET process may also partially explain its sensitivity mechanism, because of the presence an additional methylene group between the pyrene ring and the amido group in compound $\mathbf{2 4 b .}$

\subsection{Podands as a coordination moiety for calcium sensors}

Upon coordination with calcium cations, open-chain polyethers change their molecular geometry and their photophysical properties. These changes can be employed in the evaluation of such ion concentrations.

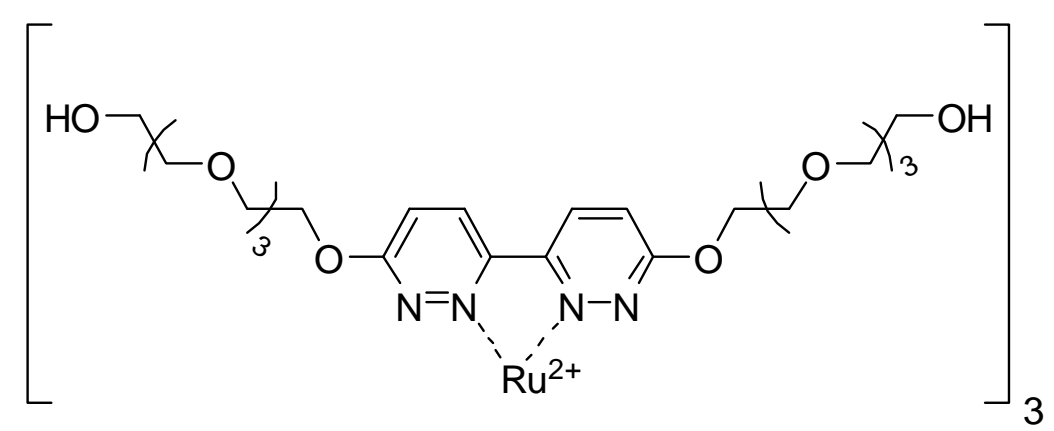

25

Figure 29. A ruthenium complex which is sensitive to the presence of calcium. ${ }^{[83]}$

Compound 25 was one of the first podand calcium indicators. It represents a highly watersoluble ruthenium complex. ${ }^{[83]}$ Initially, it was created not as a sensor and, therefore, the sensitivity for calcium was poorly investigated. The complex 25 showed a satisfactory increase in fluorescence intensity (1.3 times) upon calcium saturation (at $0.65 \mathrm{mM}$ ). The selectivity of compound $\mathbf{2 5}$ for calcium was very high (in comparison with other cations). For example, the stability constant of the calcium complex was 100 times higher than the stability constant of the complex with magnesium. 


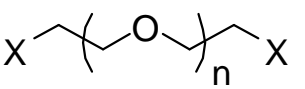

26a: $\mathrm{n}=3, \mathrm{X}=\mathrm{R}^{1}$;

26b: $2 N P 5 N: n=4, X=R^{2}$;

26c: $2 N P 6 N: n=5, X=R^{2}$.<smiles></smiles>

Figure 30. Calcium sensors with open-chain polyether moieties as chelators.

Another sensor 26a (Figure 30), was reported by Kakizawa et al. ${ }^{[84]}$ Upon calcium complexation, compounds $\mathbf{2 4 c}$ and 26a have the same fundamental states (monomer and intramolecular dimer). In the presence of calcium, the latter compound transforms itself from a monomer (emission maximum $400 \mathrm{~nm}$ ) to an intramolecular dimer (em. max. $490 \mathrm{~nm}$ ), i. e. in the opposite direction than compound $\mathbf{2 4 c}$ does. It is possible, because the main conformation of the molecule 26a in solution is stretched and, upon complex formation this molecule "coils up", so that the anthracene residues oppose each other. In this state, compound 26a can very easily form an inner excimer upon light excitation. Along with the red shift, an 8-fold decrease in fluorescence intensity of the short wavelength band and a simultaneous 8-fold enhancement of the long wavelength band were observed. However, the selectivity of this indicator is not very good: the value of the stability constant for the calcium complex is only 3.5 and only 5 times higher than those for strontium and magnesium, respectively.

The performance of compounds 26b-c (see Figure 30) is based on the same principles, as that for compound 26a. The influence of the polyether chain length and the nature of chromophores on the fluorescence response upon metal-ion coordination were reported. ${ }^{[85]}$ It was found that only calcium and barium increase the intensity of the maxima of the excimer bands (at $400 \mathrm{~nm}$ for $\mathbf{2 6 \mathbf { b }}$ and $415 \mathrm{~nm}$ for $\mathbf{2 6 c}$ in the case of $\mathrm{Ca}^{2+}$ ) and, at the same time, decreased the intensities of the monomer bands (at $360 \mathrm{~nm}$ for both sensors) by the same order of magnitude (2-3 times). A higher fluorescence intensity was observed in the case of a 2-naphthalenecarboxy chromophore present (compound 26b-c) in comparison with the 1naphthalenecarboxy analogue (not shown). The reason is that the carboxy groups in the $\alpha-$ substituted naphthalene fragments are twisted, and the conjugation is perturbed. The stability constants were higher for the longer chain with the $\beta$-naphthalene chromophore $\left(\log K_{\mathrm{s}}(\mathbf{2 6 b})\right.$ $\left.=4.19 ; \log K_{\mathrm{s}}(\mathbf{2 6 c})=4.95\right)$. 


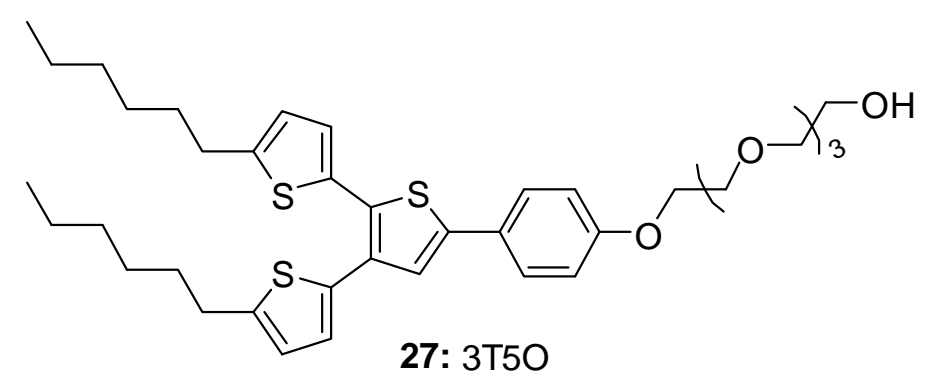

Figure 31. The podand 27. ${ }^{[86]}$

Another compound of this class is the terthiophene Dendron 27 with a tetraethylene glycol coordination residue, which was described in 2008. ${ }^{[86]}$ The indicator 27 has two absorption bands, one at 300 and one at $350 \mathrm{~nm}$, respectively. These bands do not change their positions in the presence of calcium. The spectrum of compound 27 has one fluorescence band at $460 \mathrm{~nm}$. This band undergoes a significant blue shift (to $320 \mathrm{~nm}$ ), and its intensity strongly increases upon calcium coordination (ca. 6 times). According to the interpretations of the authors, the addition of calcium salts produces a "twist" of the thiophene units within the chromophore moiety to form a complex with the cation. As a result, the large conjugated system shrinks to a smaller one, which is much more fluorescent and emits in the UV region. ${ }^{[86]}$ 


\section{Chapter 2. Synthesis and properties of new fluorescent calcium sensors}

\section{Search for an optimal coordinative part and its position within the phenylxanthene backbone}

The choice of the target structures for our research was based on their required properties and the detailed analysis of the available literature data. During the 35 years that passed from the first publication on the application of calcium indicators in biological research, more than a hundred new compounds have been created. All of these substances are sensitive to calcium. However, it is well known, that is impossible to use only one indicator for various applications. Therefore, it is often necessary to prepare new indicators for special purposes. Moreover, new research methods and objects in molecular biology require calcium indicators with novel properties. The required properties of the calcium sensors applicable in optical nanoscopy are:

1) excitation and emission maxima in the region above $450 \mathrm{~nm}$;

2) a "switching" possibility (e. g. due to the presence of a free carboxy group on the phenyl ring in the ortho-position to the xanthene fragment);

3) a high "brightness" of the dye (brightness is the product of the fluorescence quantum yield and extinction coefficient);

4) a certain sensitivity to calcium ion;

5) an ability to penetrate cell membranes;

6) a high photostability.

We will briefly recall the properties of the most "prominent" compounds from chapter 1 and discuss, if they correspond to the requirements mentioned above. Furthermore, we will show that the use of some known structural fragments in our own synthetic approach led to new target structures.

Each calcium indicator should be sufficiently water-soluble (as it has to "work" in an aqueous medium in the cell). The prominent sensors with BAPTA coordination moieties are soluble in water, because they contain carboxy groups in their coordination moieties. APTRA-indicators, with three carboxy groups in the coordination moiety, are also well enough water-soluble. However, the solubility in water of most of the crown ether-containing indicators is not very high. Two of them (16 and 22b) contain a phenol residue, which increases the solubility (but only very slightly). Among the calixarene sensors, compound 24b possesses two carboxy groups (and therefore, it is well soluble in water). One of the podand 
sensors $\mathbf{2 5}$ is hydrophilic. However, it is not possible to consider compound $\mathbf{2 5}$ as a leading structure for construction of novel sensors for biological research, because it is a complex of a heavy metal - ruthenium. As in the case of the BAPTA chelators, we have chosen carboxy groups as structural fragments, which not only increase hydrophilicity, but also provide additional coordinative properties.

Another important prerequisite is the necessity to apply visible light in experiments with cells, because UV light can damage organic compounds in the cells. Moreover, UV light produces an (auto) fluorescence background due to the emission from the nucleic acids and proteins. In the case of the BAPTA sensors, this problem was solved by careful choice of the fluorophore (optimized for compounds 5b-g, 6a-z, 6aa, 9a-h, 11c-e, 12, 13, 14a,b representing more than half of the BAPTA sensors). Xanthenes are the most popular fluorophores (e. g. rhodamines, fluoresceins, rhodoles) among the sensors mentioned above. Other classes of indicators (with an APTRA chelator) contain only ca. 1/3 of the substances which emit upon excitation with visible light: e. g. 15c (with the diarylpyrrolo[3,4c]pyrroldione core) and 15d-e (with the BODIPY ${ }^{\circledR}$ core). The main sensors with crown ether, with calixarene and with podand chelators are a priori not suitable for work in biological media, as only some indicators absorb in the visible region: e. g. 20a (stilbene with a benzoxazinone substituent), 20b (stilbene with a dicyanomethylene- $4 H$-pyran substituent).

In this work we used the most popular 9-phenylxanthene fluorophore which is easily synthesized and modified (Figure 32).

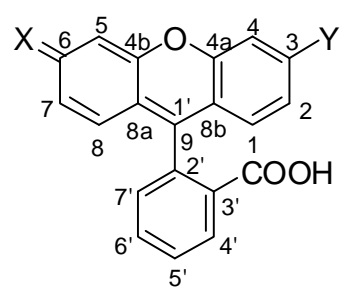

Figure 32. 9-(2-Carboxyphenyl)xanthene fluorophore of the present work $(X, Y=O ; X=O$, $\mathrm{Y}=\mathrm{N} ; \mathrm{X}, \mathrm{Y}=\mathrm{N})$.

The presence of a carboxy group in the ortho-position of the phenyl ring attached to the xanthene backbone is very important, as this group provides the switching possibility. ${ }^{[5]}$ Xanthene derivatives are found only in the BAPTA series of indicators. The potentially interesting compounds from which the photosensitive spiroamides ${ }^{[5]}$ or spirodiazaketones ${ }^{[6]}$ may be obtained are the BAPTA-indicators 6x, 9a-f, 9h, 11d-e. As a basic chromophore structure, all these indicators have a fluorescein (not rhodamine) residue. Though there are 
some methods for the preparation of spiroamides from fluorescein, ${ }^{[87]}$ but there is no guarantee, that these methods will be applicable for specific sensors.

Another important property is "the brightness" of a fluorescent sensor. In most cases, we refer to the values of the fluorescence quantum yields, because the extinction coefficients were found to be similar (ca. $1-3 \times 10^{4} \mathrm{M}^{-1} \mathrm{~cm}^{-1}$ ). Only a small part of the $\mathrm{Ca}$ sensors mentioned above have fluorescence quantum yields higher than $60 \%$. Such kind of sensors were referred to as green-emitting series $(\mathbf{9 a}-\mathbf{h}, \mathbf{1 1 d}-\mathbf{e})$ and crown ethers $(\mathbf{2 0 a}-\mathbf{b})$. Fluorescein and rhodamine are the chromophores of the "Green" series, the fluorophores of crown ethercontaining indicators are based on stilbenes with donor-acceptor pairs. In order to choose either fluorescein $(X=Y=O)$, or rhodol $(X=O, Y=N)$, or rhodamine $(X=Y=N)$ as a basic fluorophore (Figure 32), we have to consider another important prerequisite - the required fluorescent response upon calcium coordination. There are two kinds of this response: a) change of the fluorescence intensity; b) shift of the excitation and/or emission maxima. The best sensors (e. g. 4a or 5a) have a dual $(a+b)$ response. The type of the response depends on the working mechanism of the sensor (see section 2.1). There are PET sensors which increase or decrease their fluorescence intensity upon calcium coordination, and PCT sensors which are characterized by a shift of the maxima (sometimes accompanied by changes in the fluorescence intensity), as well as sensors with intramolecular formation / dissociation of excimers (drastic changes in the maxima positions). Among the BAPTA indicators, the PET sensors are Quin-2, 6a-z, 6aa-ab, 9a-h, 10a-c, 11a-e, 13 and the PCT sensors are 4a-e, 5a-o, 7a-k, 8a,b, 12, 14a,b. Among the APTRA sensors, the PET compounds are $\mathbf{1 5 a}, \mathbf{b}, \mathbf{d}, \mathbf{e}$, while $\mathbf{1 5 f}-\mathbf{j}$ are PCT compounds. Among the crown ethers, there are nearly equal amounts of PET (16, 17a-b, 19a-e, 23a-c) and PCT sensors (18a-f, 20a-b, 22a-b). Practically all calixarene indicators are based on the same sensing principle - the intramolecular excimer dissociation, and the podand indicators are based on an intramolecular excimer formation. It is interesting to note that the maximal increase in the fluorescence signal (in the case of PET sensors) was found for compound 11b (180 times). The greatest shift of the position of the fluorescent maxima (in the case of PCT sensors) was found to be $80 \mathrm{~nm}$ (for compound 18f). Compound 14a, with its highly fluorescent xanthene fluorophore, enables one to achieve a shift of both the excitation and emission maxima upon $\mathrm{Ca}$ complexation (whereas most indicators with xanthene fragments only increase their fluorescence intensity). Therefore, we can define the heteroatoms $\mathrm{X}$ and $\mathrm{Y}$ for our target structures in Figure 32, as $\mathrm{O}$ and N, on one hand, and N,N, on the other hand (Figure 33). 


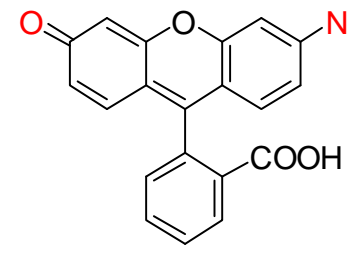

I

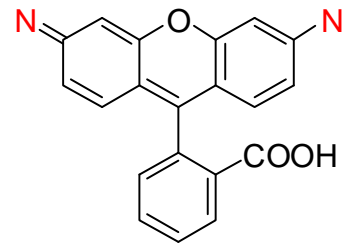

II

Figure 33. Rhodol (I) and rhodamine (II) as lead structures for creating new Ca sensors.

Most of the BAPTA indicators always possess good selectivity for calcium. The APTRA chelator, with its lower coordination number and only one nitrogen atom instead of two Natoms in BAPTA, is characterized by a lower selectivity to calcium (i. e., compound $\mathbf{1 5 b}$ has the same $K_{\mathrm{D}}$ values for $\mathrm{Ca}$ - and $\mathrm{Mg}$-ions). In fact, all indicators, with only crown ether residues as a coordination part, are not selective for calcium. However, for the vast majority of biological applications, it is enough to have an indicator with high selectivity for calcium in the presence of other biologically relevant cations $\left(\mathrm{Na}^{+}, \mathrm{K}^{+}, \mathrm{Mg}^{2+}\right)$. Thus, the three known compounds $18 \mathrm{~b}$ and 20a-b are suitable as lead structures for our requirements, though they are also sensitive to barium cations (which are absent in biological samples). In the series of calixarene sensors, it is known that compound $\mathbf{2 4 b}$ is equally selective for calcium and strontium. One of the podand sensors (26a) is known not to be selective for calcium, and another two (26b-c) have similar stability constants for complexes with calcium and barium (which is acceptable).

To define the coordination moiety more accurately, we have to consider one more requirement. The dissociation constant of the calcium-indicator complex has to be higher than $90 \mu \mathrm{M}$. This prerequisite is especially important for an investigation of calcium spikes by the STED microscopy. Indicators with lower $K_{\mathrm{D}}$ values would have been saturated long before the calcium level reaches its maximum value. Thus, indicators with lower $K_{\mathrm{D}}$ values could give inadequate results. From the whole family of BAPTA indicators, only compound 7e has a satisfactory $K_{\mathrm{D}}$ value of $550 \mu \mathrm{M}$. Among the APTRA sensors, there are already two suitable compounds 15d-e $\left(K_{\mathrm{D}} \approx 100 \mu \mathrm{M}\right)$. The crown ether indicators contain three compounds with $K_{\mathrm{D}}$ values of more than $90 \mu \mathrm{M}(\mathbf{1 8 b}-15 \mathrm{mM}, \mathbf{1 9 a}-630 \mu \mathrm{M}, \mathbf{2 0 b}-174 \mu \mathrm{M})$. All $\mathrm{Ca}$ indicators with calixarene and podand coordination moieties have dissociation constants lower than $90 \mu \mathrm{M}$. Therefore, the coordination site shown in Figure 34 was chosen as a starting point for our novel Ca sensors. 


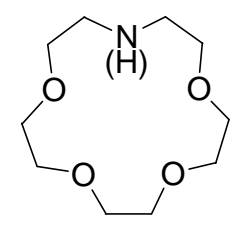

Figure 34. Coordination site for novel Ca sensors to be prepared.

The photostability of the fluorophore is important especially in the case of STED nanoscopy, where the excited state is depleted with a high-power laser. Xanthenes were the most photostable fluorophores used in the BAPTA indicators. The best APTRA indicators 15d-e were constructed with BODIPY $^{\circledR}$ fluorophores which are more photostable than fluorescein.

Penetration through the cell membrane is another very important factor which plays a key role in the design and optimization of the new calcium sensors. As it was mentioned in section 2.4 of the Chapter 1, the BAPTA indicators (and probably also the APTRA sensors) pass through cell membranes, if their negatively charged $\mathrm{COO}^{-}$groups are converted to neutral acetoxymethyl esters, the phenolic $\mathrm{OH}$ groups are acetylated, and the sterically hindered carboxy group in the $o$-position to the xanthene ring forms the internal 5 -membered lactone. This extensive acetoxymethylation removes all negative charges, the presence of which slows down the transport through a cell membrane. Deesterification is achieved inside a cell by action of enzymes (e. g. esterases). For other indicators this problem may be solved similarly (if they contain carboxy groups). To increase the permeability of one of our target compounds we used methyl groups (not ethyl groups) at nitrogen atoms (see Figure 35). It is known that tetramethylrhodamine derivates pass through cell membranes easier than the derivatives of rhodamine $\mathrm{B}$.

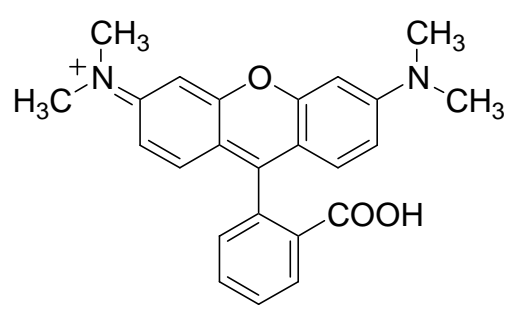

Figure 35. Tetramethylrhodamine as a building block for the sensor II.

To form the final structures, it is necessary to connect or fuse the fluorophore (xanthene) and the ionophore (azacrown). To distinguish between connection and fusion, we consider the compounds 29 (in which the crown ring connected with the benzene ring via one heteroatom) and $\mathbf{2 8}$ (in which two heteroatoms of the crown ether are connected with the benzene ring). 


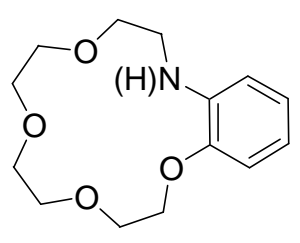

28

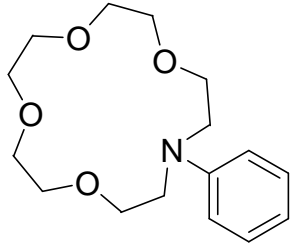

29

Figure 36. Azacrown ethers 28, 29.

As it was mentioned in the section 2.6 of Chapter 1, upon coordination to the crown ether ring, the electron-withdrawing properties of the calcium cation, cause various spectroscopic changes (e. g. shift of the positions of the absorption and fluorescence maxima or increase/decrease of their intensity). Due to the calcium coordination, the electron density on the aromatic system connected with heteroatoms is reduced. Obviously, if we bind the calcium cation with two heteroatoms (compound 28), the changes of the electron density on the aromatic system will be stronger, and therefore, the spectroscopic response has to be stronger.

There are several possible positions at which the xanthene core may be fused with the crown-ether system. However, not all of them are readily available. For example, fusion through the positions $1,2(7,8)$ and $6^{\prime}, 7^{\prime}$ are unfavorable, because the positions $1(7)$ and $7^{\prime}$ are sterically hindered (see Figure 32), and it is very difficult to integrate a crown ether using one of these positions. In the case of the target sensor I, positions 2(7), 3, 4(5), and 4', 5', 6' are available for the fusion with the crown ether macrocycle. We fused the crown-ether residue with the xanthene core through the positions 6 and 7 of the latter. As a model compound, we used the sensor 14a in order to provide similar spectroscopic changes (the shift of the fluorescence spectrum maximum) upon complexation with calcium.

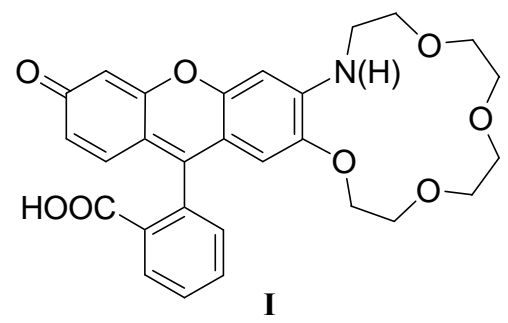

Figure 37. Incorporation of the crown-ether moiety into the sensor $\mathbf{I}$.

Another argument in favor of the structure I (as a model compound, see Figure 37) is the simplicity of the synthetic procedures compared to those associated with other substitution patterns of the xanthene backbone. 
The second type of a target sensor compound II, is presented in Figure 38. The crownether residue is fused with the positions $4^{\prime}$ and $5^{\prime}$ of the phenyl ring attached to the xanthene fragment. The main reason for chosing this kind of substitution pattern is the availability of compounds with 1,2,4,5-tetrasubstituted phenyl rings in comparison with 1,2,3,4-substituted benzene rings.

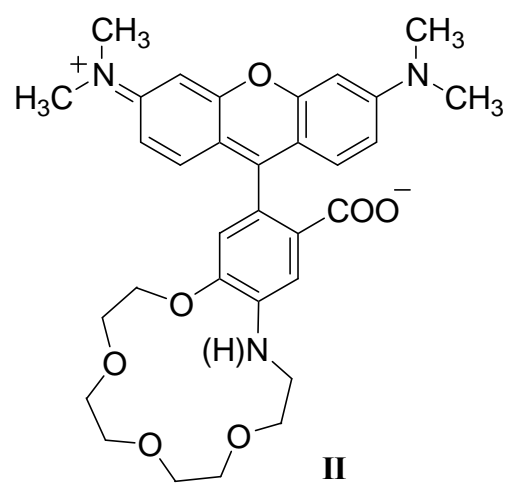

Figure 38. Incorporation of the crown ether residue into sensor II.

An important feature is the nature of the substituents at the nitrogen atom of the azacrown ether ring. It is well-known, that the presence of NH-groups (but not NR-groups) makes the azacrown ether (Figure 38) less susceptible for coordination with calcium cations. ${ }^{[88]}$ Therefore, we prepared indicators with an ethyl group (compounds Ib and IIa) and sensors with a carboxymethyl residue (compounds Ic and IIb). The carboxymethyl group has two important properties: a) it represents an additional coordinative site which may increase the selectivity to calcium; b) it substantially increases the solubility in water. An additional carboxy group in the phenyl ring attached to the xanthene fragment also increases the hydrophilicity. We prepared the model compound Ia for comparison, though this substance is deprived of an additional substituent at the nitrogen atom.

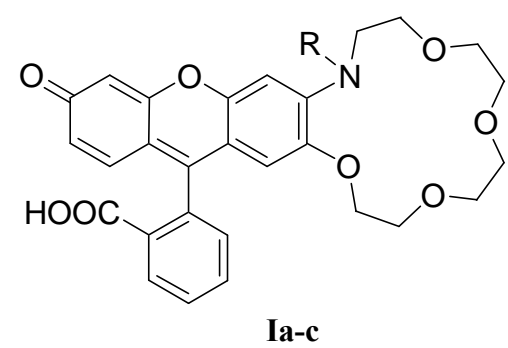

Ia: $\mathrm{R}=\mathrm{H}$

Ib: $\mathrm{R}=\mathrm{Et}$

Ic: $\mathrm{R}=\mathrm{CH}_{2} \mathrm{COOH}$

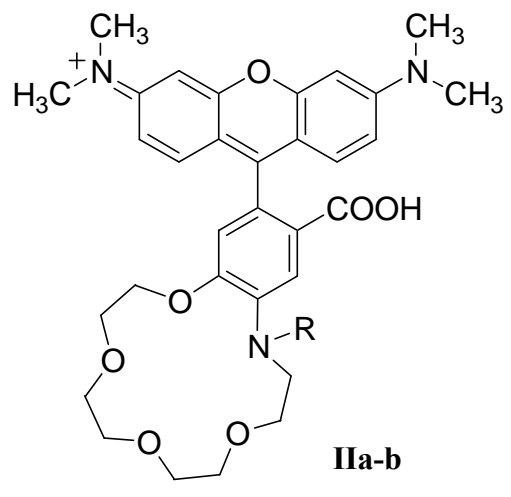

IIa: $\mathrm{R}=\mathrm{Et}$

IIb: $\mathrm{R}=\mathrm{CH}_{2} \mathrm{COOH}$

Figure 39. Target sensors Ia-c, IIa-b. 


\section{Model compounds}

Before we embarked on the synthesis of target compounds Ia-c, we had prepared some model compounds: a) a rhodamine with an alkoxy substituent in the ortho-position to the amino group; b) spiroamides with a "closed" rhodol fragment (cf.:[5]).

A conventional synthetic route to rhodols with a carboxy group starts with an acylation of resorcine or $m$-aminophenol with phthalic anhydride (30) followed by the reaction with a second molecule of $m$-aminophenol or resorcine (see Scheme 7).

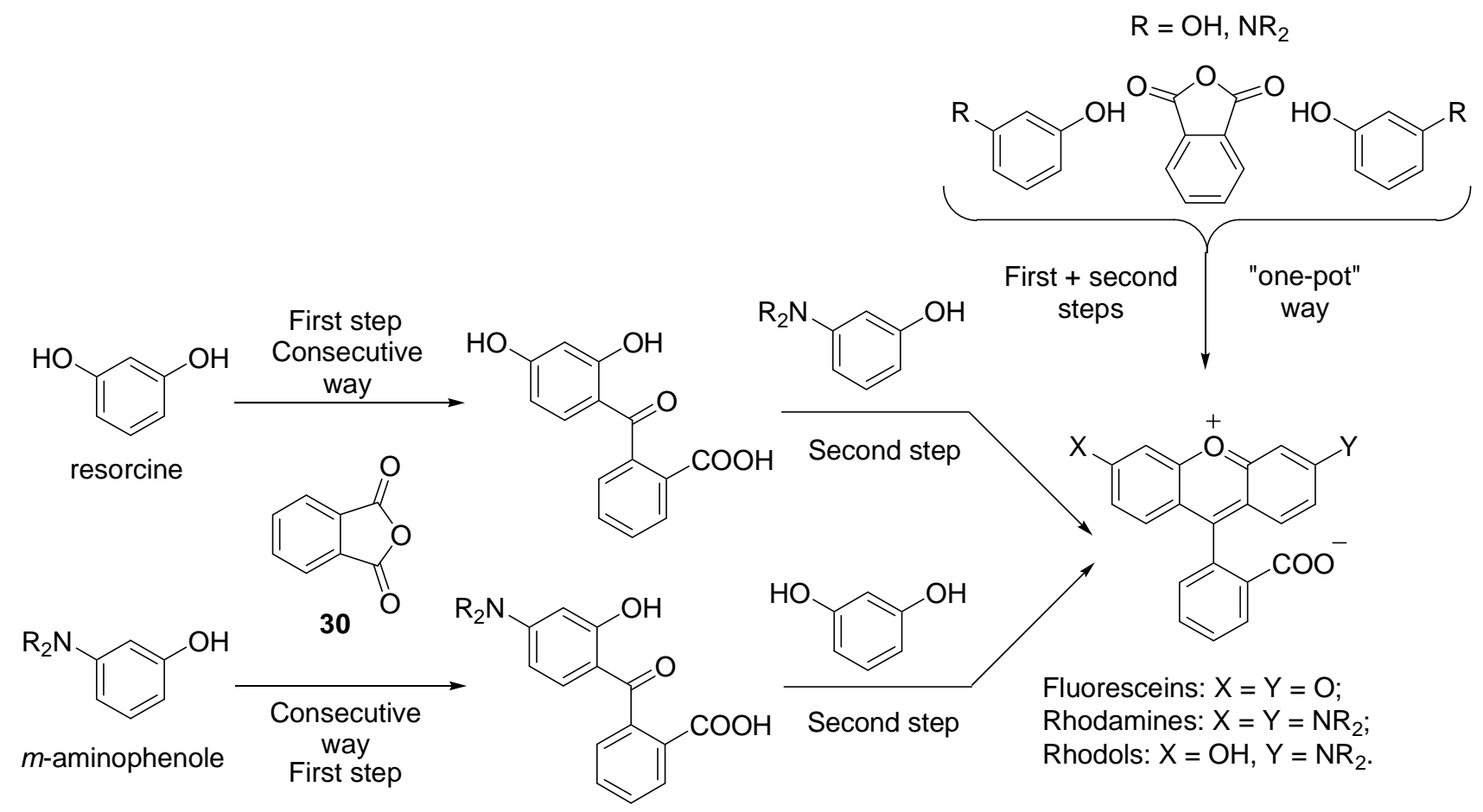

Scheme 7. Various synthetic routes towards xanthene dyes.

3-(N,N-Diethylamino)-4-methoxyphenol (compound 31 in Figure 40) was prepared as a model compound from the commercially available acetanilide $\mathbf{3 2}$ which was deacetylated by heating in aqueous $\mathrm{HCl}$ under reflux.<smiles>CCNc1cc(O)ccc1OC</smiles>

31

Figure 40. Model compound 31.

The aniline $\mathbf{3 3}$ was obtained in good yield and then diazotized. The aryldiazonium hydrogen-sulfate 34 could be converted into the phenol 31 only by prolonged boiling in 
aqueous solution. ${ }^{[89]}$ Other methods (adding of the diazonium solution into the boiling $67 \%$ sulfuric acid, ${ }^{[90]}$ or decomposition in the presence of $\mathrm{Cu}\left(\mathrm{NO}_{3}\right)_{2}$ and $\mathrm{Cu}_{2} \mathrm{O}^{[91]}$ ) failed to provide the required phenol 31.

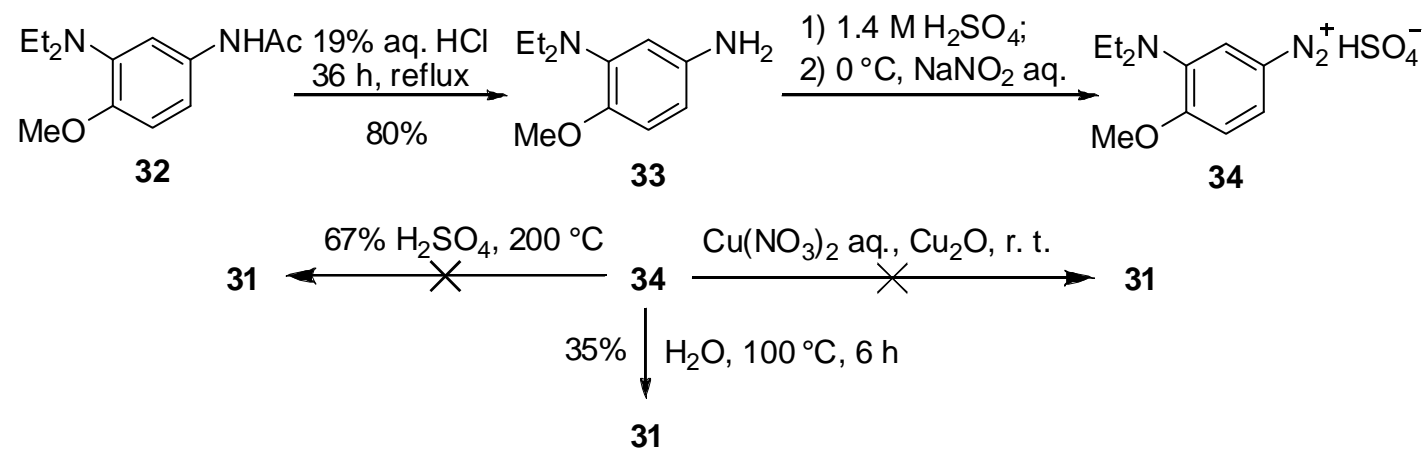

Scheme 8. A successful route to the phenol 31 .

The phenol 31 was used for the preparation of rhodamine 35 (Scheme 9). Unfortunately, the yield of the dimethoxyrhodamine 35 was only moderate $(18 \%)$. As a rule, conventional condensation methods require harsh reaction conditions for the second step (Scheme 7): high temperatures (up to ca. $200^{\circ} \mathrm{C}$ ) and strong acids (e. g. phosphoric acid, 50\% aq. sulfuric acid). For the model reaction (compound $\mathbf{3 0}+$ compound $\mathbf{3 1}$ ) the mildest conditions were used: heating overnight in refluxing propionic acid with $p-\mathrm{Tos} O H \cdot \mathrm{H}_{2} \mathrm{O}$ as a catalyst. ${ }^{[2]}$

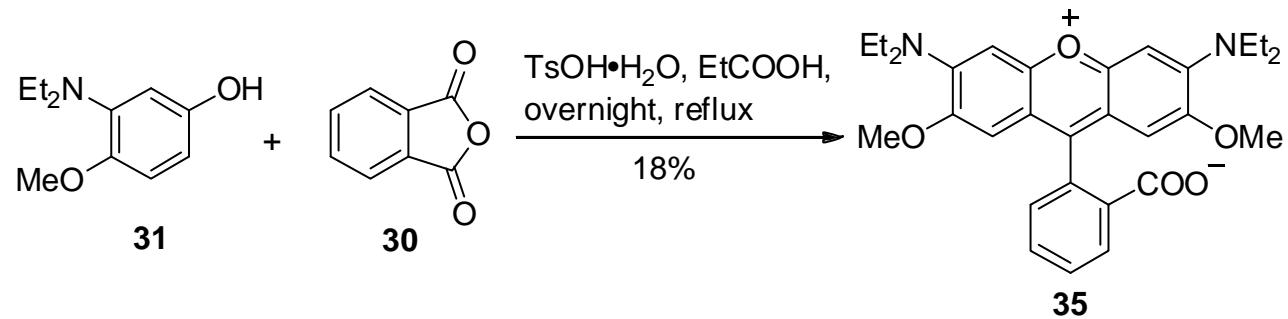

Scheme 9. Synthesis of rhodamine 35 .

In order to check the possibility of synthesizing spiroamides from rhodols compounds 39 ${ }^{[93]}$ were prepared according to the known method. ${ }^{[93]}$ Towards that end, 3-N,Ndiethylaminophenol 36 was acylated with phthalic anhydride 30 in a non-catalytic FriedelCrafts reaction to produce the benzophenone derivate $\mathbf{3 7}$ in good yield. The next step was the reaction of resorcinol $38-\mathrm{H}$ with the benzophenone 37 , and this was succeeded by an intramolecular condensation of the intermediate product at high temperature under acid catalysis, yielding $83 \%$ of rhodole $39-\mathrm{H}$. 


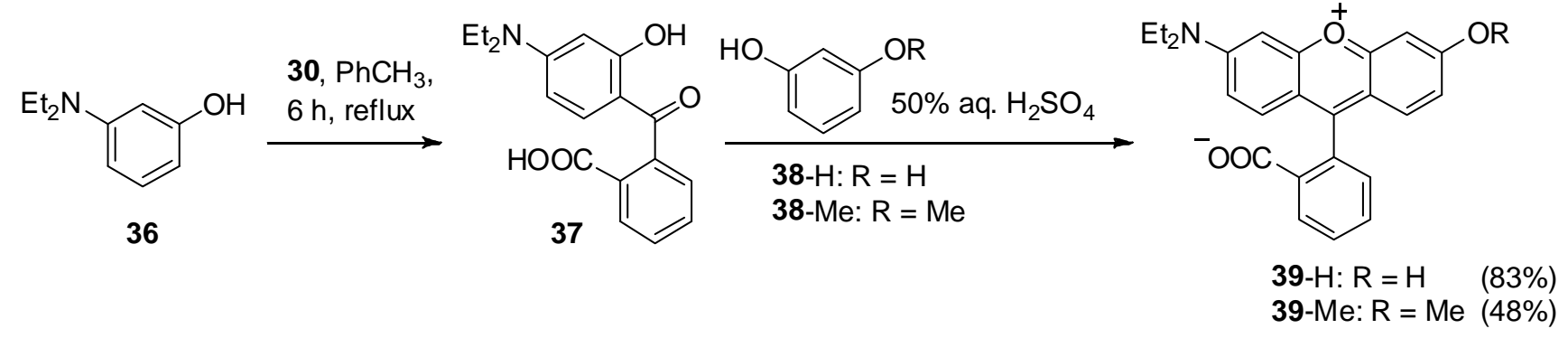

Scheme 10. Synthesis of the model rhodoles 39-H and 39-Me.

For preparation of the other model compound - the rhodole spiroamide $40-\mathrm{H}$ - the carboxy group in compound 39-H would have to be converted it into the corresponding acid chloride, ${ }^{[5]}$ which is impossible due to the presence of the free hydroxy group that may react with an acid chloride or may even be converted into the corresponding aromatic chloride (see Figure 41).<smiles>CCN(CC)c1ccc2c(c1)Oc1cc(Cl)ccc1C21OC(=O)c2ccccc21</smiles>

Figure 41. Possible product upon treatment of compound 39-H with phosphorus oxychloride $\left(\mathrm{POCl}_{3}\right)$.

To circumvent this problem, compound 39-Me was prepared (Scheme 10) and successfully converted into the acid chloride 41-Me (Scheme 11). The latter reacted with aniline and, in the presence of $\mathrm{Et}_{3} \mathrm{~N}$, gave the spiroamide 40-Me. Boron trichloride was used to remove the methoxy group ${ }^{[94]}$ to yield the required spiroamide 40-H (Scheme 11). 
<smiles>CCN(CC)c1ccc2c(-c3ccccc3C(=O)[O-])c3ccc(OC)cc3[o+]c2c1</smiles>

39-Me<smiles>CCN(CC)c1ccc2c(c1)Oc1cc(O)ccc1C21c2ccccc2C(=O)N1c1ccccc1</smiles>

$40-\mathrm{H}$

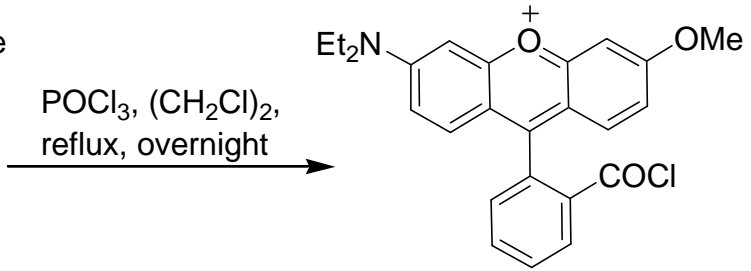

41-Me

$88 \% \mid \begin{aligned} & \mathrm{PhNH}_{2}, \mathrm{Et}_{3} \mathrm{~N}, \\ & \mathrm{MeCN}, 4 \mathrm{~h}, \text { reflux }\end{aligned}$<smiles>CCN(CC)c1ccc2c(c1)Oc1cc(OC)ccc1C21c2ccccc2C(=O)N1c1ccccc1</smiles>

40-Me

Scheme 11. Synthesis of the rhodole spiroamide $40-\mathrm{H}$.

\subsection{Spectroscopic properties of the model compounds}

For the model compound 35, interesting spectroscopic properties were observed. The fluorescence quantum yield of this compound was found to be only $4.5 \%$ in ethanol and $0.9 \%$ in aqueous solution. These values are much lower than those for the similar compound without methoxy groups - Rhodamine B (65\% in EtOH and $31 \%$ in water). ${ }^{[95]}$<smiles>CCN(CC)c1ccc2c(-c3ccccc3C(=O)[O-])c3ccc(N(CC)CC)cc3[o+]c2c1</smiles>

Rhodamine B<smiles>CCNc1cc2[o+]c3cc(N(CC)CC)c(OC)cc3c(-c3ccccc3C(=O)[O-])c2cc1OC</smiles>

35

Figure 42. The structures of Rhodamine B and compound 35.

A sharp decrease in the fluorescence quantum yield may be attributed to the higher rotational dissipation of energy of the molecule in the excited state. The methoxy group in the $o$-position of the diethylamino group could assist the rotation of the diethylamino group from being coplanar with the aromatic ring into an out-of-plane conformation. Therefore, the fraction of the non-radiative de-excitation processes becomes higher, and then the quantum yield decreases. 

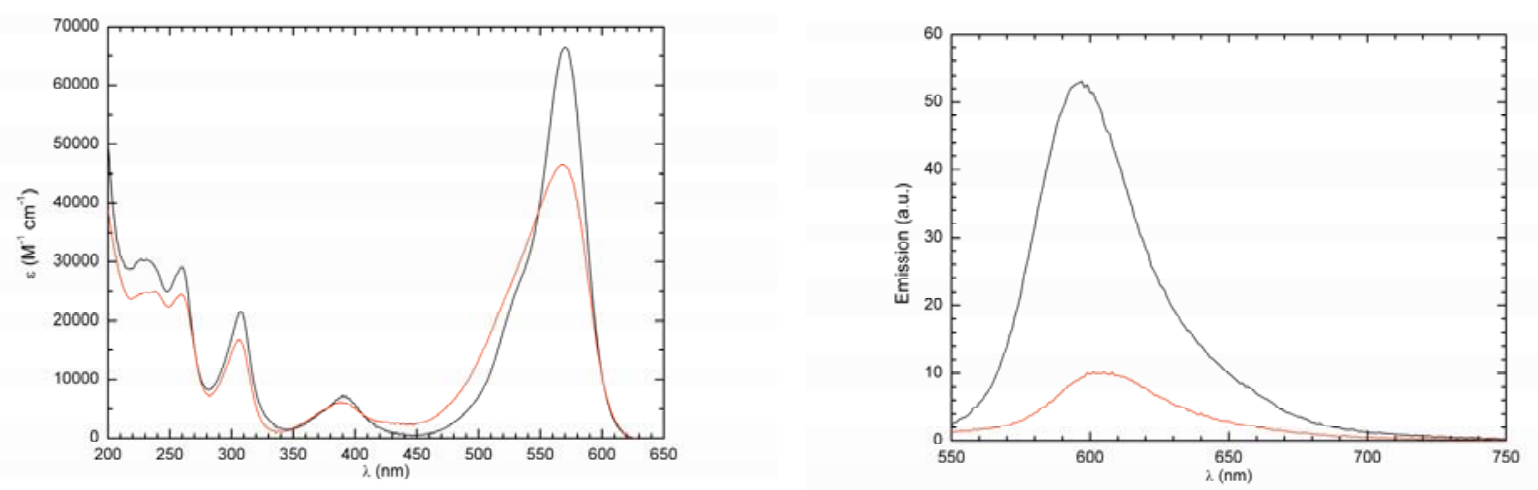

Figure 43. Absorption and fluorescence spectra of compound 35 in ethanol (black lines, $\lambda_{a b s}^{\max }=570 \mathrm{~nm}, \varepsilon=66500 \mathrm{M}^{-1} \mathrm{~cm}^{-1}, \lambda_{f l}^{\max }=596 \mathrm{~nm}$ ) and in water (red lines, $\lambda_{a b s}^{\max }=568 \mathrm{~nm}$, $\left.\varepsilon=47000 \mathrm{M}^{-1} \mathrm{~cm}^{-1}, \lambda_{f l}^{\max }=604 \mathrm{~nm}\right)$.

The absorption and emission spectra of compound 35 show bathochromic shifts in comparison with those of Rhodamine B (in ethanol: $\lambda_{a b s}^{\max }=542 \mathrm{~nm}, \lambda_{f l}^{\max }=568 \mathrm{~nm}$, in water: $\left.\lambda_{a b s}^{\max }=553 \mathrm{~nm}, \lambda_{f l}^{\max }=581 \mathrm{~nm}\right)$, which can be attributed to the presence of the donor methoxy group.

To check the photoswitching ability of the second series of model compounds $\mathbf{4 0}-\mathrm{H}$, the samples were illuminated with UV light, and then the emission spectra were recorded.

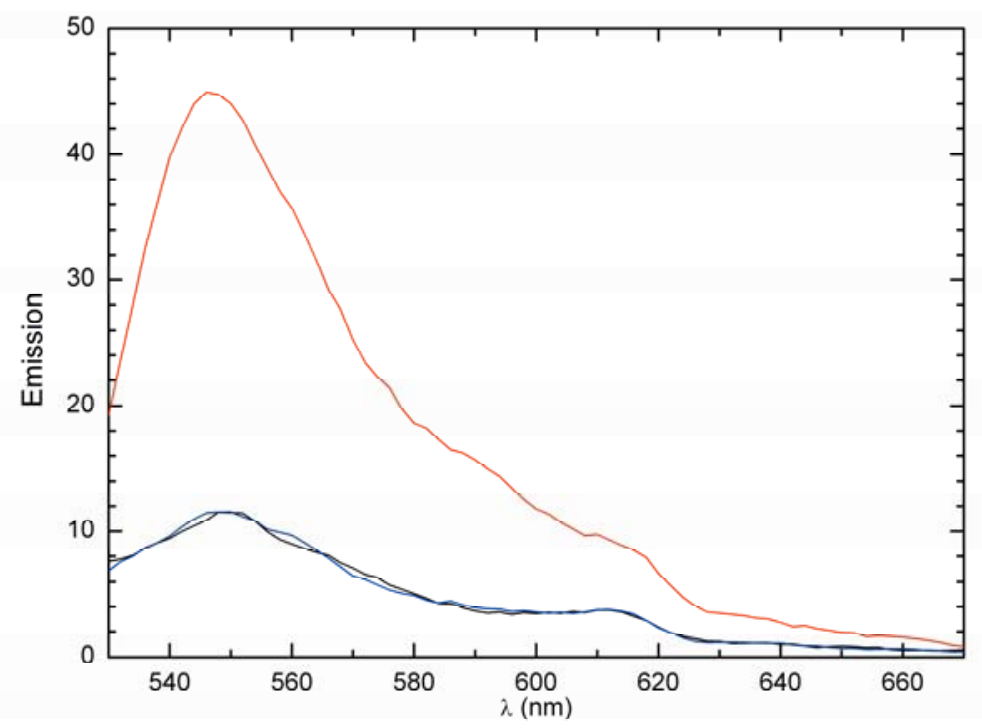

Figure 44. Emission spectra of compound 40-H in toluene (excitation wavelength: $520 \mathrm{~nm}$ ). Black line: sample stabilized in the dark; Red line: sample irradiated for one minute with UV 
light, selected with a band-pass filter centered at $366 \mathrm{~nm}$ (UG1 - $3 \mathrm{~mm}$, Schott); blue line: sample allowed to relax in the dark for one minute.

The emission maxima of the open form of the rhodole spiroamide $40-\mathrm{H}$ was observed at $546 \mathrm{~nm}$. The efficiency of the opening reaction of the rhodole spiroamides was very low, and the thermal reclosing was fast. Only in toluene, the closing reaction was slow enough, so that it was possible to accumulate the open form with prolonged irradiation; but the amount of the open form was still very low. Nevertheless, the switching possibility for this kind of compounds was confirmed.

\section{Synthesis of rhodole derivatives with a crown-ether moiety fused with the xanthene fragment}

A retrosynthetic analysis reveals that the skeleton of compounds Ia-c may easily be constructed from phthalic anhydride 30, resorcinol 38-H and the phenols 42a-c (see Scheme 12). These components should be combined sequentially (see Scheme 7).

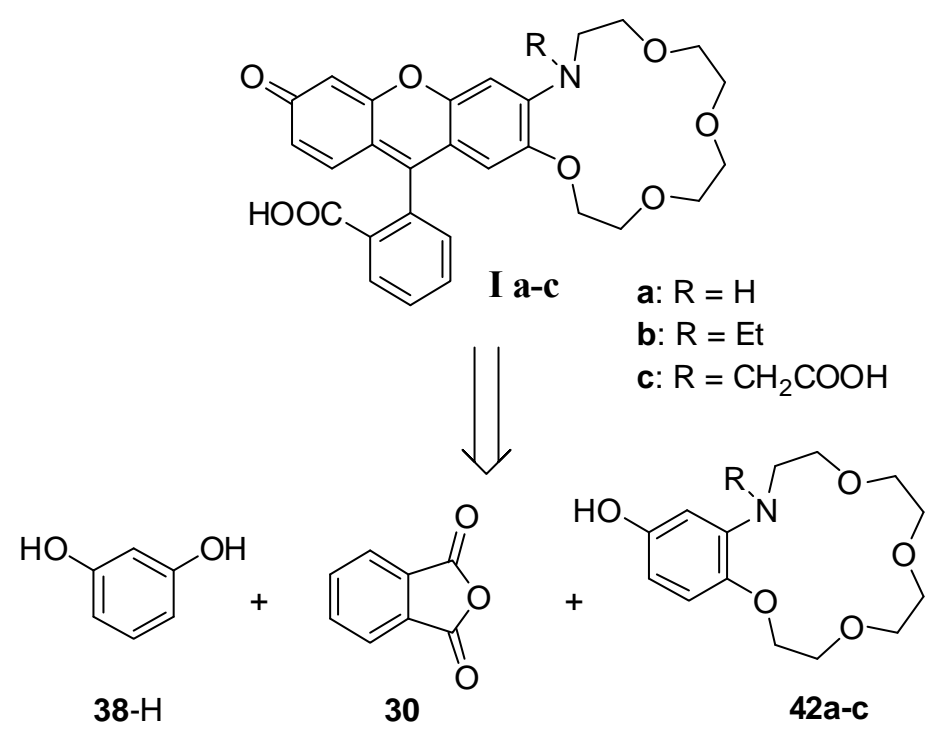

Scheme 12. A retrosynthetic consideration of the target structures Ia-c.

\subsection{Synthesis of the phenol building blocks via the unfunctionalized crown ether}

There are two principally different synthetic routes to compounds $\mathbf{4 2 a - c}$. The azacrown ring may be constructed first, and then the hydroxy group (or a precursor of it) may be 
introduced into the aromatic ring (Scheme 13). Another option is to use a precursor with a hydroxy group (or its equivalent), and then built up the crown-ether ring.

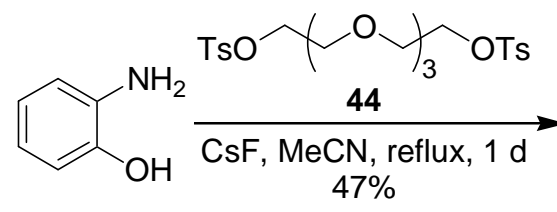

43

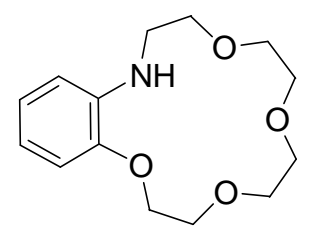

45

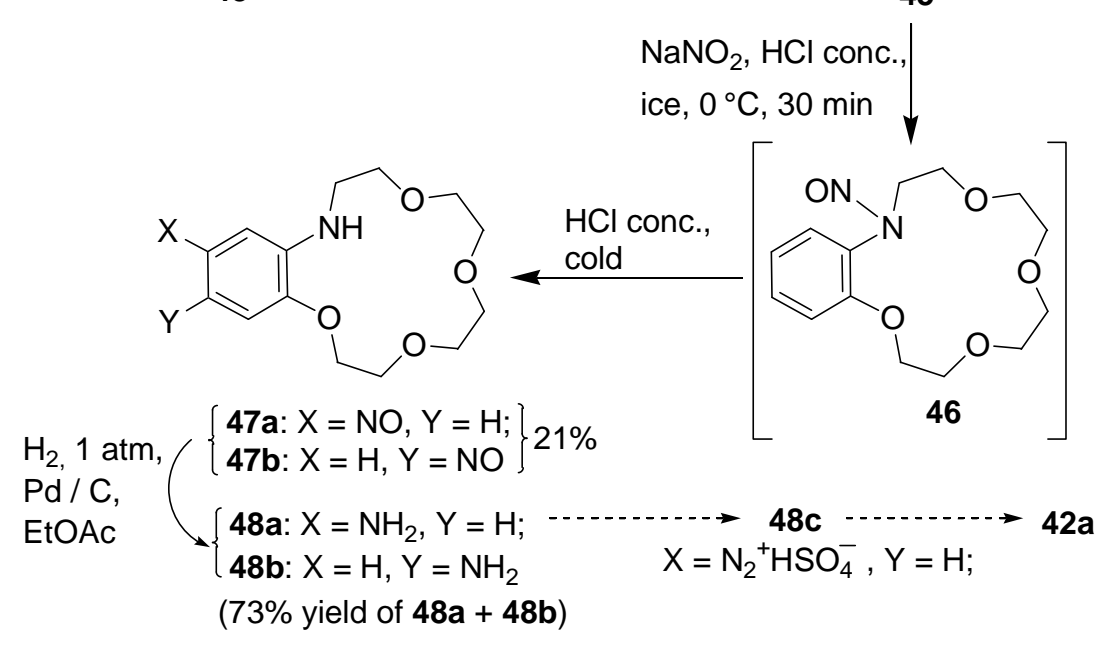

Scheme 13. Synthesis of the anilines $48 \mathrm{a}$ and $\mathbf{4 8 b}$.

The azacrown 45 was prepared from $o$-aminophenol (43) and tetraethyleneglycol ditosylate 44 according to a known procedure. ${ }^{[96]}$ For the introduction of the hydroxy group into the aromatic ring of compound $\mathbf{4 5}$, the latter was first converted into the $N$-nitrosoamine 46. ${ }^{[97]}$ However, under conditions which provided the full conversion of amine 45, an unseparable mixture of two $C$-nitroso compounds $(47 \mathbf{a}+\mathbf{4 7 b})$ was formed. Obviously, in the course of this transformation, a rearrangement of the $N$-nitrosoamine into the $C$-nitroso derivatives took place. This rearrangement was found not to be regioselective. ${ }^{[97]}$ Reduction to the corresponding amines did not help to separate the regioisomers $48 \mathrm{a}$ and $\mathbf{4 8 b}$ (the target compound 42a is formed only from compounds $48 \mathrm{a}$ via $48 \mathrm{c}$ ). Therefore, we did not pursue this route any further.

\subsection{Attempted synthesis of phenol building blocks via the crown ether prepared from 2-amino-4-nitrophenol}

As we failed selectively to introduce the functional group into the required position of azacrown 45, we decided to construct the macrocycle starting from $o$-aminophenol with a functional group in the required position. 


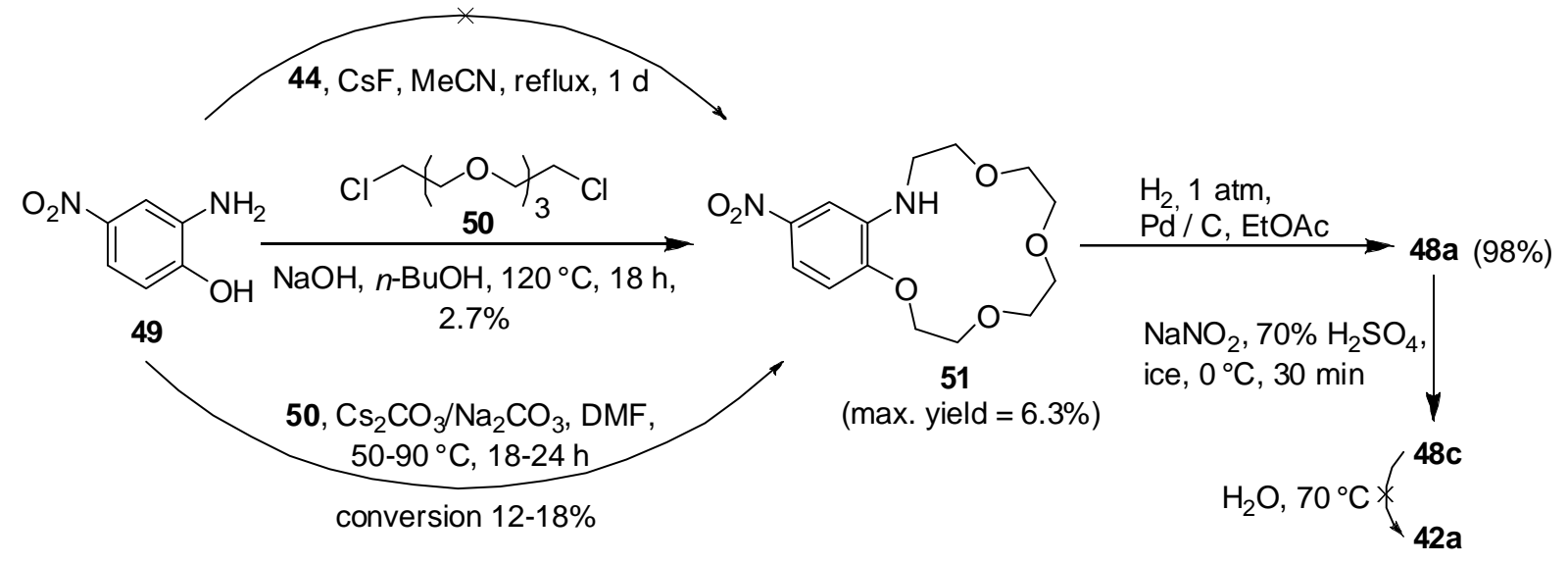

Scheme 14. An approach towards phenol 42a via the nitrobenzocrown 51.

Under the conditions of the successful preparation of the azacrown $45,{ }^{[96]}$ the desired macrocycle 51 could not be detected in the reaction mixture from compound $\mathbf{4 9}$ (Scheme 14). Under more drastic conditions $\left(\mathrm{NaOH}\right.$ in $n$-butanol at $\left.120{ }^{\circ} \mathrm{C}\right),{ }^{[97]}$ the yield of compound $\mathbf{5 1}$ was found to be very low $(2.7 \%$ after $18 \mathrm{~h})$. The maximal conversion of the initial aminophenol 49 was found to be $18 \%$ (with 2 equiv. of $\mathrm{Na}_{2} \mathrm{CO}_{3}$ as a base in DMF at $90{ }^{\circ} \mathrm{C}$ ). The preparative yield of the product (51) was only 6.3\%. Several hundreds of $\mathrm{mg}$ of compound 51 were prepared and reduced to the aniline 48a with hydrogen over palladium on charcoal. The aromatic amine 48a was converted to the diazonium hydrogen sulfate $48 \mathbf{c}$, which was heated in aqueous solution at $70{ }^{\circ} \mathrm{C}$. Unfortunately, the required phenol 42a was not detected in the reaction mixture. This route was not pursued any further, because the key intermediate $\mathbf{5 1}$ could not be prepared in an acceptable yield.

\subsection{Synthesis of the phenol building blocks by a ring-closing reaction of a tetraethylene glycol precursor}

Complications associated with the presence of the nitro group in the aromatic ring (prone to vicarious nucleophilic substitution), should be avoided and therefore, each synthetic step should involve only one active nucleophile and one active electrophile.

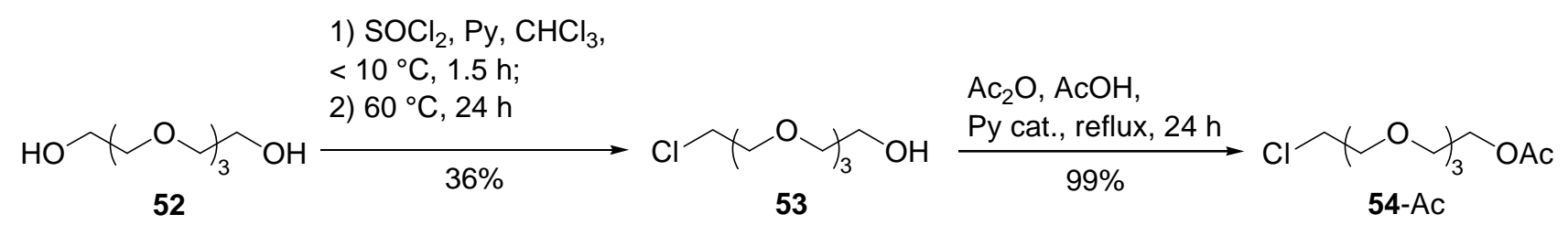

Scheme 15. Synthesis of the protected tetraethylene glycol building block 54-Ac. 
To generate a precursor for the crown-ether ring, tetraethylene glycol (52) was converted to the monochloride 53, ${ }^{[98]}$ and then to acetate 54-Ac (Scheme 15) with a good leaving group on one end and an appropriate protecting group on the other end.

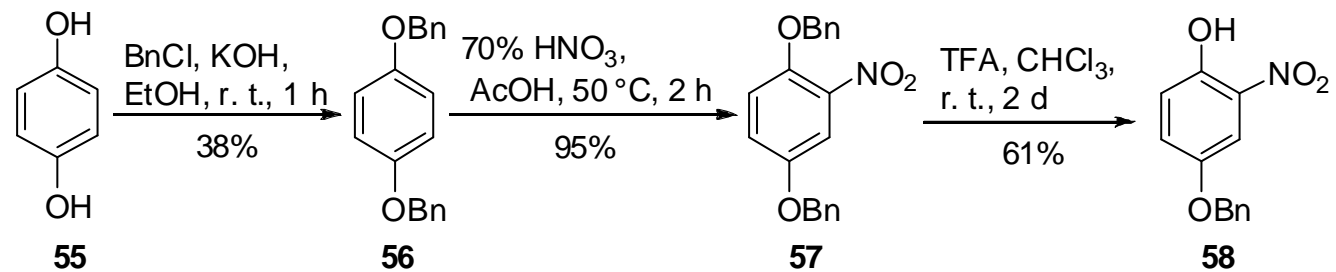

Scheme 16. Synthesis of the nitro phenol 58 as a second building block.

For the second building block, hydroquinone (55) was dibenzylated, mono-nitrated, and then the benzyl group at the oxygen atom in the $o$-position to the nitro group was selectively removed (Scheme 16). ${ }^{[35 b]}$

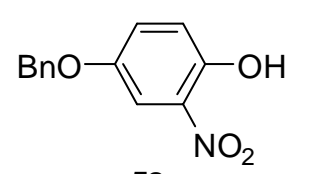

58 1) $t$-BuOK, DMF, $0{ }^{\circ} \mathrm{C}, 30 \mathrm{~min}$; 2) $10 \% \mathrm{KI}, 54-\mathrm{Ac}, 90^{\circ} \mathrm{C}, 3 \mathrm{~d}$ $82 \%$

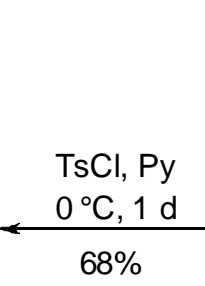
61<smiles>CC(=O)OCCOCCOc1ccc(OBr)cc1[N+](=O)[O-]</smiles>

59 $95 \%$ N $\mathrm{N}_{2} \mathrm{H}_{4}$, Ni-Raney, $\mathrm{MeOH}, 60^{\circ} \mathrm{C}, 2.5 \mathrm{~h}$<smiles>Nc1cc(OCc2ccccc2)ccc1OCCOCCO</smiles>

$60 \mathrm{a}$

Scheme 17. Synthesis of intermediate product ditosylate 61.

The building blocks 54-Ac and $\mathbf{5 8}$ were combined according to Scheme 17. Alkylation of the potassium salt of the nitrophenol $\mathbf{5 8}$ (produced in situ from equimolar amounts of potassium tert-butoxide and compound 58) with the chloride 54-Ac was carried out in the presence of potassium iodide as a catalyst. The nitro group of the obtained product $\mathbf{5 9}$ was reduced, ${ }^{[8]}$ and the acetoxy group was cleaved in a one-pot reaction with hydrazine in the presence of Raney-Ni. This transformation provided the amino alcohol 60a (95\%). The latter was treated with tosyl chloride in pyridine, and thus the ditosylate 61 was obtained in $68 \%$ yield. Attempts to increase the yield by using DMAP as a catalyst or by a step-wise tosylation, failed. 


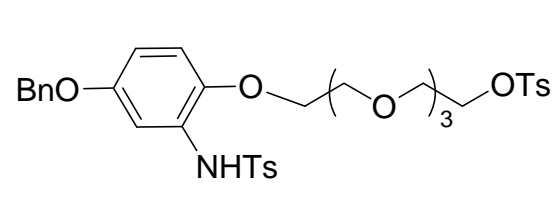

61

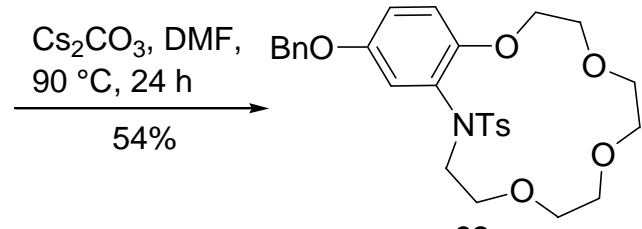

62
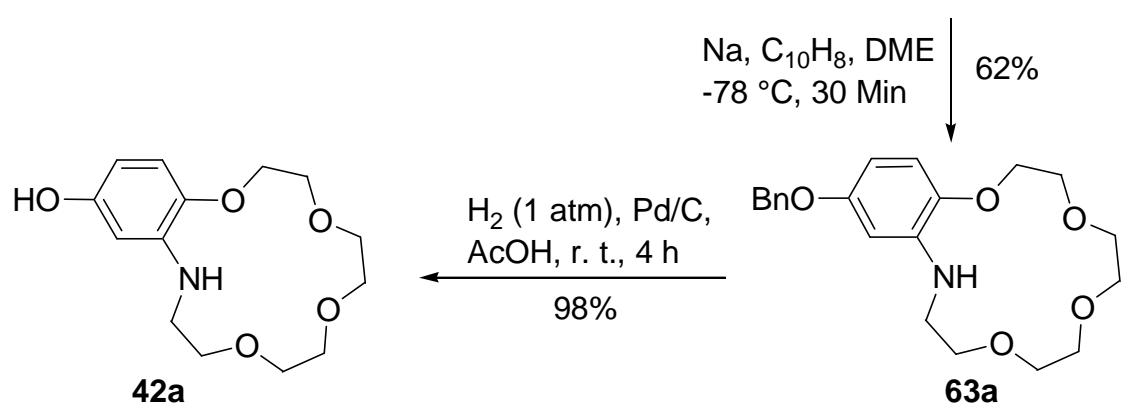

$63 a$

Scheme 18. The final steps in the preparation of the phenol 42a.

It is known from the literature, that under basic conditions tosylates may alkylate tosylamides to form $N$-tosyl derivatives of the secondary amines. ${ }^{[99]}$ These regularities allowed to perform a ring-closing reaction and obtain the $N$-tosylated crown ether $\mathbf{6 2}$. The detosylation was carried out under mild conditions ${ }^{[100]}$ (see Scheme 18), because the recommended conditions (conc. $\mathrm{H}_{2} \mathrm{SO}_{4}, 100{ }^{\circ} \mathrm{C}, 12 \mathrm{~h}$ ) ${ }^{[99]}$ are too harsh and would destroy the crown ether ring. Finally, compound 63a was debenzylated by the catalytic hydrogenation over palladium to give the target phenol 42a in nearly quantitative yield. Acetic acid was used as a solvent, ${ }^{[101]}$ in order to prevent deactivation of the catalyst by the liberated amine $\mathbf{4 2 a}$.

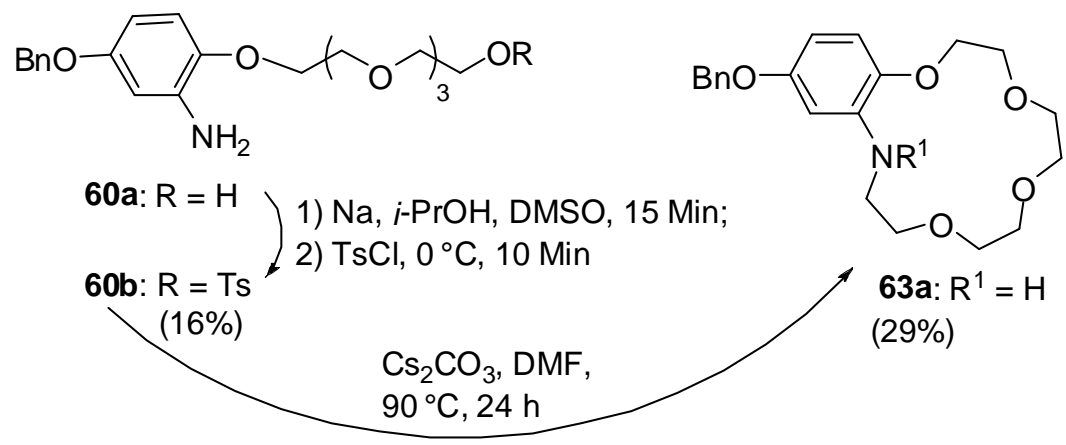

Scheme 19. Alternative way to the crown ether 63a.

An alternative route to the crown ether 63a (Scheme 19) would start with a cyclization of the monotosylate $60 \mathrm{~b}$. A potential advantage of this approach would be to avoid the tedious detosylation step. However, already the first step of this two-step sequence yielded only $16 \%$ of the monotosylate $\mathbf{6 0 b}$. The second step turned out to also be low-yielding (29\%), so that the total yield was only $5 \%$, which is much less than the total yield in the longer sequence $(23 \%)$. 


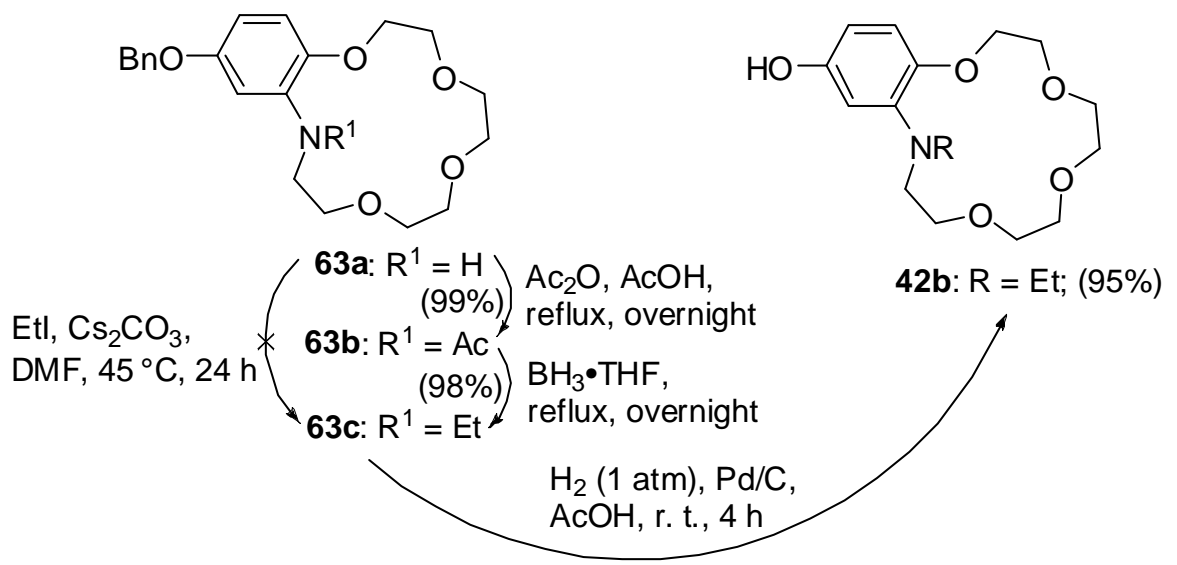

Scheme 20. Preparation of the $N$-ethyl derivative $\mathbf{4 2 b}$ of the crown ether with a free $\mathrm{OH}$ group.

For the synthesis of Ca sensors, we also needed the precursor 42b with an $N$-ethyl group and an unprotected phenolic hydroxy group. The direct alkylation of the starting compound 63a with ethyl iodide failed to produce the $N$-ethyl derivative 63c. Only the starting material was isolated. Therefore, we used an alternative acylation-reduction procedure ${ }^{[102]}$ which gave compound $63 \mathrm{c}$ in nearly quantitative yield. The final step - reductive debenzylation of the ether 63c into phenol 42b - was carried out successfully, and the target product was isolated in high yield (92\%).

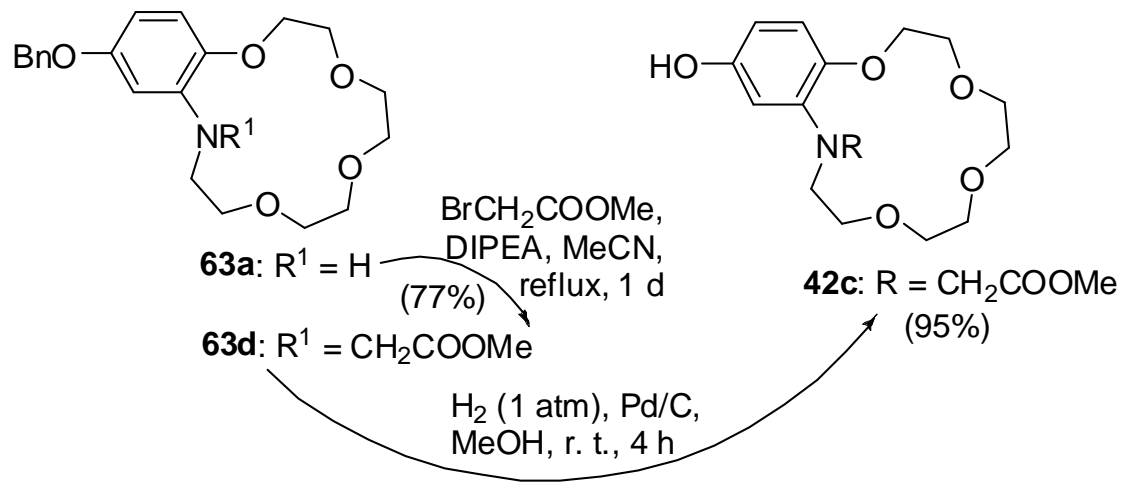

Scheme 21. Synthesis of the crown-ether building block with an additional coordination site (compound 42c).

The last starting material in this crown ether series - the phenol $42 \mathrm{c}$ - was prepared by alkylation of compound 63a with methyl-2-bromoacetate in acetonitrile. ${ }^{[64]}$ This procedure afforded the ester $\mathbf{6 3 d}$ which was debenzylated in $\mathrm{MeOH}$ to produce the required compound 42c in very high yield (95\%).

After the successful syntheses of the building blocks with azacrown-ether residues fused with the aromatic ring bearing the hydroxy group, the synthesis of phenol $\mathbf{6 8}$, in which an 
azacrown-ether residue is attached to the aromatic ring, was developed. This compound was necessary for the synthesis of the fluorescent dye $\mathbf{7 0}$ (see Scheme 24), in which the Ca chelator is directly connected to the $\pi$-system of the fluorophore.

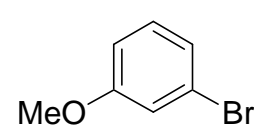

64

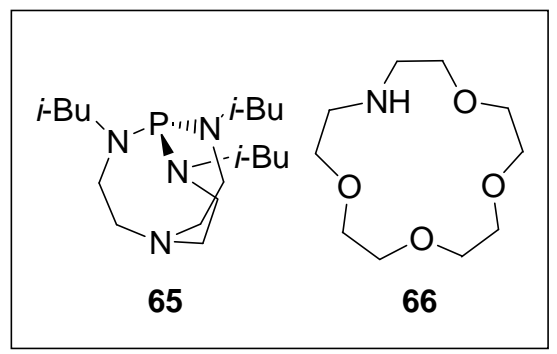
1.4 eq. $t$-BuONa,
1.2 eq. 66, 4 mol. \% 65 $1 \mathrm{~mol} \% \mathrm{Pd}_{2}(\mathrm{dba})_{3}$ toluene, $100{ }^{\circ} \mathrm{C}, 24 \mathrm{~h}$

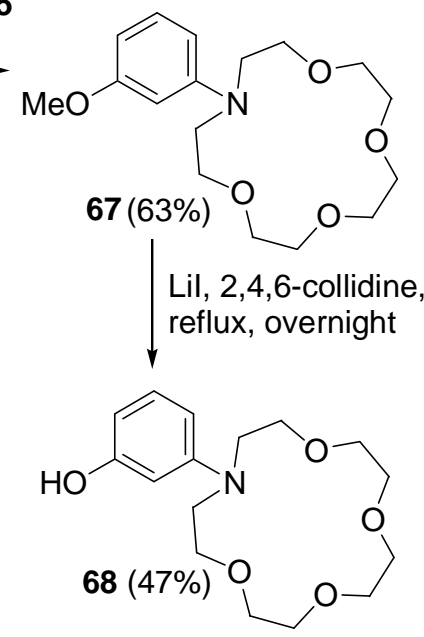

Scheme 22. Preparation of the building block 68 with an azacrown-ether residue attached to the aromatic ring.

The precursor of phenol 68 - compound 67 - was prepared by a Buchwald-Hartwig amination of $m$-bromanisol (64) with aza-15-crown-5 (66) as described. ${ }^{[103]}$ The yield of compound 67 was found to be high (63\%). The methoxy group in compound 67 could be cleaved selectively with lithium iodide in 2,4,6-collidine, ${ }^{[104]}$ and the required product $\mathbf{6 8}$ was isolated in moderate yield (47\%).<smiles>O=C1OC(=O)c2ccccc21</smiles>

30<smiles>Oc1cccc(O)c1</smiles>

38- $\mathrm{H}$<smiles>C[C@H]([Mg][Mg])[N+](=O)[O-]</smiles>

69

Scheme 23. Synthesis of the benzophenone 69.

"The second half" of the rhodoles Ia-c - the benzophenone derivative $\mathbf{6 9}$ - was prepared by Friedel-Crafts acylation of resorcinol $(\mathbf{3 8}-\mathrm{H})$ with phthalic anhydride $\mathbf{3 0}$ in the presence of aluminum chloride as a catalyst. ${ }^{[64]}$ The main reason, why we chose the combination of compound 69 with the crown ethers 42a-c (but did not prepare the corresponding benzophenones from phthalic anhydride and phenols 42a-c) is that we not only reduced the total number of synthetic steps, but also saved the valuable azacrown ethers $42 \mathbf{a}-\mathbf{c}$ and $\mathbf{6 8}$. 

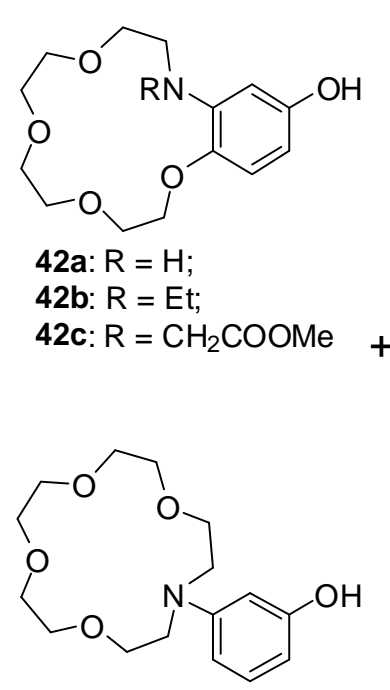

68<smiles>O=C(O)c1ccccc1C(=O)O</smiles>

69
$\mathrm{TsOH} \bullet \mathrm{H}_{2} \mathrm{O}, \mathrm{EtCOOH}$ overnight, reflux

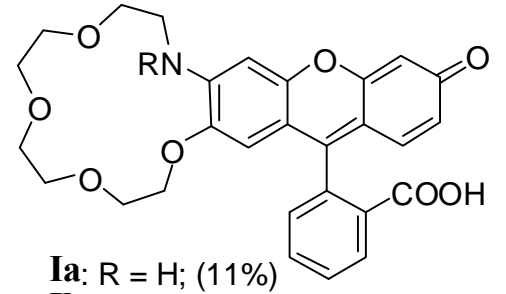

Ib. $\mathrm{R}=\mathrm{Et} ;(5.6 \%)$

Ic-Me-2/3TsOH: $\mathrm{R}=\mathrm{CH}_{2}$ COOMe, (18\%)

$\mathrm{K}_{2} \mathrm{CO}_{3}, \mathrm{MeOH}, 1 \mathrm{~h}$

- Ic: $\mathrm{R}=\mathrm{CH}_{2} \mathrm{COOH},(9.8 \%)$

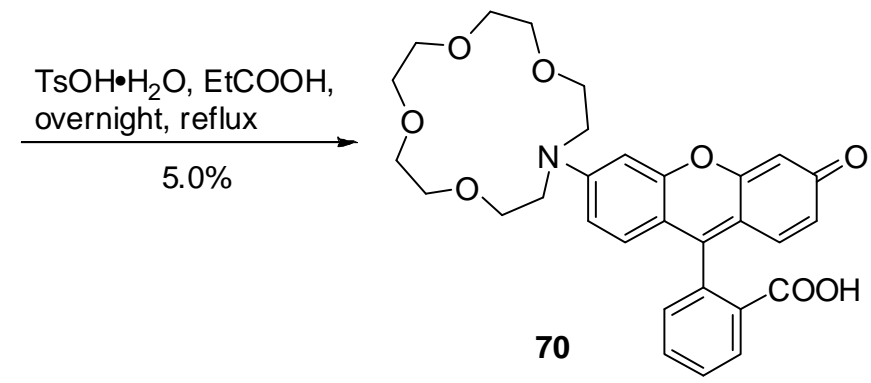

Scheme 24. Synthesis of the azacrown dyes - rhodols Ia-c and $\mathbf{7 0 .}$

The rhodols with crown-ether residues were synthesized under conditions mentioned in Section 2 of Chapter 2. The starting compounds $42 \mathbf{a}-\mathbf{c}, \mathbf{6 8}+\mathbf{6 9}$ were heated in propionic acid in the presence of catalytic amounts of $\mathrm{TsOH} \cdot \mathrm{H}_{2} \mathrm{O}$. The yields of the final compounds Ia-c and 70 were low, but we always isolated enough material to study the spectroscopic properties and parameters of the binding with calcium.

\subsection{Spectroscopic properties of the target compounds in the presence of $\mathrm{Ca}^{2+}$ ions}

The indicator 70 was prepared in order to check our assumption mentioned in Section 1 of Chapter 2. We assumed if we connect the crown ether with the aromatic ring in xanthene via only one heteroatom (nitrogen), such indicator will have a lower fluorescent response in the presence of calcium in the medium, than in the case of two common heteroatoms. The spectroscopic parameters of compound $\mathbf{7 0}$ were measured in methanolic and aqueous solutions (at $\mathrm{pH}=7.2$ ). 


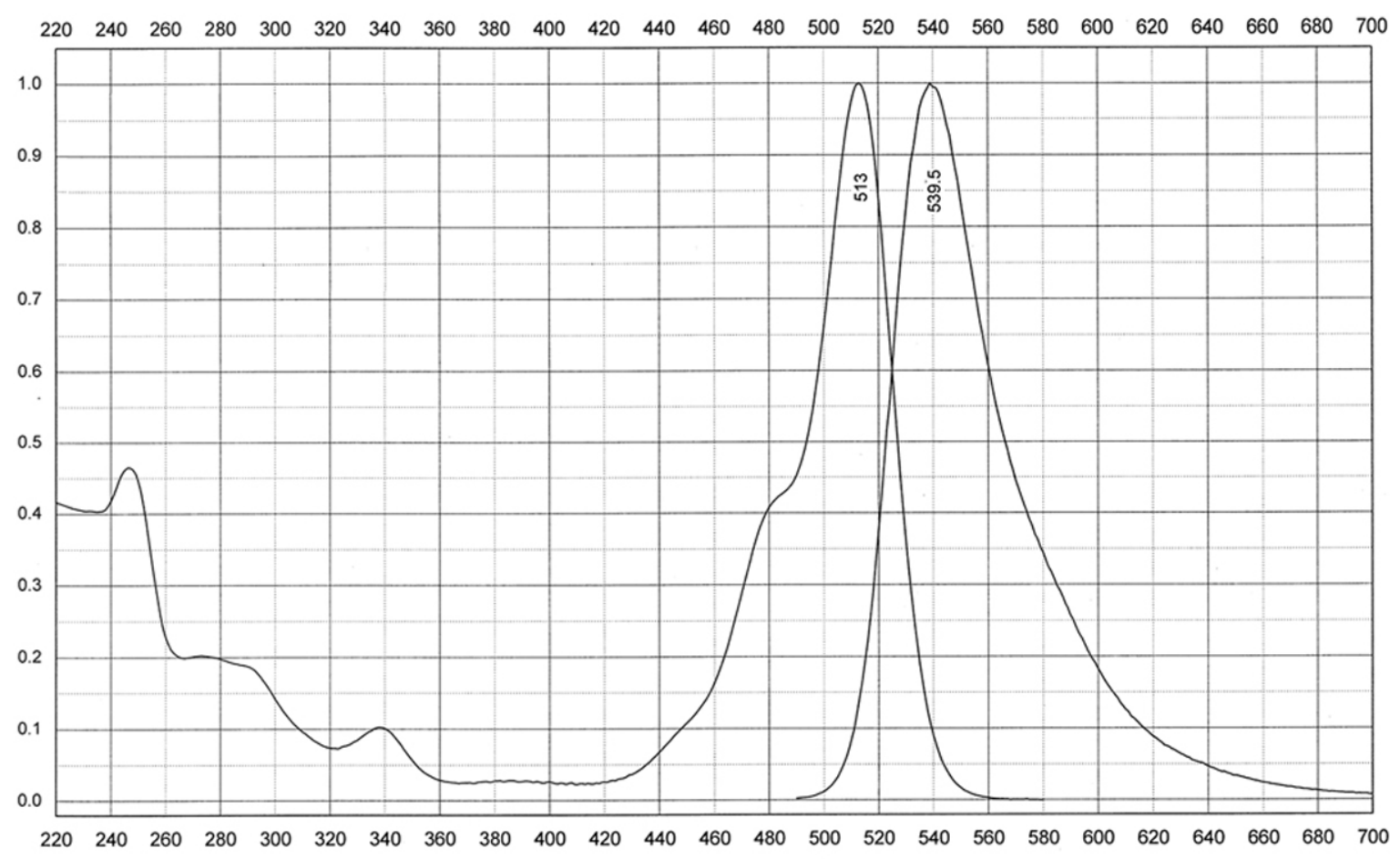

Figure 45. The absorption and fluorescence spectra of compound 70 in $\mathrm{MeOH}\left(\mathrm{c}=1.05 \times 10^{-5}\right.$ $\mathrm{M}): \lambda_{a b s}^{\max }=513 \mathrm{~nm}, \lambda_{f l}^{\max }=540 \mathrm{~nm}$.

The positions of absorption and fluorescence maxima only slightly deviate from the published values for the parent rhodol 39-H (in methanol: $\lambda_{a b s}^{\max }=517 \mathrm{~nm}, \lambda_{f l}^{\max }=542 \mathrm{~nm}$ ). ${ }^{[105]}$ The extinction coefficient for compound $\mathbf{7 0}$ was 1.2 times higher than that of rhodol 39-H $\left(5.2 \times 10^{4}\right.$ vs. $\left.3.9 \times 10^{4}\right)$. The fluorescence quantum yield for indicator $\mathbf{7 0}$ was also higher than that of analogue $39-\mathrm{H}(80 \%$ vs. $58 \%$ ). Thus, the chromophore of compound $\mathbf{7 0}$ was found to have good spectroscopic properties.

It is not surprising, that the absorption and fluorescence spectra of rhodols are sensitive to pH. In our case, such a dependence was also observed (Figure 46). 

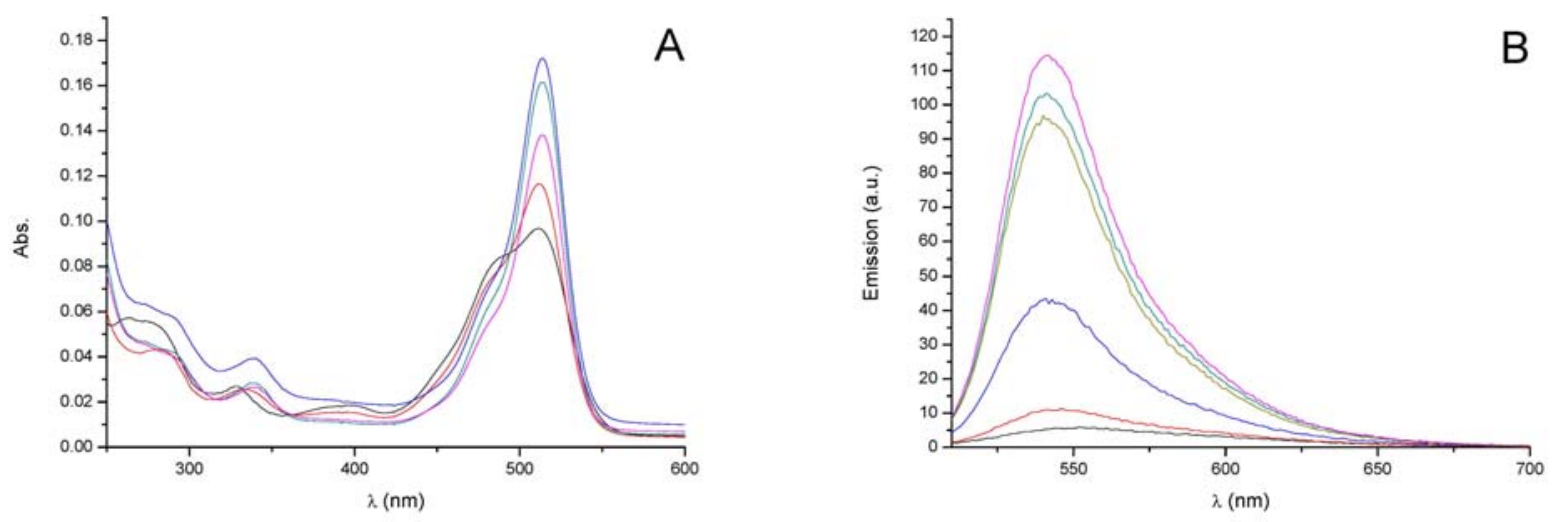

Figure 46. The absorption (left) and fluorescence (right) spectra of compound $70(\mathrm{C}=3.3$ $\mu \mathrm{M})$ at various $\mathrm{pH}$ values aqueous buffers: A) $\mathrm{pH}=2$ (black line), 5 (red), 7.6 (blue), 10 (green), 12 (light violet); B) pH = 2 (black line), 4 (red), 5 (blue), 6 (marine blue), 7.6 (light violet), 12 (olive green).

The observed sharp increase in the extinction coefficient of the absorption (up to 2 times) and in the fluorescence (up to 22 times) spectra upon changing of the $\mathrm{pH}$ value from 2 to 7.6 can be attributed to a deprotonation of the ammonium salt 70a and conversion of the indicator into the neutral form $\mathbf{7 0 b}$, which has to be significantly more fluorescent than the charged form 70a. Further basification of the medium (up to 12) causes some decrease in the intensity of the absorption and fluorescence bands which, probably, may be attributed to the formation of the colorless spirolactone 70c.
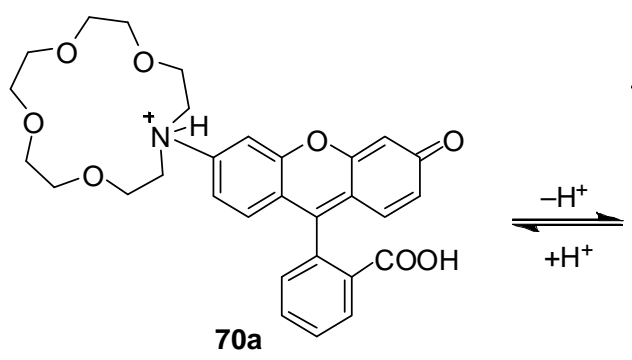<smiles>O=C(O)c1ccccc1-c1c2ccc(=O)cc-2oc2cc(N3CCOCCOCCOCCOCC3)ccc12</smiles>

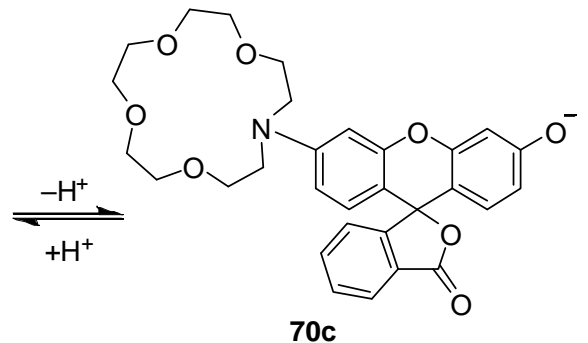<smiles></smiles>

Figure 47. The acid-base equilibria of the indicator $\mathbf{7 0}$ in aqueous solutions at various $\mathrm{pH}$ values. 
One of the advantages of compound $\mathbf{7 0}$ is the negligible change of the intensity of the fluorescent signal in the $\mathrm{pH}$ range (7-8) (see Figure 48).

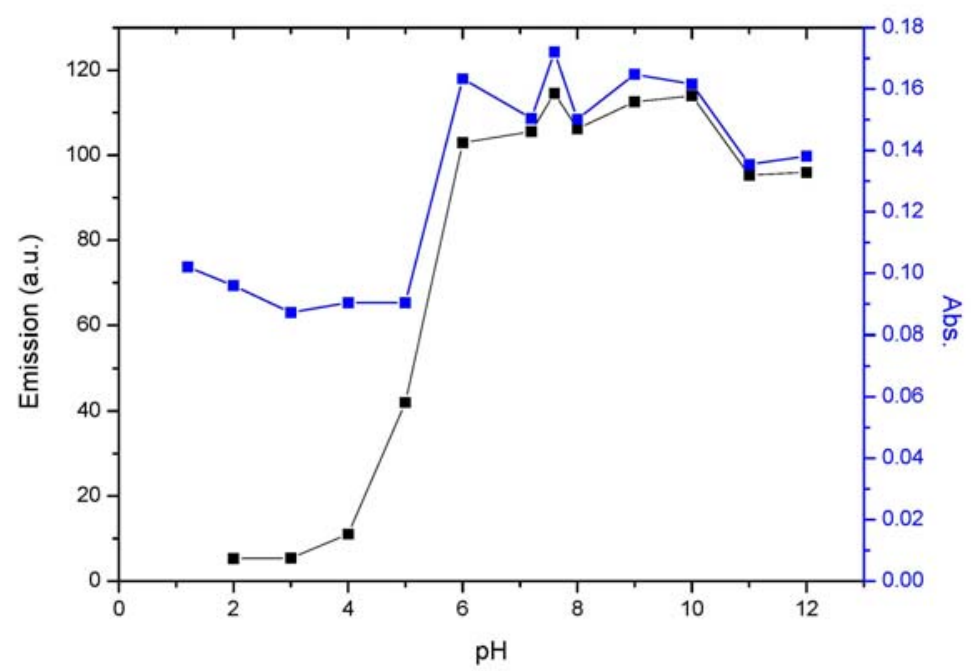

Figure 48. Dependence of the intensity of the absorption maximum (at $514 \mathrm{~nm}$ ) of $\mathbf{7 0}$ on the $\mathrm{pH}$ (blue curve), and the intensity of the fluorescence maximum (at $542 \mathrm{~nm}$ ) on the $\mathrm{pH}$ (black curve).

The main enhancement of the signals (absorption and fluorescence) is observed in the region from $\mathrm{pH}=4$ to $\mathrm{pH}=7$. From $\mathrm{pH} 10$ to 12 , a decrease in the fluorescence signal intensity was observed.
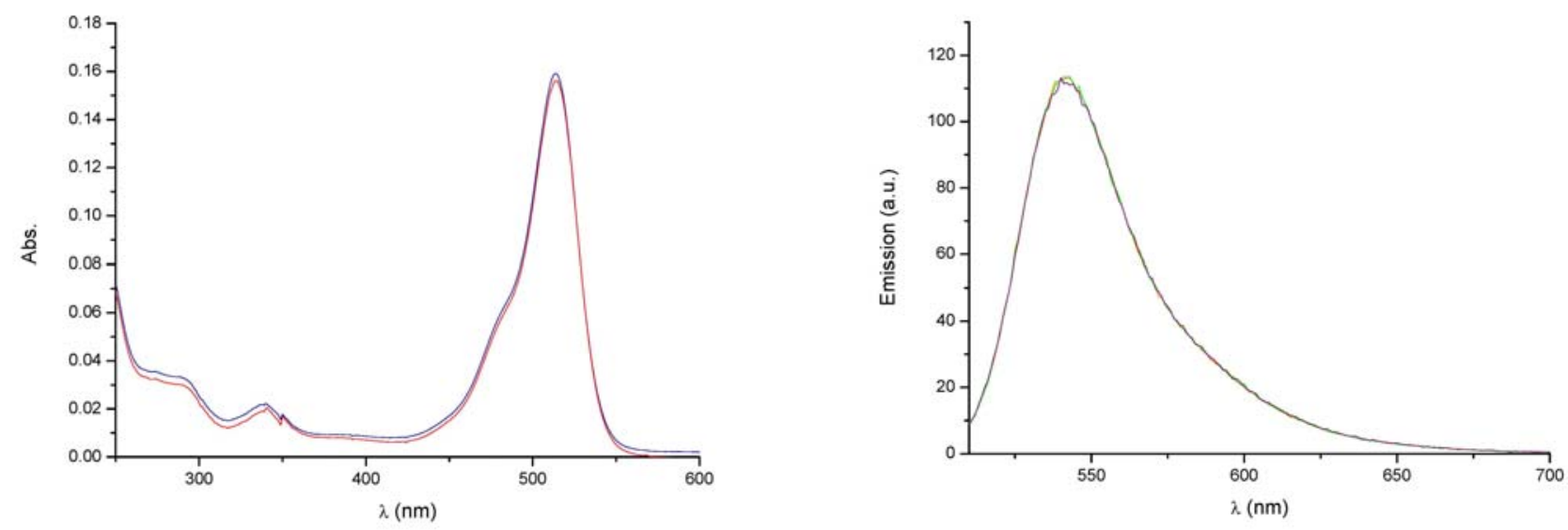

Figure 49. Changes in the absorption (left) and fluorescence (right) spectra of compound 70 (3.6 $\mu \mathrm{M}$ in an aqueous buffer solution at $\mathrm{pH}=7.2$ ) with $\left[\mathrm{Ca}^{2+}\right]=0-10^{-1} \mathrm{M}$. Curves at various concentration of $\left[\mathrm{Ca}^{2+}\right]$ are marked in different colors: absorption (blue line $-0 \mathrm{M}$, red line $100 \mathrm{mM}$ ); emission (orange line $-0 \mathrm{M}$, green line $-1 \mu \mathrm{M}$, purple line $-100 \mathrm{mM}$ ).

As we remember, in agreement with our expectations in the Section 1 of Chapter 2, the increase in calcium concentration should cause the only low increase in the intensity (less 
than $50 \%$ ) of the absorption and fluorescence bands. However, we did not observe any detectable changes in the spectra (Figure 49), that confirms our assumption to a greater extent.

The first of the target compounds - dye Ia - also has the positions of the absorption and fluorescence maxima very close to the published values for the rhodol 39-H $\left(\lambda_{a b s}^{\max }=517\right.$ $\mathrm{nm}, \lambda_{f l}^{\max }=542 \mathrm{~nm}$ in $\left.\mathrm{MeOH}\right){ }^{[105]}$

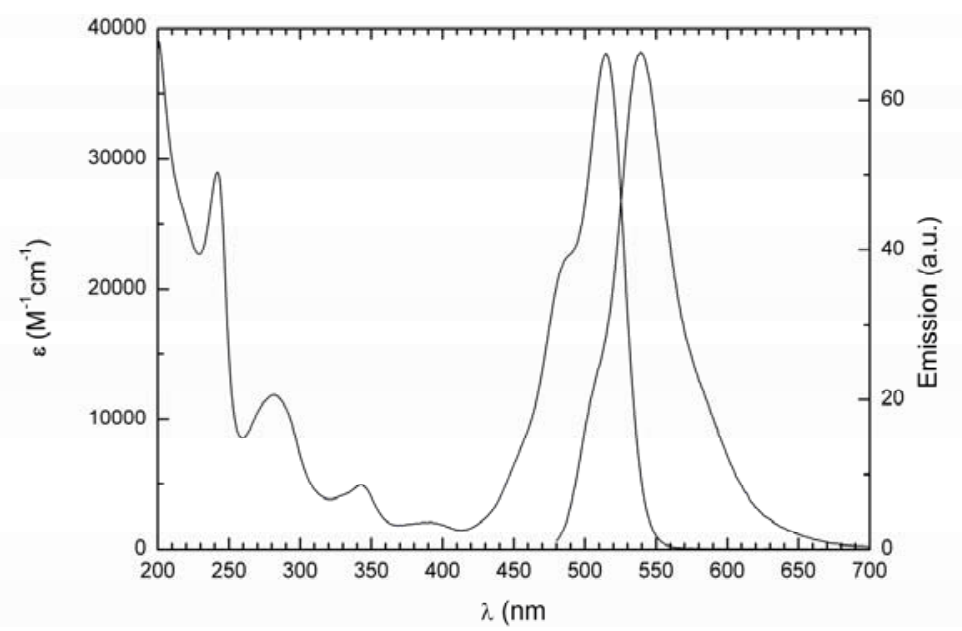

Figure 50. Absorption and fluorescence spectra of compound Ia in $\mathrm{MeOH}: \lambda_{a b s}^{\max }=515$ $\mathrm{nm}, \lambda_{f l}^{\max }=539 \mathrm{~nm}$.

Similar positions of the spectral maxima of compound 39-H and compound Ia can be attributed to a compensation of the introduction of the donor-alkoxy group into the $o$-position of the amino group and the absence of the second alkyl substituent on the nitrogen atom.

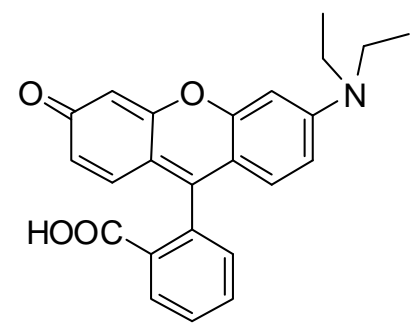

$39-\mathrm{H}$<smiles>O=C(O)c1ccccc1-c1c2ccc(=O)cc-2oc2cc(NCCOCCOCCOCCO)c(NCI)cc12</smiles>

la

Figure 51. Comparison of the structures of rhodol 39-H and compound Ia (the new structural elements are marked in red, the absent ethyl group is indicated with dashed lines).

The extinction coefficient for compound Ia was found to be similar to that for compound 39-H (39000 vs. $38000 \mathrm{M}^{-1} \mathrm{~cm}^{-1}$ ). The fluorescence quantum yield for indicator Ia was found to be higher than that for compound $39-\mathrm{H}$ (96\% vs. 58\%), due to the lower mobility of 
substituents on the nitrogen atom. The fluorescence quantum yield for indicator Ia was found to be higher than that for the crown rhodol 70 ( $96 \%$ vs. $80 \%$ ), due to a higher degree of conjugative interaction between the lone electron pair on the nitrogen atom with the xanthene $\pi$-system. Thus, the chromophoric part of compound Ia possesses good spectrophysical properties.
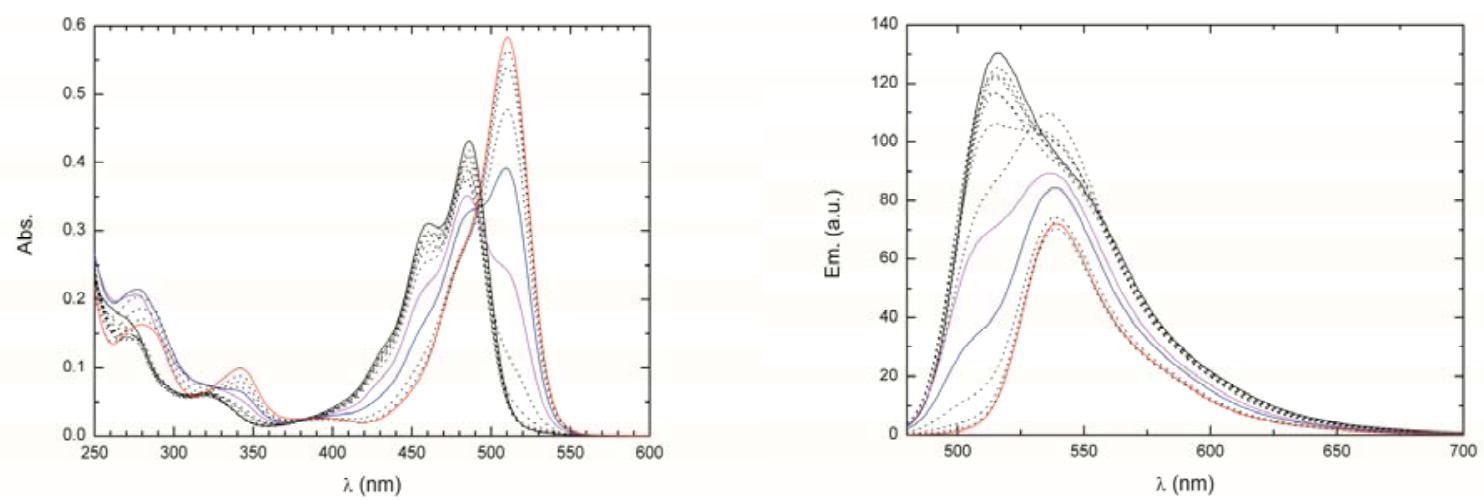

Figure 52. Changes in the absorption (left) and fluorescence (right) spectra of compound Ia $(\mathrm{C}=15 \mu \mathrm{M})$ at various $\mathrm{pH}$ values in aqueous buffer solutions: black line $-\mathrm{pH}=1.5$, violet line $-\mathrm{pH}=5.5$, blue line $-\mathrm{pH}=6$, red line $-\mathrm{pH}=8$.

The absorption spectra of aqueous solutions of compound $\mathbf{I a}$ at $\mathrm{pH}=1.5-5$ show two bands at 458 and at $485 \mathrm{~nm}$. The first band can be attributed to the form Ia' (Scheme 25) with the protonated nitrogen atom. The second band must be attributed to the form Ia" with a protonated oxygen atom. The absorption spectrum at $\mathrm{pH} 5.5$ has only one maximum at the $485 \mathrm{~nm}$, which may be attributed to the form Ia". Two shoulders occur at 460 and $509 \mathrm{~nm}$ are the residual signals contributed by the forms $\mathbf{I a}^{\prime}$ and form $\mathbf{~} \mathbf{a}^{\sim}$ (neutral). A further increase in the $\mathrm{pH}$ (up to $\mathrm{pH}=6$ ) is accompanied by the disappearance of the shoulder at $460 \mathrm{~nm}$ (absence of the form $\mathbf{I a}^{\prime}$ ). At $\mathrm{pH}=6$, the signal at $509 \mathrm{~nm}$ becomes the most intensive one (maximum), and the signal at $485 \mathrm{~nm}$ decreases and is registered only as a shoulder. In basic solution ( $\mathrm{pH}=8)$, the signals from the forms $\mathbf{I a}^{\prime \prime}$ (at $485 \mathrm{~nm}$ ) and $\mathbf{I a}^{\prime}$ disappear, and the signal from the form $\mathbf{I a}^{\sim}$ increases. Probably the zwitter-ionic form $\mathbf{I a}^{\sim}$ and the colorless spirolactone Ia** are less stable forms of compound Ia under neutral and basic conditions. 
<smiles>O=C(O)c1ccccc1-c1c2ccc(=O)cc-2oc2cc([NH2+]CCOCCOCCOCCO)c(O)cc12</smiles><smiles>[CH]=C</smiles><smiles>O=C(O)c1ccccc1-c1c2cc(OCCOCCOCCOCC[18F])c(=[NH+]CCO)cc-2oc2cc(O)ccc12</smiles><smiles></smiles>

la<smiles>[CH]=C</smiles><smiles>O=C([O-])c1ccccc1-c1c2cc(OCCOCCOCCOCC[18F])c(=[NH+]CCO)cc-2oc2cc(O)ccc12</smiles><smiles></smiles>

$1 a^{*}$<smiles>C=CC1CC12Oc1cc([O-])ccc1C21OC(=O)c2ccccc21</smiles>

$1 a^{* *}$

Scheme 25. Acid-base equilibria of the indicator Ia in aqueous solutions.

In the fluorescence spectrum of Ia recorded at $\mathrm{pH}=1.5$, only one emission band at 516 $\mathrm{nm}$ (with a shoulder at $550 \mathrm{~nm}$ ) is observed. The band at $516 \mathrm{~nm}$ is probably a superposition of the signals from two the forms Ia' and Ia", and the shoulder at $550 \mathrm{~nm}$ can be attributed to the form $\mathbf{I a}$. At $\mathrm{pH}=5.5$, the shoulder at $550 \mathrm{~nm}$ transforms into a maximum (at $538 \mathrm{~nm}$ ),

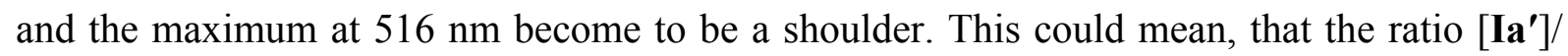
[Ia"] in decreases solution. At $\mathrm{pH}=6$, the intensity of the shoulder (at $516 \mathrm{~nm}$ ) decreases considerably, while the intensity of the maximum (at $538 \mathrm{~nm}$ ) decreases only slightly. In basic solution (at $\mathrm{pH}=8$ ) the intensity of the band at $538 \mathrm{~nm}$ becomes slightly lower, while the shoulder at $516 \mathrm{~nm}$ disappears.

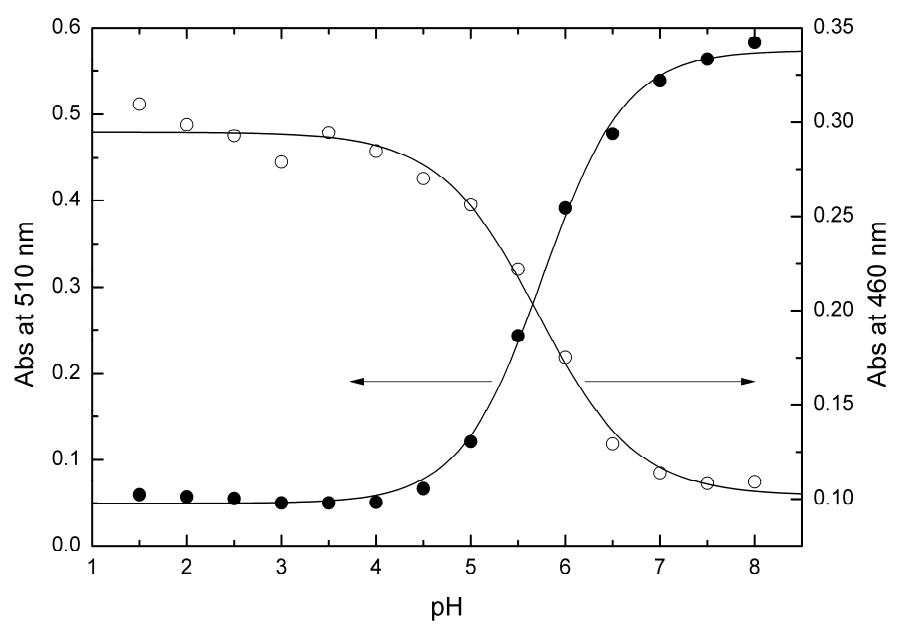

Figure 53. Absorption of a solution of Ia at $510 \mathrm{~nm}$ (filled symbols, left axis) and at $460 \mathrm{~nm}$ (hollow symbols, right axis) plotted as a function of the $\mathrm{pH}$ value. 
The same feature as for compound $\mathbf{7 0}$ - the negligible change of absorption upon variation of the $\mathrm{pH}$ value in the range between 7 and 8 - was found for compound Ia. It is obvious, if we represent the data on the absorption as given in Figure 53. The main enhancement of the signal (absorption) is registered in the $\mathrm{pH}$ range from 4 to 7 . The changes in absorption in the interval of $\mathrm{pH} \mathrm{7-8}$ are negligible for the band at $460 \mathrm{~nm}$, while the intensity of the band at $510 \mathrm{~nm}$ increases by increasing the basicity of the solution.
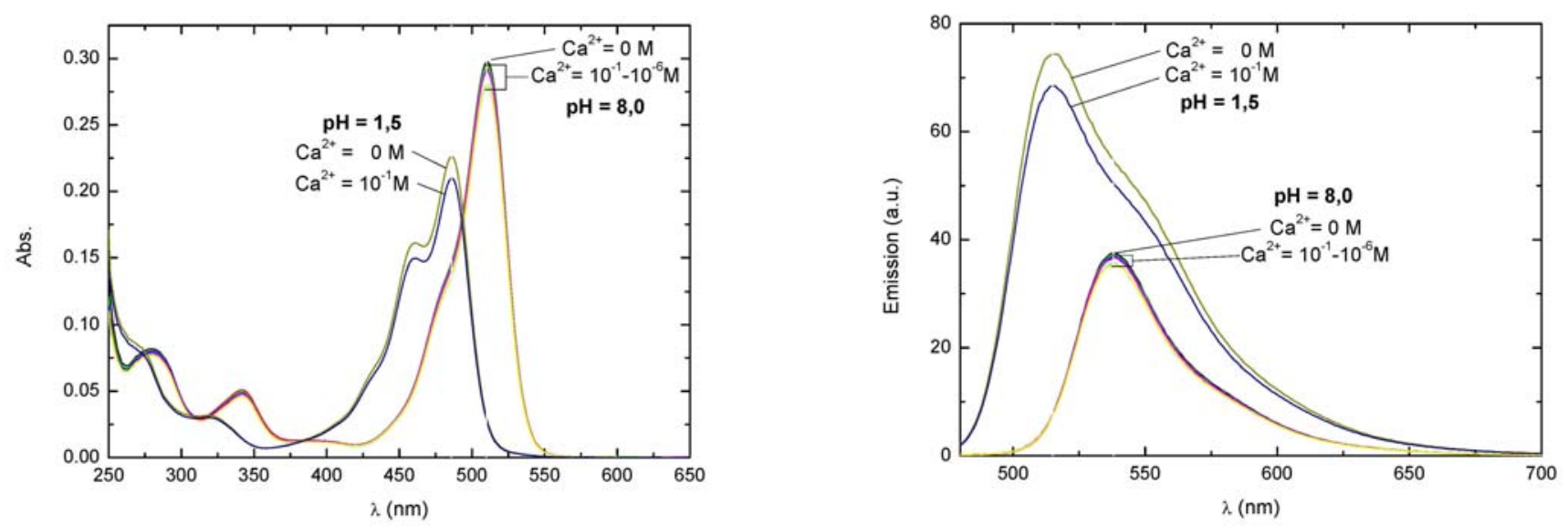

Figure 54. Changes in the absorption (left) and fluorescence (right) spectra of compound Ia $(\mathrm{C}=7.5 \mu \mathrm{M})$ in aqueous buffer solutions $(\mathrm{pH}=1.5,8.0)$ with free $\left[\mathrm{Ca}^{2+}\right]$ ranging from 0 to $10^{-1} \mathrm{M}$. Lines of various colors correspond to various Ca-concentration: $\mathbf{p H}=\mathbf{1 . 5}$ : absorption and emission (ochre line $-0 \mathrm{M}$, blue line $-100 \mathrm{mM}$ ); $\mathbf{p H}=\mathbf{8 . 0}$ : absorption and emission: the set of color lines represents negligible changes of the spectra in the range of calcium concentrations from $0 \mathrm{M}$ to $100 \mathrm{mM}$.

Variation of the calcium concentration in the interval from 0 to $0.1 \mathrm{M}$ by one-order of magnitude steps $\left(0,10^{-6}, 10^{-5}, 10^{-4}\right.$ etc.) in slightly alkaline solutions did not show any significant changes in the absorption and fluorescence spectra of compound Ia (absorption band at $510 \mathrm{~nm}$ in the left and fluorescence band at $538 \mathrm{~nm}$ in the right picture). Another result was obtained at a low $\mathrm{pH}$ value (1.5): we observed some decrease in the intensity of the absorption $(486 \mathrm{~nm})$ and fluorescence $(515 \mathrm{~nm})$ bands upon increasing $\left[\mathrm{Ca}^{2+}\right]$ values. Thus, we cannot recommend to use compound Ia as a calcium sensor, because the effects were observed only at $\mathrm{pH}=1.5$ (which is far from physiological values). To explain the low effect, it is necessary to analyze the spatial structure of compound Ia. In the initial state, the hydrogen atom of the NH group is most probably located in the crown ether cavity and forms an H-bond with the oxygen atoms. In order to bind a calcium cation, the crown-ether ring needs to be transformed in such a way that the lone electron pair of nitrogen is directed into 
the cavity, and the hydrogen atom turns to be outside the cavity. ${ }^{[88]}$ Probably, this process is unfavorable for compound Ia, and it does not coordinate the calcium cation at all.

The main feature, which influences the spectroscopic properties of the next indicator Ib, is the presence of an additional ethyl group on the nitrogen atom. As a result, this donor group shifts the positions of the absorption and the emission maxima of compound Ib to the red (by $17-19 \mathrm{~nm}$ ), in comparison with the corresponding values for compound Ia ( $\lambda_{a b s}^{\max }=515$ $\mathrm{nm}, \lambda_{f l}^{\max }=539 \mathrm{~nm}$ for Ia vs. $\lambda_{a b s}^{\max }=542 \mathrm{~nm}, \lambda_{f l}^{\max }=558 \mathrm{~nm}$ for Ib).

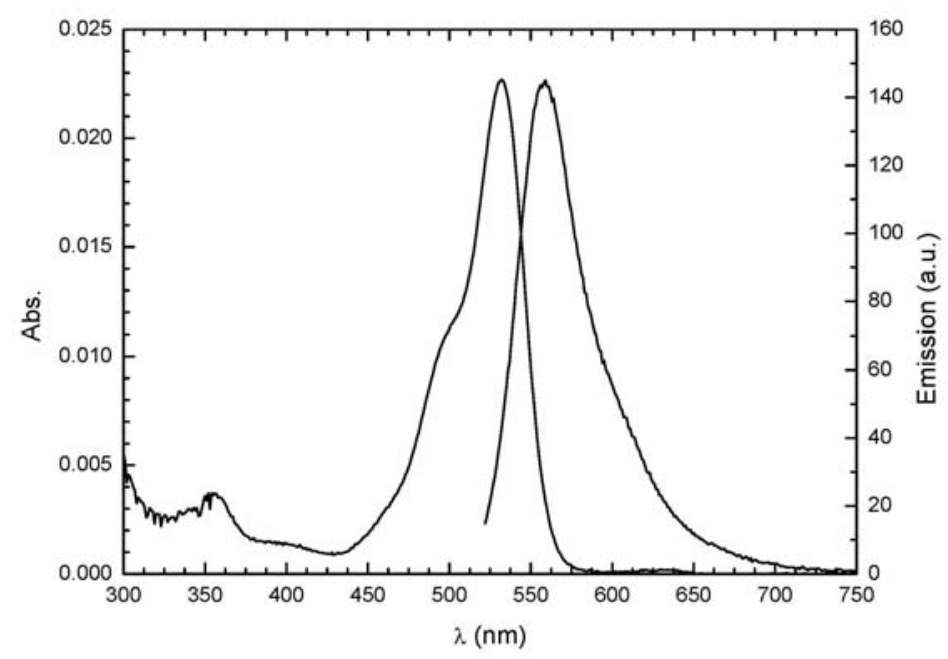

Figure 55. Absorption and fluorescence spectra of compound Ib in $\mathrm{MeOH}: \lambda_{a b s}^{\max }=542 \mathrm{~nm}(\varepsilon$ $\left.=22300 \mathrm{M}^{-1} \mathrm{~cm}^{-1}\right), \lambda_{f l}^{\max }=558 \mathrm{~nm}$.

The second consequence is a decrease of the extinction coefficient for compound Ib up to 2 times in comparison with the same parameter for compound Ia. Another structural feature which results due to the additional ethyl group is a rotation of the dialkylamino group out of its coplanarity with the xanthene aromatic system. A direct consequence of the absence of coplanarity is a decrease in the conjugation between the nitrogen lone pair and the aromatic $\pi$ system, and a decrease in the fluorescence quantum yield to $16 \%$. 

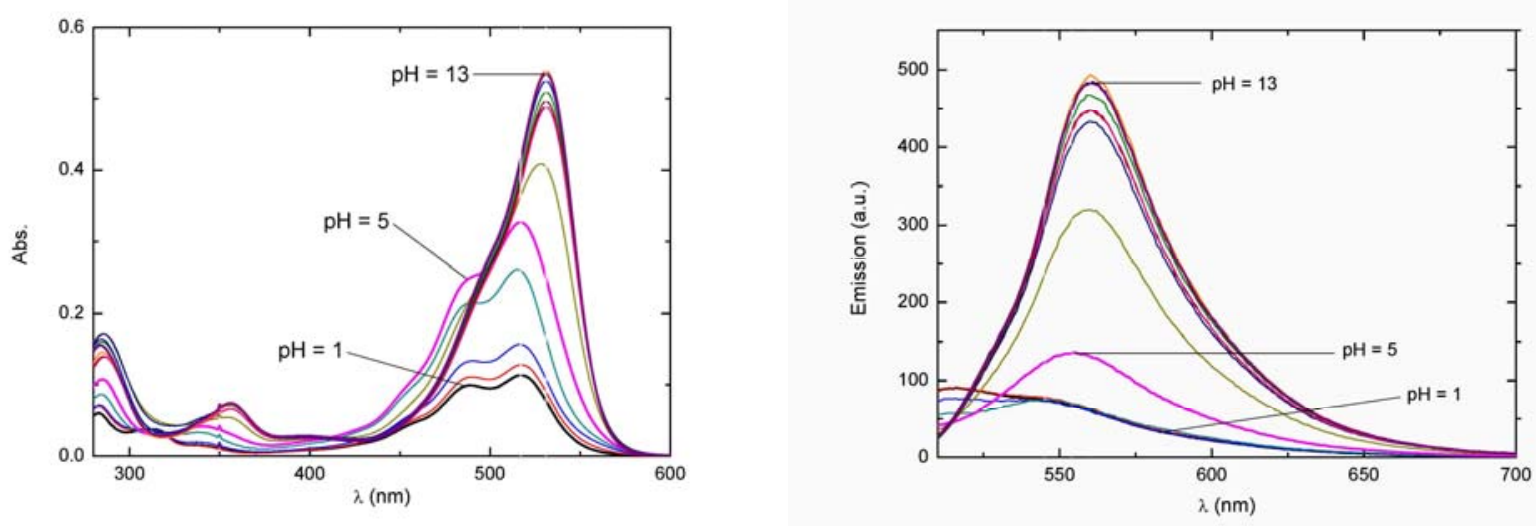

Figure 56. Changes in absorption (left) and fluorescence (right) spectra of compound $\mathbf{I b}(\mathrm{C}=$ $17 \mu \mathrm{M})$ at various $\mathrm{pH}$ values of the aqueous buffer solutions; black line: $\mathrm{pH}=1$, pink line: $\mathrm{pH}$ $=5$, violet line: $\mathrm{pH}=13$.

The absorption spectra of aqueous solutions of compound $\mathbf{I b}$ in the range of $\mathrm{pH}$ from 1 to 5 (in acidic medium) contain two bands at $488 \mathrm{~nm}$ and at $517 \mathrm{~nm}$. The first band, fluoresceinlike, could be attributed to the form $\mathbf{I b}^{\prime}$ (Scheme 26) with a protonated nitrogen atom. The second band could be attributed to the form $\mathbf{I b}^{\prime \prime}$ with a protonated oxygen atom. At $\mathrm{pH}>5$, there is only one band at $531 \mathrm{~nm}$, which we attribute to the neutral form $\mathbf{I b}^{\sim}$ (and/or deprotonated form $\left.\mathbf{I b}^{*}\right)$. In the fluorescence spectra in the whole $\mathrm{pH}$ range only one emission band was observed. Its maximum shifted from $514 \mathrm{~nm}($ at $\mathrm{pH}=1)$ to $554 \mathrm{~nm}(\mathrm{pH}=5)$, and then to $560 \mathrm{~nm}(\mathrm{pH}=13)$.

The observed increase in absorbance (up to 3 times) and in the fluorescence intensity (up to 5 times) upon changes of the $\mathrm{pH}$ from 1 to 8 can be explained with the deprotonation of the ammonium salts $\mathbf{I b}^{\prime}$ and $\mathbf{I} \mathbf{b}^{\prime \prime}$ and the conversion of the indicator to the neutral form $\mathbf{I b}^{\sim}$, which is significantly more fluorescent than the cations $\mathbf{I b}^{\prime}$ and $\mathbf{I b}^{\prime \prime}$. Further basification of the solution (up to $\mathrm{pH}=13$ ) does not decrease the intensity of the absorption and fluorescence. Unlike in the case of compound 70, the colorless spirolactone (Ib**) is not formed in the basic medium. 
<smiles>CC[NH+](CC)c1cc2oc3cc(=O)ccc-3c(-c3ccccc3C(=O)O)c2cc1OCCOCCOCCOC</smiles><smiles>[CH]=C</smiles><smiles></smiles>
lb"

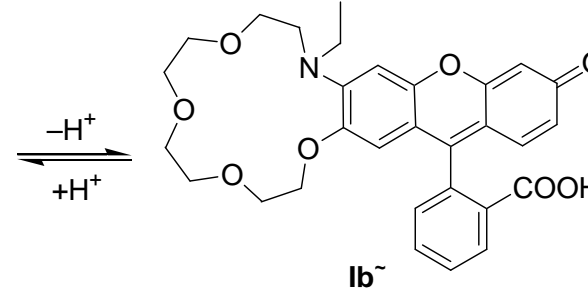<smiles>[CH]=C</smiles><smiles>CC[N+]1=C(OCCOCCOCCOCCO)C=C2Oc3cc(O)ccc3C(c3ccccc3C(=O)[O-])=C2C=C1c1ccccc1</smiles>

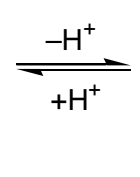

$<$<smiles>CCN1CCOCCOCCOCCOc2cc3c(-c4ccccc4C(=O)[O-])c4ccc(=O)cc-4oc3cc21</smiles>

Ib*<smiles>CCN1CCOCCOCCOCCOc2c(N3CCOCCO3)cc3c(c21)C1(OC3=O)c2ccccc2Oc2cc([O-])ccc21</smiles>

Ib**

Scheme 26. Protonated and deprotonated forms of the indicator Ib in aqueous solutions at various $\mathrm{pH}$ values.

The same feature as for compound Ia - a negligible change of the fluorescence and absorption signal at $\mathrm{pH}=7-8$ was also observed for compound $\mathbf{I b}$. This is demonstrated clearly by the representation the data in Figure 57.
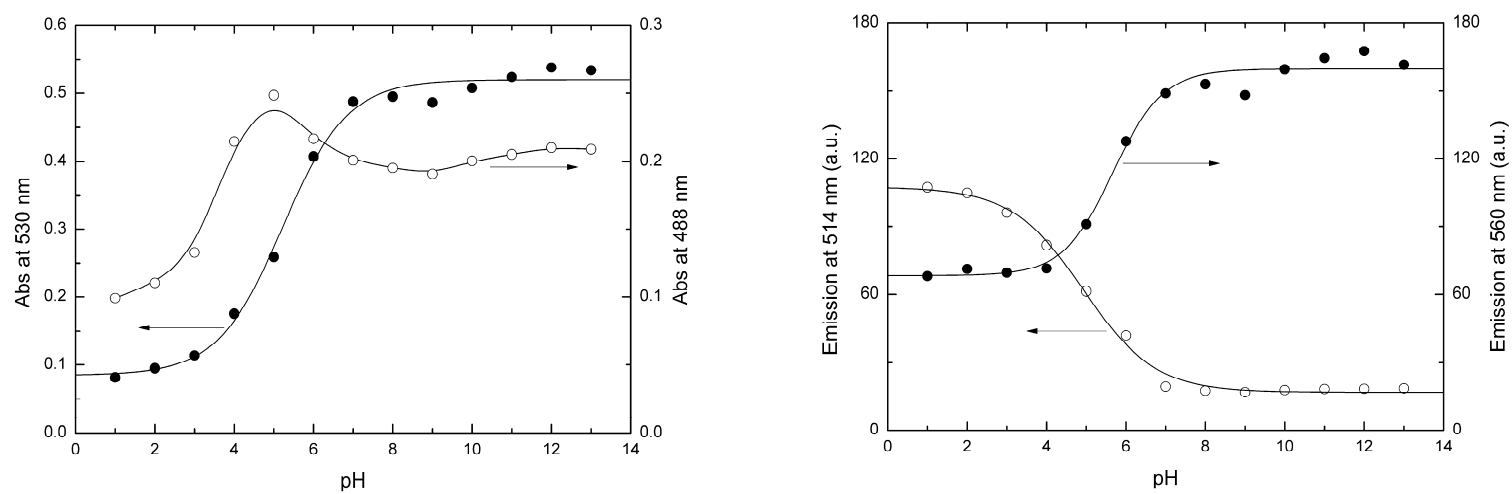

Figure 57. Absorption and emission intensities of the solutions of the compound Ib at various $\mathrm{pH}$ values. Left picture: absorption at $530 \mathrm{~nm}$ (filled symbols, left axis) and at 488 $\mathrm{nm}$ (hollow symbols, right axis) at $\mathrm{pH}=1-13$; right picture: emission at $560 \mathrm{~nm}$ (filled symbols, left axis) and at $514 \mathrm{~nm}$ (hollow symbols, right axis at $\mathrm{pH}=1-13$.

The main changes of the signal intensities (absorption and fluorescence) were observed at $\mathrm{pH}=3-7$; changes at $\mathrm{pH} 0-3$ and 7-14 were insignificant. 

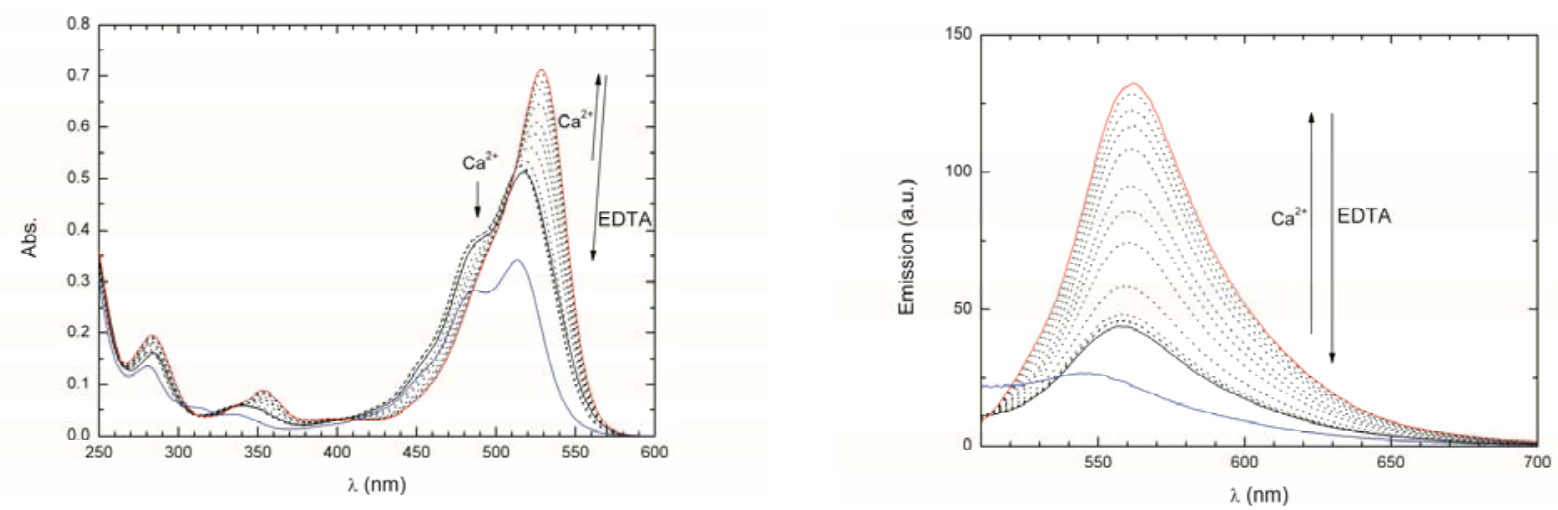

Figure 58. Changes in the absorption (left) and fluorescence (right) spectra of compound Ib $(\mathrm{C}=18 \mu \mathrm{M})$ in aqueous buffer solutions $(\mathrm{pH}=7.2)$ with free $\left[\mathrm{Ca}^{2+}\right]=0-0.1 \mathrm{M}$; the different concentration of $\left[\mathrm{Ca}^{2+}\right]$ are marked in different colors: absorption and emission (black line -0 M, dashed lines - intermediate concentrations, red line - $100 \mathrm{mM}$, blue line - after the addition of EDTA to a final concentration of $27 \mathrm{mM}$ ). The arrows indicate the changes observed with increasing concentrations of $\mathrm{Ca}^{2+}$ and EDTA.

In the absorption spectrum of the initial solution of compound Ib (without calcium), two bands were observed at 484 and $512 \mathrm{~nm}$ which may be attributed to the species $\mathbf{I b}^{\sim}$ and $\mathbf{I b}^{\sim}$ mentioned above. Only the first of these forms can interact with $\mathrm{Ca}^{2+}$ (due to the absence of the positively charged nitrogen atom).

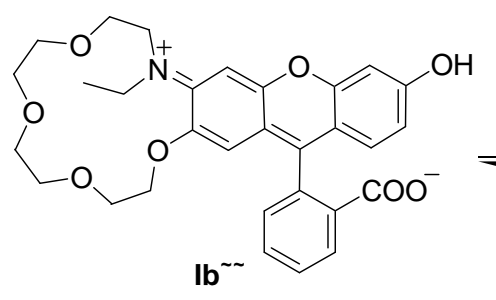<smiles>CCCOCCOCCOCCOCCOCCN(CC)c1cc2oc3cc(=O)ccc-3c(-c3ccccc3C(=O)O)c2cc1O</smiles>

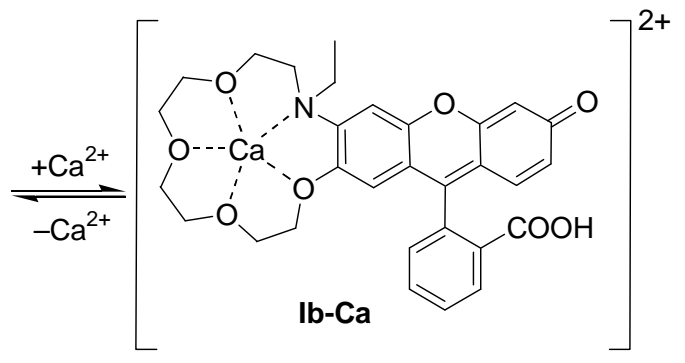

Scheme 27. Complexation of the calcium cation by indicator Ib.

An increase in the calcium ion concentration from 1 to $126 \mathrm{mM}$ resulted in a red shift of the absorption band (from 512 to $526 \mathrm{~nm}$ ), and simultaneously, an increase in the absorption intensity was observed (up to $40 \%$ at $\left[\mathrm{Ca}^{2+}\right]=126 \mathrm{mM}$ ). These changes of the calcium concentration increased the intensity of the fluorescence band: it became 3 times more intensive at $\left[\mathrm{Ca}^{2+}\right]=126 \mathrm{mM}$. However, the red shift of the fluorescence maximum (which was also observed) was found to be only $4 \mathrm{~nm}$ (from $558 \mathrm{~nm}$ in the absence of calcium to 562 $\mathrm{nm}$ at $\left.\left[\mathrm{Ca}^{2+}\right]=126 \mathrm{mM}\right)$. Such kind of atypical influence of calcium on absorption and fluorescence spectra of an indicator with the xanthene fluorophore (red shift) may be 
attributed to the fact that the calcium cation does not only coordinate to the nitrogen atom, but also to the oxygen atom attached to the aromatic ring.

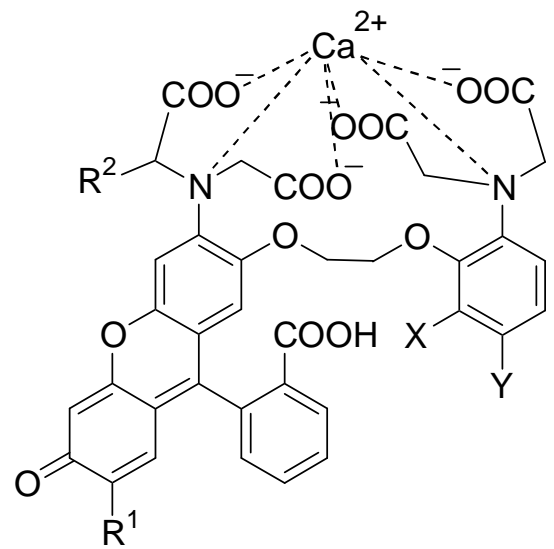

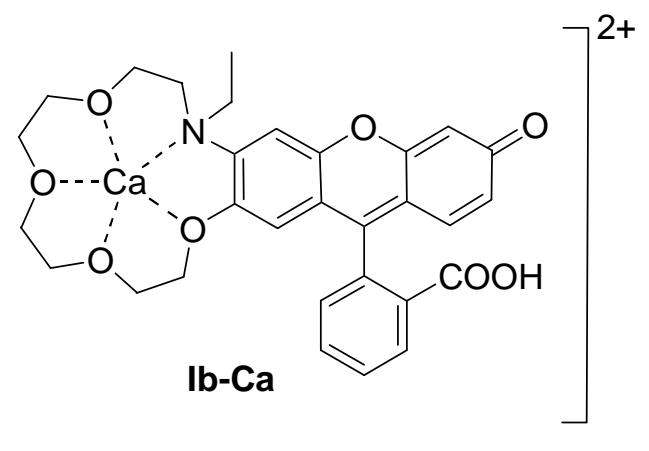

14c: FluoRhod-2: $\mathrm{R}^{1}=\mathrm{CH}_{2} \mathrm{CH}_{2} \mathrm{COOH}, \mathrm{R}^{2}=\mathrm{Y}=\mathrm{CH}_{3}, \mathrm{X}=\mathrm{H}$;

Figure 59. Comparison of the two calcium indicators with a rhodol fluorophore.

As was discussed in Chapter 1, the compound 14c with a rhodol fluorophore displayed a blue shift of the absorption and fluorescence maxima upon coordination with calcium (or protonation). The indicator Ib also displayed a blue spectral shift upon protonation. However, protons coordinate first with the nitrogen atoms. In the case of indicator $\mathbf{I b}$, the calcium cation does not only bind to the nitrogen, but also to an adjacent oxygen atom. In the case of an octadental ligand as in the indicator $\mathbf{1 4 c}$, the calcium cation is kept only by six heteroatoms. Therefore, it does not need to bind to the oxygen atom attached to the rhodol system.

As far as the quantitative parameters of compound $\mathbf{I b}$ are concerned, they can best be evaluated, when the data are presented as in the Figure 60.
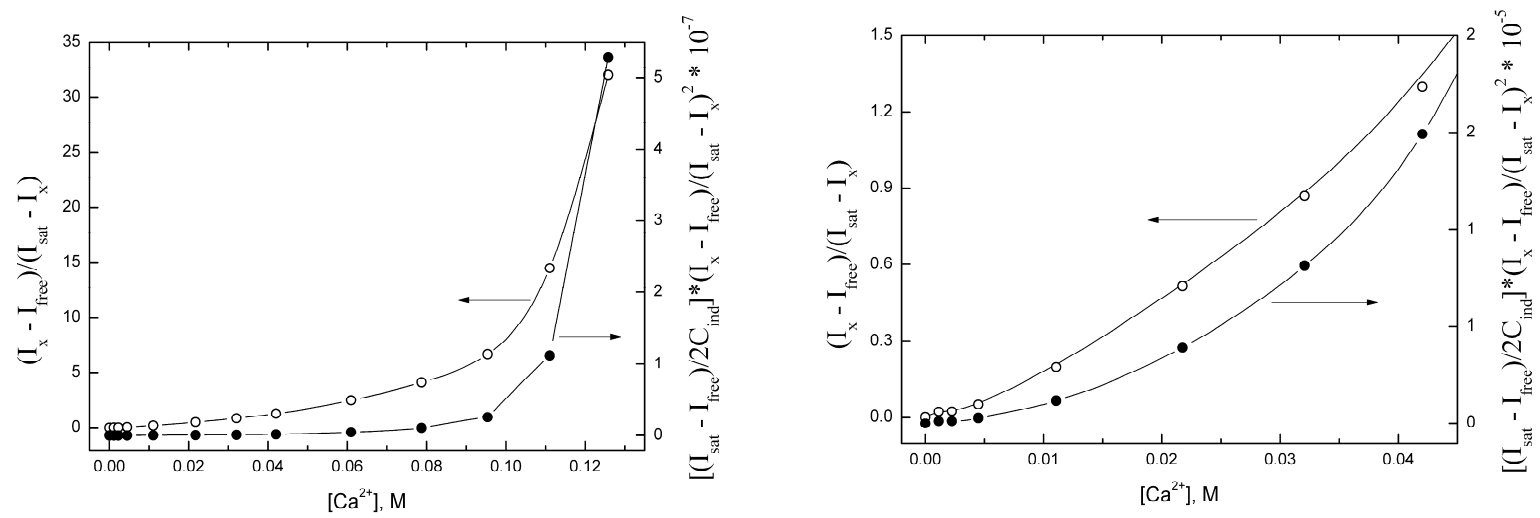

Figure 60. Dependence of the fluorescence parameters on the calcium concentration. Left plot: left vertical axis $\frac{I_{x}^{f l}-I_{\text {free }}^{f l}}{I_{\text {sat }}^{f l}-I_{x}^{f l}}$ (for the $1: 1$ complex of $\mathbf{I b}$ with $\mathrm{Ca}^{2+}$; hollow circles), right 
vertical axis $\frac{\left(I_{x}^{f l}-I_{\text {free }}^{f l}\right) *\left(I_{\text {sat }}^{f l}-I_{\text {free }}^{f l}\right)}{\left(I_{\text {satl }}^{f l}-I_{x}^{f l}\right)^{2}} * \frac{1}{2 C_{\text {ind }}}$ (for the $2: 1$ complex of Ib with $\mathrm{Ca}^{2+}$; filled circles). $C_{\text {ind }}$ stands for the whole indicator concentration in solution; for other abbreviations, see Section 2.1. The right plot represents the same parameters in the range of $\left[\mathrm{Ca}^{2+}\right]=0.000$ $0.042 \mathrm{M}$.

As was mentioned in Section 2.1, the calcium concentration from the fluorimetric data can be calculated according to the equation (3):

$$
\left[C a^{2+}\right]=K_{D}\left(\frac{I_{x}^{f l}-I_{f r e e}^{f l}}{I_{\text {sat }}^{f l}-I_{x}^{f l}}\right)
$$

The unknown parameter $K_{\mathrm{D}}$ may be easily calculated from the plot $\frac{I_{x}^{f l}-I_{\text {free }}^{f l}}{I_{\text {sat }}^{f l}-I_{x}^{f l}}$ vs. $\left[\mathrm{Ca}^{2+}\right]$. The tangent of this curve is equal to $K_{D}^{-1}$, if the dependence is linear. In our case, the resulting curve (hollow circles) is not linear; the most probable explanation is a false binding model. The equation (3) is valid for a $1: 1$ complex of the indicator with calcium. On the basis of the structure of compound $\mathbf{I b}$, we can assume the formation of a sandwich-like complex with two molecules of the indicator and one calcium ion. For this model, another equation for the calcium concentration (and dissociation constant) is obtained:

$$
\left[C a^{2+}\right]=K_{D} \frac{\left(I_{x}^{f l}-I_{\text {free }}^{f l}\right) *\left(I_{\text {sat }}^{f l}-I_{\text {free }}^{f l}\right)}{\left(I_{\text {satl }}^{f l}-I_{x}^{f l}\right)^{2}} * \frac{1}{2 C_{\text {ind }}}
$$

However, even with this case a linear dependence of $\frac{\left(I_{x}^{f l}-I_{\text {free }}^{f l}\right) *\left(I_{\text {sat }}^{f l}-I_{\text {free }}^{f l}\right)}{\left(I_{\text {satl }}^{f l}-I_{x}^{f l}\right)^{2}} * \frac{1}{2 C_{\text {ind }}}$ vs. $\left[\mathrm{Ca}^{2+}\right]$ was not observed, and therefore, the dissociation constant for the complex of compound Ib and calcium cannot be calculated. The absence of a linear dependence for both models (1:1 and 2:1 complexes) may be explained, for example, by the presence of both complexes in an equilibrium (Scheme 28). In this case, however, we cannot evaluate any quantitative parameters, because we cannot extract the coefficient " $\mathrm{c}$ " in the dependence $I_{f l}=$ $\mathrm{c}^{*}[\mathrm{IM}]$ from the fluorescence measurements.

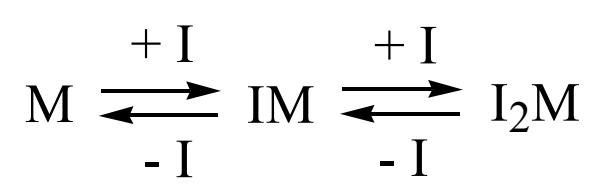


Scheme 28. The equilibrium in the system indicator - metal ion, in which I stands for indicator molecule, $\mathrm{M}$ for metal ion.

Thus, we cannot calculate the equilibrium constants for complexes $I M$ and $I_{2} M$, and this complicates the comparison of the binding properties of compound Ib with those of the other known indicators. Moreover, the inavailability of the binding constants makes the calculation of the calcium concentrations difficult. However, we can conclude that for compound Ib the "working interval" of calcium concentrations is from 5 to $126 \mathrm{mM}$.

The change of the donor ethyl group in compound Ib to a carboxymethyl residue results in the compound Ic with nearly the same positions of the absorption and emission maxima, as for the unsubstituted indicator Ia.

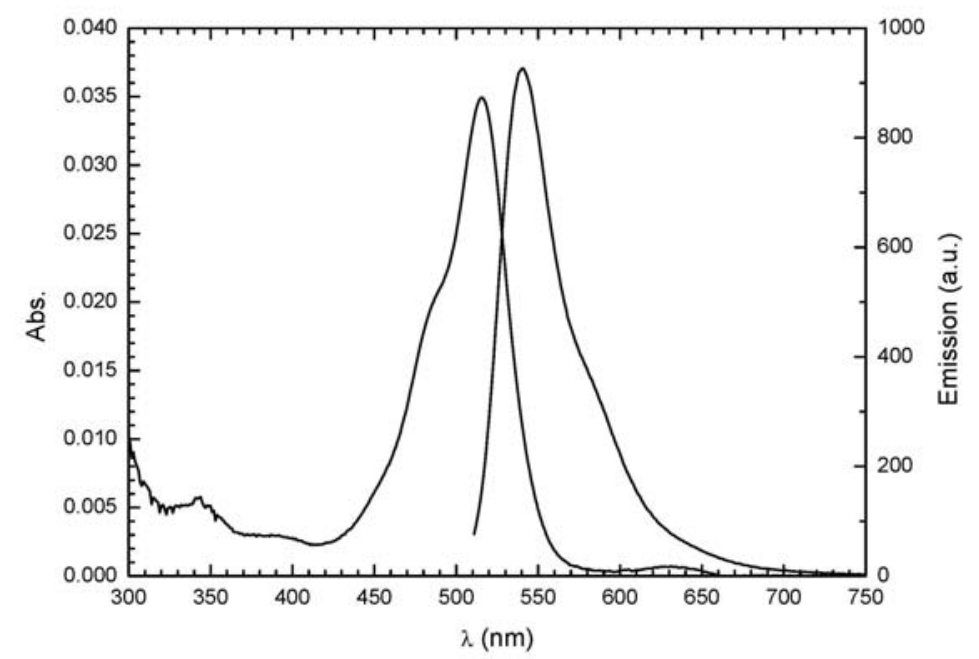

Figure 61. Absorption and fluorescence spectra of compound Ic in methanol: $\lambda_{a b s}^{\max }=517 \mathrm{~nm}$ $\left(\varepsilon=30300 \mathrm{M}^{-1} \mathrm{~cm}^{-1}\right), \lambda_{f l}^{\max }=541 \mathrm{~nm}$.

The extinction coefficient of compound Ic is slightly lower than that of compound Ia (30300 vs. $39000 \mathrm{M}^{-1} \mathrm{~cm}^{-1}$ ); at the same time, the $\varepsilon$-value for Ic is 1.5 times higher than that of compound Ib. As a result of the introduction of the carboxymethyl group, the coplanarity of the dialkylamino group with the xanthene aromatic system may be distorted as in the case of compound Ib). However, for the compound Ic, the fluorescence quantum yield was found to be high ( $72 \%$ vs. $16 \%$ for compound Ib). 

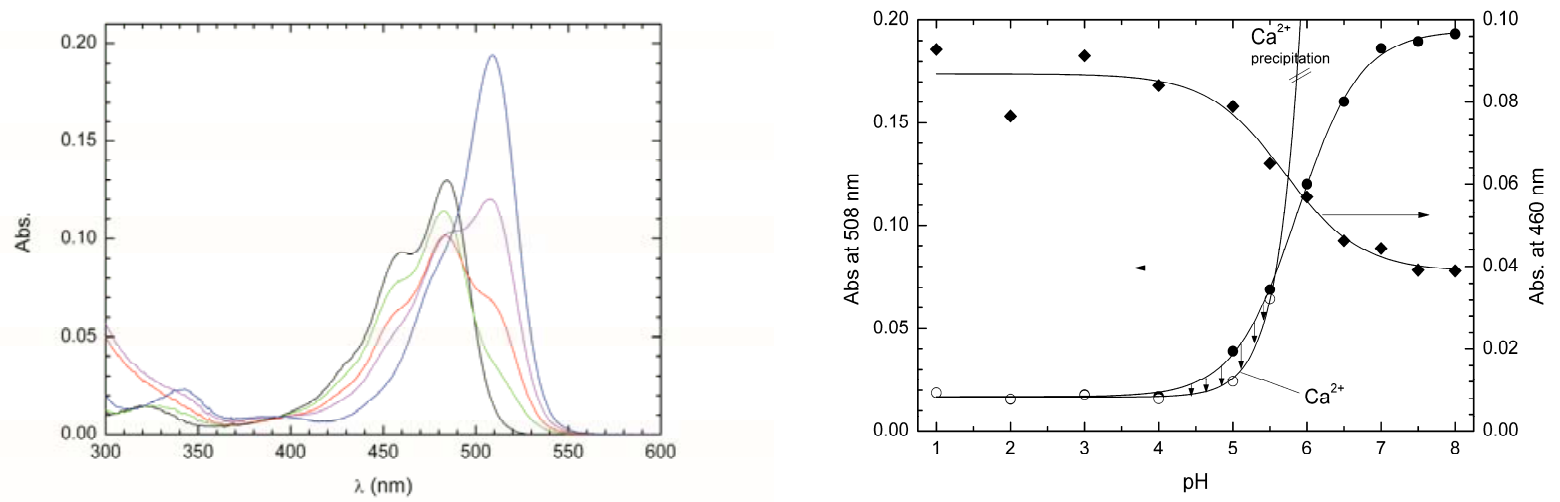

Figure 62. Left: absorption spectra of compound $\mathbf{I c}(\mathrm{C}=5.7 \mu \mathrm{M})$ at various $\mathrm{pH}$ values in aqueous buffer solutions: black line: $\mathrm{pH}=1$, green line: $\mathrm{pH}=5$, red line: $\mathrm{pH}=5.5$, purple line: $\mathrm{pH}=6$, blue line: $\mathrm{pH}=8$. Right: absorption of aqueous solutions of compound Ic at 508 $\mathrm{nm}$ (filled circles, left axis) and at $460 \mathrm{~nm}$ (filled rhombs, right axis) plotted as a function of the $\mathrm{pH}$; the absorption (at $508 \mathrm{~nm}$ ) of aqueous solutions of compound Ic with calcium additions (hollow circles, left axis) plotted as a function of $\mathrm{pH}$.

The absorption spectra of the aqueous solutions of compound Ic in the $\mathrm{pH}$ range from 1 to 5 shows two bands at 457 and at $483 \mathrm{~nm}$. The first one is assigned to the form Ic' (Scheme 29) with a protonated nitrogen atom. The second band is assigned to the form Ic" with a

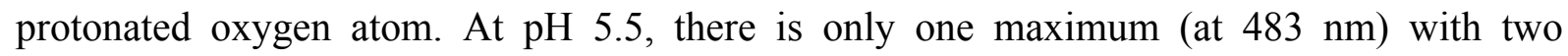
shoulders. The left shoulder, at $460 \mathrm{~nm}$, corresponds to the residual form $\mathbf{I c}^{\prime}$ (+ signal from the zwitter-ionic form $\mathbf{I c}^{\sim}$ ), and the second shoulder (at $509 \mathrm{~nm}$ ) corresponds to the neutral form $\mathbf{I c}^{\sim}$. A further increase in the $\mathrm{pH}$ (up to $\mathrm{pH}=6$ ) decreases the intensity of the shoulder at $460 \mathrm{~nm}$, so that the signal of the form $\mathbf{I c}^{\prime}$ disappeares, but the signal of the zwitter-ionic form $\mathbf{I c}^{\sim}$ still remains. 


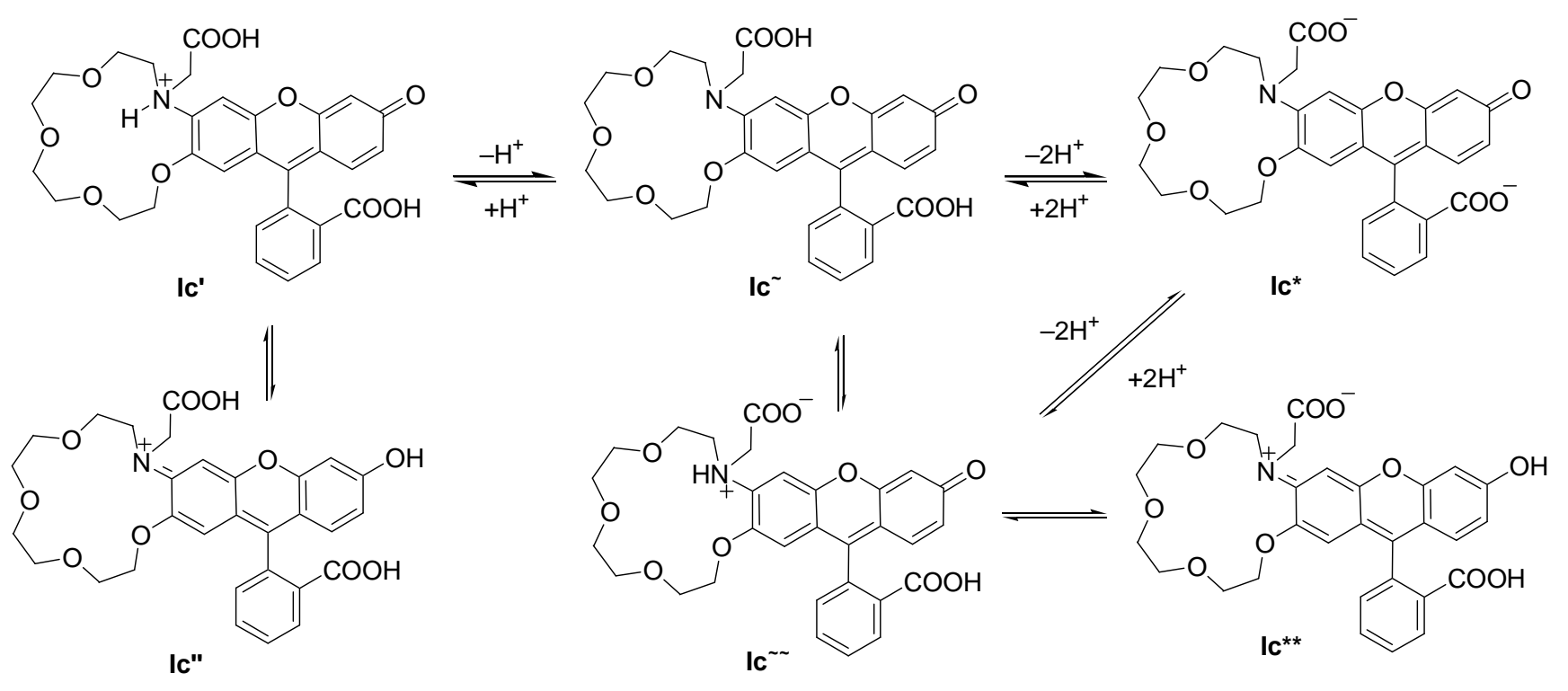

Scheme 29. Various protonated forms of the indicator Ic in aqueous solutions at different $\mathrm{pH}$ values.

At $\mathrm{pH}=6$, the intensity of the band at $509 \mathrm{~nm}$ increases, and the band at $485 \mathrm{~nm}$ transforms into a shoulder. In a basic medium $(\mathrm{pH}=8)$, the absorption maximum attributed to the form $\mathbf{I c}^{\sim}$ (at $460 \mathrm{~nm}$ ) disappeares, and the intensity of the absorption of the form $\mathbf{I c}^{\sim}$ increases, owing to an increase of the fraction of compound Ic in the form $\mathbf{I c}^{\sim}$ and a conversion also of compound Ic into the form $\mathbf{I c}^{*}$ (which has the same position of its absorption maximum at $509 \mathrm{~nm}$ ). In this instance, the form Ic $\mathbf{I c}^{\prime \prime}$ is absent, and it probably is replaced by the form $\mathbf{I c}^{* *}$ (which presumably has the same position of its absorption maximum at $483 \mathrm{~nm}$ ), because this zwitter-ionic form could be formed from Ic" by deprotonation of a carboxy group. At $\mathrm{pH}=8$, the form $\mathbf{I} \mathbf{c}^{* *}$ is represented in the absorption spectrum by a shoulder at $483 \mathrm{~nm}$.

As far as the independence of the spectroscopic signal from the $\mathrm{pH}$ value at $\mathrm{pH}=7-8$ is concerned, the wavelength of $508 \mathrm{~nm}$ seems to be preferable for practical applications, because there are smaller changes in absorption in this interval of $\mathrm{pH}$, compared to those at $460 \mathrm{~nm}$ (see right plot in Figure 62). 

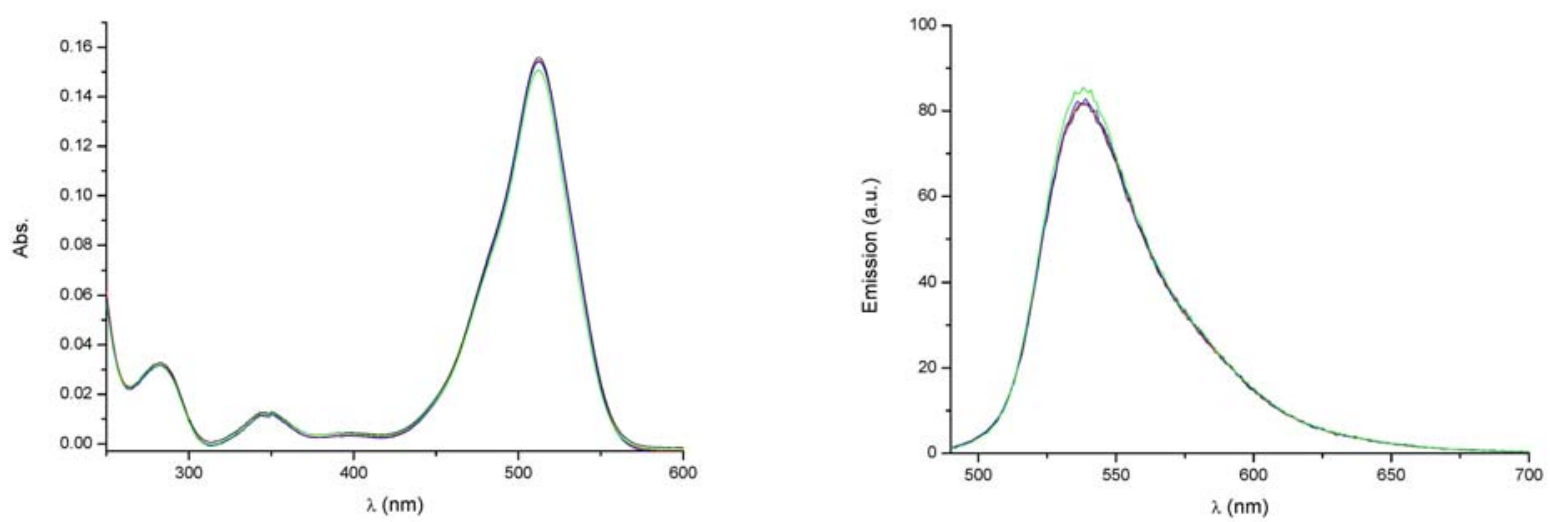

Figure 63. Small changes in the absorption (left) and fluorescence (right) spectra of compound Ic $(3.6 \mu \mathrm{M}$ in an aqueous buffer solution at $\mathrm{pH}=7.2)$ with free $\left[\mathrm{Ca}^{2+}\right]$ values ranging from 0 to $0.1 \mathrm{M}$; curves corresponding to various concentrations of $\mathrm{Ca}^{2+}$ are drawn in different colors: absorption and emission (black line - pure indicator, red line - pure indicator +10 equiv. of EDTA, blue line - pure indicator +10 equiv. of EDTA +10 equiv. of $\mathrm{Ca}^{2+}$, green line $-100 \mathrm{mM}$ of $\mathrm{Ca}^{2+}$ ).

Since in the preliminary measurements the sensitivity of compound Ic to calcium ion concentrations no significant changes (data not shown) were observed then we decided to use EDTA to remove the possible reason of the absence of spectroscopic signals. Up to 10 equivalents of EDTA were added to bind the possible cations, which could be located in the cavity surrounded by the crown-ether ring and the carboxy group. Therefore, in order to provide a starting point for the measurements, we bound the excessive EDTA by the addition of 10 equivalents of calcium chloride $\left(\mathrm{CaCl}_{2}\right)$. Then we performed the main measurements. Unfortunately, this indicator displayed only a very small decrease in its absorption and small increase in its fluorescence spectra with very high calcium ion concentrations (100 mM). Such response is too weak, to apply compound Ic as calcium indicator.

\section{Synthesis of $N, N, N^{\prime}, N^{\prime}$-tetramethylrhodamine (TMR) derivatives with an azacrown-ether ring fused with the 9-(2-carboxy)phenyl ring}

All other target structures IIa-b represent derivatives of TMR. For their preparation, we had to condense 3-(N,N-dimethylamino)phenol (71) with a phthalic anhydride bearing a fused azacrown system (compound 72a) or a phthalic anhydride with two different substituents in positions 4 and 5, from which the azacrown ether would later be constructed (e. g. compound 74 in Scheme 31). 


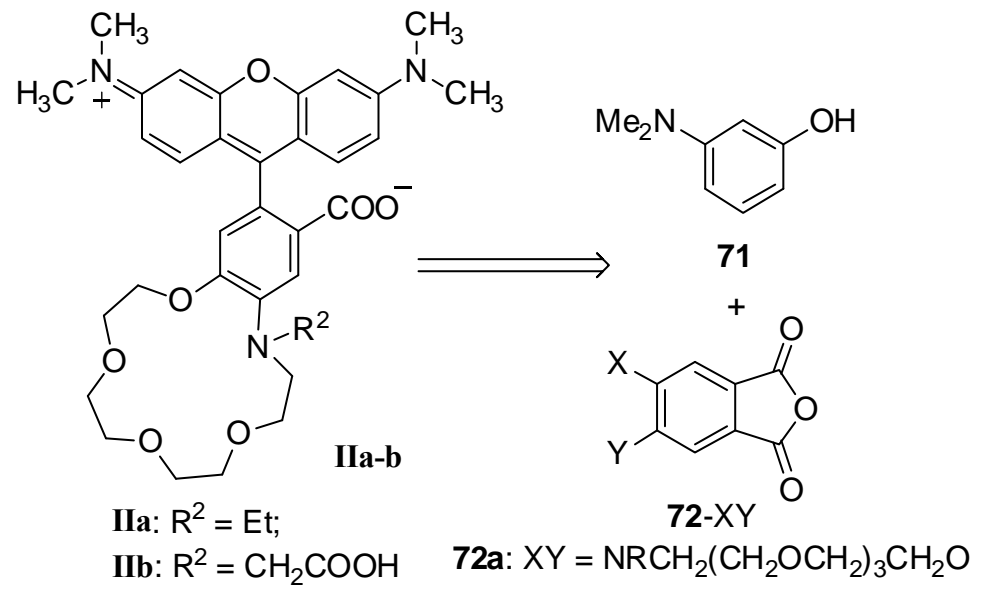

Scheme 30. Building blocks 71 and 72a from which the target fluorescent sensors IIa-b may be prepared.

The latter possibility appeared to be simpler and easier, and therefore, we embarked on the synthesis of the TMR derivate 73a (Scheme 31).

\subsection{Synthesis via the initial formation of TMR derivatives}

The TMR derivative with a nitro and a methoxy group in the phenyl ring (compound 73a in Scheme 31) was chosen as a precursor for the required substances IIa-b.

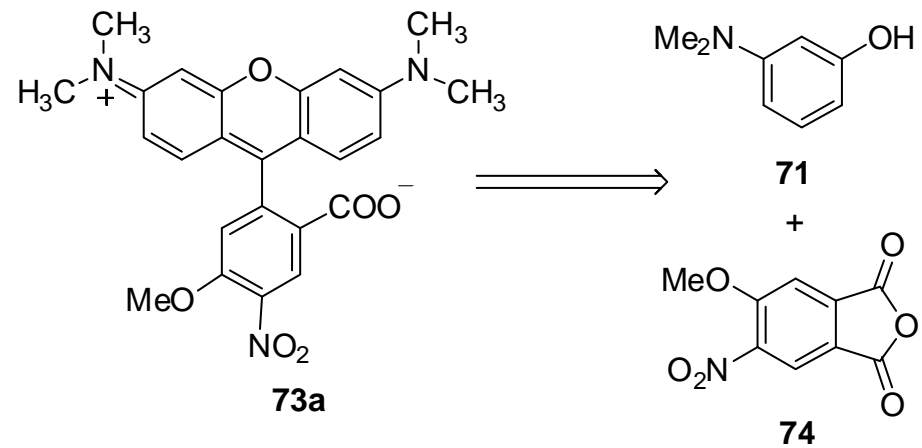

Scheme 31. Rhodamine 73a and building blocks (71 and 74).

The known synthesis of 4-methoxy-5-nitrophthalic anhydride (74) starts from 1,2dimethyl-4,5-dinitrobenzene (77). ${ }^{[106]}$ This symmetrical dinitro compound 77 was prepared in $50 \%$ overall yield by sequential oxidation of the commercially available 4,5-dimethyl-2nitroaniline (75) with $\mathrm{K}_{2} \mathrm{~S}_{2} \mathrm{O}_{8} / \mathrm{H}_{2} \mathrm{SO}_{4}$ (to the corresponding nitroso compound 76) and subsequently with fuming nitric acid. ${ }^{[107]}$ Conversion of the $o$-xylene derivative 77 into the corresponding phthalic acid $\mathbf{7 8}$ was achieved (though in low yield) by prolonged heating in a sealed tube with $25 \%$ aq. $\mathrm{HNO}_{3}$ at $180{ }^{\circ} \mathrm{C}$. 


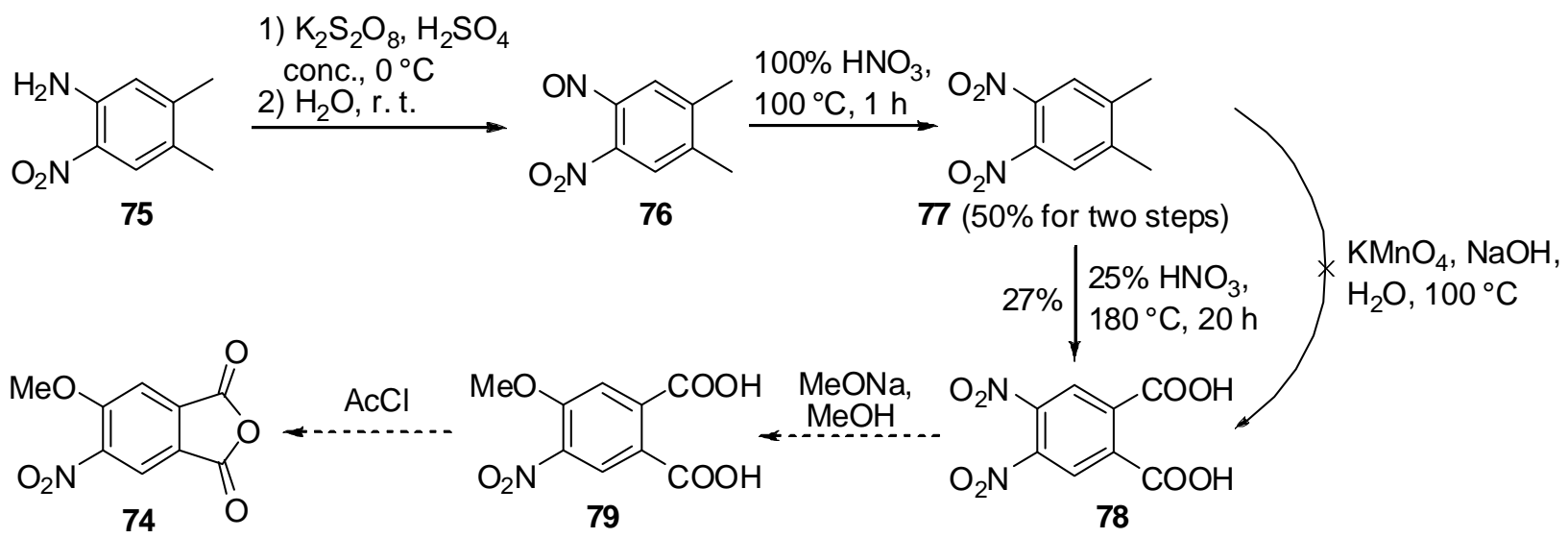

Scheme 32. Preparation of compound $\mathbf{7 8}$ as a precursor for 4-methoxy-5-nitrophthalic anhydride $74 .^{[106,107]}$

The authors of the original report ${ }^{[106]}$ managed to achieve higher yields (up to $63 \%$ ) by repeating the oxidation of the isolated unreacted compound 77 . The entire procedure could not be scaled-up, because of the high risk of an explosion. Therefore, we tried to find a more productive and safe route to compound $\mathbf{7 4}$.

Oxidation of compound 77 with basic aq. $\mathrm{KMnO}_{4}$ did not provide the dicarboxylic acid 78. ${ }^{[106]}$

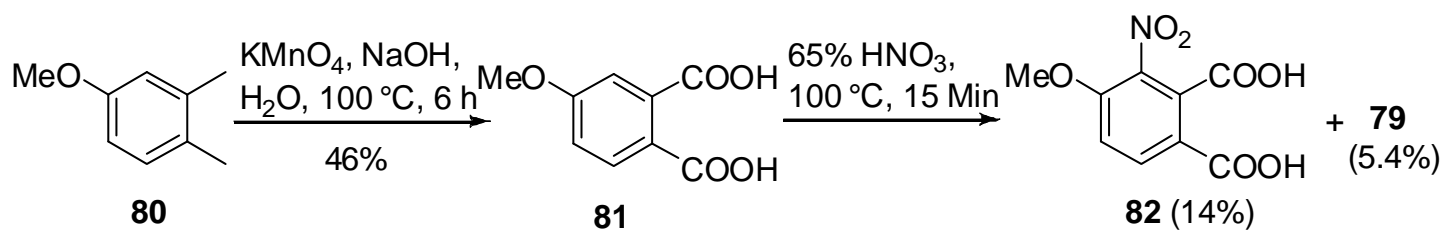

Scheme 33. An attempted alternative approach to 4-methoxy-5-nitrophthalic acid 79. ${ }^{[108,109]}$

A possible alternative approach to the dicarboxylic acid $\mathbf{7 9}^{[109]}$ is presented in Scheme 33. Nitration of 4-methoxyphthalic acid $(\mathbf{8 1})^{[108]}$ provided the required compound $\mathbf{7 9}$ in a very low yield of 5.4\%. Another regioisomer, isolated from the reaction mixture, turned out to be compound $\mathbf{8 2}$ (Scheme 33). It is interesting that the more sterically congested compound $\mathbf{8 2}$ was formed in higher yield (14\%) than the less sterically crowded substance.

One more idea, how to produce the acid 79, was based on the following observations. If it is not possible to oxidize compound $\mathbf{7 7}$ to the corresponding phthalic acid with $\mathrm{KMnO}_{4}$, but it is possible to oxidize of 3,4-dimethylphenyl methyl ether $\mathbf{8 0}$ to 4-methoxyphthalic acid $\mathbf{8 1}$, then it may be possible to oxidize 4,5-dimethyl-2-nitrophenyl methyl ether $\mathbf{8 3}$ to the acid $\mathbf{7 9}$. 


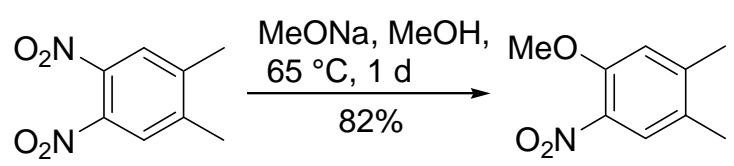

77

83

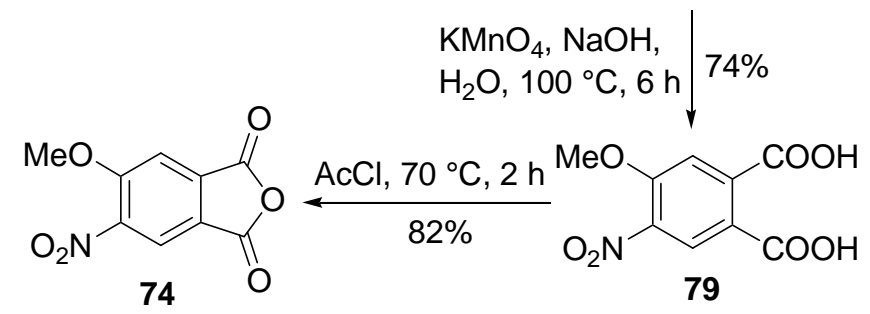

Scheme 34. An easy way to 4-methoxy-5-nitrophthalic anhydride 74 from 4,5-dinitro-1,2dimethylbenzene (77).

Eventually, we synthesized the phthalic anhydride $\mathbf{7 4}$ in a very efficient way (see Scheme 34). Aromatic nucleophilic substitution of the nitro group in compound 77 with $\mathrm{MeONa}$ afforded compound $\mathbf{8 3}$ in high yield. Oxidation of the methyl groups in $\mathbf{8 3}$ with basic aq. $\mathrm{KMnO}_{4}{ }^{[108]}$ led to the phthalic acid 79, which cyclized by heating in acetyl chloride ${ }^{[106]}$ and to produce the anhydride 74. It was necessary to optimize the conditions of the first step, in order to achieve the highest possible yield of compound $\mathbf{8 3}$ and to avoid the formation of side products (e. g. substituted diphenylmethanes arising from an $\mathrm{S}_{\mathrm{N}} \mathrm{Ar}$-reaction between the deprotonated methyl group which may react with the starting compound 77, substituting one of the nitro groups).

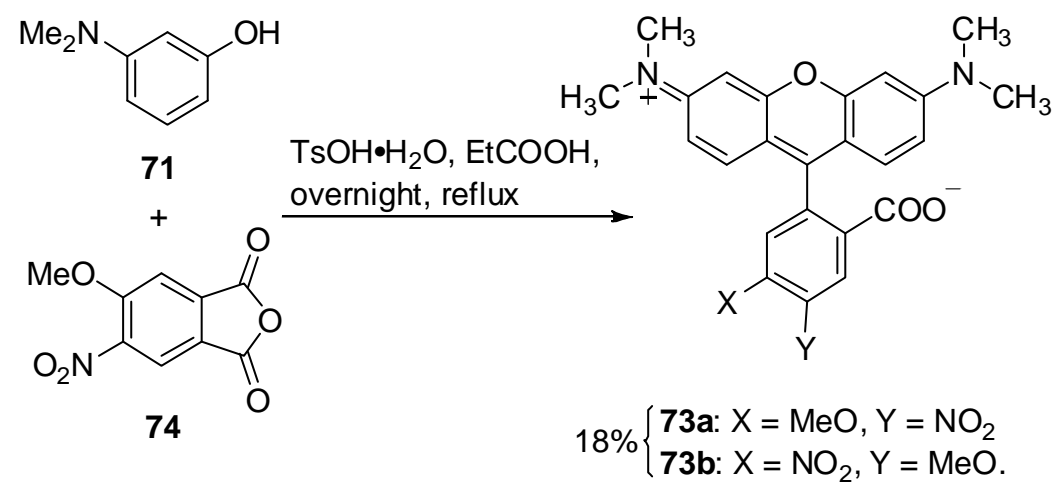

Scheme 35. Synthetic route to the TMR derivatives 73a-b.

The first approach to the TMR derivatives 73a,b included heating a mixture of the substituted phthalic anhydride $\mathbf{7 4}$ and aminophenol $\mathbf{7 1}$ in toluene. ${ }^{[110]}$ However, the yield of the target products 73a,b was very low (9\%). The second attempt was to use conditions as applied for the rhodol synthesis. The yield of the TMR derivatives 73a,b was increased to $18 \%$, but remained low. On for of that, the isomers $73 \mathbf{a}$ and $\mathbf{7 3 b}$ could not be separated. Therefore, this approach was not pursued any further. 


\subsection{Elaboration of a route to phthalic acid derivatives with an azacrown-ether fused to the aromatic ring}

Fusion of the azacrown ether with the positions 4 and 5 of the aromatic ring in phthalic anhydride (compounds $\mathbf{8 4 a , b}$ ) would provide an easy route to the required fluorescent sensors IIa,b (Scheme 36).<smiles>[R2]CN1CCOCCOCCOCCOc2cc(C(=O)[O-])c(-c3c4ccc(=[N+](C)C)cc-4oc4cc(N(C)C)ccc34)cc21</smiles>

IIa: $\mathrm{R}^{2}=\mathrm{Et}$;

IIb: $\mathrm{R}^{2}=\mathrm{CH}_{2} \mathrm{COOH}$<smiles>[R]N(CC)c1cc2c(cc1OCCOCC)C(=O)OC2=O</smiles>

84a: $R^{2}=E t ;$

84b: $\mathrm{R}^{2}=\mathrm{CH}_{2} \mathrm{COOH}$

Scheme 36. A retrosynthetic consideration of an alternative approach to compounds IIa-c based on the phthalic anhydrides $\mathbf{8 4 a}, \mathbf{b}$.

In order to fuse the azacrown-ether system with the aromatic ring in phthalic anhydride, it was necessary to use its protected form (or a synthetic equivalent), because the multistep procedures for the introduction and cyclization of an azacrown ring involve various highly reactive substances. Methyl groups appeared to be a bad choice, because they require oxidation in the final step, in the presence of the electron-donating azacrown-ether ring, which makes the aromatic nucleus very susceptible to oxidation. Therefore, we chose another synthetic equivalent of the phthalic anhydride, its $N$-methyl imide 90, which is depicted in Scheme 37.

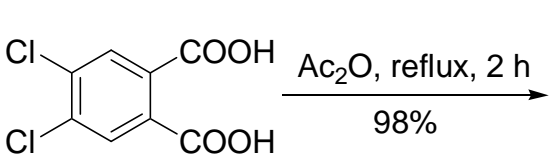

85

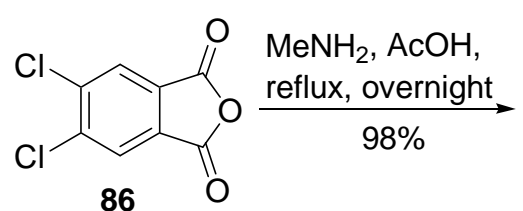

86<smiles>CN1C(=O)c2cc(Cl)c(Cl)cc2C1=O</smiles>

87

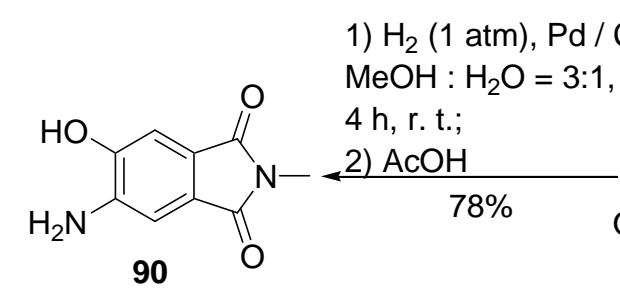
1) $\mathrm{H}_{2}$ (1 atm), Pd / C

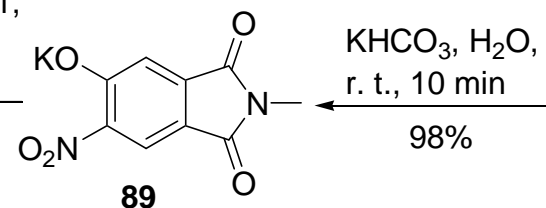

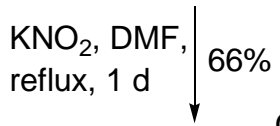<smiles>CN1C(=O)c2cc(O)c([N+](=O)[O-])cc2C1=O</smiles>

88

Scheme 37. Synthesis of the aminophenol 90 as a key intermediate. 
To access compound 90, commercially available 4,5-dichlorophthalic acid (85) was transformed into its anhydride $\mathbf{8 6},{ }^{[111]}$ and the latter then to the $N$-methyl imide $87 .{ }^{[12]} \mathrm{A}$ twofold nucleophilic substitution of the two chlorine atoms in $\mathbf{8 7}$ was performed with $\mathrm{KNO}_{2}$ in $\mathrm{DMF}$ at ca. $150{ }^{\circ} \mathrm{C}$ to give the ortho-hydroxynitro derivative $\mathbf{8 8}$. The formation of the latter may be explained, if we assume that one chlorine atom is replaced with a nitro group, and another one with an $\mathrm{O}=\mathrm{N}-\mathrm{O}^{-}$group $\left(\mathrm{KNO}_{2}\right.$ acts as an ambident nucleophile). The nitrite ester group is then hydrolyzed upon aqueous work-up. ${ }^{[11]}$ Catalytic reduction of the potassium salt $\mathbf{8 9}^{[113]}$ gave 4-amino-5-hydroxy- $N$-methylphthalimide (90).

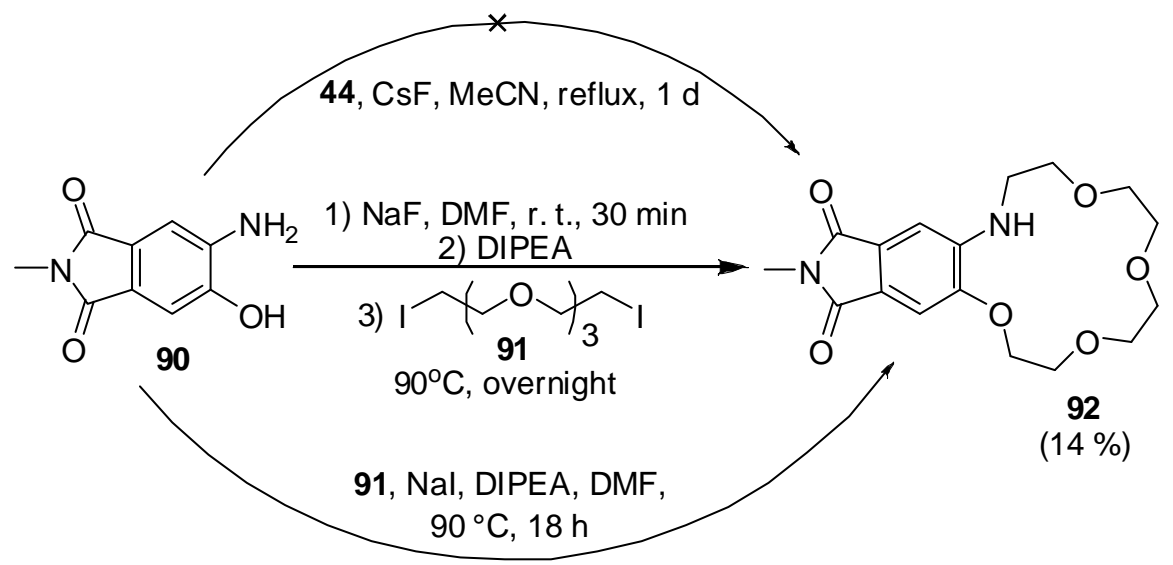

Scheme 38. Synthetic approaches to azacrown- $N$-methylphthalimide 92.

With the required building block 90 at hand, we studied the conditions, under which the azacrown ring can be build-up using the hydroxy and amino groups in the $o$-position to each other (Scheme 38). For that, it was necessary to use the diiodide 91 prepared from tetraethylene glycol dichloride $(\mathbf{5 0}),{ }^{[114 a]}$ because ditosylate $\mathbf{4 4}$ failed to provide the required macrocycle 92. Even with the diiodide 91 the highest achieved yield was only 14\%. Therefore, we turned to a step-wise construction of the azacrown ring using tetraethylene glycol derivatives 54-Ac and 54-Z ${ }^{[114 b]}$ (Scheme 39). 

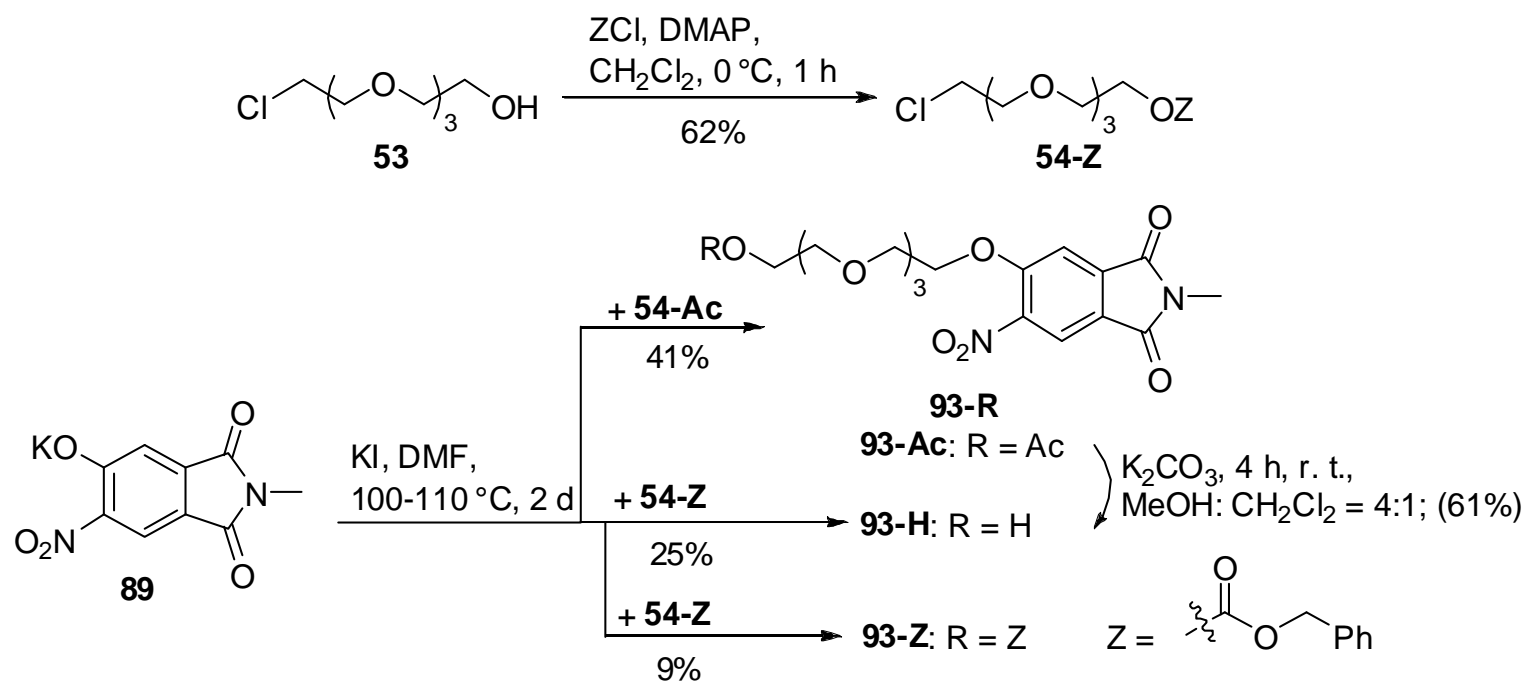

Scheme 39. Synthetic routes to alcohol 93-H.with the linear tetraethyleneglycol chain.

In a first attempt, the potassium salt 89 and the TEG-derivate 54-Ac were used as starting materials. Due to the presence of additional acceptor substituents in compound 89, the nucleophilic substitution proceeded more slowly than for compound $\mathbf{5 8}$ (Scheme 17). Even at $100-110{ }^{\circ} \mathrm{C}$, it took at least 2 days to achieve an acceptable conversion of compound 89 (in the presence of an equimolar amount of KI). In spite of that, the yield of compound 93-Ac was only moderate $(41 \%)$. Moreover, it was necessary to remove the acetyl protecting group in a separate step. Though the acetyl group could be removed under mild conditions $\left(\mathrm{K}_{2} \mathrm{CO}_{3}\right.$, $\mathrm{MeOH}$ ), the yield of the target alcohol 93-H (61\%) was far from being quantitative. Therefore, the overall yield of compound $93-\mathrm{H}$ in the two step sequence was only $25 \%$.

Employment of the alkylating agent of compound 54-Z instead of 54-Ac did not improve the yield of the intermediate 93-H (see Scheme 39).

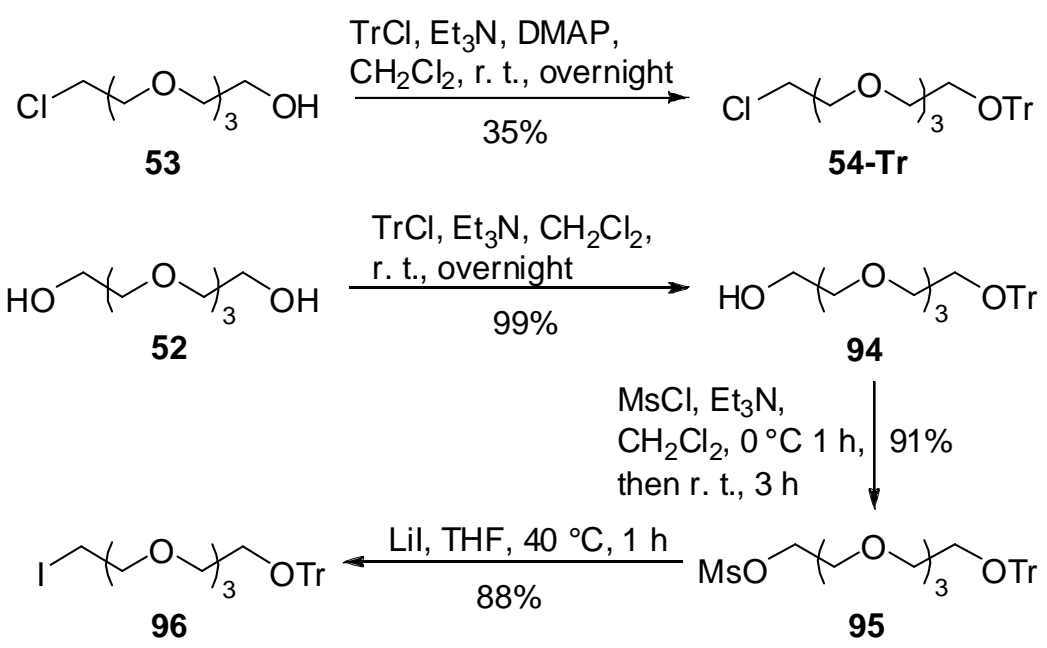

Scheme 40. Synthesis of alkylating reagents 54-Tr, 95, 96 
The trityl group was chosen as a new protection for the tetraethylene glycol derivatives (54-Tr, 95, 96) with good leaving groups $(\mathrm{Cl}$, Ms and I) (Scheme 40). The yield of the chloride 54-Tr was low (35\%). ${ }^{[15]}$ In the synthesis of compounds 95 and $\mathbf{9 6}$, the protecting group was introduced in the first step, by the reaction of compound $\mathbf{5 2}$ with chlorotriphenylmethane, which gave the ether 94 in high yield (99\%). ${ }^{[116]}$ The remaining free hydroxy group in the ether $\mathbf{9 4}$ was converted into mesylate to give compound $\mathbf{9 5}$ in $\mathbf{9 1 \%}$ yield ${ }^{[116]}$ from the latter, the iodide 96 was prepared in $88 \%$ yield. ${ }^{[117]}$

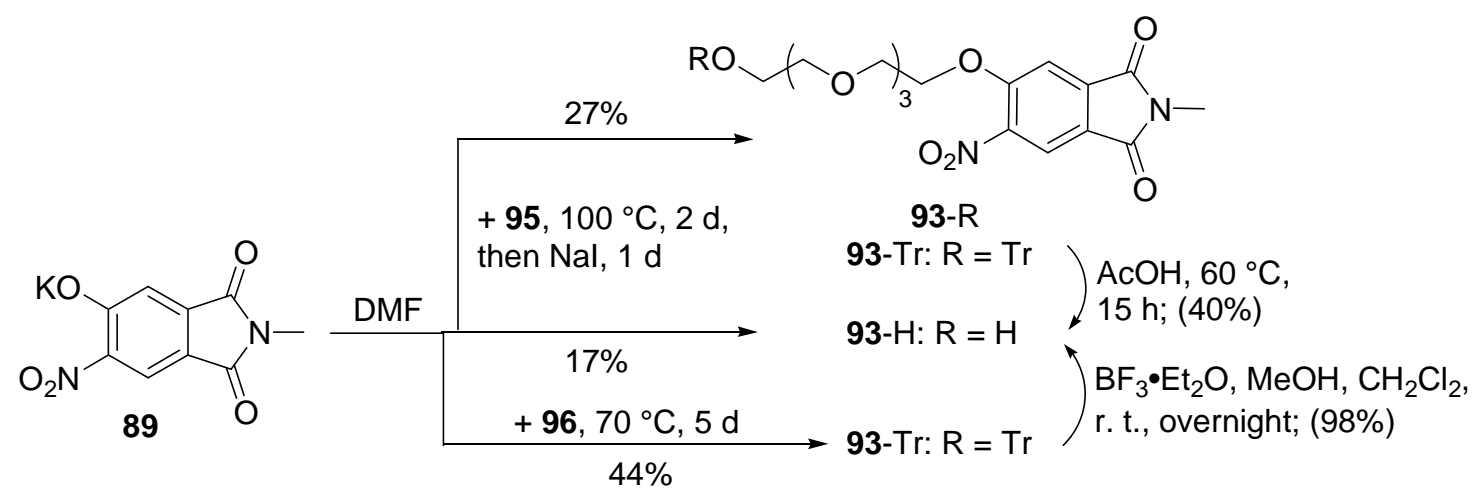

Scheme 41. Optimization of the conditions in the preparation of the alcohol $93-\mathrm{H}$.

In the first experiments, the mesylate 95 was used as an alkylating reagent. The overall yield of the alkylation products $(93-\mathrm{Tr}$ and $93-\mathrm{H})$ was higher than in the reaction of the salt 89 with the chloride 54-Z (44\% vs. 34\%). However, more by-products were detected, and a mixture of compounds was formed $(93-\operatorname{Tr}$ and $93-\mathrm{H})$. In order to increase the yield of the target product 93-Tr, another alkylating agent, the iodide 96, was used. To suppress the deprotection of the hydroxy group, the reaction temperature was decreased to $70{ }^{\circ} \mathrm{C}$, and a longer reaction time was applied (5 instead of 2 days). The highest conversion of the potassium salt 89 into the ether $93-\operatorname{Tr}$ was found to be $59 \%$; the content of the non-reacted starting material was $25 \%$. In order to prove our assumption that the decomposition of iodide 96 was quicker than its reaction with the potassium-salt 89, the mode of the iodide addition was changed. Half an equivalent of compound 96 was added in four portions (in the beginning and every $12 \mathrm{~h}$ ) to the reaction mixture. However, the conversion was found to be the same $(59 \%$ of $93-\operatorname{Tr}$ and $26 \%$ of 89$)$. Also, a change of the solvent to HMPTA in this reaction did not help to shift the equilibrium towards the target product 93-Tr. Unfortunately, the ratio became even worse, i. e. 49:37.

For the deprotection of the nitroether 93-Tr, two procedures were applied. Heating the starting material in glacial acetic acid ${ }^{[118]}$ afforded the target product $93-\mathrm{H}$ only in moderate yield (40\%). The main by-product in this reaction was the ester 93-Ac. Treatment of the ether 
93-Tr with a $2 \mathrm{M}$ methanolic solution of $\mathrm{BF}_{3} \cdot \mathrm{Et}_{2} \mathrm{O}^{[119]}$ gave the pure alcohol $93-\mathrm{H}$ in $98 \%$ yield.

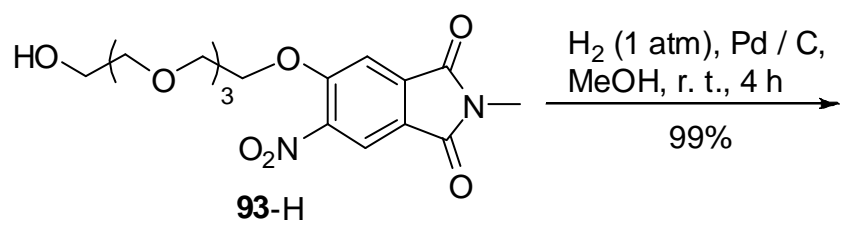<smiles>CN1C(=O)c2cc(N)c(OCCOCCO)cc2C1=O</smiles>

97

$\left.\begin{array}{l}\mathrm{TsCl}, \mathrm{Py} \\ 4{ }^{\circ} \mathrm{C}, 3 \mathrm{~d}\end{array}\right\rfloor 71 \%$

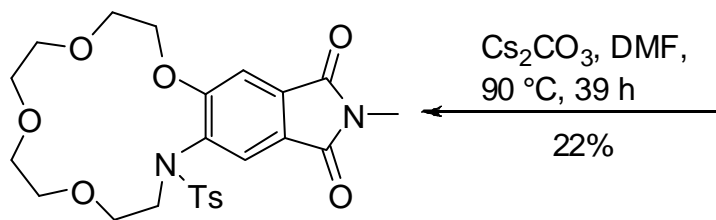

99

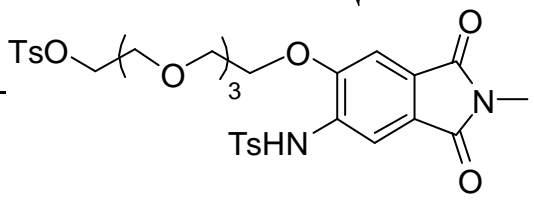

98

Scheme 42. Synthesis of the $N$-tosylated azacrown ether 99 by a ring-closing reaction sequence.

After that the nitro group in the alcohol 93-H was catalytically reduced, and compound $\mathbf{9 7}$ was converted into the bis-tosylate 98 (Scheme 42). Conventional conditions $\left(\mathrm{Cs}_{2} \mathrm{CO}_{3}, \mathrm{DMF}\right.$, $90{ }^{\circ} \mathrm{C}$ ) were used for the ring-closing reaction; unfortunately, the yield of the monotosylate 99 was low (even after the reaction time was increased from 24 to $39 \mathrm{~h}$ ).

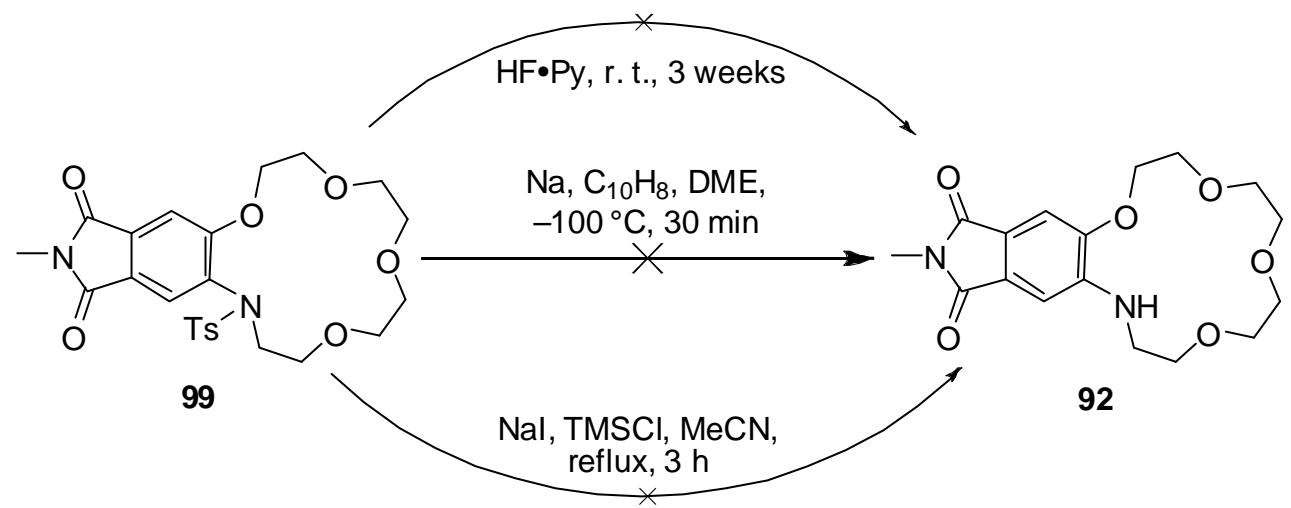

Scheme 43. Attempted $N$-detosylation of the crown ether 99.

Unfortunately, all approaches to recover the target crown ether 92 from its tosylamide 99 failed. Under mild detosylation conditions $\left(\mathrm{HF} \bullet \mathrm{Py}^{[120]}\right.$ or $\left.\mathrm{TMSI}^{[121]}\right)$, only the starting compound 99 was isolated; and with sodium naphthalenide, the reaction mixture did neither contain the starting material 99, nor the target crown ether 92. 


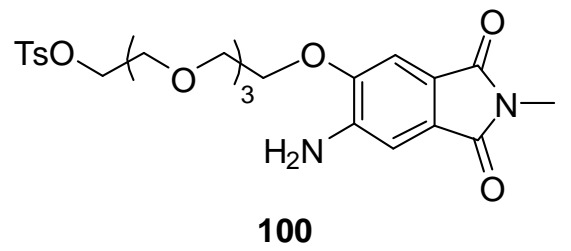

Figure 64. The intermediate buildings block 100.

In order to be able to efficiently detosylate of compound $\mathbf{9 9}$ we were forced to modify the synthesis and circumvent the detosylation step. Towards that, it was necessary to prepare the monotosylate 100. This compound could be produced not only by a selective tosylation of the amino alcohol 97, but also by tosylation of the nitro alcohol 93-H followed by reduction.

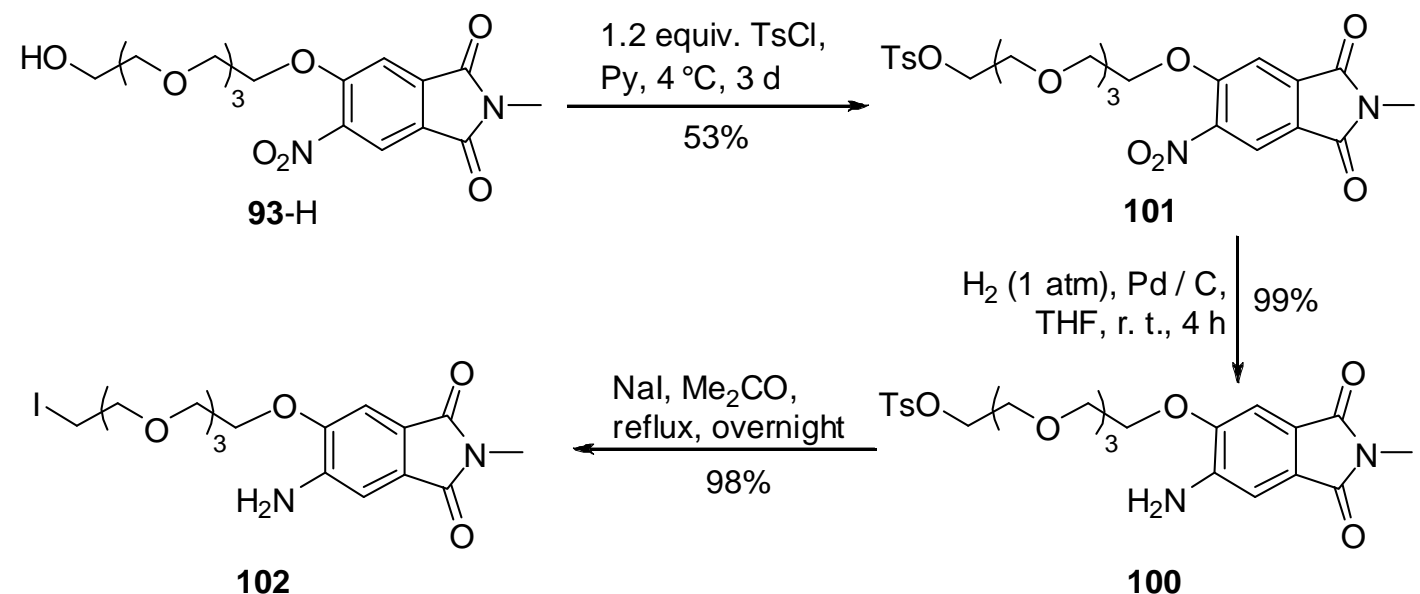

Scheme 44. Synthesis of the monotosylate 100 and the iodide 102.

Towards that, the nitrotosylate 101 was obtained from the nitroalcohol $93-\mathrm{H}$ in $53 \%$ yield according to the standard procedure. Then the nitro compound $\mathbf{1 0 1}$ was quantitatively converted into the amine $\mathbf{1 0 0}$ by catalytic hydrogenation. The iodide $\mathbf{1 0 2}$ was also produced by nucleophilic substitution of the tosyl group in compound $\mathbf{1 0 0}$ with iodide anion.

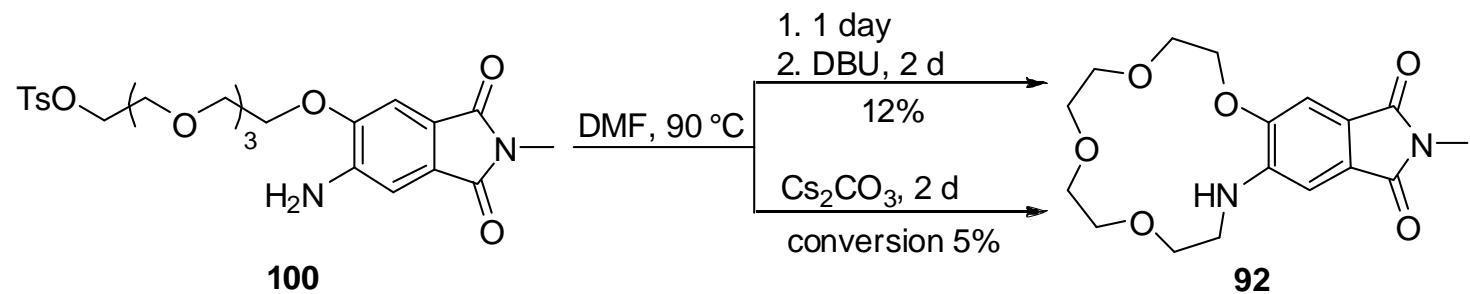

Scheme 45. Initial attempts to perform the cyclization to the crown ether $\mathbf{9 2 .}$

Initially, two bases (DBU and $\mathrm{Cs}_{2} \mathrm{CO}_{3}$ ) were tried in order to perform the cyclization to the crown ether 92 . In both cases, the target product 92 was observed, and in the case of DBU, 
this product was isolated. However, in these experiments the yield of the crown ether 92 was inacceptably low (maximum 12\%).

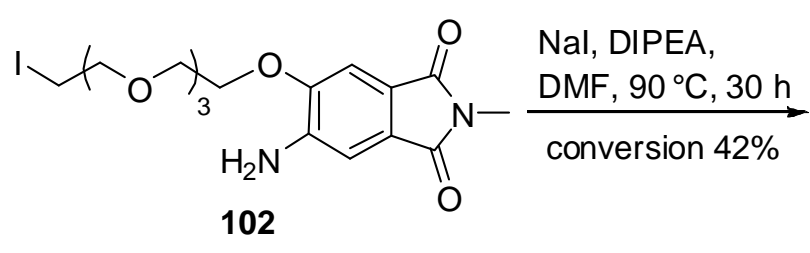

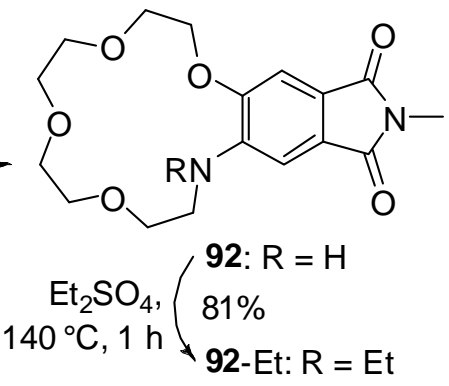

Scheme 46. Successful cyclization of the iodide 102 to the crown ether 92 and the synthesis of the ethylated derivative 92-Et.

To improve the yield of the target product 92, the tosyl leaving group was changed to an iodide (Scheme 46), in addition, sodium iodide was used as a templating agent, and a softer base (DIPEA) was applied. As a result, the yield of compound 92 increased to $42 \%$. Then the ethyl group was introduced into the crown ether 92 by heating it with diethyl sulfate at $140{ }^{\circ} \mathrm{C}$ for $1 \mathrm{~h} .^{[122]}$ 


\section{Chapter 3. Synthesis of SplAsH-based fluorescent labels for tetracysteine tags and their bioimaging tests}

\section{Development of biarsenical probes}

The first biarsenical probe - compound 103a - was described in 1998 by R. Tsien et al. ${ }^{[3]}$ It was based on fluorescein with two dithiarsolanic rings attached to positions 4 and 5 (see Table 5). Due to the reversibility of the reaction of As(III) with thiols, ethanedithiol ligands can be replaced with other thiols (e. g. cysteine residues in proteins).

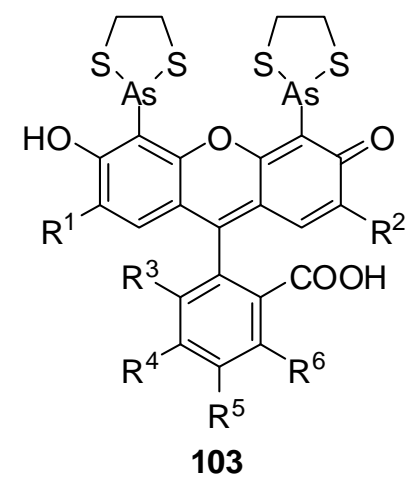

Table 5. FlAsH and its derivatives. ${ }^{[3,123-133]}$

\begin{tabular}{|c|c|c|c|c|c|c|c|}
\hline No. & Nickname & $\mathrm{R}^{1}$ & $\mathrm{R}^{2}$ & $\mathrm{R}^{3}$ & $\mathrm{R}^{4}$ & $\mathrm{R}^{5}$ & $\mathrm{R}^{6}$ \\
\hline $103 a$ & FlAsH & $\mathrm{H}$ & $\mathrm{H}$ & $\mathrm{H}$ & $\mathrm{H}$ & $\mathrm{H}$ & $\mathrm{H}$ \\
\hline $103 b$ & - & $\mathrm{H}$ & $\mathrm{H}$ & $\mathrm{H}$ & $\mathrm{H}$ & & $\mathrm{H}$ \\
\hline $103 c$ & - & $\mathrm{H}$ & $\mathrm{H}$ & $\mathrm{H}$ & $\mathrm{H}$ & & $\mathrm{H}$ \\
\hline $103 d$ & $\mathrm{Br}_{2} \mathrm{AsH}$ & $\mathrm{Br}$ & $\mathrm{Br}$ & $\mathrm{H}$ & $\mathrm{H}$ & $\mathrm{H}$ & $\mathrm{H}$ \\
\hline $103 e$ & - & $\mathrm{H}$ & $\mathrm{H}$ & $\mathrm{H}$ & $\mathrm{H}$ & & $\mathrm{H}$ \\
\hline
\end{tabular}




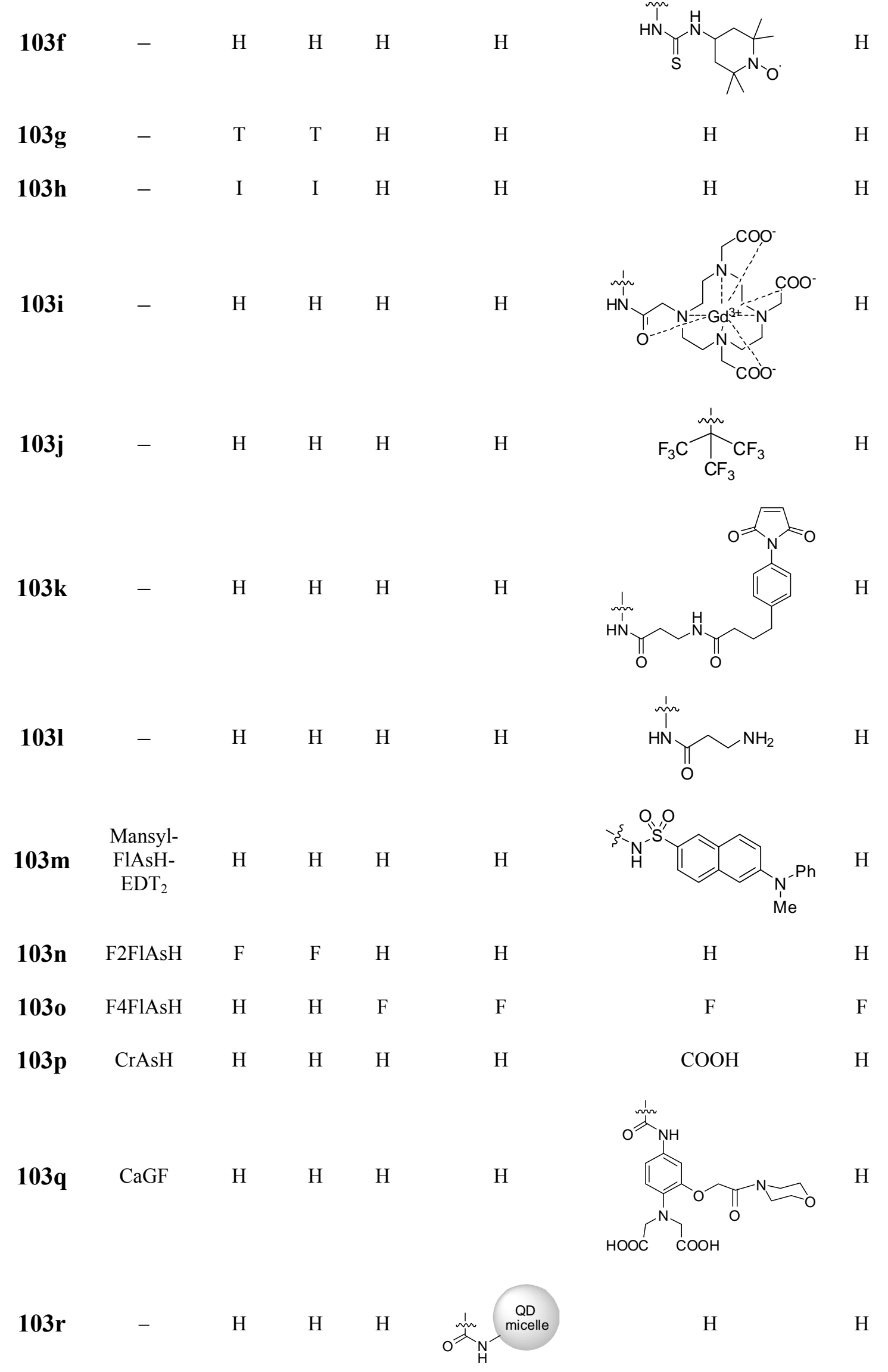




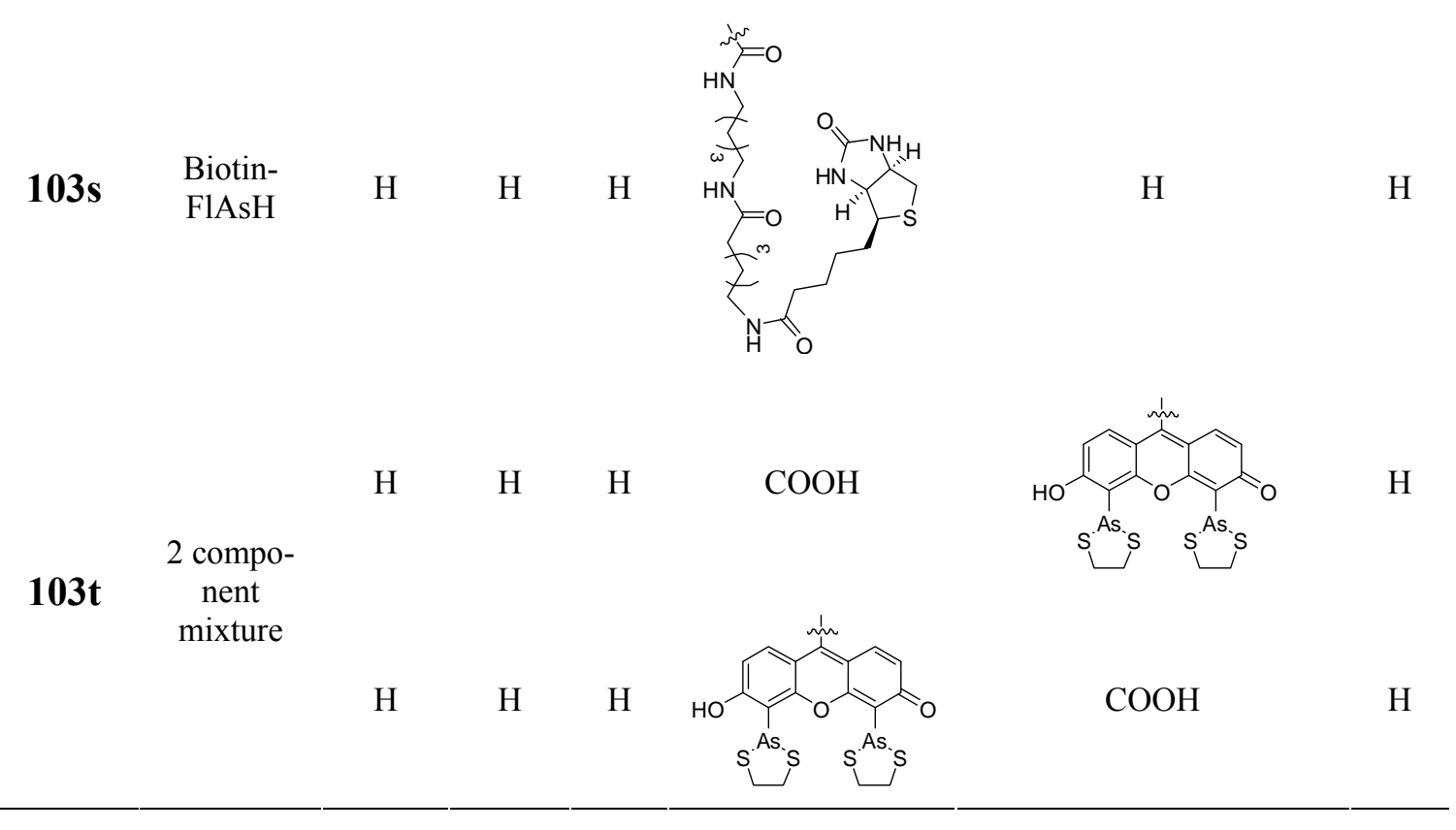

To provide a high selectivity of any new probe, a special peptide sequence with four cysteine residues was developed. ${ }^{[123]}$ This genetically encodable domain, the so-called "tetracysteine motif", may be represented as follows: CCXYCC, in which X, Y stands for any amino acid residue and $\mathrm{C}$ for cysteine. Owing to the required positions of the cysteine residues in the amino acid sequence, a highly stable complex $\left(K_{\mathrm{D}} \approx 10^{-11}\right)$ between two arsenic atoms and two pairs of cysteine residues is formed. The most stable complex was produced, with $\mathrm{X}=\mathrm{P}$ (proline) and $\mathrm{Y}=\mathrm{G}$ (glycine). Such kind of insertion (PG) between the two pairs of cysteines provides the required mutual orientation of the two pairs of thiol groups. Each pair of thiol group binds with one of the two arsenic atoms in compound 103a.

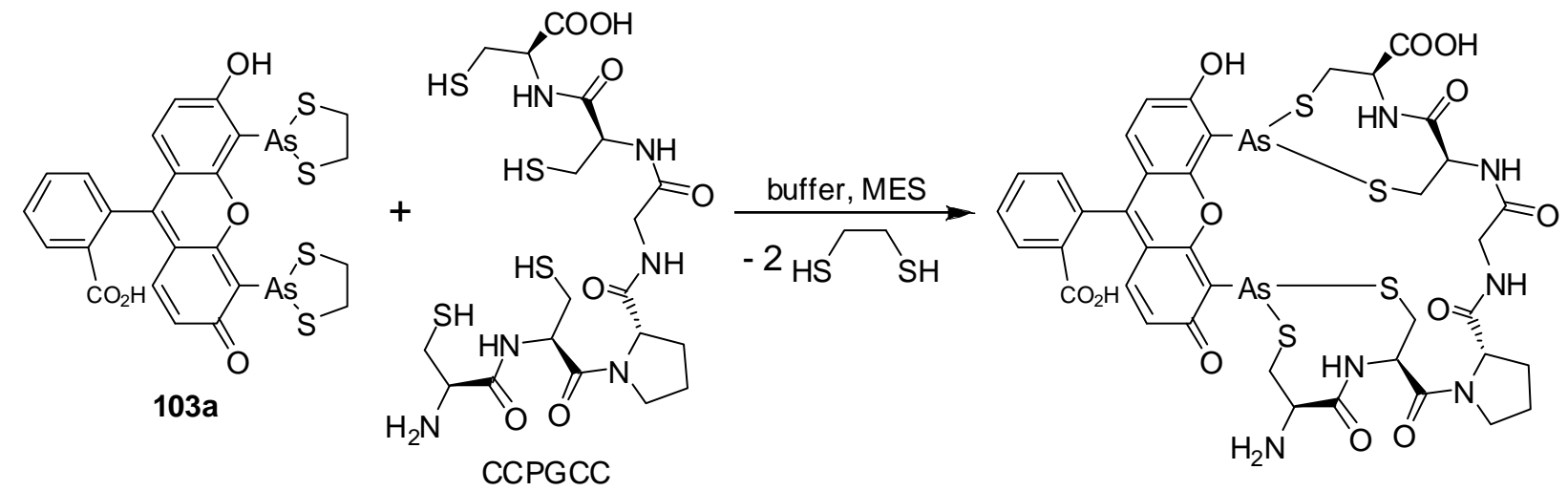

Scheme 47. Conjugation of FlAsH (compound 103a) with the CCPGCC peptide.

Besides its perfect binding ability, compound 103a undergoes an interesting transformation of properties. A solution of FIAsH in a buffer, which absorbs light in the region similar to fluorescein $\left(\lambda_{\max }(\mathbf{1 0 3 a})=508 \mathrm{~nm}\right)$, is almost non-fluorescent, due to the 
vibrational deactivation of the excited state of the F1AsH molecules. However, addition of the peptide or protein, with a CCPGCC amino acid sequence to this solution, makes it highly fluorescent, owing to the progress of the binding reaction (Scheme 47). Therefore, compound 103a can work not only as a selective binding unit, but also as a fluorescence label for any biomolecules with the "tetracysteine motif".

Owing to the intense fluorescence of the adducts (Scheme 47), and high affinity of compound 103a to tetracysteine-containing fragments, this substance and its analogues have found many applications in biological research, e. g., in the cell imaging, ${ }^{[3,123]}$ protein dynamics, ${ }^{[123 a]}$ protein purification, ${ }^{[124]}$ applications of the FRET method (fluorescence resonance energy transfer). ${ }^{[125]}$

FlAsH derivatives are used to deliver different labels to the target protein. Typical examples of such labels are: calcium chelators for the fixation of calcium nanodomains in cells (compounds 103e , $^{[123 b]} \mathbf{1 0 3} \mathbf{q}^{[126]}$ ), spin labels for studying of protein interactions by EPR (compound $\mathbf{1 0 3 f ^ { [ 1 2 3 b ] }}$ ), radioactive labels for the evaluation of protein traffic (compound

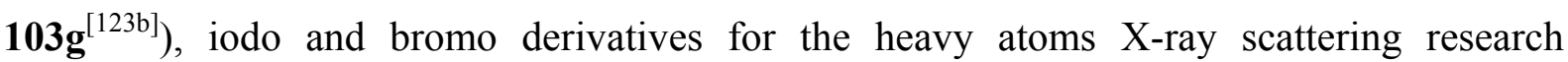
(compound $\mathbf{1 0 3 h}^{[123 \mathrm{~b}]}$ ), labels with paramagnetic ions for ${ }^{1} \mathrm{H}-\mathrm{NMR}$ spectroscopy (e. g. compound $\mathbf{1 0 3} \mathbf{i}^{[123 b]}$ ), ${ }^{19}$ F-NMR probes (compound $\mathbf{1 0 3} \mathbf{j}^{[123 b]}$ ), labels that consist of quantum dot micelles with persistent fluorescence (compound $103 \mathbf{r}^{[127]}$ ).

FlAsH derivatives may be used as bifunctional linkers. For example, it is possible to bind the target peptide or protein (decorated with the "tetracysteine motif") to: streptavidine (via biotinylated compound $\mathbf{1 0 3} \mathrm{b}^{[123 \mathrm{~b}]}$ ) or its derivatives (with the help of compound $\mathbf{1 0 3} \mathbf{s}^{[128]}$ ), the enzymes via a lysine residue (using compound $103 \mathrm{c}^{[123 \mathrm{~b}]}$ ), monothiol-modified nucleic acids

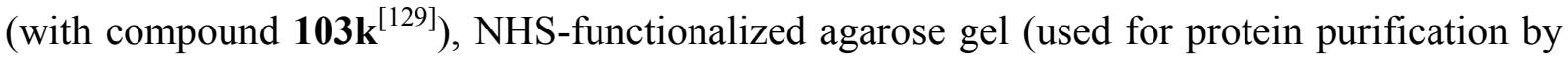
means of affinity chromatography [with the help of compound $\left.\mathbf{1 0 3 I}^{[124]}\right]$ ). The FlAsH dimer with two xanthene nuclei and four arsenics (compound $\mathbf{1 0 3 t}^{[123]}$ ) can induce the homodimerisation of peptides and proteins, which contain two tetracysteine tags.

Some of the FlAsH labels (compound $\mathbf{1 0 3 d}^{[123]}$ ) may be applied for staining objects, which may be indirectly visualized, i. e. by electron microscopy using the photooxidation of diaminobenzidine followed by staining with osmium salts. The dye 103d converts oxygen from the triplet into the singlet state, and the highly reactive singlet oxygen is capable of polymerizing diaminobenzidine into a densely localized precipitate. The latter could be stained with osmium tetroxide which can be detected by electron microscopy.

The compound Mansyl-FlAsH (103m) was produced in $2004 .^{[130]}$ It was found to be an indicator of protein conformations. This substance was attached to calmoduline protein 
decorated with a tetracysteine tag. Changes in fluorescence of the Mansyl-FlAsH label were observed upon conformational changes of calmoduline, the behaviour of which could be regulated by the calcium cation fluxes.

It should be noted, that most applications of FlAsH derivatives utilize only the selective binding of this probe to the tetracysteine motif, but not their fluorescent properties. FlAsH derivatives as fluorescent labels have some disadvantages: 1) a limited photostability and $\mathrm{pH}$ sensitivity of fluorescein in the physiological $\mathrm{pH}$ range ${ }^{[131]} 2$ ) a high fluorescence background (at ca. $500 \mathrm{~nm}$ ) in the cell staining experiments. ${ }^{[132]}$ The first drawback was overcome by the introduction of the fluorine atoms into the FlAsH structure (compounds 103n and 1030). ${ }^{[131]}$ Compound 103n was 50 times more photostable than the parent biarsenical probe (103a). Both fluorinated derivatives absorb light of ca. $500 \mathrm{~nm}$ two times stronger than compound 103a. Their fluorescence quantum yields are two times higher, and their $\mathrm{pH}$ sensitivity is lower. The introduction of fluorine atoms into the xanthene system of FIAsH resulted in a blue shift of the absorption and fluorescence maxima for complexes of the biarsenical probe with the dodecapeptide with 4 cysteine residues $\left(\lambda_{a b s}^{\max }=511 \mathrm{~nm}, \lambda_{f l}^{\max }=527 \mathrm{~nm}\right.$ for FlAsHdodecapeptide; $\lambda_{a b s}^{\max }=500 \mathrm{~nm}, \quad \lambda_{f l}^{\max }=522 \mathrm{~nm}$ for F2FlAsH-dodecapeptide). However, introduction of fluorine atoms into the benzene ring attached to the xanthene system produced the opposite effect ( $\lambda_{a b s}^{\max }=528 \mathrm{~nm}, \lambda_{f l}^{\max }=544 \mathrm{~nm}$ for F4FlAsH-dodecapeptide).

A new improved biarsenical probe was obtained by the introduction of an additional carboxy group into the benzene ring of FIAsH. ${ }^{[133]}$ This probe is called CrAsH (compound 103p). Owing to the higher hydrophilicity of the new probe, the background fluorescence signal (the result of the non-specific binding of FIAsH with proteins) was reduced 8-9 times. The relative enhancement of the fluorescence upon complex formation was the same as in the case of FlAsH (35 times). The absorption and fluorescence maxima of compound 103p were only slightly red-shifted in comparison with the FlAsH-peptide complex $\left(\lambda_{a b s}^{\max }=513 \mathrm{~nm}\right.$, $\lambda_{f l}^{\max }=534 \mathrm{~nm}$ vs. $\left.\lambda_{a b s}^{\max }=511 \mathrm{~nm}, \lambda_{f l}^{\max }=527 \mathrm{~nm}\right)$. As well as for compounds 103n and 1030, the lower $\mathrm{pH}$ sensitivity of compound 103p was observed (in the physiological $\mathrm{pH}$ range).

The "green" emission is not optimal for the use of FIAsH as a fluorescent label in biological research; typical limitations associated with this spectral region are: cellular absorbance of light, light scattering and cellular autofluorescence. The introduction of four electron-withdrawing groups into the benzene ring of FlAsH (in compound 1030) shifted the absorption and fluorescence maxima to the red only slightly. Therefore, another approach, i. e. the radical change of the fluorophore, was pursued. The phenoxazine compound $\mathbf{1 0 4}$ 
(without substituents) was chosen as a fluorescent core for the red-emitting biarsenical probe. Besides the required absorption and emission bands, the phenoxazine system provides a suitable position for the attachment of the dithiarsolanic rings.

Table 6. Phenoxazine-based biarsenical probes. ${ }^{[123 a, 134-136]}$

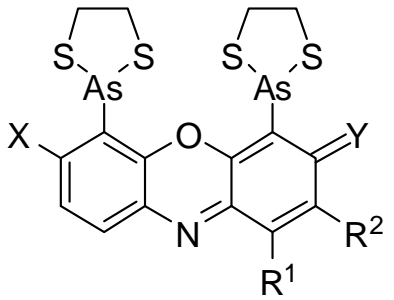

$104 a-b$

\begin{tabular}{cccccc}
\hline No. & Nickname & $\mathrm{R}^{1}$ & $\mathrm{R}^{2}$ & $\mathrm{X}$ & $\mathrm{Y}$ \\
\hline 104a & BArNile & & & & \\
104b & ReAsH & $\mathrm{H}$ & $\mathrm{H}$ & $\mathrm{O}$ & $\mathrm{O}$ \\
\hline
\end{tabular}

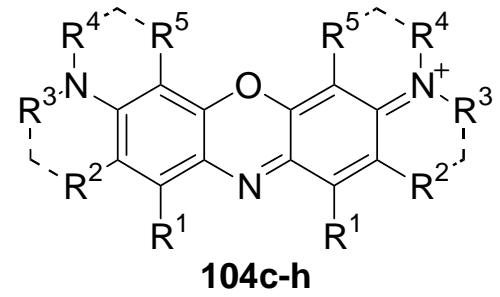

\begin{tabular}{|c|c|c|c|c|c|}
\hline No. & $\mathrm{R}^{1}$ & $\mathrm{R}^{2}$ & $\mathrm{R}^{3}$ & $\mathrm{R}^{4}$ & $\mathrm{R}^{5}$ \\
\hline $104 c$ & & $\mathrm{H}$ & $\mathrm{Me}$ & $\mathrm{Me}$ & $\mathrm{H}$ \\
\hline $104 d$ & $\begin{array}{l}1 \\
1 \\
\text { Sb } \\
\text { Sb }\end{array}$ & & & & \\
\hline $104 e$ & $\mathrm{H}$ & & & $\mathrm{H}$ & \\
\hline $104 f$ & $\mathrm{H}$ & & & $\mathrm{H}$ & $S_{b}^{b}$ \\
\hline
\end{tabular}




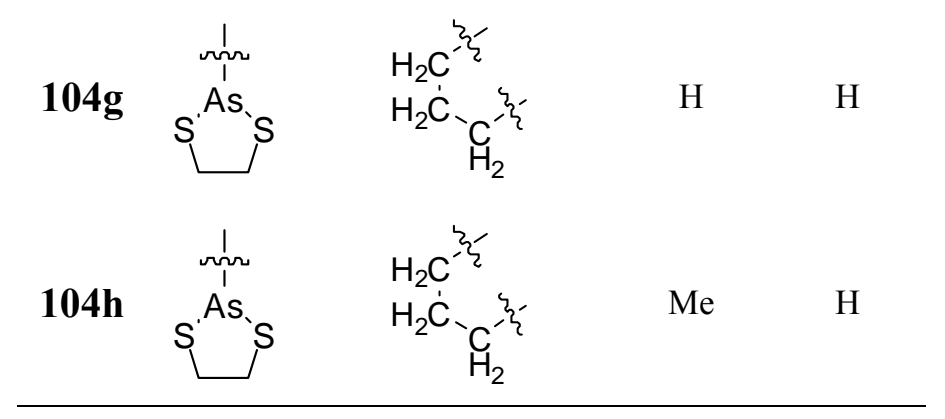

Compound 104a was the first example in this series. It represents an environmentallysensitive fluorescent probe for imaging conformational changes in proteins of living cells. ${ }^{[134]}$ The reported changes of the fluorescence signal of this probe are caused by conformational changes of the recombinant calmodulin, which are regulated by the calcium concentration in the medium. The fluorescence maximum of compound 104a is found in the region from 560 to $610 \mathrm{~nm}$ (depending on the solvent polarity).

The simplier phenoxazine derivative ReAsH was introduced by Adams et al. ${ }^{[123 a]}$ The absorption and fluorescence maxima of this biarsenical probe were significantly red-shifted in comparison with the FlAsH derivatives $\left(\lambda_{a b s}^{\max }=593 \mathrm{~nm}, \lambda_{f l}^{\max }=608 \mathrm{~nm}\right.$ ). Surprisingly, compound 104b which does not include heavy atoms, has shown a good photoconversion of triplet oxygen into singlet oxygen (revealed in the course of staining of the cellular objects with a polymer of diaminobenzidine and then with osmium in the process mentioned above). Compound 104b was used as a selective fluorescent label for the differentiation of "younger" and "older" proteins in the study of connexin trafficking. ${ }^{[135]}$

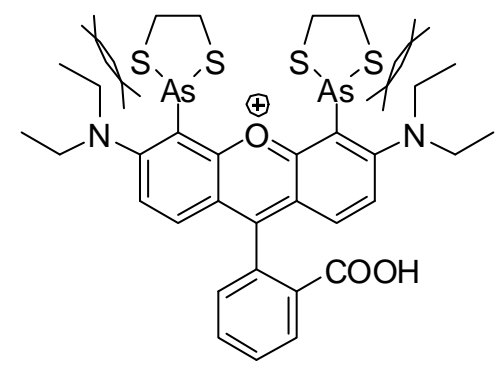

Figure 65. Biarsenical probe, based on Rhodamine B.

In order to shift the spectroscopic bands of phenoxazine biarsenical probes further into the red region, dialkylamino groups were introduced into the positions $\mathrm{X}$ and $\mathrm{Y}$ of compound 104a (see Table 6).

Interestingly, in the case of a biarsenical probe based on Rhodamine B, no changes in the fluorescence upon the formation of the complex of the model tetracysteine-containing peptide 
with the rhodamine-based biarsenical probe was reported. ${ }^{[123 a]}$ The main reason for that was the absence of quenching of the fluorescence in the initial state of the biarsenical probe (before the formation of the complex with the tetracysteine tag). The steric repulsions between the dithiarsolanic rings and the dialkylamino groups (see Figure 65) are responsible for that. In order to exclude these unfavorable interactions in the near IR (infrared) phenoxazine biarsenical probes of type 104, the alkyl substituents were fixed in rigid tetrahydroquinoline cycles (e. g. in compounds 104e, 104h). ${ }^{[136]}$ Another way to exclude such steric repulsions is to change the location of the dithiarsolanic rings. New locations were reported in the same paper (compounds 104c, 104g). In addition to the biarsenic probes, antimony analogues were also described. They possess a lower toxicity for cells (compounds 104d, 104f).

The use of phenoxazine-based biarsenical probes removes some spectroscopic limitations of the structure of a probe. However, it is still not possible to use some important dyes with (near) IR emission and some bright and photostable dyes with visible emission. A universal approach towards the solution of this problem was reported by Bhunia et al. ${ }^{[7]}$ It was suggested to separate the binding moiety and the fluorophore. This approach reminds one of the application of FIAsH for the binding of different labels to the target protein decorated with a tetracysteine tag. However, if we use FlAsH for this purpose, its emission spectrum may overlap with that of another fluorescent dye and an undesirable FRET effect may occur. Therefore, the non-emitting fluorescein spirolactam was used as a new binding unit in compound 105a. Compound 105a ( $\mathrm{SplAsH}$ ) has the same positions of the arsenic atoms as FIAsH. The same substitution pattern allows using the same peptide tags with four cysteine residues. 


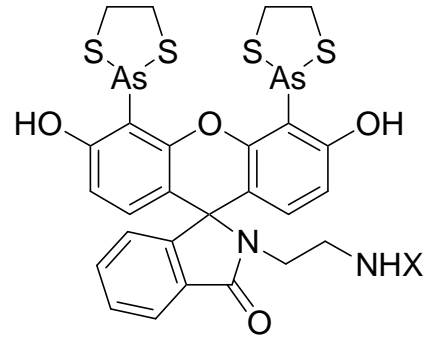

105a: SplAsH: $X=H$;

105b: SplAsH-Mant, $X=\mathrm{R}^{1}$;

105c: SplAsH-Dansyl, $X=R^{2}$;

105d: SpIAsH-DEAC, $X=\mathrm{R}^{3}$;

105e: SplAsH-ROX, $X=R^{4}$;

105f: SplAsH-Alexa594, $X=R^{5}$;

105g: SplAsH-NVOC-AB1, $X=R^{6}$<smiles>[R]CC(C)(C)S(=O)(=O)c1cccc2c(N(C)C)cccc12</smiles>

$R^{6}=$<smiles>CCN1CCCc2cc3c(cc21)Oc1cc2c(cc1N3CCCC(=O)C(C)(C)C)CCCN2CCCC(C)(C)C</smiles>

$\mathrm{R}^{3}=$<smiles>CCN(CC)c1ccc2cc(C(=O)O)c(=O)oc2c1</smiles>

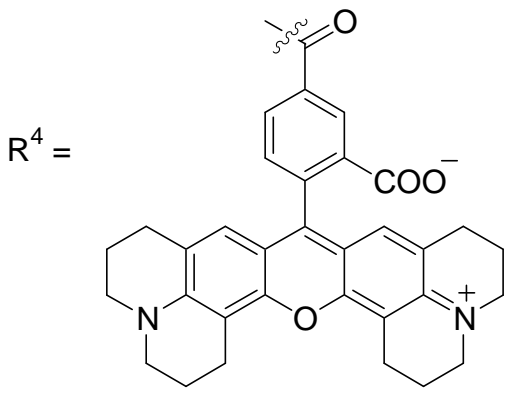

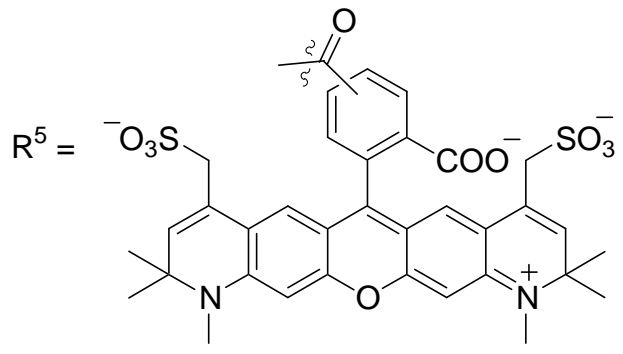

Figure 66. Biarsenical probes with non-fluorescent binding units called SplAsH. ${ }^{[7,137]}$

Compound 105a contains a free amino group, which could be covalently bound with any further labels. Some examples of these adducts were reported, ${ }^{[7]}$ the applied fluorophores were: $N$-methylantranylate (compound 105b), dansyl (compound 105c), diethylamino coumarine (compound 105d), carboxyrhodamine 101 (compound 105e), Alexa 594 (compound 105f). A SplAsH derivative with a photoactivable phenoxazine dye was also prepared (compound 105g). ${ }^{[137]}$ This probe starts to be highly fluorescent after cleavage of the NVOC-group followed by air oxidation.

\section{Synthesis of precursors for SplAsH-based fluorescent labels}

For the labelling of proteins (containing the tetracysteine tags) with switchable fluorescent dyes, we decided to use biarsenical probes consisting of the SplAsH unit attached to the rhodamine spiroamide residue. The difference between our biarsenical probes and the previously reported ones ${ }^{[7,137]}$ was the positions of the spectral maxima, and another photoactivation mode (for compound SplAsH-RhS-CF), i. e. the photoisomerization of the spirolactam instead of photocleavage, as in the case of compound $\mathbf{1 0 5 g}$. 
<smiles>[X]C12SCCSc3ccc(O)c1c3Oc1c2ccc(O)c1[As]1SCCS1</smiles>

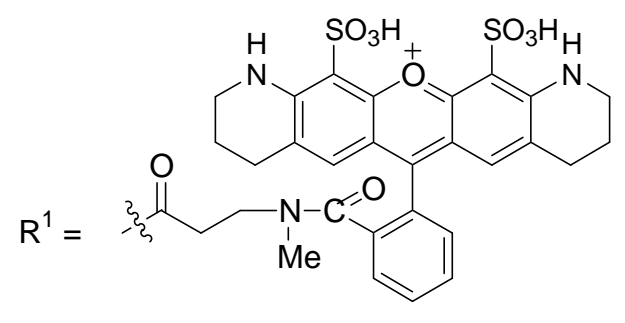

SpIAsH-RhS-OF: $\mathrm{X}=\mathrm{N}-\mathrm{CH}_{2}-\mathrm{CH}_{2}-\mathrm{NHR}^{1}$;

SplAsH-RhS-CF: $\mathrm{X}=\mathrm{N}-\mathrm{CH}_{2}-\mathrm{CH}_{2}-\mathrm{NHR}^{2}$;

SpIAsH-TAMRA: $X=\mathrm{N}-\mathrm{CH}_{2}-\mathrm{CH}_{2}-\mathrm{NHR}^{3}$.

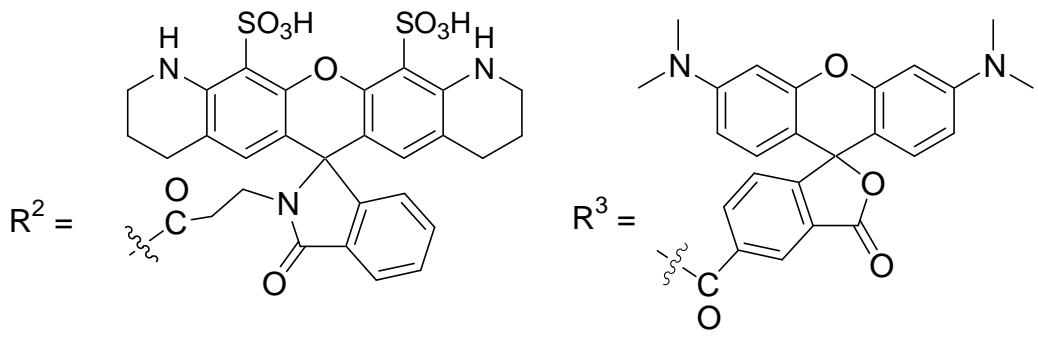

Figure 67. New biarsenical fluorescent and photoswitchable probes.

The spectral maxima of the new probes $\left(\lambda_{a b s}^{\max } / \lambda_{f l}^{\max }, \mathrm{nm}\right)$ are $540 / 560$ for compounds SplAsH-RhS-OF and SplAsH-RhS-CF (after photoactivation), 542/568 for compound SplAsH-TAMRA. The high hydrophilicity of the fluorescent moiety in the biarsenical probes SplAsH-RhS-OF and SplAsH-RhS-CF is an advantage in the conjugation reaction with proteins and peptides, because this process usually takes place in aqueous buffers.

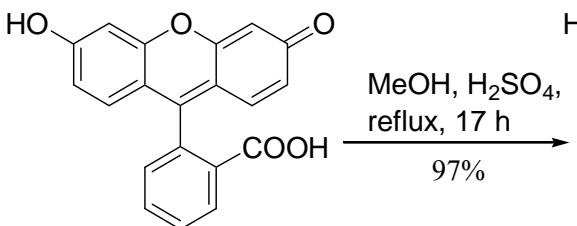

106<smiles>COC(=O)c1ccccc1-c1c2ccc(=O)cc-2oc2cc(O)ccc12</smiles>

107

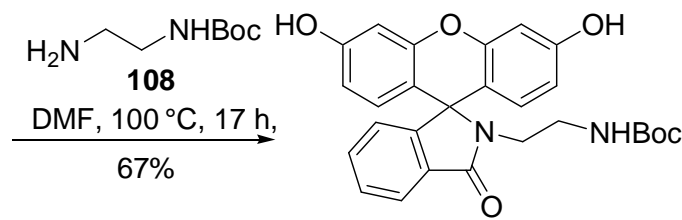

109

$\mathrm{Hg}(\mathrm{OAc})_{2}, \mathrm{EtOH}$, $2 \% \mathrm{AcOH}, 65^{\circ} \mathrm{C}, 90 \%$

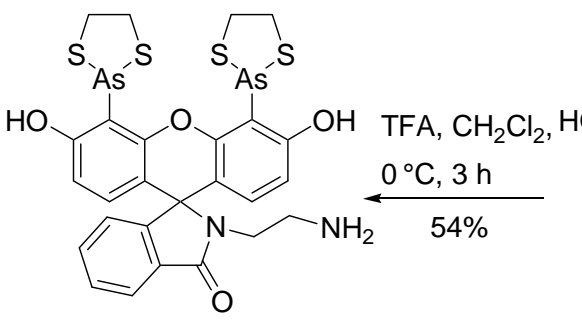

$105 a$
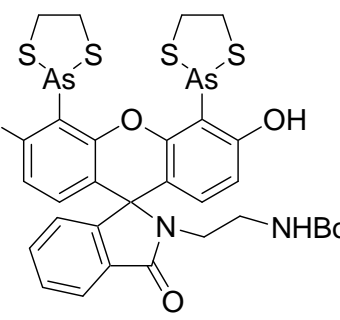

111
1) $\mathrm{AsCl}_{3}$, DIPEA, $\mathrm{Pd}(\mathrm{OAc})_{2}, \mathrm{THF}, 50^{\circ} \mathrm{C}, \mathrm{HO}$ $2 \mathrm{~h}$, then $\mathrm{r} . \mathrm{t}, 16 \mathrm{~h}$

2) 1,2-ethanedithiol, phosphate buffer, acetone, $30 \mathrm{~min}$ $44 \%$ overnight

Scheme 48. Synthesis of SplAsH 105a. ${ }^{[7,138]}$ 
The first precursor for the SplAsH-based biarsenical fluorescent probes - compound 105a - was prepared according to a published procedure $^{[7]}$ from fluorescein (compound 106). The methyl ester 107 was synthesized by esterification of fluorescein catalyzed by sulfuric acid. ${ }^{[138]}$ The ester $\mathbf{1 0 7}$ was isolated in 97\% yield, and it was then converted into the spirolactam 109 by amidation with the monoprotected 1,2-diaminoethane 108. Electrophilic substitution of the two aromatic protons with mercuric acetate gave the bis-mercuric spirolactam 110 in high yield (90\%). The catalytic transmetallation of compound $\mathbf{1 1 0}$ with arsenic chloride in the presence of palladium acetate followed by closure of the dithiarsolanic ring gave the Boc-protected SplAsH (compound 111) in moderate yield (44\% vs. $95 \%$ in the literature). Deprotection of compound 111 with trifluoroacetic acid resulted in SplAsH 105a.

Two of the three required dyes with an amino-reactive group ( $N$-hydroxysuccinimidyl ester), were prepared as described in the literature. ${ }^{[8,139,140]}$ Before that, the linker for binding the water-soluble rhodamine with SplAsH 105a was synthesized.

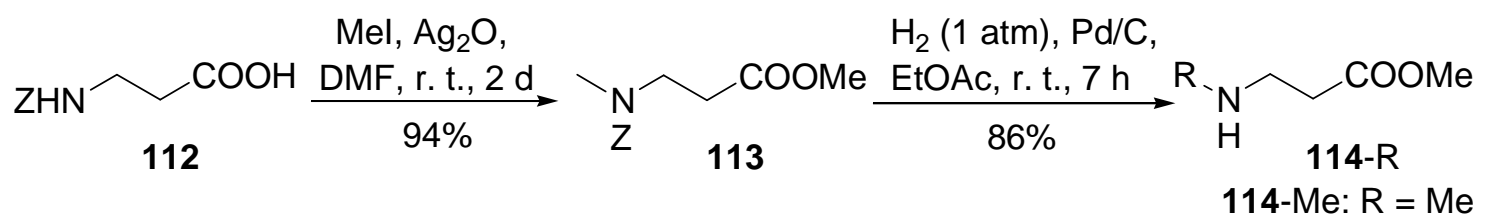

Scheme 49. Synthesis of the short bifunctional linker 114-Me.

As a starting material, the commercially available Z- $\beta$-alanine (112) was chosen. After twofold (N, O) methylation and cleavage of the Z-group by hydrogenolysis, methyl $N$-methyl$\beta$-alaninate (114-Me) was produced. ${ }^{[8]}$ The yields in both steps were high $(94 \%$ and $86 \%)$. 


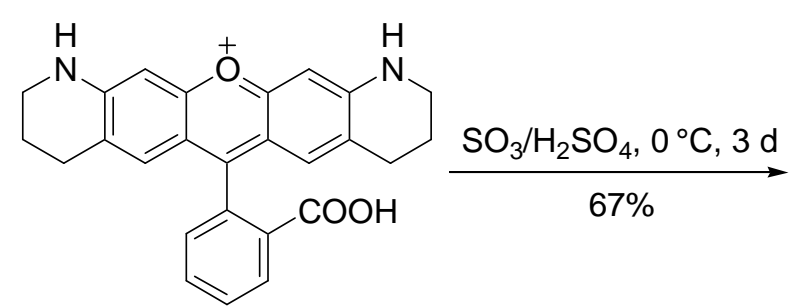

115

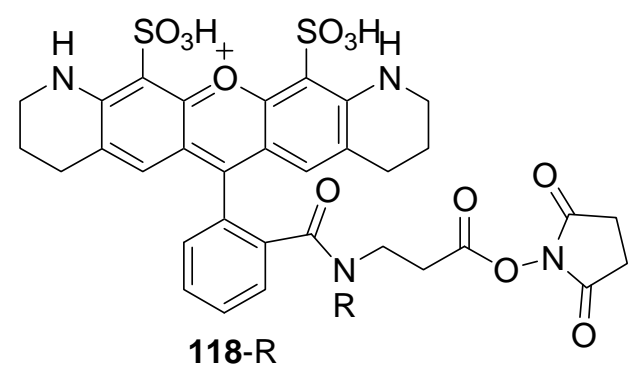

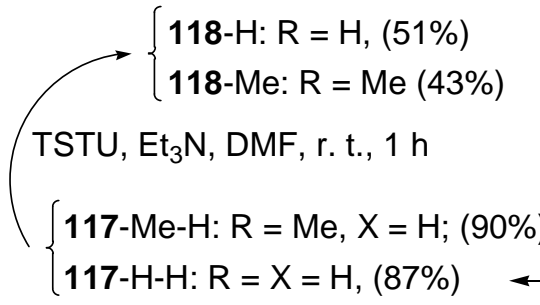<smiles>O=C(O)c1ccccc1-c1c2cc3c(c(S(=O)(=O)O)c2[o+]c2c(S(=O)(=O)O)c4c(cc12)CCCN4)NCCC3</smiles>

116 3 equiv. TSTU, DIPEA, DMAA, r. t., 2 h, then 114-R, overnight<smiles></smiles>

117-R-X

117-Me-Me: $\mathrm{R}=\mathrm{X}=\mathrm{Me} ;(60 \%)$

117-H-Me: $\mathrm{R}=\mathrm{H}, \mathrm{X}=$ Me. $(19 \%)$

Scheme 50. Synthesis of the amino-reactive derivatives of the water-soluble Rhodamine $\mathrm{S}$ (116) - compounds 118-R. ${ }^{[8,139,140]}$

For the synthesis of the NHS-ester of the fluorescent version of the water-soluble Rhodamine S 118-Me, ${ }^{[8]}$ rhodamine Q 115 was used as a starting material. The latter was mixed with $30 \%$ oleum in order to introduce two sulfonic acid groups. ${ }^{[8]}$ Thus, Rhodamine $\mathrm{S}$ $\mathbf{1 1 6}^{[8]}$ was produced in good yield $(67 \%)$. Then the $N$-hydroxysuccinimidyl ester was prepared from the carboxylic acid $\mathbf{1 1 6}$ in situ followed by the reaction with compound 114$\mathrm{Me}$, which afforded the methyl ester $117-\mathrm{Me}-\mathrm{Me}^{[8]}$ in good yield (60\%). The saponification of the methyl ester 117-Me-Me was carried out under mild conditions, producing the carboxylic acid 117-Me- $\mathrm{H}^{[8]}$ in high yield (90\%). Treatment of the acid 117-Me-H with TSTU provided the $N$-hydroxysuccinimidyl ester 118-Me. ${ }^{[8]}$ The preparative yield of this ester was moderate (43\% after purification by HPLC).

The non-fluorescent $N$-hydroxysuccinimidyl ester $118-\mathrm{H}^{[140]}$ was prepared in a similar way as compound 118-Me. Amidation of the in situ formed $N$-hydroxysuccinimidyl ester of the carboxylic acid $\mathbf{1 1 6}$ with the commercially available methyl ester of $\beta$-alanine (compound 114-H) afforded the methyl ester $117-\mathrm{H}_{-} \mathrm{Me}^{[139]}$ in low yield (18\%). After saponification of the methyl ester 117-H-Me under the same conditions as for compound 117-Me-Me, the trisodium salt was obtained and converted into the free acid $117-\mathrm{H}-\mathrm{H}^{[140]}$ by treatment with a 
strongly acidic ion-exchange resin in its $\mathrm{H}^{+}$-form. In the last step, the compound 118- $\mathrm{H}^{[140]}$ was isolated by HPLC as the product of the reaction between the acid 117-H-H and TSTU. The yield of the NHS-ester 118-H was moderate (51\%).

\section{Synthesis of reactive fluorescent labels}

\subsection{Synthesis of the water-soluble labels}

Initially, we planned to prepare the required compound SplAsH-RhS-OF by a reaction between NHS-ester 118-Me and the amine 105a in dichloromethane and in the presence of an organic base.<smiles>NCCN1C(=O)c2ccccc2C12c1ccc(O)c([As]3SCCS3)c1Oc1c2ccc(O)c1[As]1SCCS1</smiles>

$105 a$<smiles>[R]CCNC(=O)c1ccccc1-c1c2c(cc3c(S(=O)(=O)O)c4c(cc13)CCCN4)CCCN2</smiles>

$118-R$ $118-\mathrm{H}: \mathrm{R}=\mathrm{H}$ 118-Me: $\mathrm{R}=\mathrm{Me}$ $\mathrm{Et}_{3} \mathrm{~N}$ r. t., overnight<smiles>NCCN1C(=O)c2ccccc2C12c1ccc(O)c([As]3SCCS3)c1Oc1c2ccc(O)c1[As]1SCCS1</smiles>

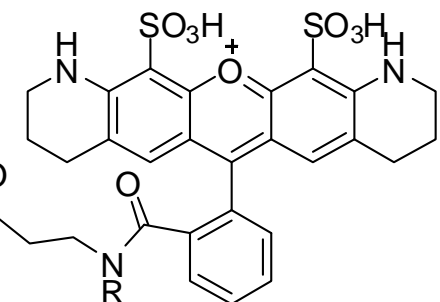

SpIAsH-RhS-OF: R = Me; (32\%) SplAsH-RhS-CF: R = H. (19\%)

Scheme 51. Preparation of two biarsenical probes with hydrophilic rhodamines.

However, due to the low solubility of compound 118-Me in dichloromethane, DMF was used instead. The target product was observed in this case, but the conversion was very low (only 7\%). A higher conversion (about 74\%) was achieved after optimization of the reaction conditions: the order of the addition of components, and the nature of solvents. The final product - compound SplAsH-RhS-OF - was difficult to isolate from the reaction mixture. It was found to be extremely unstable. Preparative TLC, as well as column chromatography was not suitable for purification (such methods were used for the isolation of the similar 
compounds ${ }^{[7]}$ ). The main reason for this unstability is probably the presence of the dithiarsolanic rings and the sulfonic acid residues in the same molecule. To protect the product SplAsH-RhS-OF, we needed to minimize its contact with silica gel. Therefore, reversed-phase HPLC was used for the isolation and purification. The product was successfully isolated, and its purity was found to be $89 \%$ (HPLC by area). However, during the evaporation of solvents (acetonitrile and water) in a freeze dryer, the purity went down to $29 \%$ (HPLC by area). The most obvious reason is the decomposition due to the presence of trifluoroacetic acid (TFA) in the eluate. To neutralize the TFA, we collected the eluate in solutions of the organic buffer - ammonium acetate. This helped us to save most of the isolated compound, and the content of product SplAsH-RhS-OF after freeze drying was found to be $85 \%$.

Another factor also decreased the overall yield; namely, the preparation of the reaction mixture for the HPLC isolation procedure, which first step requires evaporation of the solvents from the reaction mixture $\left(\mathrm{CH}_{2} \mathrm{Cl}_{2}, \mathrm{Et}_{3} \mathrm{~N}\right.$ and DMF). For a certain time, the product remains in the reaction mixture without a base, and in a base absence, the content of SplAsHRhS-OF drops from 74 to $17 \%$. In order to decrease the loss of the target compound during evaporation of the solvents, we minimized the applied amount of solvents in the reaction. As a result, the content of the target compound in the reaction mixture was found to be $33 \%$.

The last improvement in the purification procedure was achieved, when the temperature of the HPLC column was reduced to $6{ }^{\circ} \mathrm{C}$. As a result, the purity of the isolated product was increased to $96 \%$ (vs. $89 \%$ at $25^{\circ} \mathrm{C}$ ).

The second target compound - SplAsH-RhS-CF - was prepared and isolated similarly to SplAsH-RhS-OF. After the solvent removal, the reaction mixture contained $40 \%$ of the target compound (HPLC by area), and the purity of the isolated product SplAsH-RhS-CF was found to be $81 \%$ (HPLC by area).

\subsection{Synthesis of the TMR derivative intended for the localization of the tagged proteins in mitochondria}

The third fluorescent biarsenical probe had to have the heightened mitochondrial localization. Positively charged TMR-derivatives are known to label mitochondria. ${ }^{[141]}$ Thus, we decided to produce the compound SpIAsH-TAMRA (Scheme 52), which should be transported to the tagged mitochondria owing to the presence of a TMR moiety, and it should be bound to the tetracysteine tag in the mitochondrial proteins owing to the presence of a SplAsH component. 


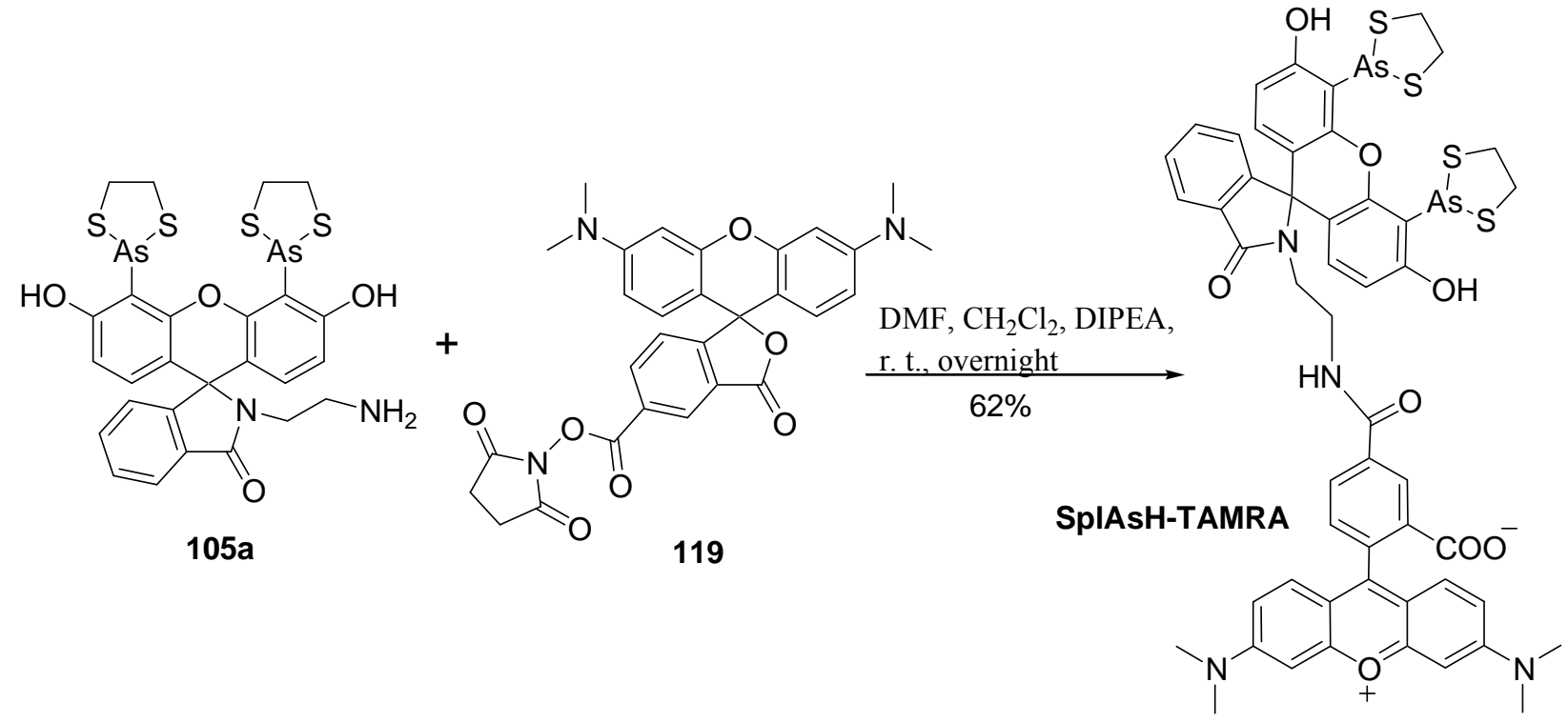

Scheme 52. Preparation of compound SplAsH-TAMRA.

We prepared the adduct from the compound 105a and the commercially available 6TAMRA,SE ${ }^{\circledR}$ (119) under similar conditions, as for compounds SplAsH-RhS-CF and SplAsH-RhS-OF described above. The isolation of the conjugate SplAsH-TAMRA was found to be much easier than the isolation of the hydrophilic analogues. In the absence of sulfonic acid residues in the molecule of SpIAsH-TAMRA the purification of this compound could be carried out by conventional column chromatography (on a reversed phase column).

\section{Spectroscopic control of the prepared biarsenical probes}

To confirm the binding ability of the target products (SplAsH-RhS-CF and SplAsHRhS-OF), we carried out competitive spectroscopic experiments. We investigated the binding abilities of the SplAsH derivatives (SplAsH-RhS-CF and SplAsH-RhS-OF) and compared them with the binding ability of FIAsH (compound 103a). In the first experiment, it was established that the position of the fluorescence maximum of compound SplAsH-RhS-OF was at $557 \mathrm{~nm}$.

In the second experiment, the position $(529 \mathrm{~nm})$ and the intensity of the fluorescence signal of a $1 \mu \mathrm{M}$ aqueous solution of compound 103a was determined. The reaction mixture which contained FIAsH and the decapeptide GSSGCCPGCC in a 1:1 ratio (both components in $1 \mu \mathrm{M}$ concentration) showed a five times higher fluorescence intensity. 


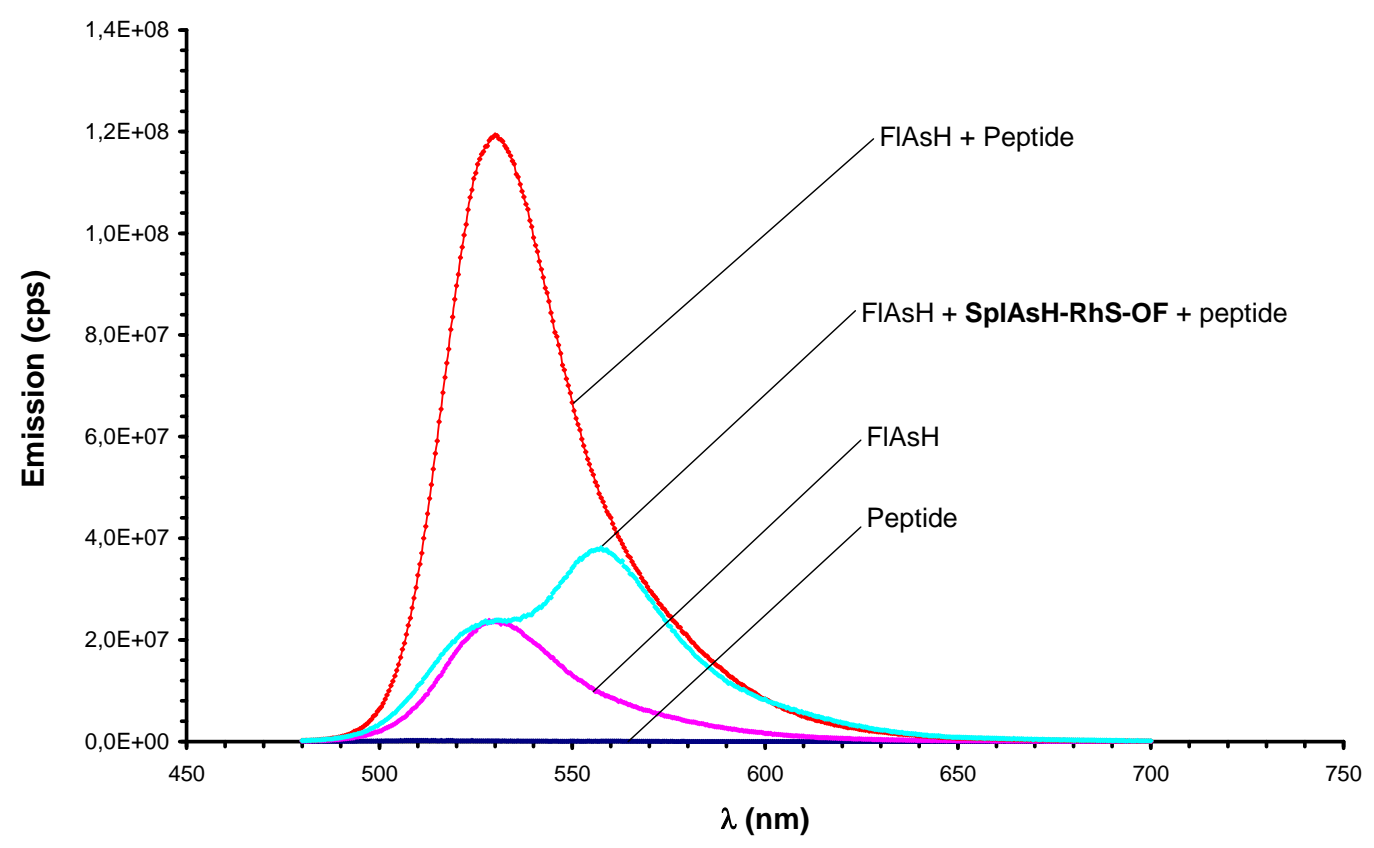

Figure 68. Comparison of the binding ability of SplAsH-RhS-OF and of FlAsH with the decapeptide GSSGCCPGCC: a) the dark base blue line represents the fluorescence signal of the peptide (almost non-fluorescent); b) the pink line corresponds to the fluorescence of a 1 $\mu \mathrm{M}$ aqueous solution of FlAsH, c) the red line displays the fluorescence signal of a 1:1mixture of FlAsH and the decapeptide (concentrations of the both components are the $1 \mu \mathrm{M}$ ), d) the light blue line represents the result of the competition experiment: FIAsH : SplAsHRhS-OF : peptide $=1: 1: 1$. The intensity of the fluorescence signal is measured in "cps" (counts per second).

The third fluorescence spectrum displays the emission signal of the three-component mixture - FIAsH, SplAsH-RhS-OF and the decapeptide - in a 1:1:1 equimolar ratio. These components were mixed in a competitive mode, i. e. the peptide was added to the mixture of FlAsH and SplAsH-RhS-OF in the buffer solution. As a result, the fluorescence spectra possessed two maxima which were attributed to FlAsH (529 nm) and to SplAsH-RhS-OF $(557 \mathrm{~nm})$. However, the weaker absolute intensity of the FIAsH band (than in the case of the pure FlAsH-peptide complex) can be attributed to the non-bound form of FIAsH (or to a mixture of the non-bound form and a small amount of the bound form). Unfortunately, the superposition of the two bands from the (unbound) FlAsH and SplAsH-RhS-OF did not allow us to estimate the quantitative parameters of the binding process.

Towards this goal, we used the compound SplAsH-RhS-CF that has a similar structure, but is not fluorescent at 400-600 $\mathrm{nm}$. As a consequence, in the competition experiment only a decrease in the intensity of the fluorescence maximum of FIAsH should be observed. 


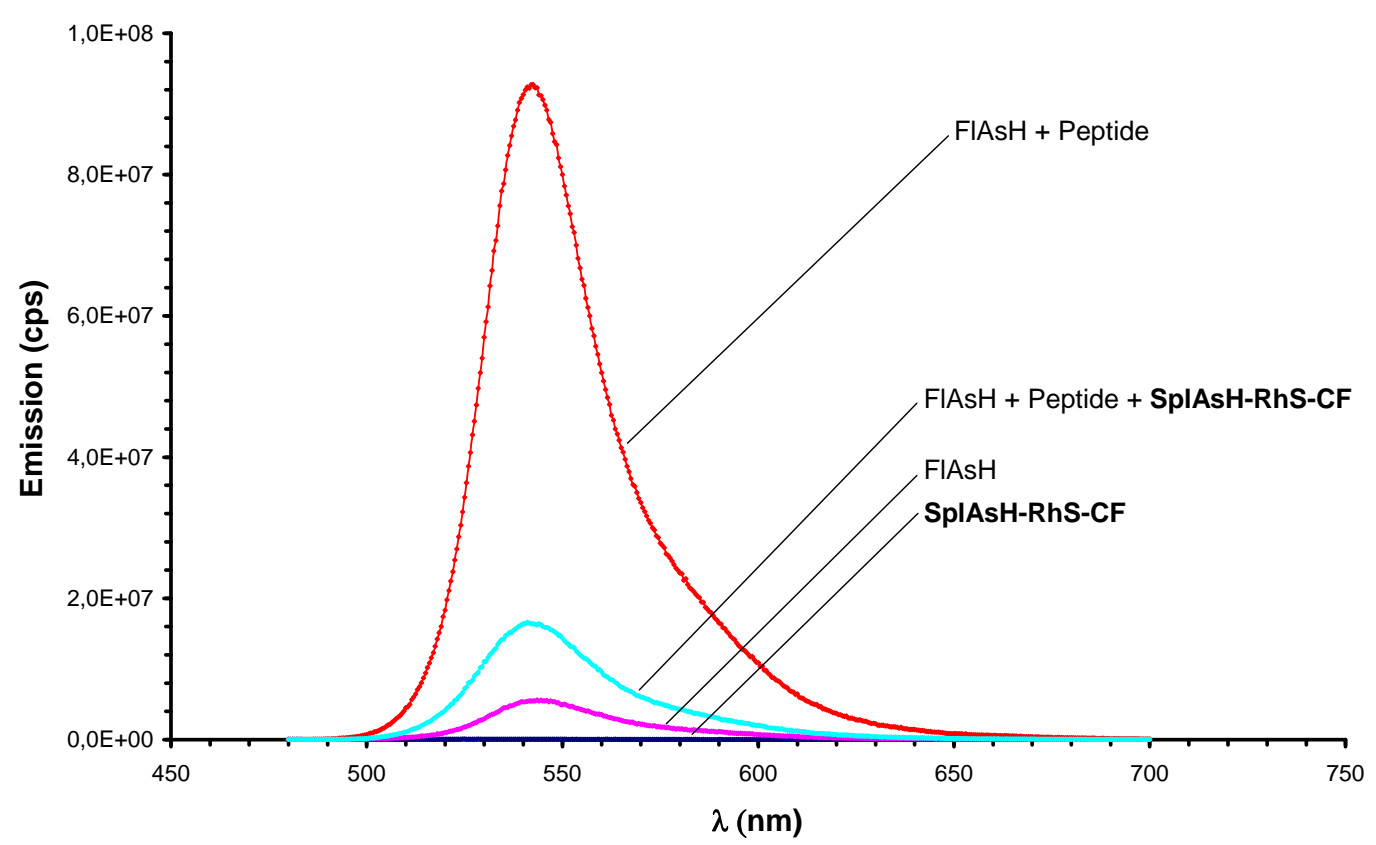

Figure 69. Comparison of the binding ability of SplAsH-RhS-CF and FlAsH with the decapeptide GSSGCCPGCC: a) the dark blue line represents the background fluorescence from SplAsH-RhS-CF (almost no emitted light could be seen); b) the pink line displays the fluorescence of the FlAsH solution, c) the red line gives the fluorescence intensity of a mixture of FlAsH and the decapeptide in a molar ratio of $1: 1$, d) the light blue line is a competition experiment (FIAsH: SplAsH-RhS-CF: peptide $=1: 1: 1$ ). The measure of the intensity of the fluorescence signal is "cps" (counts per second).

The presence of the two sulfonic acid residues in the structure of compound SplAsHRhS-CF did not substantially increase the solubility of this compound in water. Thus, for the measurements, we applied a mixture of the PBS buffer and DMF (in a volume ratio of 1:1). Under these conditions, the fluorescence maximum of FlAsH was shifted to $542 \mathrm{~nm}$. The intensity of the fluorescence signal of the mixture consisting of FIAsH and the decapeptide increased 16.4 times in comparison with a solution of the pure FIAsH in the same solvent mixture (although, binding of FIAsH with the same peptide in water produced only a 5-fold increase in the fluorescence signal intensity). The last fluorescence spectrum represents the emission of a three-component mixture of FIAsH, SplAsH-RhS-CF and the peptide in an equimolar ratio. These components were mixed in the following order: the peptide was added to the mixture of FIAsH and SplAsH-RhS-CF in aqueous DMF. The resulting fluorescence spectra possessed only one band at $542 \mathrm{~nm}$, which was 3 times more intensive than the fluorescence band of a solution of the pure FIAsH (without the peptide). If we denote the fraction of the unbound FIAsH in the mixture of the competition experiment as X, the bound 
FlAsH as 1-X and the fluorescence of the solution of pure FlAsH as A, then the fluorescence of the FlAsH-peptide complex is $16.4 * \mathrm{~A}$ and the fluorescence in the competition experiment is $3^{*} \mathrm{~A}$. Then from the equation $3^{*} \mathrm{~A}=\mathrm{X}^{*} \mathrm{~A}+16.4^{*} \mathrm{~A}(1-\mathrm{X})$ we calculate that $\mathrm{X}=13.4 / 15.4=$ 0.87. This means, that most of the FlAsH is in the non-bound state, and the ratio of the free FlAsH to the bound FlAsH is about 7:1. Thus, most of the decapeptide was bound with the non-fluorescent SplAsH derivate.

\section{Bioimaging tests of the prepared biarsenical probes}

Before any experiments with living cells could be conducted, it was necessary to check the binding ability of the SplAsH derivatives in vitro. Therefore, a TetCys-mut-GFP-TetCys protein was chosen as the test substrate. This represents an isolated Green Fluorescent Protein with two point mutations that disrupt its ability to emit light and with additional tetracysteine motifs attached to $N$ - and $C$-Terminals. 


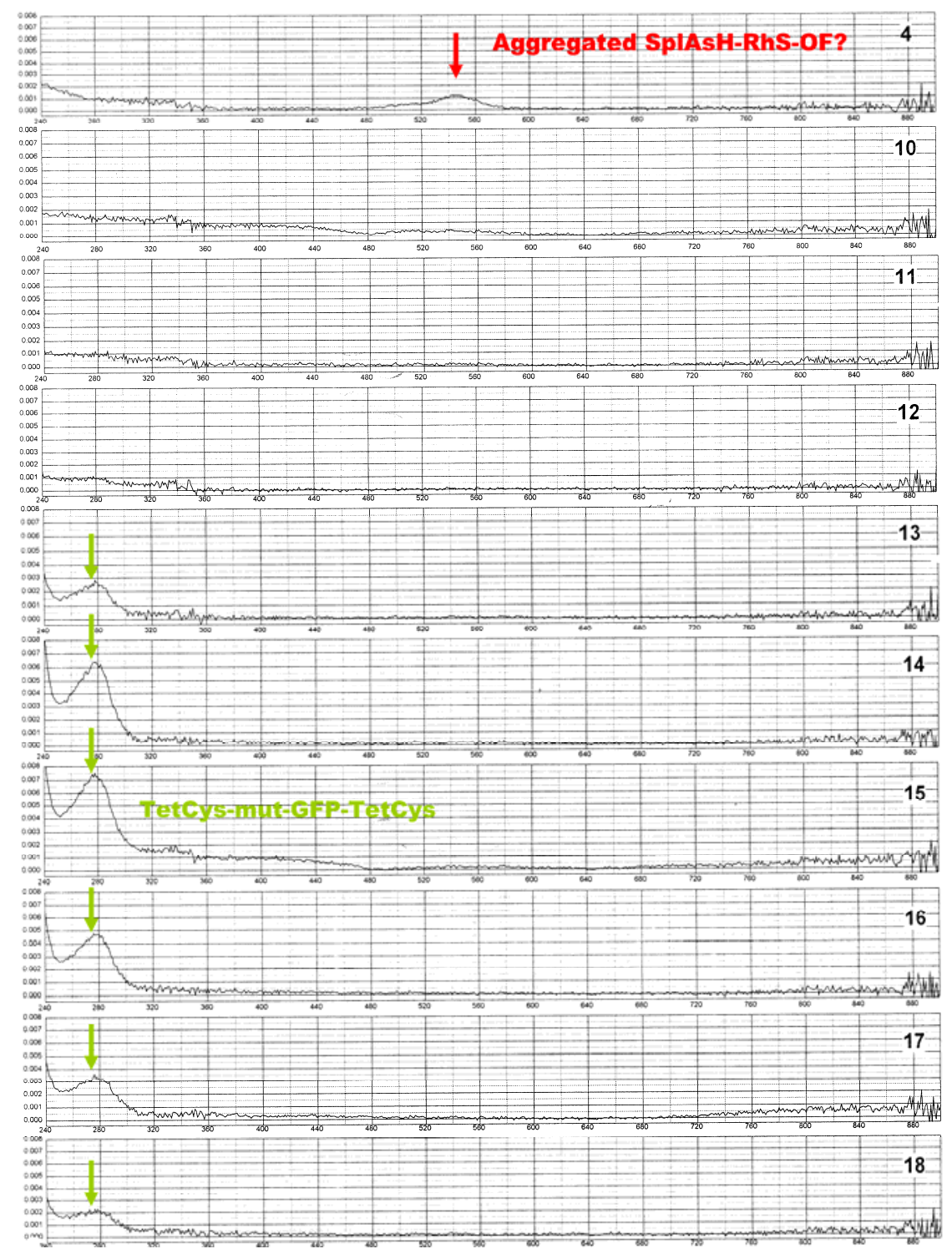

Figure 70. Absorption spectra of the collected fractions from size exclusion chromatography performed after incubation of TetCys-mut-GFP-TetCys-protein with the compound SplAsHRhS-OF. The numbers on the right side of the spectra are the fraction numbers (every fraction was $0.5 \mathrm{~mL}$ ). At the beginning we can observe the fractions which contain the unknown substrates (for example fraction number 4 has in the absorption spectrum a band at $550 \mathrm{~nm}$, that can indicate the presence of SplAsH-RhS-OF (or products of its decomposition) in this fraction). Then, in the absorption spectra of the fractions 13-18, where the substances with higher molecular weight should be present, a band at $280 \mathrm{~nm}$ was observed (this band is marked with green arrows). These fractions contain the TetCys-mut-GFP-TetCys-protein.

We tried to isolate the conjugate of the above mentioned protein with the compound SplAsH-RhS-OF using size exclusion chromatography with a spectroscopic control. Unfortunately, we did not find any fractions displaying the absorption bands of the protein 
TetCys-mut-GFP-TetCys (at $280 \mathrm{~nm}$ ) and the compound SplAsH-RhS-OF (at $550 \mathrm{~nm}$ ) (see Figure 70).

As a positive control, a similar experiment was done with the commercially available FlAsH (Lumio-Green/Invitrogen). In this experiment, the FlAsH was clearly bound to the tetracysteine containing protein (see Figure 71).

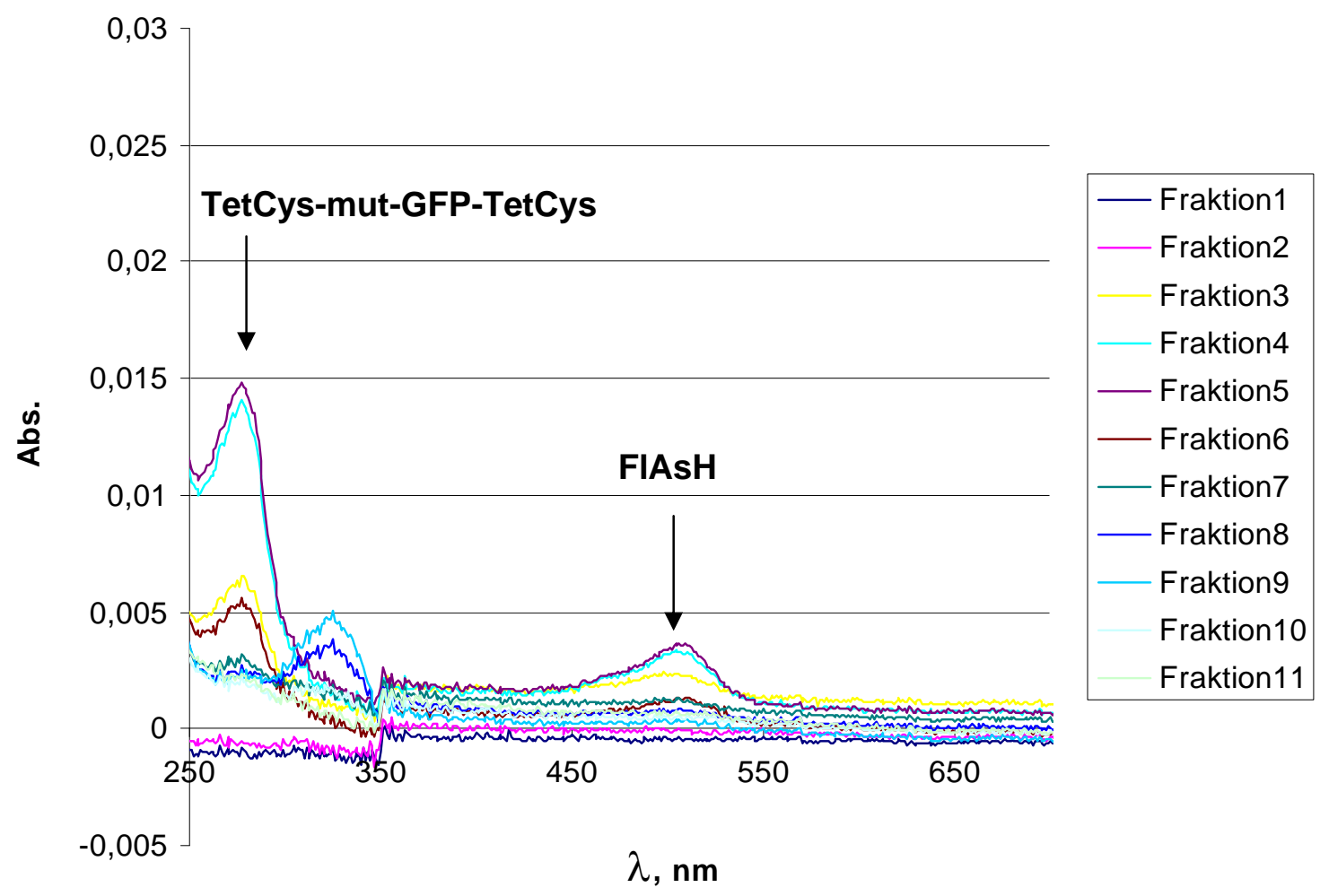

Figure 71. Absorption spectra of the collected fractions from the size exclusion chromatography column obtained after the conjugation experiment between TetCys-mutGFP-TetCys-protein and FIAsH. The fractions 4 and 5 both contain characteristic bands attributed to TetCys-mut-GFP-TetCys-protein, and to FlAsH; their maxima are marked with black arrows).

After that, we performed another conjugation experiment, in which a preliminary treatment of the protein with the TCEP reagent (for reduction of the disulfide bonds) was performed. As an analytical method, the sodium dodecyl sulfate polyacrylamide gel electrophoresis (SDS-PAGE) was chosen. 
A.

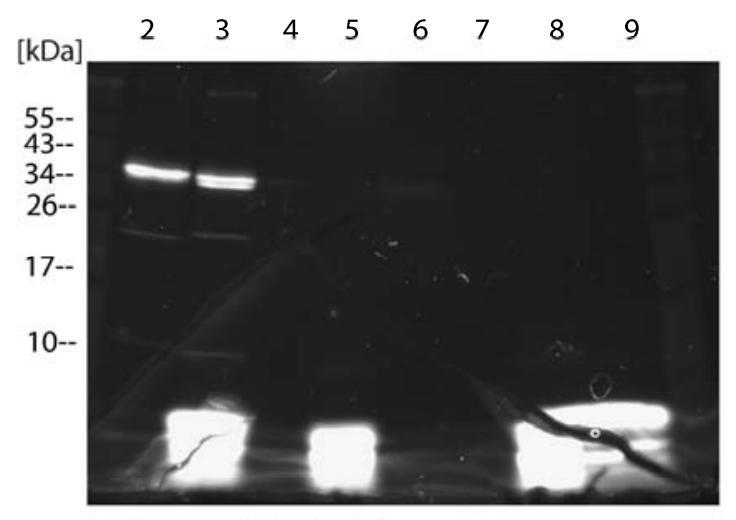

illuminated with UV
B.

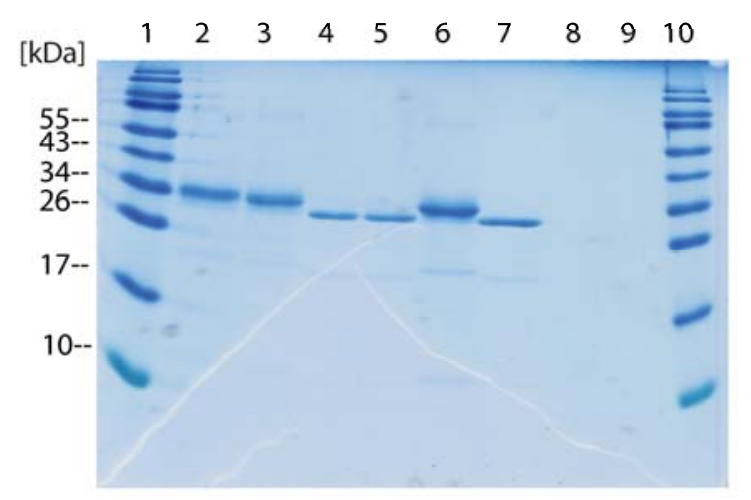

stained with Roti-Blue

Figure 72. SDS-PAGE of TetCys-mut-GFP-TetCys-protein labelled with FIAsH and the compound SplAsH-RhS-OF. The picture A is taken under UV light (left) and the picture B after staining with $\operatorname{Roti}^{\circledR}$-Blue (right). The number of lanes is the same in both pictures. By the special Roti ${ }^{\circledR}$-Blue dye we visualized the protein bands. Lanes $\mathbf{1}$ and $\mathbf{1 0}$ contain a set of molecular weight markers, lanes 8 and $\mathbf{9}$ contain big bands which are visible only under UV light and can be attributed to SplAsH-RhS-OF (and its decomposition products), 7 - the band of mutated non-fluorescent GFP, 6 - the band of TetCys-mut-GFP-TetCys-protein (also mutated GFP, but with two tetracysteine tags attached to the $N$ - and $C$-terminals), lane 5 corresponds to the experiment with mutated GFP and SplAsH-RhS-OF [there are two bands: the first one (on picture B) is attributed to the non-fluorescent mutated GFP, the second one (on picture A) is a big fluorescent band of SplAsH-RhS-OF that indicates the absence of binding of the biarsenical probe SplAsH-RhS-OF to the protein without a tetracysteine tag), 4 - the same experiment as in the lane 5, but the biarsenical probe FIAsH was used for the labelling (in this lane, only the band of the mutated GFP-protein is presented, because the non-bound FlAsH is non-fluorescent), 3 - the main experiment with TetCys-mut-GFPTetCys-protein and biarsenical probe SplAsH-RhS-OF (two fluorescent bands can be seen: the low band corresponds to the trace of the initial biarsenical probe SplAsH-RhS-OF, the upper band corresponds to the complex of TetCys-mut-GFP-TetCys-protein with the biarsenical probe SplAsH-RhS-OF, the protein nature of which was confirmed by the blue band in the left picture), 2 - additional control experiment with TetCys-mut-GFP-TetCysprotein and biarsenical probe FIAsH (only one fluorescent band is seen, namely the complex of TetCys-mut-GFP-TetCys-protein with the biarsenical probe FIAsH, the protein nature of which was confirmed by the blue band in the left picture). 
The presence of the bright fluorescent band (visible upon UV light illumination) in the expected area of the SDS-PAGE gel indicate, that a complex between TetCys-mut-GFPTetCys-protein and the compound SpIAsH-RhS-OF was formed and could be separated by the SDS-PAGE method.

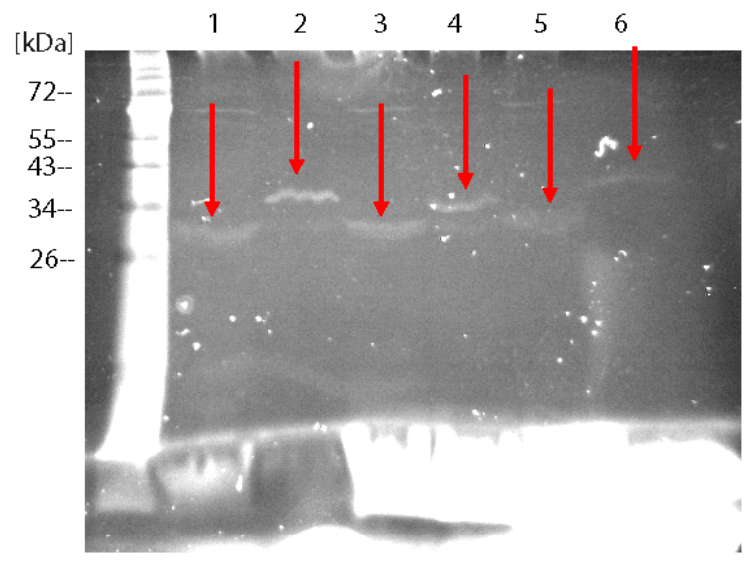

illuminated with UV

Figure 73. SDS-PAGE of isolated mitochondria from a yeast strain that expresses a triple tetracysteine tagged Porl protein and is labelled with compound SplAsH-RhS-OF and compound SplAsH-TAMRA (negative control - wild type mitochondria, positive control FlAsH). Lanes: 6 - experiment with the isolated mitochondria with the triple tetracysteine tagged version of Porl (one of the proteins of the outer mitochondrial membrane) and biarsenical probe SplAsH-RhS-OF (the red arrow points to the fluorescent band of the labelled mitochondrial protein that indicates the binding of the biarsenical probe SplAsHRhS-OF with the tetracysteine tagged version of the mitochondrial protein Por1), 5 experiment with wild-type mitochondria and SpIAsH-RhS-OF (the red arrow points to the very pale fluorescent band of the unspecifically labelled mitochondrial protein; the absence of the fluorescent band at the same place as in lane $\mathbf{6}$ confirms the binding ability of SplAsHRhS-OF), 4 - the same experiment as in the case of lane 6, but with biarsenical probe SpIAsH-TAMRA (the red arrow points to the fluorescent band of the labelled mitochondrial protein that indicates binding of the biarsenical probe SpIAsH-TAMRA to the tetracysteine tagged version of the mitochondrial protein Por1), 3 - experiment with a wild-type mitochondria and SpIAsH-TAMRA (the red arrow points to the very pale fluorescent band of the unspecifically labelled mitochondrial protein; the absence of the fluorescent band at the same place as in the lane $\mathbf{4}$ confirms the binding ability of SplAsH-TAMRA), 2 - additional control experiment with the triple tetracysteine tagged version of the mitochondrial protein Por1 and biarsenical probe FIAsH (the red arrow points to the fluorescent band of labelled 
mitochondrial protein that confirms the presence of the tetracysteine tags in the used mitochondrial protein Por1 to which the biarsenical probe FIAsH are bound), 1 - experiment with wild-type mitochondria and FlAsH (the red arrow points to the very pale fluorescent band of the unspecifically labelled mitochondrial protein; the absence of the fluorescent band at the same place as in lane $\mathbf{2}$ confirms the binding ability of FlAsH).

The labeling experiments with the isolated mitochondria expressing a three TetCystagged version of Porl (one of the proteins in the outer mitochondrial membrane), were also successful. These mitochondria were successfully labelled with the two biarsenical probes: SplAsH-RhS-OF and SplAsH-TAMRA. These facts were confirmed in the same manner as earlier, i. e. by observation of the bright fluorescent lanes upon UV light illumination in the required area of an SDS-PAGE plate (Figure 73).

Therefore, we have confirmed, that the specific binding of our SplAsH derivatives to the tetracysteine tags in proteins could indeed be achieved.

Unfortunately, the attempted in vivo labelling of the mitochondrial proteins containing tetracysteine tags failed to produce positive results.

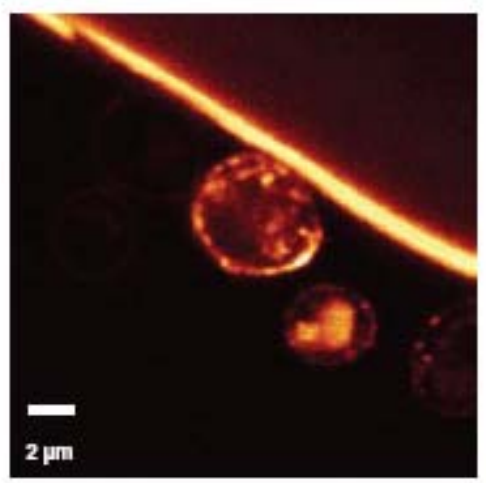

SplAsH-RhS

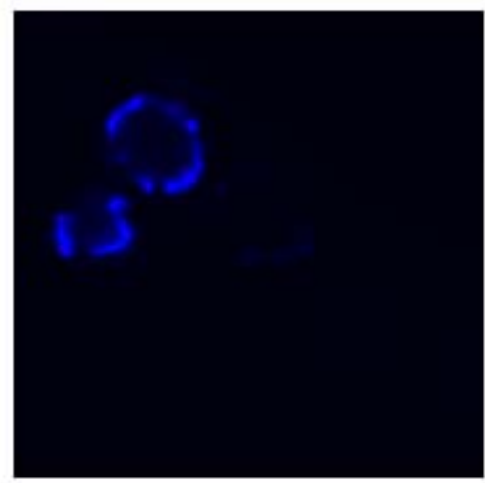

mBFP

Figure 74. Confocal image of yeast cells, stained by means of electroporation with SplAsHRhS-OF (left picture) and the recorded fluorescence of the same cells caused by the mitochondrial targeted Blue Fluorescent Protein (m-BFP) (right picture). In the right picture, we can see mitochondrial network (blue), but in the left picture we can see only unspecific background staining, because only random red spots inside the cells are seen.

A yeast strain expressing the mitochondrial targeted, mutated and tetracysteine tagged GFP and additional expressing a mitochondrial targeted Blue Fluorescent Protein was the first object of staining. These yeast cells were stained by electroshock according to a standard protocol $^{[142]}$ with FlAsH (as a positive control) or with SplAsH-RhS-OF. While the yeast 
mitochondria were very well stained with FIAsH, SplAsH-RhS-OF (see Figure 74) showed nearly no mitochondrial staining at all. A similar experiment which was carried out with SplAsH-TAMRA did not show any improvements compared to SplAsH-RhS-OF.

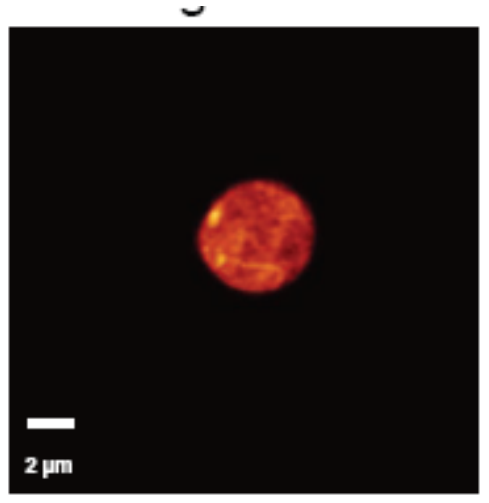

SplAsH-RhS

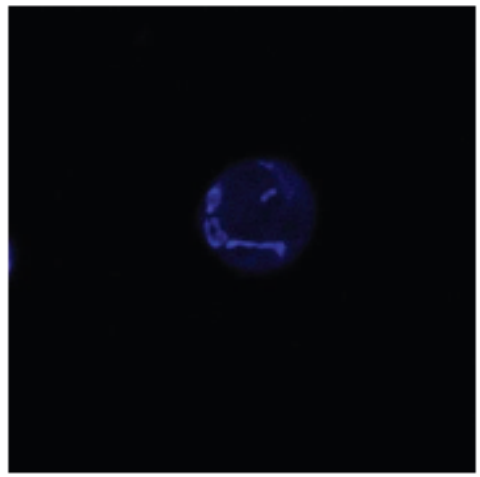

mBFP

Figure 75. Confocal image of PFA (polyformaldehyde) fixed yeast cells, which were permeabilized by treatment with Triton - X100 and stained with SplAsH-RhS-OF (left picture) and the detected fluorescence of the same cells from the mitochondrial targeted $\mathrm{m}$ BFP (right picture). In the right picture, we can see the bright blue mitochondrial network, which could be attributed to the cell mitochondria, but in the left picture we cannot see clear mitochondrial staining, because in this picture the entire cell is red-colored inside (without any separations of the organells).

The same yeast strain employed for the electrostaining was now fixed with $8 \%$ PFA and afterwards permeabilized with $0.5 \%$ Triton - X100. Neither SpIAsH-RhS-OF (Figure 75) nor SplAsH-TAMRA showed any significant degree of mitochondrial staining.

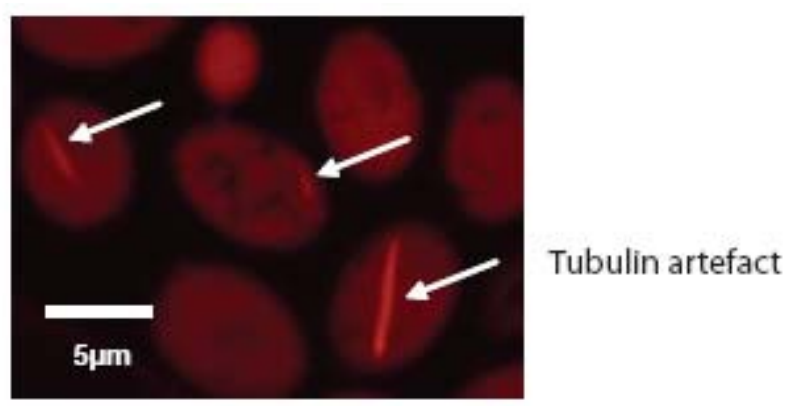

SpIAsH-TAMRA

Figure 76. Confocal image of the PFA-fixed yeast cells permeabilized by treatment with Triton - X100, treated with TCEP treatment and stained with the SpIAsH-TAMRA. In this 
picture, also the entire cells are red-colored inside (without any separations of the organelles, besides the strange tubulin-like artefacts, which look like a tubular structure inside the cells).

In the next series of experiments, the yeast cells expressing triple tetracysteine-tagged $\alpha$ Tubulin was used. Then, these cells were fixed and permeabilized as before, but additionally treated with $1 \mathrm{mM}$ TCEP to reduce disulfide bonds. They were afterwards stained with FlAsH, SplAsH-RhS-OF, or SplAsH-TAMRA (see Figure 76). As a negative control, a wild type strain was used. Unexpectedly, the TCEP treatment induced an $\alpha$-Tubulin-like staining artefact in the yeast cells regardless of the applied dye that can also be seen in wild-type cells.

Incubation of the TetCys-mut-GFP-TetCys expressing cells mentioned before with SplAsH-RhS-OF and SplAsH-TAMRA did not result in a clear mitochondrial staining, too.

Thus, higher numbers of tetracysteine tags in the one protein and the change of the type of protein in the combination with the TCEP pre-treatment also did not help to increase the selectivity of labelling by our biarsenical probes in vivo. 


\section{Experimental Part}

\section{General Remarks}

All chemicals obtained from commercial sources were used without additional purification. The catalyst, which was used for reduction of nitro compounds and debenzylation is $10 \% \mathrm{Pd} / \mathrm{C}$ (VWR International, oxidized form).

All reactions performed according the General Procedures 1, 3, 4 and 5 (GP1, GP3, GP4, GP5) were carried out in dry Schlenk flasks which were evacuated and flushed with argon three times.

NMR spectra were recorded on Varian Unity 300 and Varian Mercury $300(300 \mathrm{MHz}$ for ${ }^{1} \mathrm{H}, 75.5 \mathrm{MHz}$ for $\left.{ }^{13} \mathrm{C}\right)$, Varian INOVA $500\left(500 \mathrm{MHz}\right.$ for ${ }^{1} \mathrm{H}$ and $125.7 \mathrm{MHz}$ for $\left.{ }^{13} \mathrm{C} \mathrm{NMR}\right)$ or Varian INOVA $600\left(600 \mathrm{MHz}\right.$ for ${ }^{1} \mathrm{H}$ NMR) spectrometers in $\mathrm{CDCl}_{3}$ if not otherwise specified. Assingments of the ${ }^{13} \mathrm{C}$-signals to $\mathrm{CH}_{3}(\mathrm{CH})$ and $\mathrm{CH}_{2}\left(\mathrm{C}_{\mathrm{q}}\right)$ groups were made by APT (Attached Proton Test) measurements. Chemical shifts $(\delta)$ are given in ppm. All spectra are referenced to tetramethylsilane as an internal standard ( $\delta=0 \mathrm{ppm})$ using the signals of the residual protons of deuterated solvents: $\delta=7.26 \mathrm{ppm}$ for $\mathrm{CHCl}_{3}, 3.31 \mathrm{ppm}$ for $\mathrm{CHD}_{2} \mathrm{OD}$ and $2.50 \mathrm{ppm}$ for $\left[\mathrm{D}_{5}\right] \mathrm{DMSO}$. Multiplicities of the proton signals are described as follows: $\mathrm{s}=$ singlet, $\mathrm{d}=$ doublet, $\mathrm{t}=$ triplet, $\mathrm{q}=$ quartet, quint $=$ quintet, $\mathrm{m}=$ multiplet, $\mathrm{br}=$ broad. Coupling constants $(J)$ are given in Hz.

Melting points (uncorrected) were determined in capillaries using a SMP 10 apparatus (Bibby Sterling LTD, UK).

Electron ionization mass spectra (EI-MS, $70 \mathrm{eV}$ ) and chemical ionisation mass spectra (CI-MS, $200 \mathrm{eV}$, reactant gas $\mathrm{NH}_{3}$ ) were measured with a Finnigan MAT 95 spectrometer. Electrospray ionization mass spectra (ESI-MS) were recorded with a LCQ Finnigan spectrometer. High-resolution mass spectra (HR-MS) were measured with Bruker APEX IV $7 T$ FT-ICR instrument, and with ESI-TOF spectrometer microTOF (Bruker) equipped with the "Apollo" ion source.

Absorption and fluorescence stationary measurements were carried out with Varian Cary 4000 UV-Vis spectrophotometer, and Varian Cary Eclipse fluorescence spectrophotometer, respectively. Some of the fluorescence measurements were carried out using Fluorolog 3-22 (HORIBA Jobin Yvon GmbH, Unterhaching, Germany) emission spectrometer with double monochromator for the excitation and emitted light. The quartz cuvettes with 0.1 and $1 \mathrm{~cm}$ path lengths were used. Emission spectra were corrected for the instrument response. Irradiation of the compound $\mathbf{4 0}-\mathrm{H}$ to drive the photochromic reactions was performed with a 
200W Mercury lamp (LOT-Oriel GmbH \& Co. KG, Darmstadt, Germany) equipped with a monochromator and band-pass filter centered at $366 \mathrm{~nm}$ (UG1 - $3 \mathrm{~mm}$, Schott).

HPLC-System (Knauer): Smartline pump 1000 (2×), UV-detector 2500, column thermostat 4000, mixing chamber, injector with 20 and $100 \mu \mathrm{L}$ loops for the analytical and preparative columns, respectively; 6-port-3-channel switching valve; analytical column: Eurosphere-100 C18, $5 \mu \mathrm{m}, 250 \times 4 \mathrm{~mm}$; preparative column: Eurosphere-100 C18, $5 \mu \mathrm{m}$, $250 \times 8 \mathrm{~mm}$; solvent A: $\mathrm{H}_{2} \mathrm{O}+0.1 \% \mathrm{v} / \mathrm{v}$ TFA, solvent $\mathrm{B}: \mathrm{MeCN}+0.1 \% \mathrm{v} / \mathrm{v}$ TFA; temperature $25 \mathrm{C}$ (if not specified otherwise).

Analytical TLC was performed on aluminium plates precoated with silica gel $60 / \mathrm{F}_{254}$ (VWR International GmbH, Darmstadt, Germany), and on glass plates precoated with silica gel RP-18W/F 254 (Macherey-Nagel, Düren, Germany). Spots were visualized under ultraviolet light $(254 \mathrm{~nm})$ and / or developed by treatment with molybdenum phosphoric acid solution $(5 \%$ in $\mathrm{EtOH})$, aq. $\mathrm{KMnO}_{4}$ or iodine vapors. Flash chromatography was performed using VWR $\mathrm{SiO}_{2}$, grade 60, 0.04-0.063 mm. Reversed phase chromatography was performed on Polygoprep 60-50 C18 (Macherey-Nagel, Düren, Germany).

Anhydrous THF was destilled over sodium benzophenone ketyl. Organic solutions were dried with $\mathrm{MgSO}_{4}$ or $\mathrm{Na}_{2} \mathrm{SO}_{4}$. All reactions were carried out with magnetic stirring under positive pressure of argon or nitrogen using the standard vacuum - inert gas manifold, unless stated otherwise.

In the section 3.4 of Chapter 2 all measurements of the absorption and fluorescence spectra at various $\mathrm{pH}$ values of aqueous buffers were carried out after waiting period for $12 \mathrm{~h}$ after preparation of solutions. Other spectroscopic measurements in this section were carried out without waiting period.

For all experiments in the section 4 of Chapter 3: a) with compound SplAsH-RhS-OF was used $1 \times$ PBS buffer with addition of 2-mercaptoethanol $(1 \mathrm{mM})$; b) with compound SplAsH-RhS-CF was used the mixture $0.2 \times$ PBS buffer and DMF in the ratio $1: 1$ with addition of 2-mercaptoethanol $(1 \mathrm{mM})$; the fluorescence spectra were measured after waiting period for 12 h. $1 \times$ PBS buffer mixture: $137 \mathrm{mM} \mathrm{NaCl}, 2.68 \mathrm{mM} \mathrm{KCl}, 10.0 \mathrm{mM} \mathrm{Na} 2 \mathrm{HPO}_{4}$, $1.76 \mathrm{mM} \mathrm{KH}_{2} \mathrm{PO}_{4}$.

\section{Preparation of Known Compounds}

2-(4-(N,N-Diethylamino)-2-hydroxybenzoyl)-benzoic acid (37) and $N, N$-diethylrhodol 39-H were synthesized as described for the similar compounds. ${ }^{[93]} 1$-Aza-2,3-benzo-15- 
crown-5 (45) was prepared according to the published procedure. ${ }^{[96]} 2-[2-[2-(2-$ Chloroethoxy)-ethoxy]-ethoxy]-ethanol (53) was prepared according to Amabilino. ${ }^{[98]} 1,4-$ Bis(benzyloxy)benzene (56), 1,4-bis(benzyloxy)-2-nitrobenzene (57) and 4-(benzyloxy)-2nitrophenol (58) were synthesized according to Grynkiewicz. ${ }^{[35 b]} N$-(3-Methoxyphenyl)1,4,7,10-tetraoxa-13-azacyclopentadecane (67) was prepared by a procedure of BuchwaldHartwig amination. ${ }^{[103]}$ 2-(2,4-Dihydroxybenzoyl)-benzoic acid (69) was synthesized according to the published procedure. ${ }^{[64]}$ 4-Methoxy-5-nitrophthalic anhydride (74) and 4,5dinitrophthalic acid (78) were prepared according to the published procedure. ${ }^{[106]} 4$ Methoxyphthalic acid (81) was prepared from 3,4-dimethylphenyl methyl ether $\mathbf{8 0}$ according to the published oxidation method. ${ }^{[108]} 4,5$-Dichlorophthalic acid anhydride (86) and 4hydroxy-5-nitro- $N$-methylphthalimide (88) were synthesized according to Caswell. ${ }^{[111]} 4,5$ Dichloro- $N$-methylphthalimide (87) was prepared according to the published procedure. ${ }^{[12]}$ Potassium salt of 5-hydroxy-2-methyl-6-nitro-1 $H$-isoindole-1,3(2H)-dione (89) was synthesized according to the published procedure. ${ }^{[113]}$ 1,11-Diiodo-3,6,9-trioxaundecane (91) was prepared according to the published procedure. ${ }^{[114 \mathrm{a}]}$ 2-[2-[2-(2-Trityloxyethoxy)-ethoxy]ethoxy]-ethanol (94) and 2-[2-[2-(2-trityloxyethoxy)-ethoxy]-ethoxy]-ethyl mesylate (95) were synthesized according to Pilkington-Miksa. ${ }^{[16]}$ Methyl ester of fluorescein (107) was prepared according to the published procedure. ${ }^{[138]}$ Fluorescein spiroamide 109, its mercury derivative (110), Boc-protected SplAsH (111) and SplAsH (105a) were synthesized according to Bhunia. ${ }^{[7]}$ Methyl ester of $N$-benzyloxycarbonyl- $N$-methyl- $\beta$-alanine (113) and methyl ester of $N$-methyl- $\beta$-alanine (114-Me) were prepared according to the published procedures, Rhodamine S (116), the methyl ester 117-Me-Me, the carboxylic acid 117-Me-H and its $N$ hydroxysuccinimidyl ester 118-Me were synthesized according to the published procedures. ${ }^{[6]}$ The methyl ester 117-H-Me was prepared according to Belov. ${ }^{[139]}$ Acid $117-\mathrm{H}-\mathrm{H}$ and the NHS-ester 118-H were synthesized according to Polyakova. ${ }^{[140]}$
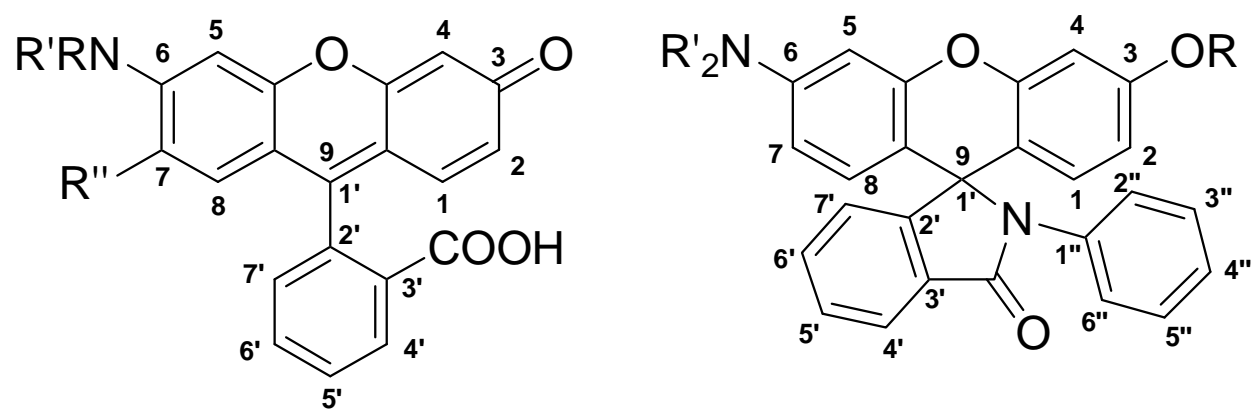

Figure 77. Numbering of rhodols (39-Me, Ia, Ib, Ic, Ic-Me•2/3TsOH, 70) and rhodolspiroamides (47-H, 47-Me). 


\section{Experimental procedures}

General procedure for the synthesis of xanthenes (35, Ia, Ib, Ic•2/3TsOH, 70) (GP1): A mixture of the corresponding phthalic anhydride $(1 \mathrm{mmol})$ or benzophenone $(1.5 \mathrm{mmol})$ and $p$-toluene sulfonic acid monohydrate $(8-12 \mathrm{~mol} \%)$ was loaded into the flask equipped with a reflux condenser and a bubble counter. Then the solution of $m$-aminophenol $(1.5 \mathrm{mmol}$ in the case of reaction with phthalic anhydride and $1.0 \mathrm{mmol}$ in the case of reaction with benzophenone) in propionic acid (1.5-6 mL) was added. The reaction mixture was heated at $150{ }^{\circ} \mathrm{C}$ for $24-48 \mathrm{~h}$ under argon or nitrogen. The solvent was evaporated in vacuo and the residue was purified by chromatography on silica gel.

General procedure for the reduction of a nitro group (the synthesis of compounds 48a, 97, 100) (GP2): The $10 \% \mathrm{Pd} / \mathrm{C}(20 \mathrm{w} / \mathrm{w} \%$ to the amount of the substance to be reduced $)$ and $\mathrm{MeOH}$ (THF, EtOAc) (5-15 mL) were placed into the Schlenk-flask equipped with septa. The mixture was flushed with hydrogen and stirred vigorously to activate the catalyst. A solution of the nitro compound $(1 \mathrm{mmol})$ in $\mathrm{MeOH}$ (THF, EtOAc) $(5-10 \mathrm{~mL})$ was introduced through a septum. The solution was stirred for $4-8 \mathrm{~h}$ at room temperature using the balloon with hydrogen. Then the hydrogen gas was replaced by argon, and the mixture was filtered through Celite $^{\circledR}$. The filter-cake was washed with $\mathrm{MeOH}$ (THF, EtOAc) $(3 \times 20 \mathrm{~mL})$ and the solvent was removed in vacuo. The product was purified by chromatography on silica gel.

General procedure for the monotosylation of alcohols (the synthesis of compound 101) and ditosylation of aminoalcohols (the synthesis of compounds 61, 98) (GP3): To a solution of alcohol $(1 \mathrm{mmol})$ or aminoalcohol $(1 \mathrm{mmol})$ in a dry pyridine $(5 \mathrm{~mL})$, tosyl chloride $(1.5-2.05 \mathrm{mmol})$ was added in small portions at $0{ }^{\circ} \mathrm{C}$, and the reaction mixture kept at $4{ }^{\circ} \mathrm{C}$ for $3-4$ days. Then it was poured onto the mixture of crushed ice $(20 \mathrm{~mL})$ and concentrated hydrochloric acid $(6 \mathrm{~mL})$. The mixture was warmed-up to room temperature and extracted with $\mathrm{CH}_{2} \mathrm{Cl}_{2}(3 \times 20 \mathrm{~mL})$. The combined organic solutions were washed with brine $(50 \mathrm{~mL})$, dried and concentrated in vacuo. The residue was purified by chromatography on silica gel.

General procedure for the crown ether ring closing (the synthesis of compounds 62, 63a, 99) (GP4): A solution of aminotosylate $(1 \mathrm{mmol})$ or ditosylate $(1 \mathrm{mmol})$ in dry DMF $(4-10$ $\mathrm{mL})$ was prepared in the Schlenk-flask equipped with a septum. Caesium carbonate (1.05 mmol) was added to the flask at room temperature, and the reaction mixture was heated at 90 
${ }^{\circ} \mathrm{C}$ for 24-39 hours. The solvent was evaporated in vacuo, and the residue was diluted with $\mathrm{CHCl}_{3}(30 \mathrm{~mL})$ and filtered through Celite. ${ }^{\circledR}$ The filter-cake was washed with $\mathrm{CHCl}_{3}(3 \times 10$ $\mathrm{mL}$ ), and the solvent was removed in vacuo. The residue was purified by chromatography on silica gel.

General procedure for debenzylation (the synthesis of compounds 42a, 42b, 42c) (GP5): $10 \% \mathrm{Pd} / \mathrm{C}(20 \mathrm{w} / \mathrm{w} \%$ to the amount of the substance to be debenzylated $)$ and $\mathrm{MeOH}(4 \mathrm{~mL})$ or AcOH (1-3 mL) were placed into a Schlenk-flask equipped with a septum. The mixture was flushed with hydrogen and stirred vigorously to activate the catalyst. A solution of benzyl ether $(1 \mathrm{mmol})$ in $\mathrm{MeOH}(8 \mathrm{~mL})$ or $\mathrm{AcOH}(2-3 \mathrm{~mL})$ was introduced through a septum. The solution was hydrogenated overnight at room temperature under hydrogen filled into a balloon attached to the reaction flask. Then the hydrogen gas was replaced by argon, and the mixture was filtered through Celite ${ }^{\circledR}$. The filter-cake was washed with $\mathrm{MeOH}$ or $\mathrm{AcOH}(3 \times 20$ $\mathrm{mL}$ ), and the solvents were removed in vacuo. Then the obtained oil was diluted with $\mathrm{CH}_{2} \mathrm{Cl}_{2}$ and the solvent was evaporated once more. The latter operation was repeated two times. The product was isolated as a brownish oil which oxidized easily in air.

\section{General procedure for the syntheses of the SplAsH adducts (SplAsH-RhS-OF, SplAsH-}

RhS-CF) (GP6): To a solution of an NHS-ester of the rhodamine derivative (7-8 $\mu \mathrm{mol})$ and $\mathrm{Et}_{3} \mathrm{~N}(21 \mu \mathrm{mol})$ in dry DMF $(1 \mathrm{~mL})$, the solution of compound 105a $(21 \mu \mathrm{mol})$ in $\mathrm{CH}_{2} \mathrm{Cl}_{2}(0.4$ $\mathrm{mL}$ ) was added via a syringe, and the reaction mixture was stirred at room temperature overnight. Then all volatile materials were removed in vacuo (without heating). The residue was purified by reversed-phase preparative HPLC with $\mathrm{MeCN} / \mathrm{H}_{2} \mathrm{O}$ mixture (40:60 $\rightarrow$ 100:0, $\left.25 \mathrm{~min}, 7^{\circ} \mathrm{C}\right)$. The fractions were collected into the test tubes. Every tube $(\mathrm{V}=8 \mathrm{~mL})$ contained the cold $\left(4^{\circ} \mathrm{C}\right)$ aqueous solution of $\mathrm{AcONH}_{4}(25 \mu \mathrm{L}, 21 \mathrm{M})$. After filling of a tube, its content was immediately transferred into the cold flask $\left(-78^{\circ} \mathrm{C}\right)$. Then the solvent and $\mathrm{AcONH}_{4}$ were removed by freeze drying. The product was isolated as a rosy solid, which could be stored at $-20{ }^{\circ} \mathrm{C}$ for a long time (several months).

3-(N,N-Diethylamino)-4-methoxyaniline (33): 3-(N,N-diethylamino)-4-methoxyacetanilide

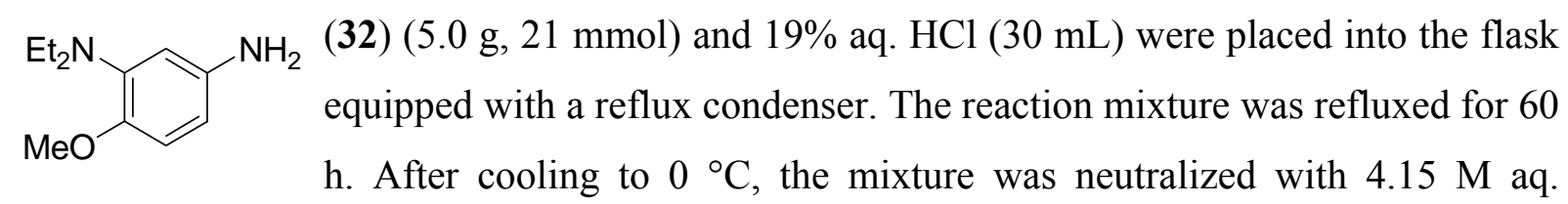
$\mathrm{NaOH}(50.7 \mathrm{~mL})$ and was extracted with $\mathrm{CHCl}_{3}(4 \times 80 \mathrm{~mL})$. The combined organic solutions 
were dried and concentrated in vacuo. The residue was purified by chromatography on silica gel (200 g) with $\mathrm{CH}_{2} \mathrm{Cl}_{2} / \mathrm{MeOH}$ mixture (20:1) as an eluent, and the title compound was isolated as a brown oil which easily oxidized in air (3.26 g, 80\%). ${ }^{1} \mathrm{H}$ NMR (300 MHz): $\delta=$ $1.01\left(\mathrm{t},{ }^{3} J=7.2,6 \mathrm{H}\right), 3.10$ (q, $\left.{ }^{3} J=7.2,4 \mathrm{H}\right), 3.35$ (br. s, $2 \mathrm{H}, \mathrm{NH}_{2}$ ), 3.76 (s, $3 \mathrm{H}, \mathrm{OMe}$ ), 6.27 $\left(\mathrm{dd},{ }^{3} J=7.8,{ }^{4} J=2.4,1 \mathrm{H}\right), 6.34\left(\mathrm{~d},{ }^{4} J=2.4,1 \mathrm{H}\right), 6.67\left(\mathrm{~d},{ }^{3} J=7.8,1 \mathrm{H}\right) \mathrm{ppm}$.

3-(N,N-Diethylamino)-4-methoxyphenol (31): Compound 33 (1.0 g, $5.2 \mathrm{mmol})$ was $\mathrm{Et}_{2} \mathrm{~N} \mathrm{OH}$ dissolved in $1.5 \mathrm{M}$ aq. $\mathrm{H}_{2} \mathrm{SO}_{4}(6.5 \mathrm{~mL})$, cooled to $0{ }^{\circ} \mathrm{C}$, and the solution of $\mathrm{NaNO}_{2}(0.44 \mathrm{~g}, 6.4 \mathrm{mmol})$ in water $(1 \mathrm{~mL})$ was added carefully dropwise.

The mixture was stirred for $10 \mathrm{~min}$. Then the several crystals of urea were added. The solution of diazonium hydrogen sulfate 34 was heated at $100{ }^{\circ} \mathrm{C}$ for $6 \mathrm{~h}$. After cooling to $0{ }^{\circ} \mathrm{C}$, the mixture was neutralized with $1 \mathrm{M}$ aq. $\mathrm{NaOH}$ and was extracted with $\mathrm{CH}_{2} \mathrm{Cl}_{2}(3 \times 40 \mathrm{~mL})$. The combined organic solutions were dried and concentrated in vacuo. The residue was purified by chromatography on silica gel $(100 \mathrm{~g})$ with $\mathrm{CH}_{2} \mathrm{Cl}_{2} / \mathrm{MeOH}$ mixture (20:1) as an eluent, and the title compound was isolated as a brownish solid, which easily oxidized in air $(0.35 \mathrm{~g}, 34 \%)$; m. p. $86{ }^{\circ} \mathrm{C}$ (hexane/ $\left.\mathrm{CH}_{2} \mathrm{Cl}_{2}\right) .{ }^{1} \mathrm{H}$ NMR $(300 \mathrm{MHz}): \delta=$ $0.96\left(\mathrm{t},{ }^{3} J=7.2,6 \mathrm{H}\right), 3.07\left(\mathrm{q},{ }^{3} J=7.2,4 \mathrm{H}\right), 3.72(\mathrm{~s}, 3 \mathrm{H}, \mathrm{OMe}), 6.40\left(\mathrm{dd},{ }^{3} J=9.0,{ }^{4} J=3.0\right.$, $1 \mathrm{H}), 6.46\left(\mathrm{~d},{ }^{4} J=3.0,1 \mathrm{H}\right), 6.65\left(\mathrm{~d},{ }^{3} J=9.0,1 \mathrm{H}\right) \mathrm{ppm} .{ }^{13} \mathrm{C} \mathrm{NMR}(75.5 \mathrm{MHz}): \delta=11.8$ $\left(2 \times \underline{\mathrm{CH}_{3}} \mathrm{CH}_{2}\right), 45.8\left(2 \times \mathrm{CH}_{3} \underline{\mathrm{CH}_{2}}\right), 55.9\left(\mathrm{CH}_{3} \mathrm{O}\right), 108.4(\mathrm{CH}), 109.6(\mathrm{CH}), 112.5(\mathrm{CH}), 139.9$ (C), 147.7 (C), 149.8 (C) ppm. EI-MS, m/z (rel. int., \%): 195 (54) [M] $]^{+}, 180$ (100) [M $\left.\mathrm{CH}_{3}\right]^{+}, 164$ (13), 151 (27), 136 (15). HR-MS (ESI) negative mode: found: 194.1188; calcd. for $\mathrm{C}_{11} \mathrm{H}_{17} \mathrm{NO}_{2}$ : $194.1181[\mathrm{M}-\mathrm{H}]^{-}$, positive mode: found: 196.1337, 218.1158; calcd. for $\mathrm{C}_{11} \mathrm{H}_{17} \mathrm{NO}_{2}: 196.1338[\mathrm{M}+\mathrm{H}]^{+}, 218.1157[\mathrm{M}+\mathrm{Na}]^{+}$.

3,6-bis( $N, N$-Diethylamino)-2,7-dimethoxy-9-(3'-carboxyphenyl)xanthene (35): According<smiles>CCNc1cc2[o+]c3cc(N(CC)CC)c(OC)cc3c(-c3ccccc3C(=O)[O-])c2cc1OC</smiles>
to the GP1, phthalic anhydride $(0.15 \mathrm{~g}, 1.0 \mathrm{mmol}), p$-toluene sulfonic acid monohydrate (23 mg, $12 \mathrm{mmol})$ and compound $\mathbf{3 1}$ $(0.30 \mathrm{~g}, 1.5 \mathrm{mmol})$ was heated for $24 \mathrm{~h}$. After purification by chromatography on silica gel $(100 \mathrm{~g})$ with $\mathrm{CH}_{2} \mathrm{Cl}_{2} / \mathrm{MeOH}$ mixture $(10: 1 \rightarrow 4: 1)$, the title compound was isolated as violet foam $\left(70 \mathrm{mg}, 18 \%\right.$ ); decomp. $>300{ }^{\circ} \mathrm{C}$. HPLC: $50 / 50 \rightarrow 0 / 100(\mathrm{~A} / \mathrm{B})$ in $25 \mathrm{~min}, 1 \mathrm{~mL} / \mathrm{min}$, $25{ }^{\circ} \mathrm{C}, t_{\mathrm{R}}=3.8 \mathrm{~min}$, detection at $254 \mathrm{~nm} .{ }^{1} \mathrm{H}$ NMR (300 MHz, [D 6 ]DMSO): $\delta=1.02\left(\mathrm{t},{ }^{3} \mathrm{~J}=\right.$ $7.5,12 \mathrm{H}), 3.21$ (q, $\left.{ }^{3} J=7.5,8 \mathrm{H}\right), 3.46$ (s, $\left.6 \mathrm{H}, \mathrm{OMe}\right), 6.03$ (s, $\left.2 \mathrm{H}, 2 \times \mathrm{CH}, \mathrm{H}-4 / 5\right), 6.74$ (s, 2 H, $2 \times \mathrm{CH}, \mathrm{H}-1 / 8), 7.32$ (d, $\left.{ }^{3} J=7.8,1 \mathrm{H}, \mathrm{H}-7^{\prime}\right), 7.75$ (dt, $\left.{ }^{3} J=7.8,{ }^{4} J=1.8,1 \mathrm{H}, \mathrm{H}-5^{\prime}\right), 7.79$ (dt, 
$\left.{ }^{3} J=7.8,{ }^{4} J=1.8,1 \mathrm{H}, \mathrm{H}-6^{\prime}\right), 8.03\left(\mathrm{~d},{ }^{3} J=7.8,1 \mathrm{H}, \mathrm{H}-4^{\prime}\right) \mathrm{ppm} .{ }^{13} \mathrm{C}$ NMR $(75.6 \mathrm{MHz}$, [D 6 DMSO): $\delta=12.2\left(4 \times \underline{\mathrm{CH}}_{3} \mathrm{CH}_{2}\right), 44.8\left(4 \times \mathrm{CH}_{3} \underline{\mathrm{CH}}_{2}\right), 55.7\left(2 \times \mathrm{CH}_{3} \mathrm{O}\right), 106.2(2 \times \mathrm{CH}), 108.8$ (C), $109.0(\mathrm{C}), 109.1(2 \times \mathrm{CH}), 124.8(\mathrm{C}), 126.4(\mathrm{CH}), 130.0(2 \times \mathrm{CH}), 135.2(\mathrm{CH}), 142.7(\mathrm{C})$, 145.9 (C), 146.0 (C), $148.4(4 \times \mathrm{C}), 151.4$ (C), 168.4 (CO) ppm. ESI-MS, positive mode, $m / z$ (rel. int., \%): 503 (100) $[\mathrm{M}+\mathrm{H}]^{+}, 1027$ (6) [2M + Na $]^{+}$. HR-MS (ESI, positive mode): found: 503.2541, 525.2362; calcd. for $\mathrm{C}_{30} \mathrm{H}_{34} \mathrm{~N}_{2} \mathrm{O}_{5}: 503.2546[\mathrm{M}+\mathrm{H}]^{+}, 525.2365[\mathrm{M}+\mathrm{Na}]^{+}$.

3-(N,N-Diethylamino)-6-methoxy-9-(3'-carboxyphenyl)xanthene (39-Me): Compound 37<smiles>CCNc1ccc2c(-c3ccccc3C(=O)[O-])c3ccc(OC)cc3[o+]c2c1</smiles>
(1.6 g, $5.1 \mathrm{mmol})$, 3-methoxyphenol (38-Me) (0.74 g, $6.0 \mathrm{mmol})$ and $50 \%$ aq. sulphuric acid $(25 \mathrm{~mL})$ were placed into the flask equipped with a reflux condenser. The reaction mixture was heated at $125^{\circ} \mathrm{C}$ for $12 \mathrm{~h}$. After cooling to $0{ }^{\circ} \mathrm{C}$, the mixture was neutralized with $4.6 \mathrm{M}$ aq. $\mathrm{NaOH}(102 \mathrm{~mL})$. After the separation of the red oily product from the aqueous solution was complete (ca. $1 \mathrm{~h}$ ), the aqueous solution was decanted, and the red oil dissolved in $\mathrm{MeOH}$. The organic solution was dried and concentrated in vacuo. The residue was purified by chromatography on silica gel (200 g) with toluene / $\mathrm{MeOH}$ mixture $(2: 1)$ as an eluent, and the title compound was isolated as a red foam (0.96 g, 47\%); decomp. $>300{ }^{\circ} \mathrm{C}$. ${ }^{1} \mathrm{H}$ NMR (300 MHz, [D 6 ]DMSO): $\delta=1.10\left(\mathrm{t},{ }^{3} J=7.2,6\right.$ H), $3.36\left(\mathrm{q},{ }^{3} J=7.2,4 \mathrm{H}\right), 3.80$ (s, $\left.3 \mathrm{H}, \mathrm{OMe}\right), 6.46$ (m, $\left.3 \mathrm{H}, \mathrm{H}-5,7,8\right), 6.62\left(\mathrm{~d},{ }^{3} J=8.7,1 \mathrm{H}\right.$, $\mathrm{H}-1), 6.68\left(\mathrm{dd},{ }^{3} J=8.7,{ }^{4} \mathrm{~J}=2.4,1 \mathrm{H}, \mathrm{H}-2\right), 6.88\left(\mathrm{~d},{ }^{4} J=2.4,1 \mathrm{H}, \mathrm{H}-4\right), 7.26\left(\mathrm{~d},{ }^{3} J=7.8,1 \mathrm{H}\right.$, H-7'), $7.72\left(\mathrm{dt},{ }^{3} J=7.8,{ }^{4} J=1.8,1 \mathrm{H}, \mathrm{H}-5^{\prime}\right), 7.77\left(\mathrm{dt},{ }^{3} J=7.8,{ }^{4} J=1.8,1 \mathrm{H}, \mathrm{H}-6^{\prime}\right), 7.98\left(\mathrm{~d},{ }^{3} J\right.$ $\left.=7.8,1 \mathrm{H}, \mathrm{H}-4^{\prime}\right)$ ppm. ${ }^{13} \mathrm{C}$ NMR $\left(75.6 \mathrm{MHz},\left[\mathrm{D}_{6}\right] \mathrm{DMSO}\right): \delta=12.2\left(2 \times \underline{\mathrm{CH}}_{3} \mathrm{CH}_{2}\right), 43.7$ $\left(2 \times \mathrm{CH}_{3} \underline{\mathrm{CH}}_{2}\right), 55.5\left(\mathrm{CH}_{3} \mathrm{O}\right), 83.5(\mathrm{C}), 96.9(\mathrm{CH}), 100.8(\mathrm{CH}), 104.6(\mathrm{C}), 108.6(\mathrm{CH}), 111.3$ $(\mathrm{CH}), 111.4(\mathrm{C}), 123.9(\mathrm{CH}), 124.4(\mathrm{CH}), 126.3(\mathrm{C}), 128.5(\mathrm{CH}), 128.8(\mathrm{CH}), 129.9(\mathrm{CH})$, $135.4(\mathrm{CH}), 149.2$ (C), 152.13 (C), 152.17 (C), 152.3 (C), 160.9 (C), 168.7 (CO) ppm. ESIMS, positive mode, $m / z$ (rel. int., \%): $402(55)[\mathrm{M}+\mathrm{H}]^{+}, 424(100)[\mathrm{M}+\mathrm{Na}]^{+}, 825(32)[2 \mathrm{M}$ $+\mathrm{Na}]^{+}, 1226(41)[3 \mathrm{M}+\mathrm{Na}]^{+}$. HR-MS (ESI, positive mode): found: 402.1702, 424.1519; calcd. for $\mathrm{C}_{25} \mathrm{H}_{23} \mathrm{NO}_{4}: 402.1705[\mathrm{M}+\mathrm{H}]^{+}, 424.1525[\mathrm{M}+\mathrm{Na}]^{+}$.

Spiroamide 40-Me: Compound 39-Me $(0.20 \mathrm{~g}, 0.50 \mathrm{mmol})$ was suspended in dry 1,2added. The mixture was heated at reflux under Ar for $4 \mathrm{~h}$. All
volatile materials were evaporated in vacuo into a trap cooled
with dry ice, and the residue was flushed with argon. The 
resulted shiny red foam (compound 41-Me) was used in further operations without additional purification.

Dry acetonitrile $(10 \mathrm{~mL})$ was added to the flask, followed by aniline $(46 \mu \mathrm{L}, \mathrm{d}=1.02,0.50$ $\mathrm{mmol})$ and triethylamine $(0.21 \mathrm{~mL}, \mathrm{~d}=0.73,1.5 \mathrm{mmol})$. The reaction mixture was refluxed overnight, and the solvent was evaporated in vacuo. The residue was dissolved in $\mathrm{CHCl}_{3}(20$ $\mathrm{mL})$, and the organic solution was washed with water and saturated aqueous $\mathrm{NaHCO}_{3}(20 \mathrm{~mL}$ each) and dried. The residue was purified by chromatography on silica gel (50 g) with $\mathrm{CH}_{2} \mathrm{Cl}_{2}$ / MeOH mixture (20:1) as an eluent, and the title compound was isolated as a pink solid (210 mg, 88\%); decomp. $>300{ }^{\circ} \mathrm{C} .{ }^{1} \mathrm{H}$ NMR (300 MHz): $\delta=1.12\left(\mathrm{t},{ }^{3} J=7.5,6 \mathrm{H}\right), 3.28\left(\mathrm{q},{ }^{3} \mathrm{~J}=\right.$ 7.5, $4 \mathrm{H}$ ), 3.73 (s, $3 \mathrm{H}, \mathrm{OMe}), 6.22\left(\mathrm{~d},{ }^{3} J=9.0,1 \mathrm{H}, \mathrm{H}-8\right), 6.31\left(\mathrm{dd},{ }^{3} J=9.0,{ }^{4} J=2.4,1 \mathrm{H}, \mathrm{H}-\right.$ 7), 6.51 (m, 2 H, H-4,5), 6.59 (d, $\left.{ }^{3} J=8.7,1 \mathrm{H}, \mathrm{H}-1\right), 6.64$ (m, $\left.2 \mathrm{H}, \mathrm{H}-2^{\prime \prime}, 6^{\prime \prime}\right), 6.69$ (dd, ${ }^{3} J=$ 8.7, $\left.{ }^{4} J=2.0,1 \mathrm{H}, \mathrm{H}-2\right), 7.08$ (m, 4 H, H-7'+ H-3", $\left.4^{\prime \prime}, 5^{\prime \prime}\right), 7.49$ (m, $\left.2 \mathrm{H}, \mathrm{H}-5^{\prime} / 6^{\prime}\right), 7.98$ (m, $1 \mathrm{H}$, $\left.\mathrm{H}-4^{\prime}\right)$ ppm. ${ }^{13} \mathrm{C}$ NMR $(125.7 \mathrm{MHz}): \delta=12.4\left(2 \times \underline{\mathrm{CH}}_{3} \mathrm{CH}_{2}\right), 44.3\left(2 \times \mathrm{CH}_{3} \underline{\mathrm{CH}}_{2}\right), 55.3\left(\mathrm{CH}_{3} \mathrm{O}\right)$, $67.3(\mathrm{C}), 97.7(\mathrm{CH}), 100.6(\mathrm{CH}), 105.7(\mathrm{C}), 108.5(\mathrm{CH}), 110.9(\mathrm{CH}), 112.0(\mathrm{C}), 123.5(\mathrm{CH})$, $123.9(\mathrm{CH}), 127.0(\mathrm{CH}), 127.5(2 \times \mathrm{CH}), 128.5(\mathrm{CH}), 128.6(2 \times \mathrm{CH}), 128.8(\mathrm{CH}), 129.1(\mathrm{CH})$, 130.9 (C), 133.0 (CH), 136.0 (C), 148.9 (C), 152.7 (C), 152.8 (C), 152.9 (C), 160.3 (C), 167.7 (CO) ppm. ESI-MS, positive mode, $m / z$ (rel. int., \%): 499 (6) $[\mathrm{M}+\mathrm{Na}]^{+}, 975$ (100) $[2 \mathrm{M}+$ $\mathrm{Na}]^{+}, 1451$ (55) $[3 \mathrm{M}+\mathrm{Na}]^{+}$. HR-MS (ESI, positive mode): found: 477.2164; calcd. for $\mathrm{C}_{31} \mathrm{H}_{28} \mathrm{~N}_{2} \mathrm{O}_{3}: 477.2178[\mathrm{M}+\mathrm{H}]^{+}$.

Spiroamide 40-H: A solution of $\mathrm{BBr}_{3}(60 \mu \mathrm{L}, 0.63 \mathrm{mmol})$ in $\mathrm{CH}_{2} \mathrm{Cl}_{2}(5 \mathrm{~mL})$ was cooled to $\mathrm{Et}_{2} \mathrm{~N}$ in $\mathrm{CH}_{2} \mathrm{Cl}_{2}(5 \mathrm{~mL})$ was added dropwise. The mixture was stirred at $-20{ }^{\circ} \mathrm{C}$ for $30 \mathrm{~min}$, and then at the room temperature overnight. The reaction mixture was diluted with $\mathrm{CH}_{2} \mathrm{Cl}_{2}(10 \mathrm{~mL})$ and water $(5 \mathrm{~mL})$ was added carefully to it. The organic solution was washed with saturated aqueous $\mathrm{NaHCO}_{3}(20 \mathrm{~mL})$ and dried. The residue after evaporation of the solvent was purified by chromatography on silica gel (40 g) with $\mathrm{CH}_{2} \mathrm{Cl}_{2} / \mathrm{MeOH}$ mixture (20:1) as an eluent, and the title compound was isolated as a pink solid (60 mg, 62\%); decomp. $>290{ }^{\circ} \mathrm{C}$. ${ }^{1} \mathrm{H}$ NMR (300 MHz, [D 6 ]DMSO): $\delta=1.06\left(\mathrm{t},{ }^{3} J=7.5,6 \mathrm{H}\right), 3.32\left(\mathrm{q},{ }^{3} J=7.5,4 \mathrm{H}\right), 6.28\left(\mathrm{~d},{ }^{4} J=2.4,1 \mathrm{H}, \mathrm{H}-\right.$ 5), $6.41\left(\mathrm{dd},{ }^{3} J=9.0,{ }^{4} J=2.4,1 \mathrm{H}, \mathrm{H}-7\right), 6.47$ (m, $\left.2 \mathrm{H}, \mathrm{H}-1,2\right), 6.59$ (m, $\left.2 \mathrm{H}, \mathrm{H}-4,8\right), 6.75$ (m, 2 H, H-2", 6"), 7.13 (m, 4 H, H-7'+ H-3",4", 5'), 7.57 (m, 2 H, H-5'/6'), 7.88 (m, 1 H, H-4'), $9.78(\mathrm{~s}, 1 \mathrm{H}, \mathrm{OH}) \mathrm{ppm} .{ }^{13} \mathrm{C} \mathrm{NMR}\left(75.6 \mathrm{MHz},\left[\mathrm{D}_{6}\right] \mathrm{DMSO}\right): \delta=12.3\left(2 \times \underline{\mathrm{CH}}_{3} \mathrm{CH}_{2}\right), 43.5$ $\left(2 \times \mathrm{CH}_{3} \underline{\mathrm{CH}}_{2}\right), 66.2(\mathrm{C}), 97.1(\mathrm{CH}), 102.1(\mathrm{CH}), 105.2(\mathrm{C}), 108.4(\mathrm{CH}), 110.2(\mathrm{CH}), 112.1(\mathrm{C})$, 
$122.8(\mathrm{CH}), 123.7(\mathrm{CH}), 126.35(2 \times \mathrm{CH}), 126.4(\mathrm{CH}), 128.4(2 \times \mathrm{CH}), 128.5(\mathrm{CH}), 128.8(\mathrm{CH})$, $129.7(\mathrm{CH}), 133.3(\mathrm{CH}), 136.6(\mathrm{C}), 148.4(\mathrm{C}), 151.94(\mathrm{C}), 151.96(\mathrm{C}), 153.0(\mathrm{C}), 158.2(\mathrm{C})$, 166.5 (CO) ppm. ESI-MS, negative mode, m/z (rel. int., \%): 461 (100) [M - H] $]^{-}, 923$ (95) $[2 \mathrm{M}-\mathrm{H}]^{-}$, positive mode, $\mathrm{m} / \mathrm{z}$ (rel. int., \%): $485(10)[\mathrm{M}+\mathrm{Na}]^{+}, 947(100)[2 \mathrm{M}+\mathrm{Na}]^{+}, 1409$ (8) $[3 \mathrm{M}+\mathrm{Na}]^{+}$. HR-MS (ESI, negative mode): found: 461.1871, calcd. for $\mathrm{C}_{30} \mathrm{H}_{26} \mathrm{~N}_{2} \mathrm{O}_{3}$ : $461.1865[\mathrm{M}-\mathrm{H}]^{-}$.

Adduct 51: A solution of 4-nitro-2-aminophenol (49) $(3.1 \mathrm{~g}, 20 \mathrm{mmol})$ in dry DMF (30 mL)<smiles>O=[N+]([O-])c1ccc2c(c1)NCCOCCOCCOCCO2</smiles>
was prepared in the Schlenk-flask equipped with a septum and flushed with argon. Sodium carbonate $(4.2 \mathrm{~g}, 40 \mathrm{mmol})$ was added to the flask, the mixture was vigorously stirred, and then 1,11dichloro-3,6,9-trioxaundecane $\mathbf{5 0}(3.9 \mathrm{~mL}, \mathrm{~d}=1.18,20 \mathrm{mmol})$ was added. After heating at $90{ }^{\circ} \mathrm{C}$ for 12 hours, an additional portion of $\mathrm{Na}_{2} \mathrm{CO}_{3}(2.1 \mathrm{~g}, 20 \mathrm{mmol})$ was added, and after heating for $6 \mathrm{~h}$, another portion of $\mathrm{Na}_{2} \mathrm{CO}_{3}(2.1 \mathrm{~g}, 20 \mathrm{mmol})$ was added. After heating at $90{ }^{\circ} \mathrm{C}$ for $6 \mathrm{~h}$, the mixture was cooled. The solvent was evaporated in vacuo, and the residue was diluted with $\mathrm{CHCl}_{3}(200 \mathrm{~mL})$. The organic solution was washed with $1 \mathrm{M}$ aq. $\mathrm{NaOH}(3 \times 200 \mathrm{~mL})$, filtered through the cottonwool, and the solvent was removed in vacuo. The residue was purified by chromatography on $\mathrm{SiO}_{2}(300 \mathrm{~g})$ with $\mathrm{CH}_{2} \mathrm{Cl}_{2} / \mathrm{MeOH}$ mixture (100:1) as an eluent, and the title compound was isolated as a yellow solid (0.39 $\mathrm{g}$, $6.2 \%$ ). HPLC: $70 / 30 \rightarrow 0 / 100(\mathrm{~A} / \mathrm{B})$ in $25 \mathrm{~min}, 1 \mathrm{~mL} / \mathrm{min}, 25{ }^{\circ} \mathrm{C}, t_{\mathrm{R}}=12.8 \mathrm{~min}$, detection at 254 nm. ${ }^{1} \mathrm{H}$ NMR (300 MHz): $\delta=3.28$ (q, $\left.{ }^{3} J=5.4,2 \mathrm{H}\right), 3.66(\mathrm{~m}, 6 \mathrm{H}), 3.73(\mathrm{~m}, 2 \mathrm{H}), 3.78$ $(\mathrm{m}, 2 \mathrm{H}), 3.88(\mathrm{~m}, 2 \mathrm{H}), 4.17(\mathrm{~m}, 2 \mathrm{H}), 5.36(\mathrm{~m}, 1 \mathrm{H}, \mathrm{NH}), 6.68\left(\mathrm{~d},{ }^{3} J=9.0,1 \mathrm{H}\right), 7.34\left(\mathrm{~d},{ }^{4} J=\right.$ 2.7, $1 \mathrm{H}), 7.58\left(\mathrm{dd},{ }^{3} J=9.0,{ }^{4} J=2.7,1 \mathrm{H}\right) \mathrm{ppm} .{ }^{13} \mathrm{C}$ NMR $(75.6 \mathrm{MHz}): \delta=42.9\left(\mathrm{CH}_{2} \mathrm{~N}\right), 67.9$ $\left(\underline{\mathrm{CH}_{2} \mathrm{OAr}}\right), 68.3\left(\mathrm{CH}_{2}\right), 68.8\left(\mathrm{CH}_{2}\right), 69.8\left(\mathrm{CH}_{2}\right), 70.0\left(2 \times \mathrm{CH}_{2}\right), 70.4\left(\mathrm{CH}_{2}\right), 104.0(\mathrm{CH}), 109.0$ $(\mathrm{CH}), 113.2(\mathrm{CH}), 139.4(\mathrm{C}), 142.5(\mathrm{C}), 151.2$ (C) ppm. ESI-MS, positive mode, m/z (rel. int., $\%): 313(18)[\mathrm{M}+\mathrm{H}]^{+}, 335(100)[\mathrm{M}+\mathrm{Na}]^{+}$.

Compound 48a: According to the GP2, adduct $51(0.390 \mathrm{~g}, 1.3 \mathrm{mmol})$ was reduced in EtOAc<smiles>Nc1ccc2c(c1)NCCOCCOCCOCCO2</smiles>
and gave the title compound as a brown oil (0.35 g, 98\%). HPLC: $80 / 20 \rightarrow 50 / 50(\mathrm{~A} / \mathrm{B})$ in $25 \mathrm{~min}, 1 \mathrm{~mL} / \mathrm{min}, 25{ }^{\circ} \mathrm{C}, t_{\mathrm{R}}=2.8 \mathrm{~min}$, detection at $254 \mathrm{~nm} .{ }^{1} \mathrm{H}$ NMR (300 MHz): $\delta=3.18(\mathrm{~m}, 2 \mathrm{H}), 3.66$ $\left(\mathrm{m}, 11 \mathrm{H}, 4 \times \mathrm{CH}_{2}+\mathrm{NH}+\mathrm{NH}_{2}\right), 3.75\left(\mathrm{~m}, 4 \mathrm{H}, 2 \times \mathrm{CH}_{2}\right), 3.99(\mathrm{~m}, 2$ H), $5.94\left(\mathrm{dd},{ }^{3} J=8.1,{ }^{4} J=2.4,1 \mathrm{H}\right), 5.98\left(\mathrm{~d},{ }^{4} J=2.4,1 \mathrm{H}\right), 6.60\left(\mathrm{~d},{ }^{3} J=9.0,1 \mathrm{H}\right) \mathrm{ppm} .{ }^{13} \mathrm{C}$ NMR (75.6 MHz): $\delta=43.1\left(\mathrm{CH}_{2} \mathrm{~N}\right), 69.2\left(\mathrm{CH}_{2} \mathrm{OAr}\right), 69.7\left(\mathrm{CH}_{2}\right), 69.8\left(2 \times \mathrm{CH}_{2}\right), 69.9\left(\mathrm{CH}_{2}\right)$, 
$70.0\left(\mathrm{CH}_{2}\right), 70.4\left(\mathrm{CH}_{2}\right), 99.0(\mathrm{CH}), 102.3(\mathrm{CH}), 114.9(\mathrm{CH}), 139.7(\mathrm{C}), 140.8(\mathrm{C}), 141.6(\mathrm{C})$ ppm. ESI-MS, positive mode, m/z (rel. int., \%): $283(25)[\mathrm{M}+\mathrm{H}]^{+}, 305(100)[\mathrm{M}+\mathrm{Na}]^{+}$. HRMS (ESI, positive mode): found: 283.1652; calcd. for $\mathrm{C}_{14} \mathrm{H}_{22} \mathrm{~N}_{2} \mathrm{O}_{4}: 283.1658[\mathrm{M}+\mathrm{H}]^{+}$.

2-[2-[2-(2-Chloroethoxy)-ethoxy]-ethoxy]-ethyl acetate (54-Ac): A solution of alcohol 53 in the flask equipped with a reflux condenser, and acetic anhydride $(1.87 \mathrm{~mL}, \mathrm{~d}=1.08,20 \mathrm{mmol})$ with 3 drops of pyridine were added. The reaction mixture was refluxed for 26 hours. All volatile materials were evaporated in vacuo, and the residue was diluted with $\mathrm{CHCl}_{3}(50 \mathrm{~mL})$. The organic solution was washed with $5 \%$ aq. $\mathrm{NaHCO}_{3}(50 \mathrm{~mL}), 1 \mathrm{M}$ aq. $\mathrm{HCl}(2 \times 50 \mathrm{~mL})$, brine $(50 \mathrm{~mL})$ and dried. The solvent was removed in vacuo, the title compound was isolated as a colourless liquid $(4.10 \mathrm{~g}, 86 \%) .{ }^{1} \mathrm{H}$ NMR (300 MHz): $\delta=2.04$ (s, $3 \mathrm{H}), 3.66(\mathrm{~m}, 14 \mathrm{H}), 4.18(\mathrm{~m}, 2 \mathrm{H}) \mathrm{ppm} .{ }^{13} \mathrm{C}$ NMR $(75.5$ MHz): $\delta=20.9\left(\mathrm{CH}_{3}\right), 42.7\left(\mathrm{CH}_{2} \mathrm{Cl}\right), 63.6\left(\underline{\mathrm{CH}_{2} \mathrm{OAc}}\right), 69.1\left(\mathrm{CH}_{2}\right), 70.5\left(\mathrm{CH}_{2}\right), 70.57\left(\mathrm{CH}_{2}\right)$, $70.60\left(\mathrm{CH}_{2}\right), 70.62\left(\mathrm{CH}_{2}\right), 71.3\left(\mathrm{CH}_{2}\right), 171.0(\mathrm{CO}) \mathrm{ppm}$. CI-MS $\left(\mathrm{NH}_{3}\right)$, positive mode, m/z (rel. int., \%): $272(100)\left[\mathrm{M}+\mathrm{NH}_{4}\right]^{+}, 274$ (32) [M + $\left.\mathrm{NH}_{4}\right]^{+}$. HR-MS (ESI, positive mode): found: 277.0816; calcd. for $\mathrm{C}_{10} \mathrm{H}_{19} \mathrm{ClO}_{5}: 277.0819[\mathrm{M}+\mathrm{Na}]^{+}$.

2-[2-[2-(2-(4-Benzyloxy-2-nitrophenyloxy)ethoxy)-ethoxy]-ethoxy]-ethyl acetate (59): In a<smiles>CC(=O)OCCOCCOCCOCCOc1ccc(OCc2ccccc2)cc1[N+](=O)[O-]</smiles>
dry Schlenk-flask equipped with a septum and flushed with nitrogen, compound $\mathbf{5 8}$ $(2.8 \mathrm{~g}, 11 \mathrm{mmol})$ was dissolved in DMF $(30 \mathrm{~mL})$ and cooled to $0{ }^{\circ} \mathrm{C}$. Then $t$-BuOK $(1.3 \mathrm{~g}, 11 \mathrm{mmol})$ was added in small portions, and the mixture was stirred for $30 \mathrm{~min}$ at $0{ }^{\circ} \mathrm{C}$, until the clear red solution was obtained. After that, KI (0.19 g, $1.1 \mathrm{mmol})$ and compound 54-Ac (3.0 g, $12 \mathrm{mmol})$ were added to it, and the reaction mixture was heated at $90{ }^{\circ} \mathrm{C}$ for 3 days. All volatile materials were evaporated in vacuo, and the residue was diluted with $\mathrm{CHCl}_{3}(80 \mathrm{~mL})$. The organic solution was washed with saturated aq. $\mathrm{Na}_{2} \mathrm{CO}_{3}(2 \times 100 \mathrm{~mL})$, brine $(100 \mathrm{~mL})$ and dried. The solvent was removed in vacuo, the residue was purified by chromatography on silica gel (100 g) with $n$-hexane / EtOAc mixture $(4: 1 \rightarrow 0: 1)$ as an eluent, and the title compound was isolated as a yellow liquid (4.30 g, 82\%). HPLC: 50/50 $\rightarrow$ 0/100 (A/B) in $25 \mathrm{~min}, 1 \mathrm{~mL} / \mathrm{min}, 25^{\circ} \mathrm{C}, t_{\mathrm{R}}=10.3 \mathrm{~min}$, detection at $254 \mathrm{~nm} .{ }^{1} \mathrm{H}$ NMR $(300 \mathrm{MHz}): \delta=2.04(\mathrm{~s}, 3 \mathrm{H}), 3.70(\mathrm{~m}, 10 \mathrm{H}), 3.84(\mathrm{~m}, 2 \mathrm{H})$, $4.18(\mathrm{~m}, 4 \mathrm{H}), 5.03\left(\mathrm{~s}, 2 \mathrm{H}, \underline{\mathrm{CH}}_{2} \mathrm{Ph}\right), 7.04\left(\mathrm{~d},{ }^{3} J=9.0,1 \mathrm{H}\right), 7.12\left(\mathrm{dd},{ }^{3} J=9.0,{ }^{4} J=3.0,1 \mathrm{H}\right)$, $7.36\left(\mathrm{~m}, 5 \mathrm{H}, \mathrm{CH}_{2} \mathrm{Ph}\right), 7.42\left(\mathrm{~d},{ }^{4} J=3.0,1 \mathrm{H}\right) \mathrm{ppm} .{ }^{13} \mathrm{C}$ NMR $(75.5 \mathrm{MHz}): \delta=20.9\left(\mathrm{CH}_{3}\right)$, 
$63.6\left(\underline{\mathrm{CH}}_{2} \mathrm{OAc}\right), 69.1\left(\mathrm{CH}_{2}\right), 69.4\left(\mathrm{CH}_{2}\right), 70.48\left(\mathrm{CH}_{2}\right), 70.51\left(2 \times \mathrm{CH}_{2}\right), 70.6\left(\mathrm{CH}_{2}\right), 70.9$ $\left(\mathrm{CH}_{2}\right), 71.0\left(\underline{\mathrm{CH}_{2}} \mathrm{Ph}\right), 111.0(\mathrm{CH}), 117.2(\mathrm{CH}), 121.5(\mathrm{CH}), 127.5(2 \times \mathrm{CH}), 128.3(\mathrm{CH}), 128.7$ (2×CH), 135.9 (C), 140.1 (C), 146.7 (C), 152.2 (C), 171.0 (CO) ppm. ESI-MS, positive mode, m/z (rel. int., \%): 486 (100) [M + Na $]^{+}$. HR-MS (ESI, positive mode): found: 481.2180, 486.1735; calcd. for $\mathrm{C}_{23} \mathrm{H}_{29} \mathrm{NO}_{9}: 481.2186\left[\mathrm{M}+\mathrm{NH}_{4}\right]^{+}$, and $486.1740[\mathrm{M}+\mathrm{Na}]^{+}$.

\section{2-\{2-[2-(2-(4-Benzyloxy-2-aminophenyloxy)ethoxy)-ethoxy]-ethoxy\}-ethanol (60a): Into a}<smiles>Nc1cc(OCc2ccccc2)ccc1OCCOCCOCCOCCO</smiles>
dry three-necked flask equipped with a septum, dropping funnel and a reflux condenser with nitrogen inlet and outlet (on the top), compound 59 (4.7 g, $10 \mathrm{mmol}), \mathrm{MeOH}(50 \mathrm{~mL})$ and Raney-Ni (ca. $2.0 \mathrm{~g}$, slurry in $\mathrm{MeOH}$ ) were loaded under nitrogen. The reaction mixture was warmed-up to $60^{\circ} \mathrm{C}$ (bath), and a solution of $\mathrm{N}_{2} \mathrm{H}_{4} \cdot \mathrm{H}_{2} \mathrm{O}(2.0 \mathrm{~mL}, \mathrm{~d}=1.01,40.0 \mathrm{mmol})$ in $40 \mathrm{~mL}$ of $\mathrm{MeOH}$ was added dropwise with stirring at reflux during 1.5 hours. Then the reaction mixture was refluxed for 2 hours, cooled to room temperature and filtered through Celite ${ }^{\circledR}$. The filter-cake was washed with $\mathrm{MeOH}(3 \times 50 \mathrm{~mL})$, and the solvents were removed in vacuo. Then the residue was heated in high vacuo $(0.5$ torr $)$ at $60{ }^{\circ} \mathrm{C}$ for 1 hour. The product was isolated as a violet-brown oil which easily oxidized in $\operatorname{air}(3.78 \mathrm{~g}, 95 \%)$. ${ }^{1} \mathrm{H}$ NMR $(300 \mathrm{MHz}): \delta=3.58(\mathrm{~m}, 2 \mathrm{H}), 3.67$ $(\mathrm{m}, 10 \mathrm{H}), 3.76(\mathrm{~m}, 2 \mathrm{H}), 4.17(\mathrm{~m}, 2 \mathrm{H}), 4.95\left(\mathrm{~s}, 2 \mathrm{H}, \underline{\mathrm{CH}}_{2} \mathrm{Ph}\right), 6.25\left(\mathrm{dd},{ }^{3} J=8.7,{ }^{4} J=3.0,1\right.$ $\mathrm{H}), 6.38\left(\mathrm{~d},{ }^{4} J=3.0,1 \mathrm{H}\right), 6.72\left(\mathrm{~d},{ }^{3} J=8.7,1 \mathrm{H}\right), 7.35\left(\mathrm{~m}, 5 \mathrm{H}, \mathrm{CH}_{2} \underline{\mathrm{Ph}}\right) \mathrm{ppm} .{ }^{13} \mathrm{C}$ NMR $(75.5$ MHz): $\delta=61.7\left(\underline{\mathrm{CH}_{2}} \mathrm{OH}\right), 69.8\left(\mathrm{CH}_{2}\right), 69.9\left(\mathrm{CH}_{2}\right), 70.4\left(\mathrm{CH}_{2}\right), 70.58\left(\mathrm{CH}_{2}\right), 70.63\left(2 \times \mathrm{CH}_{2}\right)$, $72.6\left(\mathrm{CH}_{2}\right), 72.8\left(\underline{\mathrm{CH}}_{2} \mathrm{Ph}\right), 103.1(\mathrm{CH}), 103.5(\mathrm{CH}), 115.3(\mathrm{CH}), 127.4(2 \times \mathrm{CH}), 127.7(\mathrm{CH})$, $128.5(2 \times \mathrm{CH}), 137.6(\mathrm{C}), 138.8(\mathrm{C}), 141.0(\mathrm{C}), 154.5$ (C) ppm. ESI-MS, positive mode, m/z (rel. int., \%): 414 (100) [M + Na $]^{+}$. HR-MS (ESI, positive mode): found: 392.2068; calcd. for $\mathrm{C}_{21} \mathrm{H}_{29} \mathrm{NO}_{6}: 392.2073[\mathrm{M}+\mathrm{H}]^{+}$.

\section{2-[2-[2-(2-(4-Benzyloxy-2-aminophenyloxy)ethoxy)-ethoxy]-ethoxy]-ethyl tosylate (60b):}

$\mathrm{BnO}$<smiles>Nc1ccccc1OCCOCCOCCOCC[Se-]</smiles>

In the flask (No. 1) equipped with a reflux condenser, sodium $(0.15 \mathrm{mg}, 6.5 \mathrm{mmol})$ was dissolved in $i$-PrOH $(5 \mathrm{~mL})$ with heating at

$90{ }^{\circ} \mathrm{C}$. Then this solution was cooled to room temperature, and DMSO (5 mL) was added. In the dry flask (No. 2), compound $60 \mathrm{a}(0.50 \mathrm{mg}, 1.3 \mathrm{mmol})$ was added to the solution from the flask $1(2 \mathrm{~mL})$, the reaction mixture was stirred for $15 \mathrm{~min}$ at the room temperature, and tosyl chloride $(0.24 \mathrm{~g}, 1.3 \mathrm{mmol})$ was added in small portions. After stirring for 2 hours, the 
reaction mixture was diluted with $\mathrm{CHCl}_{3}(10 \mathrm{~mL})$. The organic solution was washed with water $(2 \times 20 \mathrm{~mL})$ and dried. The solvent was removed in vacuo, the residue was purified by chromatography on silica gel $(100 \mathrm{~g})$ with $n$-hexane / EtOAc mixture $(4: 1 \rightarrow 0: 1)$ as an eluent, and the title compound was isolated as a brown oil $(0.11 \mathrm{~g}, 16 \%)$. HPLC: 50/50 $\rightarrow$ $0 / 100(\mathrm{~A} / \mathrm{B})$ in $25 \mathrm{~min}, 1 \mathrm{~mL} / \mathrm{min}, 25{ }^{\circ} \mathrm{C}, t_{\mathrm{R}}=12.7 \mathrm{~min}$, detection at $254 \mathrm{~nm} .{ }^{1} \mathrm{H}$ NMR (300 MHz): $\delta=2.21(\mathrm{~s}, 3 \mathrm{H}), 3.65(\mathrm{~m}, 10 \mathrm{H}), 3.75(\mathrm{~m}, 2 \mathrm{H}), 4.06(\mathrm{~m}, 2 \mathrm{H}), 4.14(\mathrm{~m}, 2 \mathrm{H}), 4.94(\mathrm{~s}$, $\left.2 \mathrm{H}, \underline{\mathrm{CH}}_{2} \mathrm{Ph}\right), 6.28\left(\mathrm{dd},{ }^{3} J=8.7,{ }^{4} J=2.7,1 \mathrm{H}\right), 6.42\left(\mathrm{~d},{ }^{4} J=2.7,1 \mathrm{H}\right), 6.72\left(\mathrm{~d},{ }^{3} J=8.7,1 \mathrm{H}\right)$, $7.33\left(\mathrm{~m}, 7 \mathrm{H}, \mathrm{CH}_{2} \underline{\mathrm{Ph}}+\mathrm{CH}_{\mathrm{Ts}}+\mathrm{CH}_{\mathrm{Ts}}\right), 7.76\left(\mathrm{~d},{ }^{3} \mathrm{~J}=8.1,2 \mathrm{H}\right) \mathrm{ppm} .{ }^{13} \mathrm{C} \mathrm{NMR}(75.5 \mathrm{MHz}): \delta=$ $21.6\left(\mathrm{CH}_{3}\right), 68.7\left(\underline{\mathrm{CH}}_{2} \mathrm{OTs}\right), 69.2\left(\mathrm{CH}_{2}\right), 69.7\left(\mathrm{CH}_{2}\right), 69.8\left(\mathrm{CH}_{2}\right), 70.3\left(\mathrm{CH}_{2}\right), 70.46\left(\mathrm{CH}_{2}\right)$, $70.55\left(\mathrm{CH}_{2}\right), 70.58\left(\mathrm{CH}_{2}\right), 70.7\left(\underline{\mathrm{CH}_{2}} \mathrm{Ph}\right), 103.3(\mathrm{CH}), 104.0(\mathrm{CH}), 115.2(\mathrm{CH}), 127.5(2 \times \mathrm{CH})$, $127.8(\mathrm{CH}), 128.0(2 \times \mathrm{CH}), 128.5(2 \times \mathrm{CH}), 129.8(2 \times \mathrm{CH}), 132.9(\mathrm{C}), 137.4(\mathrm{C}), 137.7(\mathrm{C})$, 141.1 (C), 144.8 (C), 154.4 (C) ppm. ESI-MS, positive mode, m/z (rel. int., \%): 546 (100) [M $+\mathrm{H}]^{+}$.

\section{2-[2-[2-(2-(4-Benzyloxy-2-( $N$-tosylamino)phenyloxy)ethoxy)-ethoxy]-ethoxy]-ethyl}<smiles>[SH3+]Nc1cc(OCc2ccccc2)ccc1OCCOCCOCCOCC[Se-]</smiles>

tosylate (61): According to the GP3, compound 60a (3.9 g, $9.9 \mathrm{mmol})$ and tosyl chloride (3.8 g, $20 \mathrm{mmol})$ gave, after purification by chromatography on silica gel (400 g) with $\mathrm{CH}_{2} \mathrm{Cl}_{2} / \mathrm{MeOH}$ mixture (50:1) as an eluent, the title compound as a brown oil $(5.21 \mathrm{~g}, 75 \%) .{ }^{1} \mathrm{H}$ NMR $(300 \mathrm{MHz}): \delta=2.22$ (s, $3 \mathrm{H}), 2.41(\mathrm{~s}, 3 \mathrm{H}), 3.65(\mathrm{~m}, 12 \mathrm{H}), 3.84(\mathrm{~m}, 2 \mathrm{H}), 4.13(\mathrm{~m}, 2 \mathrm{H}), 4.98\left(\mathrm{~s}, 2 \mathrm{H}, \underline{\mathrm{CH}}_{2} \mathrm{Ph}\right), 6.55$ $\left(\mathrm{dd},{ }^{3} J=8.7,{ }^{4} J=2.7,1 \mathrm{H}\right), 6.72\left(\mathrm{~d},{ }^{4} J=2.7,1 \mathrm{H}\right), 7.12\left(\mathrm{~d},{ }^{3} J=8.1,2 \mathrm{H}_{\mathrm{TsNH}}\right), 7.24\left(\mathrm{~d},{ }^{3} J=\right.$ 8.7, $1 \mathrm{H}), 7.30\left(\mathrm{~d},{ }^{3} J=8.4,2 \mathrm{H}_{\mathrm{TsO}}\right), 7.36\left(\mathrm{~m}, 5 \mathrm{H}, \mathrm{CH}_{2} \underline{\mathrm{Ph}}\right), 7.59$ (d, $\left.{ }^{3} J=8.1,2 \mathrm{H}_{\mathrm{TsNH}}\right), 7.76$ (d, $\left.{ }^{3} J=8.4,2 \mathrm{H}_{\mathrm{TsO}}\right)$ ppm. ${ }^{13} \mathrm{C} \mathrm{NMR}(75.5 \mathrm{MHz}): \delta=21.5\left(\mathrm{CH}_{3}\right), 21.6\left(\mathrm{CH}_{3}\right), 68.7\left(\underline{\mathrm{CH}}_{2} \mathrm{OTs}\right)$, $69.2\left(\mathrm{CH}_{2}\right), 69.5\left(\mathrm{CH}_{2}\right), 70.5\left(\mathrm{CH}_{2}\right), 70.61\left(\mathrm{CH}_{2}\right), 70.64\left(\mathrm{CH}_{2}\right), 70.7\left(\mathrm{CH}_{2}\right), 70.8\left(\underline{\mathrm{CH}_{2}} \mathrm{Ph}\right)$, $71.0\left(\mathrm{CH}_{2}\right), 107.4(\mathrm{CH}), 111.3(\mathrm{CH}), 116.7(\mathrm{CH}), 127.3(2 \times \mathrm{CH}), 127.5(2 \times \mathrm{CH}), 127.90(\mathrm{CH})$, $127.94(2 \times \mathrm{CH}), 128.5(2 \times \mathrm{CH}), 129.4(2 \times \mathrm{CH}), 129.8(2 \times \mathrm{CH}), 136.5(\mathrm{C}), 137.0(\mathrm{C}), 142.7(\mathrm{C})$, 143.5 (C), 144.7 (C), 154.1 (C) ppm. ESI-MS, negative mode, m/z (rel. int., \%): 698 (100) [M $-\mathrm{H}]^{-}$, positive mode, $\mathrm{m} / \mathrm{z}$ (rel. int., \%): $722(100)[\mathrm{M}+\mathrm{Na}]^{+}$. HR-MS (ESI, positive mode): found: 717.2510, and 722.2066; calcd. for $\mathrm{C}_{35} \mathrm{H}_{41} \mathrm{NO}_{10} \mathrm{~S}_{2}$ : $717.2516\left[\mathrm{M}+\mathrm{NH}_{4}\right]^{+}$, and $722.2070[\mathrm{M}+\mathrm{Na}]^{+}$.

Crown ether 62: According to the GP4, compound 61 (6.4 g, $9.2 \mathrm{mmol})$ and $\mathrm{Cs}_{2} \mathrm{CO}_{3}(3.3 \mathrm{~g}$, $10 \mathrm{mmol})$ in DMF (40 $\mathrm{mL})$ gave, after purification by chromatography on silica gel (400 g) 
$\mathrm{BnO}$

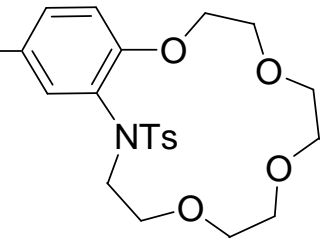

with $\mathrm{CH}_{2} \mathrm{Cl}_{2} / \mathrm{MeOH}$ mixture $(100: 1)$ as an eluent, the title compound as a light brown oil (2.61 g, 54\%). HPLC: 50/50 $\rightarrow$ $0 / 100(\mathrm{~A} / \mathrm{B})$ in $25 \mathrm{~min}, 1 \mathrm{~mL} / \mathrm{min}, 25{ }^{\circ} \mathrm{C}, t_{\mathrm{R}}=14.9 \mathrm{~min}$, detection at $254 \mathrm{~nm} .{ }^{1} \mathrm{H}$ NMR (300 MHz): $\delta=2.38(\mathrm{~s}, 3 \mathrm{H}), 3.63(\mathrm{~m}, 16 \mathrm{H})$, $4.94\left(\mathrm{~s}, 2 \mathrm{H}, \underline{\mathrm{CH}}_{2} \mathrm{Ph}\right), 6.63\left(\mathrm{~d},{ }^{3} J=8.7,1 \mathrm{H}\right), 6.86(\mathrm{~m}, 2 \mathrm{H}), 7.20\left(\mathrm{~d},{ }^{3} J=8.1,2 \mathrm{H}_{\mathrm{TsN}}\right), 7.35$ (m, $\left.5 \mathrm{H}, \mathrm{CH}_{2} \underline{\mathrm{Ph}}\right), 7.59\left(\mathrm{~d},{ }^{3} J=8.1,2 \mathrm{H}_{\mathrm{TsNH}}\right) \mathrm{ppm} .{ }^{13} \mathrm{C} \mathrm{NMR}(75.5 \mathrm{MHz}): \delta=21.5\left(\mathrm{CH}_{3}\right), 50.3$ $\left(\mathrm{CH}_{2}\right), 66.8\left(\mathrm{CH}_{2}\right), 68.9\left(\mathrm{CH}_{2}\right), 69.8\left(\mathrm{CH}_{2}\right), 70.1\left(\mathrm{CH}_{2}\right), 70.6\left(\mathrm{CH}_{2}\right), 70.7\left(\mathrm{CH}_{2}\right), 70.8$ $\left(\underline{\mathrm{CH}}_{2} \mathrm{Ph}\right), 71.3\left(\mathrm{CH}_{2}\right), 112.3(\mathrm{CH}), 116.0(\mathrm{CH}), 119.1(\mathrm{CH}), 127.6(2 \times \mathrm{CH}), 127.91(2 \times \mathrm{CH})$, $127.96(\mathrm{CH}), 128.2(\mathrm{C}), 128.5(2 \times \mathrm{CH}), 128.9(2 \times \mathrm{CH}), 136.8(\mathrm{C}), 137.1(\mathrm{C}), 142.7(\mathrm{C}), 150.0$ (C), 152.1 (C) ppm. ESI-MS, negative mode, m/z (rel. int., \%): 544 (45) [M + OH] $]^{-}, 562$ (80) $[\mathrm{M}+\mathrm{Cl}]^{-}, 1089(100)[2 \mathrm{M}+\mathrm{Cl}]^{-}$, positive mode, $\mathrm{m} / \mathrm{z}$ (rel. int., \%): $550(100)[\mathrm{M}+\mathrm{Na}]^{+}$. HRMS (ESI, positive mode): found: 528.2049, 545.2313, 550.1869, and 566.1608; calcd. for $\mathrm{C}_{28} \mathrm{H}_{33} \mathrm{NO}_{7} \mathrm{~S}: 528.2056[\mathrm{M}+\mathrm{H}]^{+}, 545.2321\left[\mathrm{M}+\mathrm{NH}_{4}\right]^{+}, 550.1875[\mathrm{M}+\mathrm{Na}]^{+}$, and 566.1615 $[\mathrm{M}+\mathrm{K}]^{+}$.

Crown ether 63a: Method 1: According to the GP4, compound 60b $(0.10 \mathrm{~g}, 0.18 \mathrm{mmol})$ and

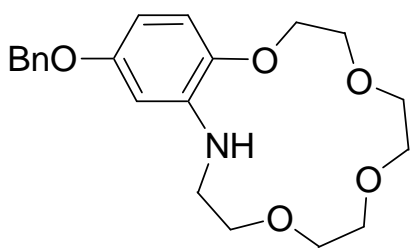
caesium carbonate $(63 \mathrm{mg}, 0.19 \mathrm{mmol})$ in DMF $(2 \mathrm{~mL})$ gave, after purification by chromatography on silica gel $(25 \mathrm{~g})$ with $\mathrm{CH}_{2} \mathrm{Cl}_{2} /$ $\mathrm{MeOH}$ mixture (100:1) as an eluent, the title compound as a slightly brown oil (20 mg, 29\%).

Method 2: In a dry flask (No. 1) equipped with a septum, sodium (1.5 g, $65 \mathrm{mmol}$ ) was dissolved in the solution of naphthalene $(11 \mathrm{~g}, 86 \mathrm{mmol})$ in DME $(50 \mathrm{~mL})$ with stirring for 2 $\mathrm{h}$ at room temperature. In a dry flask (No. 2) equipped with a septum, to a solution of compound $62(0.94 \mathrm{~g}, 1.8 \mathrm{mmol})$ in DME $(20 \mathrm{~mL})$, the dark green solution (from the flask 1) was added dropwise at $-78{ }^{\circ} \mathrm{C}$, until the stable green color was persisted. Then the saturated solution of $\mathrm{NaHCO}_{3}(10 \mathrm{~mL})$ was added to the reaction mixture, and it was warmed-up to room temperature. Dry $\mathrm{K}_{2} \mathrm{CO}_{3}(7.0 \mathrm{~g})$ was added, and the mixture was stirred overnight. Then it was filtered, the filter-cake was washed with diethyl ether $(3 \times 40 \mathrm{~mL})$, and the solvents were removed in vacuo. The residue was purified by chromatography on silica gel (100 g) with $n$ hexane / EtOAc mixture (1:1) as an eluent, and the title compound was isolated as slightly brown oil (410 mg, 62\%). HPLC: $50 / 50 \rightarrow 0 / 100(\mathrm{~A} / \mathrm{B})$ in $25 \mathrm{~min}, 1 \mathrm{~mL} / \mathrm{min}, 25^{\circ} \mathrm{C}, t_{\mathrm{R}}=6.8$ min, detection at $254 \mathrm{~nm} .{ }^{1} \mathrm{H}$ NMR (300 MHz): $\delta=3.20(\mathrm{~m}, 2 \mathrm{H}), 3.66(\mathrm{~m}, 8 \mathrm{H}), 3.77(\mathrm{~m}, 4$ $\mathrm{H}), 4.14(\mathrm{~m}, 2 \mathrm{H}), 4.96\left(\mathrm{~s}, 2 \mathrm{H}, \underline{\mathrm{CH}}_{2} \mathrm{Ph}\right), 5.16$ (br s, $\left.1 \mathrm{H}\right), 6.16\left(\mathrm{dd},{ }^{3} \mathrm{~J}=8.7,{ }^{4} \mathrm{~J}=2.7,1 \mathrm{H}\right)$, $6.25\left(\mathrm{~d},{ }^{4} J=2.7,1 \mathrm{H}\right), 6.67\left(\mathrm{~d},{ }^{3} J=8.7,1 \mathrm{H}\right), 7.35\left(\mathrm{~m}, 5 \mathrm{H}, \mathrm{CH}_{2} \underline{\mathrm{Ph}}\right) \mathrm{ppm} .{ }^{13} \mathrm{C}$ NMR $(75.5$ 
MHz): $\delta=43.1\left(\mathrm{CH}_{2} \mathrm{~N}\right), 69.0\left(\mathrm{CH}_{2}\right), 69.5\left(\mathrm{CH}_{2}\right), 69.7\left(\mathrm{CH}_{2}\right), 69.8\left(\mathrm{CH}_{2}\right), 69.9\left(\mathrm{CH}_{2}\right), 70.0$ $\left(\mathrm{CH}_{2}\right), 70.3\left(\mathrm{CH}_{2}\right), 70.4\left(\underline{\mathrm{CH}}_{2} \mathrm{Ph}\right), 99.2(\mathrm{CH}), 100.0(\mathrm{CH}), 113.5(\mathrm{CH}), 127.6(2 \times \mathrm{CH}), 127.7$ (CH), $128.5(2 \times \mathrm{CH}), 137.5(\mathrm{C}), 140.8$ (C), 141.1 (C), 154.7 (C) ppm. ESI-MS, positive mode, m/z (rel. int., \%): $396(100)[\mathrm{M}+\mathrm{Na}]^{+}, 768(24)[2 \mathrm{M}+\mathrm{Na}]^{+}$. HR-MS (ESI, positive mode): found: 374.1961 , and 396.1781; calcd. for $\mathrm{C}_{21} \mathrm{H}_{27} \mathrm{NO}_{5}$ : $374.1967[\mathrm{M}+\mathrm{H}]^{+}$, and $396.1787[\mathrm{M}$ $+\mathrm{Na}]^{+}$.

Acetanilide 63b: In a flask equipped with a reflux condenser, compound 63a $(0.12 \mathrm{mg}, 0.32$<smiles>O=C(c1ccccc1)N1CCOCCOCCOCCOc2ccc(Br)cc21</smiles>
mmol) was dissolved in $\mathrm{AcOH}(7 \mathrm{~mL})$. To this solution, acetic anhydride $(45.3 \mu \mathrm{L}, \mathrm{d}=1.08,0.48 \mathrm{mmol})$ was added dropwise. The reaction mixture was refluxed overnight, cooled to room temperature, and poured into the cold water. The aqueous suspension was extracted with $\mathrm{CH}_{2} \mathrm{Cl}_{2}(3 \times 10 \mathrm{~mL})$. All volatile materials were evaporated in vacuo. The obtained oil was diluted with $\mathrm{CH}_{2} \mathrm{Cl}_{2}$ and the solvent was evaporated once more. The latter operation was repeated two times. The title compound was isolated as a very light brown oil (132 mg, 99\%). ${ }^{1} \mathrm{H}$ NMR (300 MHz): $\delta=1.75$ (s, $\left.3 \mathrm{H}, \mathrm{CH}_{3}\right), 3.14$ (m, $\left.1 \mathrm{H}\right), 3.62$ $(\mathrm{m}, 8 \mathrm{H}), 3.78(\mathrm{~m}, 4 \mathrm{H}), 4.02(\mathrm{~m}, 1 \mathrm{H}), 4.16(\mathrm{~m}, 1 \mathrm{H}), 4.22(\mathrm{~m}, 1 \mathrm{H}), 4.98\left(\mathrm{~s}, 2 \mathrm{H}, \underline{\mathrm{CH}}_{2} \mathrm{Ph}\right)$, $6.75\left(\mathrm{~d},{ }^{4} J=3.0,1 \mathrm{H}\right), 6.80\left(\mathrm{~d},{ }^{3} J=9.0,1 \mathrm{H}\right), 6.87\left(\mathrm{dd},{ }^{3} J=9.0,{ }^{4} J=3.0,1 \mathrm{H}\right), 7.35(\mathrm{~m}, 5 \mathrm{H}$, $\left.\mathrm{CH}_{2} \underline{\mathrm{Ph}}\right)$ ppm. ${ }^{13} \mathrm{C}$ NMR $(75.5 \mathrm{MHz}): \delta=21.9\left(\mathrm{CH}_{3}\right), 49.1\left(\mathrm{CH}_{2} \mathrm{~N}\right), 67.6\left(\mathrm{CH}_{2}\right), 68.0\left(\mathrm{CH}_{2}\right)$, $69.1\left(\mathrm{CH}_{2}\right), 70.07\left(\mathrm{CH}_{2}\right), 70.14\left(\mathrm{CH}_{2}\right), 70.19\left(\mathrm{CH}_{2}\right), 70.8\left(\underline{\mathrm{CH}_{2}} \mathrm{Ph}\right), 71.7\left(\mathrm{CH}_{2}\right), 112.7(\mathrm{CH})$, $115.1(\mathrm{CH}), 116.4(\mathrm{CH}), 127.5(2 \times \mathrm{CH}), 128.1(\mathrm{CH}), 128.6(2 \times \mathrm{CH}), 132.5(\mathrm{C}), 136.6(\mathrm{C})$, 149.1 (C), 152.7 (C), 171.3 (CO) ppm. ESI-MS, positive mode, m/z (rel. int., \%): 438 (100) $[\mathrm{M}+\mathrm{Na}]^{+}, 853(100)[2 \mathrm{M}+\mathrm{Na}]^{+}$. HR-MS (ESI, positive mode): found: 438.1887; calcd. for $\mathrm{C}_{23} \mathrm{H}_{29} \mathrm{NO}_{6}: 438.1893[\mathrm{M}+\mathrm{Na}]^{+}$.

$N$-ethyl crown ether 63c: A solution of $\mathrm{BH}_{3}$ in THF $(1 \mathrm{M} ; 0.5 \mathrm{~mL})$ at $0{ }^{\circ} \mathrm{C}$, was added to<smiles>CCN1CCOCCOCCOCCOc2ccc(Br)cc21</smiles>
compound $63 \mathbf{b}(0.13 \mathrm{mg}, 0.32 \mathrm{mmol})$ in dry THF $(3 \mathrm{~mL})$, and the mixture was heated at reflux overnight. Then it was cooled to $0{ }^{\circ} \mathrm{C}$, an excess of $\mathrm{BH}_{3}$ was carefully destroyed by adding $\mathrm{MeOH}(1 \mathrm{~mL})$, and water $(1 \mathrm{~mL})$ was added. After stirring at room temperature for $20 \mathrm{~min}$, the mixture was diluted with $\mathrm{Et}_{2} \mathrm{O}(20 \mathrm{~mL})$ and the organic layer was separated. The aqueous layer was extracted with $\mathrm{Et}_{2} \mathrm{O}(3 \times 10 \mathrm{~mL})$, then the combined organic layers were washed with saturated aqueous $\mathrm{NaHCO}_{3}(40 \mathrm{~mL})$ and brine $(40 \mathrm{~mL})$. The organic solution was filtered through a piece of cottonwool, and the solvent was removed in vacuo. The title 
compound was isolated as a light brown oil $(0.127 \mathrm{mg}, 99 \%) .{ }^{1} \mathrm{H}$ NMR (300 MHz): $\delta=1.06$ $\left(\mathrm{t},{ }^{3} J=7.2,3 \mathrm{H}\right), 3.18\left(\mathrm{q},{ }^{3} J=7.2,2 \mathrm{H}\right), 3.26(\mathrm{~m}, 2 \mathrm{H}), 3.66(\mathrm{~m}, 8 \mathrm{H}), 3.75(\mathrm{~m}, 2 \mathrm{H}), 3.88$ (m, $2 \mathrm{H}), 4.09$ (m, $2 \mathrm{H}), 4.97$ (s, $\left.2 \mathrm{H}, \underline{\mathrm{CH}_{2}} \mathrm{Ph}\right), 6.44\left(\mathrm{dd},{ }^{3} J=8.7,{ }^{4} \mathrm{~J}=2.7,1 \mathrm{H}\right), 6.56\left(\mathrm{~d},{ }^{4} J=2.7,1\right.$ $\mathrm{H}), 6.69\left(\mathrm{~d},{ }^{3} \mathrm{~J}=8.7,1 \mathrm{H}\right), 7.36\left(\mathrm{~m}, 5 \mathrm{H}, \mathrm{CH}_{2} \underline{\mathrm{Ph}}\right) \mathrm{ppm} .{ }^{13} \mathrm{C} \mathrm{NMR}(125.7 \mathrm{MHz}): \delta=12.7$ $\left(\underline{\mathrm{CH}}_{3} \mathrm{CH}_{2}\right), 46.3\left(\mathrm{CH}_{3} \underline{\mathrm{CH}}_{2}\right), 53.0\left(\mathrm{CH}_{2} \mathrm{~N}\right), 68.5\left(\mathrm{CH}_{2}\right), 69.0\left(\underline{\mathrm{CH}_{2}}\right), 69.97\left(\mathrm{CH}_{2}\right), 69.99\left(\mathrm{CH}_{2}\right)$, $70.4\left(\mathrm{CH}_{2}\right), 70.5\left(2 \times \mathrm{CH}_{2}\right), 70.6\left(\mathrm{CH}_{2}\right), 105.4(\mathrm{CH}), 108.2(\mathrm{CH}), 113.3(\mathrm{CH}), 127.5(2 \times \mathrm{CH})$, $127.8(\mathrm{CH}), 128.4(2 \times \mathrm{CH}), 137.3(\mathrm{C}), 141.4(\mathrm{C}), 146.7$ (C), $153.1(\mathrm{C})$, ppm. ESI-MS, positive mode, m/z (rel. int., \%): 424 (100) $[\mathrm{M}+\mathrm{Na}]^{+}$. HR-MS (ESI, positive mode): found: 402.2280; calcd. for $\mathrm{C}_{23} \mathrm{H}_{31} \mathrm{NO}_{5}: 402.2280[\mathrm{M}+\mathrm{H}]^{+}$.

Crown ether 63d: To a solution of crown ether 63a $(0.12 \mathrm{mg}, 0.32 \mathrm{mmol})$ in dry $\mathrm{MeCN}(2$

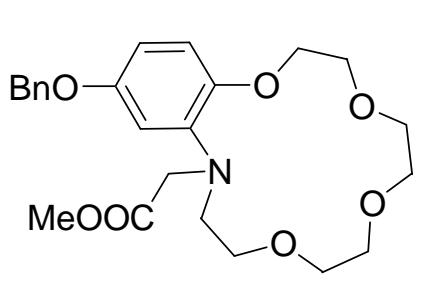
$\mathrm{mL})$ in a dry Schlenk-flask equipped with a septum and flushed with argon, DIPEA (62 $\mu \mathrm{L}, \mathrm{d}=0.76,0.36 \mathrm{mmol})$ and methyl 2bromoacetate $(32 \mu \mathrm{L}, \mathrm{d}=1.62,0.34 \mathrm{mmol})$ were added at room temperature. The reaction mixture was heated at $100{ }^{\circ} \mathrm{C}$ (bath temperature) for 1 day. All volatile materials were evaporated in vacuo. The residue was purified by chromatography on silica gel (20 g) with $\mathrm{CH}_{2} \mathrm{Cl}_{2} / \mathrm{MeOH}$ mixture (30:1 $\rightarrow 10: 1)$ as an eluent, and the title compound was isolated as slightly brown oil (0.11 g, 73\%). ${ }^{1} \mathrm{H}$ NMR (300 MHz): $\delta=3.46$ (m, $\left.2 \mathrm{H}\right), 3.59(\mathrm{~m}, 2 \mathrm{H}), 3.68\left(\mathrm{~m}, 11 \mathrm{H}, 4 \times \mathrm{CH}_{2}\right.$ $\left.+\mathrm{CH}_{3}\right), 3.85(\mathrm{~m}, 2 \mathrm{H}), 4.07(\mathrm{~m}, 2 \mathrm{H}), 4.15\left(\mathrm{~s}, 2 \mathrm{H}, \mathrm{CH}_{2}\right), 4.95\left(\mathrm{~s}, 2 \mathrm{H}, \underline{\mathrm{CH}}_{2} \mathrm{Ph}\right), 6.46\left(\mathrm{dd},{ }^{3} J=\right.$ $\left.8.7,{ }^{4} J=2.7,1 \mathrm{H}\right), 6.59\left(\mathrm{~d},{ }^{4} J=2.7,1 \mathrm{H}\right), 6.72\left(\mathrm{~d},{ }^{3} J=8.7,1 \mathrm{H}\right), 7.35\left(\mathrm{~m}, 5 \mathrm{H}, \mathrm{CH}_{2} \underline{\mathrm{Ph}}\right) \mathrm{ppm}$. ${ }^{13} \mathrm{C}$ NMR $(125.7 \mathrm{MHz}): \delta=51.5\left(\mathrm{CH}_{2} \mathrm{COOMe}\right), 52.0\left(\underline{\mathrm{CH}}_{2} \mathrm{COOMe}\right), 53.4\left(\mathrm{CH}_{2} \mathrm{~N}\right), 69.1$ $\left(\mathrm{CH}_{2}\right), 69.6\left(\mathrm{CH}_{2}\right), 70.0\left(\mathrm{CH}_{2}\right), 70.2\left(\mathrm{CH}_{2}\right), 70.5\left(\mathrm{CH}_{2}\right), 70.7\left(\mathrm{CH}_{2}\right), 70.8\left(\underline{\mathrm{CH}}_{2} \mathrm{Ph}\right), 70.9$ $\left(\mathrm{CH}_{2}\right), 106.5(\mathrm{CH}), 108.6(\mathrm{CH}), 114.9(\mathrm{CH}), 127.5(2 \times \mathrm{CH}), 127.8(\mathrm{CH}), 128.4(2 \times \mathrm{CH}), 137.3$ (C), 141.0 (C), 146.1 (C), 153.4 (C), $172.1\left(\mathrm{CH}_{2}\right.$ COOMe) ppm. ESI-MS, positive mode, m/z (rel. int., \%): 468 (100) [M + Na] ${ }^{+}$. HR-MS (ESI, positive mode): found: 446.2176; calcd. for $\mathrm{C}_{24} \mathrm{H}_{31} \mathrm{NO}_{7}: 446.2179[\mathrm{M}+\mathrm{H}]^{+}$.

Phenol 42a: According to the GP5, compound 63a (60 mg, $0.16 \mathrm{mmol})$ with $10 \% \mathrm{Pd} / \mathrm{C}(12$

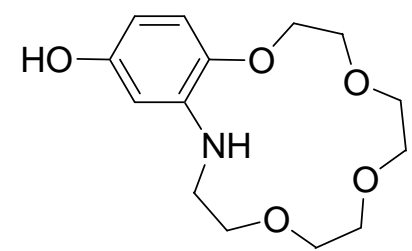
$\mathrm{mg})$ in $\mathrm{AcOH}(3 \mathrm{~mL})$ gave the title compound as a brown oil which solidified at $-10{ }^{\circ} \mathrm{C}$ in ca. one week; yield (44 mg, 98\%). ${ }^{1} \mathrm{H}$ NMR (300 MHz): $\delta=3.14$ (m, $2 \mathrm{H}), 3.68(\mathrm{~m}, 10 \mathrm{H}), 3.77$ (m, $2 \mathrm{H}), 3.99$ $(\mathrm{m}, 2 \mathrm{H}), 5.30$ (br s, $2 \mathrm{H}, \mathrm{OH}+\mathrm{NH}), 6.04(\mathrm{~m}, 1 \mathrm{H}), 6.10(\mathrm{~m}, 1 \mathrm{H})$, $6.58\left(\mathrm{~d},{ }^{3} J=8.7,1 \mathrm{H}\right) \mathrm{ppm} .{ }^{13} \mathrm{C} \mathrm{NMR}(125.7 \mathrm{MHz}): \delta=43.3\left(\mathrm{CH}_{2} \mathrm{~N}\right), 69.0\left(\mathrm{CH}_{2}\right), 69.6\left(\mathrm{CH}_{2}\right)$, 
$69.7\left(2 \times \mathrm{CH}_{2}\right), 69.8\left(\mathrm{CH}_{2}\right), 70.0\left(\mathrm{CH}_{2}\right), 70.3\left(\mathrm{CH}_{2}\right), 99.0(\mathrm{CH}), 101.8(\mathrm{CH}), 114.1(\mathrm{CH}), 140.4$ (C), 140.7 (C), 151.4 (C) ppm. ESI-MS, negative mode, m/z (rel. int., \%): 282 (100) [M - H] , $565(100)[2 \mathrm{M}-\mathrm{H}]^{-}$, positive mode, $\mathrm{m} / \mathrm{z}$ (rel. int., \%): $306(100)[\mathrm{M}+\mathrm{Na}]^{+}, 589(60)[2 \mathrm{M}+$ $\mathrm{Na}]^{+}$. HR-MS (ESI, negative mode): found: 282.1347; calcd. for $\mathrm{C}_{14} \mathrm{H}_{21} \mathrm{NO}_{5}$ : 282.1341 [M $\mathrm{H}]^{-}$.

Phenol 42c: According to the GP5, compound 63d $(72 \mathrm{mg}, 0.16 \mathrm{mmol})$ with $10 \% \mathrm{Pd} / \mathrm{C}(15$<smiles>CC(=O)CN1CCOCCOCCOCCOc2ccc(O)cc21</smiles>
$\mathrm{mg})$ in $\mathrm{MeOH}(3 \mathrm{~mL})$ gave the title compound as a brownish oil, yield (56 mg, 99\%). ${ }^{1} \mathrm{H}$ NMR (300 MHz): $\delta=3.45(\mathrm{~m}, 2 \mathrm{H}), 3.58$ $(\mathrm{m}, 2 \mathrm{H}), 3.68\left(\mathrm{~m}, 11 \mathrm{H}, 4 \times \mathrm{CH}_{2}+\mathrm{CH}_{3}\right), 3.84(\mathrm{~m}, 2 \mathrm{H}), 4.06(\mathrm{~m}, 2$ H), 4.15 (s, $2 \mathrm{H}), 6.35(\mathrm{~m}, 1 \mathrm{H}), 6.52(\mathrm{~m}, 1 \mathrm{H}), 6.64\left(\mathrm{~d},{ }^{3} J=8.7,1 \mathrm{H}\right)$ ppm. ${ }^{13} \mathrm{C}$ NMR (75.5 MHz): $\delta=51.5\left(\mathrm{CH}_{2} \mathrm{COOMe}\right), 52.4$ $\left(\underline{\mathrm{CH}}_{2} \mathrm{COOMe}\right), 53.7\left(\mathrm{CH}_{2} \mathrm{~N}\right), 67.9\left(\mathrm{CH}_{2}\right), 68.9\left(\mathrm{CH}_{2}\right), 69.5\left(\mathrm{CH}_{2}\right), 69.9\left(\mathrm{CH}_{2}\right), 70.2\left(\mathrm{CH}_{2}\right)$, $70.6\left(\mathrm{CH}_{2}\right), 70.7\left(\mathrm{CH}_{2}\right), 108.7(2 \times \mathrm{CH}), 115.5(\mathrm{CH}), 140.7(\mathrm{CH}), 145.7(\mathrm{C}), 150.6(\mathrm{C}), 172.2$ $\left(\mathrm{CH}_{2} \text { COOMe) ppm. ESI-MS, negative mode, m/z (rel. int., \%): } 354 \text { (45) [M - H] }\right]^{-}, 195$ (100), positive mode, m/z (rel. int., \%): $356(20)[\mathrm{M}+\mathrm{H}]^{+} .378(100)[\mathrm{M}+\mathrm{Na}]^{+}$. HR-MS (ESI, positive mode): found: 378.1529 ; calcd. for $\mathrm{C}_{17} \mathrm{H}_{25} \mathrm{NO}_{7}$ : $378.1523[\mathrm{M}+\mathrm{Na}]^{+}$.

Phenol 68: Methyl ether 67 (0.18 g, $0.55 \mathrm{mmol})$ was suspended in dry 2,4,6-collidine ( $1 \mathrm{~mL})$,<smiles>Oc1cccc(N2CCOCCOCCOCCOCC2)c1</smiles>
then LiI $(0.29 \mathrm{~g}, 2.2 \mathrm{mmol})$ was added and the mixture was heated overnight in a closed thick-wall glass tube (bath temp. $180^{\circ} \mathrm{C}$ ). After cooling, the reaction mixture was transferred into the concentrated hydrochloric acid $(2 \mathrm{~mL})$. The resulted solution was cooled to $0{ }^{\circ} \mathrm{C}$ and neutralized with saturated aq. $\mathrm{NaHCO}_{3}$. Then it was extracted with $\mathrm{CH}_{2} \mathrm{Cl}_{2}(3 \times 10 \mathrm{~mL})$. The organic solution was filtered through a piece of cottonwool, and the solvent was removed in vacuo. The residue was purified by chromatography on silica gel $(20 \mathrm{~g})$ with $n$-hexane / EtOAc mixture $(1: 2 \rightarrow 1: 4)$ as an eluent, and the title compound was isolated as a brownish oil (77 mg, 45\%). ${ }^{1} \mathrm{H}$ NMR (300 MHz): $\delta=3.53(\mathrm{~m}, 4 \mathrm{H}), 3.63(\mathrm{~m}, 12$ $\mathrm{H}), 3.72(\mathrm{~m}, 4 \mathrm{H}), 6.13(\mathrm{~m}, 2 \mathrm{H}), 6.20(\mathrm{~m}, 1 \mathrm{H}), 7.02$ (m, $1 \mathrm{H}) \mathrm{ppm} .{ }^{13} \mathrm{C}$ NMR $(125.7 \mathrm{MHz}): \delta$ $=52.6\left(2 \times \mathrm{CH}_{2} \mathrm{~N}\right), 68.6\left(2 \times \mathrm{CH}_{2}\right), 70.2\left(4 \times \mathrm{CH}_{2}\right), 71.3\left(2 \times \mathrm{CH}_{2}\right), 98.6(\mathrm{CH}), 102.9(\mathrm{CH}), 104.1$ (CH), $130.0(\mathrm{CH}), 149.1(\mathrm{C}), 156.9$ (C) ppm. ESI-MS, negative mode, m/z (rel. int., \%): 310 (100) $[\mathrm{M}-\mathrm{H}]^{-}, 621(15)[2 \mathrm{M}-\mathrm{H}]^{-}$, positive mode, m/z (rel. int., \%): $334(100)[\mathrm{M}+\mathrm{Na}]^{+}$. HR-MS (ESI) negative mode: found: 310.1660; calcd. for $\mathrm{C}_{16} \mathrm{H}_{25} \mathrm{NO}_{5}: 310.1654[\mathrm{M}-\mathrm{H}]^{-}$, positive mode: found: 334.1627 ; calcd. for $\mathrm{C}_{16} \mathrm{H}_{25} \mathrm{NO}_{5}: 334.1630[\mathrm{M}+\mathrm{Na}]^{+}$. 
Indicator Ia: According to the GP1, benzophenone 69 (41 $\mathrm{mg}, 0.16 \mathrm{mmol}), p$-toluene

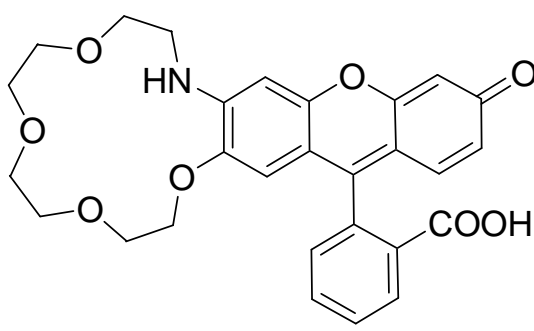
sulfonic acid monohydrate (1.6 $\mathrm{mg}, 8.4 \mu \mathrm{mol})$ and compound 42a (30 mg, $0.11 \mathrm{mmol}$ ) were heated for $48 \mathrm{~h}$ and gave, after purification by chromatography on silica gel $\left(20\right.$ g) with $\mathrm{CH}_{2} \mathrm{Cl}_{2} / \mathrm{MeOH}$ mixture $(10: 1 \rightarrow 3: 1)$ as an eluent, the title compound as a red-orange foam $(5.9 \mathrm{mg}$, 11\%). HPLC: $70 / 30 \rightarrow 0 / 100(\mathrm{~A} / \mathrm{B})$ in $25 \mathrm{~min}, 1 \mathrm{~mL} / \mathrm{min}, 25^{\circ} \mathrm{C}, t_{\mathrm{R}}=8.3 \mathrm{~min}$, detection at $254 \mathrm{~nm} .{ }^{1} \mathrm{H}$ NMR (300 MHz, $\left.\mathrm{CD}_{3} \mathrm{OD}\right): \delta=3.50\left(\mathrm{~m}, 2 \mathrm{H}, \mathrm{CH}_{2} \mathrm{~N}\right), 3.66\left(\mathrm{~m}, 8 \mathrm{H}, 4 \times \mathrm{CH}_{2}\right), 3.74$ (m, 2 H), 3.78 (m, 2 H), 3.84 (m, 2 H), 3.88 (m, 1 H, CHH), 4.03 (m, 1 H, CHH), 6.50 (s, 1 H, H-5), 6.63 (m, 2 H, H-2/4), 6.80 (s, 1 H), 7.14 (m, 1 H, H-1), 7.23 (m, 1 H), 7.60 (m, 2 H), $8.05(\mathrm{~m}, 1 \mathrm{H}) \mathrm{ppm} .{ }^{13} \mathrm{C}$ NMR $\left(125.7 \mathrm{MHz}, \mathrm{CD}_{3} \mathrm{OD}\right): \delta=43.6\left(\mathrm{CH}_{2} \mathrm{~N}\right), 68.8\left(\mathrm{CH}_{2}\right), 69.47$ $\left(\mathrm{CH}_{2}\right), 69.53\left(\mathrm{CH}_{2}\right), 70.7\left(\mathrm{CH}_{2}\right), 70.90\left(\mathrm{CH}_{2}\right), 70.93\left(2 \times \mathrm{CH}_{2}\right), 95.5(\mathrm{CH}), 103.8(\mathrm{CH}), 108.3$ $(\mathrm{CH}), 113.2(\mathrm{C}), 115.1(\mathrm{C}), 117.7(\mathrm{CH}), 124.3(\mathrm{CH}), 130.3(\mathrm{CH}), 130.4(\mathrm{CH}), 130.7(\mathrm{CH})$, 132.1 (CH), 135.3 (C), 136.3 (C), 140.5 (C), 145.7 (C), 149.2 (C), 155.0 (C), 160.0 (C), 173.5 (CO) ppm. ESI-MS, negative mode, m/z (rel. int., \%): 504 (100) [M - H] ${ }^{-}$. HR-MS (ESI, negative mode): found: 504.1663; calcd. for $\mathrm{C}_{28} \mathrm{H}_{27} \mathrm{NO}_{8}$ : $504.1658[\mathrm{M}-\mathrm{H}]^{-}$.

Indicator Ib: According to the GP5, compound 63c (125 mg, $0.31 \mathrm{mmol})$ with $10 \% \mathrm{Pd} / \mathrm{C}(23$<smiles></smiles>
$\mathrm{mg})$ in $\mathrm{AcOH}(2 \mathrm{~mL})$ gave the compound $\mathbf{4 2 b}$ as a brown oil (92 mg of crude product, 95\%). According to the GP1, benzophenone 69 (62 $\mathrm{mg}, 0.24 \mathrm{mmol}), p$-toluene sulfonic acid monohydrate $(2.0 \mathrm{mg}, 11 \mu \mathrm{mol})$ and compound $\mathbf{4 2 b}$ (50 $\mathrm{mg}$ of crude product) were heated for $36 \mathrm{~h}$ and gave, after purification by chromatography on silica gel $(20 \mathrm{~g})$ with $\mathrm{CH}_{2} \mathrm{Cl}_{2} / \mathrm{MeOH}$ mixture $(10: 1 \rightarrow 4: 1)$ as an eluent and preparative HPLC with reversed phase and gradient eluting with $\mathrm{H}_{2} \mathrm{O} / \mathrm{MeCN}$ mixture $\left(2: 8 \rightarrow 9: 1,40 \mathrm{~min}, 4 \mathrm{~mL} / \mathrm{min},, 25^{\circ} \mathrm{C}\right.$, $\left.t_{\mathrm{R}}=14.5 \mathrm{~min}\right)$, the title compound as a red foam $(4.8 \mathrm{mg}, 5.8 \%)$. HPLC: $70 / 30 \rightarrow 0 / 100(\mathrm{~A} / \mathrm{B})$ in $25 \mathrm{~min}, 1 \mathrm{~mL} / \mathrm{min}, 25^{\circ} \mathrm{C}, t_{\mathrm{R}}=7.2 \mathrm{~min}$, detection at $254 \mathrm{~nm} .{ }^{1} \mathrm{H}$ NMR $\left(600 \mathrm{MHz}, \mathrm{CD}_{3} \mathrm{OD}\right)$ : $\delta=1.32\left(\mathrm{~m}, 3 \mathrm{H}, \mathrm{CH}_{3}\right), 3.61\left(\mathrm{~m}, 8 \mathrm{H}, 4 \times \mathrm{CH}_{2}\right), 3.76\left(\mathrm{~m}, 4 \mathrm{H}, 2 \times \mathrm{CH}_{2}\right), 3.83\left(\mathrm{~m}, 6 \mathrm{H}, 3 \times \mathrm{CH}_{2}\right)$, 6.48 (s, $1 \mathrm{H}, \mathrm{H}-5), 6.87$ (m, $1 \mathrm{H}), 7.04$ (m, $2 \mathrm{H}), 7.30$ (m, $1 \mathrm{H}), 7.38\left(\mathrm{~d},{ }^{3} J=9.0,1 \mathrm{H}\right), 7.78$ (t, $\left.{ }^{3} J=9.0,1 \mathrm{H}\right), 7.85\left(\mathrm{t},{ }^{3} J=9.0,1 \mathrm{H}\right), 8.15(\mathrm{~m}, 1 \mathrm{H}) \mathrm{ppm} .{ }^{13} \mathrm{C} \mathrm{NMR}\left(125.7 \mathrm{MHz}, \mathrm{CD}_{3} \mathrm{OD}\right): \delta=$ $11.7\left(\underline{\mathrm{CH}}_{3} \mathrm{CH}_{2}\right), 45.4\left(\mathrm{CH}_{3} \underline{\mathrm{CH}_{2}}\right), 55.7\left(\mathrm{CH}_{2} \mathrm{~N}\right), 69.5\left(2 \times \mathrm{CH}_{2}\right), 70.1\left(\mathrm{CH}_{2}\right), 70.5\left(\mathrm{CH}_{2}\right), 70.8$ $\left(\mathrm{CH}_{2}\right), 71.1\left(\mathrm{CH}_{2}\right), 71.4\left(\mathrm{CH}_{2}\right), 103.2(\mathrm{CH}), 103.4(\mathrm{CH}), 109.3(\mathrm{CH}), 117.2(\mathrm{CH}), 119.4(\mathrm{C})$, 
$129.4(\mathrm{CH}), 130.7(\mathrm{CH}), 131.1(\mathrm{CH}), 131.6(\mathrm{CH}), 134.8(\mathrm{CH}), 150.3(\mathrm{C}), 156.4(\mathrm{C}), 162.7$ (C), 168.9 (CO) ppm. ESI-MS, negative mode, m/z (rel. int., \%): 532 (100) $[\mathrm{M}-\mathrm{H}]^{-}, 488$ (25) $\left[\mathrm{M}-\mathrm{H}-\mathrm{CO}_{2}\right]^{-}$, positive mode, $\mathrm{m} / \mathrm{z}$ (rel. int., \%): 534 (100) $[\mathrm{M}+\mathrm{H}]^{+}$. HR-MS (ESI, positive mode): found: 534.2104; calcd. for $\mathrm{C}_{30} \mathrm{H}_{31} \mathrm{NO}_{8}$ : $534.2128[\mathrm{M}+\mathrm{H}]^{+}$.

Compound Ic-Me•2/3TsOH: According to the GP1, benzophenone 69 (57 mg, $0.22 \mathrm{mmol}$ ),<smiles></smiles>
$p$-toluene sulfonic acid monohydrate $(4.0 \mathrm{mg}, 21 \mu \mathrm{mol})$ and compound $42 \mathrm{c}$ ( $52 \mathrm{mg}, 0.15 \mathrm{mmol}$ ) were heated for $48 \mathrm{~h}$ and gave, after purification by chromatography on silica gel $(20 \mathrm{~g})$ with $\mathrm{CH}_{2} \mathrm{Cl}_{2} / \mathrm{MeOH}$ mixture $(10: 1 \rightarrow 3: 1)$ as an eluent, the title compound as a red foam (19 $\mathrm{mg}, 18 \%)$. HPLC: $80 / 20 \rightarrow 10 / 90(\mathrm{~A} / \mathrm{B})$ in $40 \mathrm{~min}, 1 \mathrm{~mL} / \mathrm{min}, 25^{\circ} \mathrm{C}$, $t_{\mathrm{R}}=15.4 \mathrm{~min}$, detection at $254 \mathrm{~nm} .{ }^{1} \mathrm{H} \mathrm{NMR}\left(300 \mathrm{MHz}, \mathrm{CD}_{3} \mathrm{OD}\right): \delta=2.34(\mathrm{~s}, 2 \mathrm{H}=$ 2/3* $\left.{ }^{*} \mathrm{CH}_{3}(\mathrm{Ts})\right), 3.27\left(\mathrm{~m}, 2 \mathrm{H}, \mathrm{CH}_{2}\right), 3.54\left(\mathrm{~m}, 4 \mathrm{H}, 2 \times \mathrm{CH}_{2}\right), 3.64\left(\mathrm{~m}, 6 \mathrm{H}, 3 \times \mathrm{CH}_{2}\right), 3.76(\mathrm{~m}, 6 \mathrm{H}$ $\left.=\mathrm{COOCH}_{3}+\mathrm{CH} H+\mathrm{CH}_{2}\right), 3.95\left(\mathrm{~m}, 3 \mathrm{H}=\mathrm{CHH}+\underline{\mathrm{NCH}}_{2} \mathrm{COOH}\right), 6.25(\mathrm{~s}, 1 \mathrm{H}), 6.54(\mathrm{~m}, 2 \mathrm{H}$, $\mathrm{H}-2 / 5), 6.68$ (d, $\left.1 \mathrm{H},{ }^{4} J=2.2, \mathrm{H}-4\right), 7.21$ (m, 3 1/3 H, H-1 + 2/3*2 H(Ts) + H-7'), 7.60 (m, 3 $\left.1 / 3 \mathrm{H}, 2 / 3 * 2 \mathrm{H}(\mathrm{Ts})+\mathrm{H}-5^{\prime} / 6^{\prime}\right), 8.05\left(\mathrm{~d}, 1 \mathrm{H},{ }^{3} J=7.5, \mathrm{H}-4^{\prime}\right) \mathrm{ppm} .{ }^{13} \mathrm{C}$ NMR $(75.5 \mathrm{MHz}$, $\left.\mathrm{CD}_{3} \mathrm{OD}\right): \delta=21.3\left(\mathrm{Me}_{\mathrm{Ts}}\right), 53.2\left(\mathrm{CH}_{2} \mathrm{COOCH} \underline{3}_{3}\right), 56.2\left(\mathrm{CH}_{2} \underline{\mathrm{CH}}_{2} \mathrm{~N}\right), 56.9\left(\underline{\mathrm{CH}}_{2} \mathrm{COOCH}_{3}\right), 67.9$ $\left(\mathrm{CH}_{2}\right), 68.3\left(\mathrm{CH}_{2}\right), 68.8\left(\mathrm{CH}_{2}\right), 68.9\left(\mathrm{CH}_{2}\right), 69.2\left(2 \times \mathrm{CH}_{2}\right), 70.3\left(\mathrm{CH}_{2}\right), 103.5(\mathrm{CH}), 110.6$ $(\mathrm{CH}), 112.3(\mathrm{C}), 113.8(\mathrm{CH}), 115.9(\mathrm{C}), 125.2(\mathrm{CH}), 126.1(\mathrm{CH}), 127.0\left(2 \times \mathrm{CH}_{\mathrm{Ts}}\right), 127.9(\mathrm{C})$, $129.8\left(2 \times \mathrm{CH}_{\mathrm{Ts}}\right), 130.0(\mathrm{CH}), 131.4(\mathrm{CH}), 136.7(\mathrm{CH}), 141.6\left(\mathrm{C}_{\mathrm{Ts}}\right), 144.1(\mathrm{C}), 147.6\left(\mathrm{C}_{\mathrm{Ts}}\right)$, 149.5 (C), 153.9 (C), 154.0 (C), 161.4 (C), 171.3 (CO), 175.2 (CO) ppm. ESI-MS, negative mode, m/z (rel. int., \%): $576(100)[\mathrm{M}-\mathrm{H}]^{-}$, positive mode, m/z (rel. int., \%): 600 (100) $[\mathrm{M}+$ $\mathrm{Na}]^{+}$. HR-MS (ESI) negative mode: found: 576.1867; calcd. for $\mathrm{C}_{31} \mathrm{H}_{31} \mathrm{NO}_{10} \mathrm{~S} \cdot \mathrm{C}_{7} \mathrm{H}_{8} \mathrm{O}_{3} \mathrm{~S}$ : 576.1870 $[\mathrm{M}-\mathrm{H}]^{-}$, positive mode: found: 578.2022; calcd. for $\mathrm{C}_{31} \mathrm{H}_{31} \mathrm{NO}_{10} \mathrm{~S} \cdot \mathrm{C}_{7} \mathrm{H}_{8} \mathrm{O}_{3} \mathrm{~S}$ : $578.2026[\mathrm{M}+\mathrm{H}]^{+}$.

Indicator Ic: Compound Ic-Me•2/3TsOH (19 mg, $0.03 \mathrm{mmol})$ was dissolved in $\mathrm{MeOH}(1$<smiles></smiles>
$\mathrm{mL})$, then $\mathrm{K}_{2} \mathrm{CO}_{3}(8.9 \mathrm{mg}, 0.06 \mathrm{mmol})$ was added, and the mixture was stirred at room temperature for $1 \mathrm{~h}$. The solvent was removed in vacuo and the residue was dissolved in water $(2 \mathrm{~mL})$. This solution was passed through the column with an ion-exchange resin in $\mathrm{H}^{+}$-form. The water was removed by the freeze dryer. The residue was purified by 
preparative $\mathrm{HPLC}$ with reversed phase and gradient eluting with $\mathrm{H}_{2} \mathrm{O} / \mathrm{MeCN}$ mixture $(2: 8 \rightarrow$ 9:1, $40 \mathrm{~min}, 4 \mathrm{~mL} / \mathrm{min}, 25^{\circ} \mathrm{C}, t_{\mathrm{R}}=12.5 \mathrm{~min}$ ), and the title compound was isolated as redorange film $(1.5 \mathrm{mg}, 9.8 \%)$. HPLC: $80 / 20 \rightarrow 10 / 90(\mathrm{~A} / \mathrm{B})$ in $40 \mathrm{~min}, 1 \mathrm{~mL} / \mathrm{min}, 25^{\circ} \mathrm{C}, t_{\mathrm{R}}=$ $12.8 \mathrm{~min}$, detection at $254 \mathrm{~nm} .{ }^{1} \mathrm{H}$ NMR $\left(600 \mathrm{MHz}, \mathrm{CD}_{3} \mathrm{OD}\right): \delta=3.52(\mathrm{~m}, 2 \mathrm{H}$, $\left.\mathrm{NCH}_{2} \mathrm{COOH}\right), 3.62\left(\mathrm{~m}, 8 \mathrm{H}, 4 \times \mathrm{CH}_{2}\right), 3.73\left(\mathrm{~m}, 4 \mathrm{H}, 2 \times \mathrm{CH}_{2}\right), 3.82(\mathrm{~m}, 1 \mathrm{H}, \mathrm{CH} H), 3.95(\mathrm{~m}, 1$ H, CHH), $4.12\left(\mathrm{~m}, 2 \mathrm{H}, \mathrm{CH}_{2}\right), 6.28$ (s, $\left.1 \mathrm{H}, \mathrm{H}-5\right), 6.64(\mathrm{~m}, 1 \mathrm{H}), 6.72(\mathrm{~m}, 1 \mathrm{H}), 6.78(\mathrm{~s}, 1 \mathrm{H})$, $7.12(\mathrm{~m}, 1 \mathrm{H}), 7.26\left(\mathrm{~d},{ }^{3} J=9.0,1 \mathrm{H}\right), 7.73\left(\mathrm{t},{ }^{3} J=9.0,1 \mathrm{H}\right), 7.79\left(\mathrm{t},{ }^{3} J=9.0,1 \mathrm{H}\right), 8.09\left(\mathrm{~d},{ }^{3} J\right.$ $=9.0,1 \mathrm{H}) \mathrm{ppm} .{ }^{13} \mathrm{C} \mathrm{NMR}\left(125.7 \mathrm{MHz}, \mathrm{CD}_{3} \mathrm{OD}\right): \delta=55.5\left(\mathrm{CH}_{2} \underline{\mathrm{CH}}_{2} \mathrm{~N}\right), 57.2\left(\underline{\mathrm{CH}}_{2} \mathrm{COOCH}_{3}\right)$, 69.1 $\left(\mathrm{CH}_{2}\right), 69.3\left(\mathrm{CH}_{2}\right), 70.0\left(\mathrm{CH}_{2}\right), 70.1\left(\mathrm{CH}_{2}\right), 70.2\left(\mathrm{CH}_{2}\right), 70.3\left(\mathrm{CH}_{2}\right), 70.77\left(\mathrm{CH}_{2}\right), 70.81$ $\left(\mathrm{CH}_{2}\right), 103.3(\mathrm{CH}), 110.6(\mathrm{CH}), 114.9(\mathrm{CH}), 125.2(\mathrm{CH}), 126.1(\mathrm{CH}), 130.4(\mathrm{CH}), 131.3$ (CH), 133.9 (C), 135.9 (CH), 146.8 (C), 149.6 (C), 154.8 (C), 162.6 (C), 170.5 (CO) ppm. ESI-MS, negative mode, m/z (rel. int., \%): 584 (100) $[\mathrm{M}-2 \mathrm{H}+\mathrm{Na}]^{-}$. HR-MS (ESI, positive mode): found: 608.1495; calcd. for $\mathrm{C}_{30} \mathrm{H}_{29} \mathrm{NO}_{10}$ : $608.1503[\mathrm{M}-\mathrm{H}+2 \mathrm{Na}]^{+}$.

Indicator 70: According to the GP1, benzophenone 69 (81 $\mathrm{mg}, 0.31 \mathrm{mmol}), p$-toluene

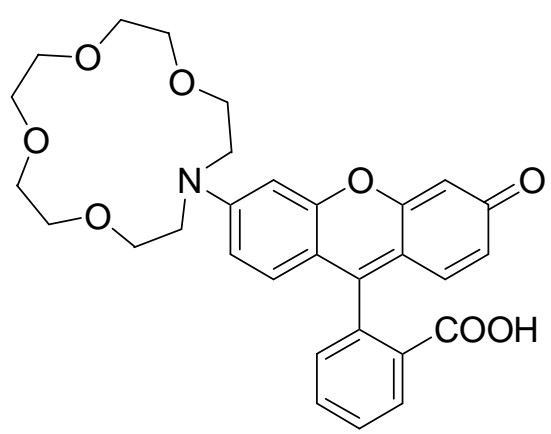
sulfonic acid monohydrate $(4.0 \mathrm{mg}, 21 \mu \mathrm{mol})$ and compound 68 ( $65 \mathrm{mg}, 0.21 \mathrm{mmol}$ ) were heated for $24 \mathrm{~h}$ and gave, after purification by chromatography on silica gel (50 g) with $\mathrm{CH}_{2} \mathrm{Cl}_{2} / \mathrm{MeOH}$ mixture $(10: 1 \rightarrow 5: 1)$ as an eluent followed by preparative HPLC and gradient elution with $\mathrm{H}_{2} \mathrm{O} / \mathrm{MeCN}$ mixture $(7: 3 \rightarrow 2: 8$, in $40 \mathrm{~min}, 16 \mathrm{~mL} / \mathrm{min}, 25$ $\left.{ }^{\circ} \mathrm{C}, t_{\mathrm{R}}=9.9 \mathrm{~min}\right)$, the title compound as a red foam $(5.6 \mathrm{mg}$, 5.0\%). HPLC: $80 / 20 \rightarrow 10 / 90(\mathrm{~A} / \mathrm{B})$ in $40 \mathrm{~min}, 1 \mathrm{~mL} / \mathrm{min}, 25{ }^{\circ} \mathrm{C}, t_{\mathrm{R}}=15.5 \mathrm{~min}$, detection at $500 \mathrm{~nm} .{ }^{1} \mathrm{H}$ NMR (300 MHz, $\left.\mathrm{CD}_{3} \mathrm{OD}\right): \delta=3.57$ (s, $4 \mathrm{H}, 2 \times \mathrm{CH}_{2}$ ), $3.62\left(\mathrm{~m}, 4 \mathrm{H}, 2 \times \mathrm{CH}_{2}\right.$ ), 3.67 (m, $\left.4 \mathrm{H}, 2 \times \mathrm{CH}_{2}\right), 3.88\left(\mathrm{~m}, 4 \mathrm{H}, 2 \times \mathrm{CH}_{2}\right), 3.94\left(\mathrm{~m}, 4 \mathrm{H}, 2 \times \mathrm{CH}_{2}\right), 6.96\left(\mathrm{dd},{ }^{3} J=8.4,{ }^{4} \mathrm{~J}=2.1,1\right.$ H, H-2), 7.13 (d, ${ }^{4} J=2.1,1$ H, H-4), 7.16 (m, 1 H), 7.21 (m, 3 H), 7.42 (m, 1 H), 7.84 (m, 2 $\mathrm{H}), 8.33(\mathrm{~m}, 1 \mathrm{H}) \mathrm{ppm} .{ }^{13} \mathrm{C} \mathrm{NMR}\left(125.7 \mathrm{MHz}, \mathrm{CD}_{3} \mathrm{OD}\right): \delta=55.2\left(2 \times \mathrm{CH}_{2} \mathrm{~N}\right), 69.6\left(2 \times \mathrm{CH}_{2}\right)$, $70.9\left(2 \times \mathrm{CH}_{2}\right), 71.6\left(2 \times \mathrm{CH}_{2}\right), 71.9\left(2 \times \mathrm{CH}_{2}\right), 98.5(\mathrm{CH}), 103.3(\mathrm{CH}), 116.3(\mathrm{C}), 116.4(\mathrm{C})$, $117.8(\mathrm{CH}), 118.2(\mathrm{CH}), 130.7(\mathrm{CH}), 131.6(\mathrm{CH}), 131.8(\mathrm{C}), 132.0(\mathrm{CH}), 132.5(\mathrm{CH}), 134.0$ (CH), $136.3(\mathrm{C}), 158.2$ (C), 159.4 (C), 159.6 (C), 168.1 (C), 168.6 (CO) ppm. ESI-MS, negative mode, m/z (rel. int., \%): 532 (100) $[\mathrm{M}-\mathrm{H}]^{-}$, positive mode, m/z (rel. int., \%): 556 (50) $[\mathrm{M}+\mathrm{Na}]^{+}, 572(100)[\mathrm{M}+\mathrm{K}]^{+}$. HR-MS (ESI, negative mode): found: 532.1973; calcd. for $\mathrm{C}_{30} \mathrm{H}_{31} \mathrm{NO}_{8}: 532.1971[\mathrm{M}-\mathrm{H}]^{-}$. 
1,2-Dimethyl-4,5-dinitrobenzene (77): According to the procedure for the preparation of 2,5-dinitrotoluene from 2-methyl-4-nitroaniline, ${ }^{[107]}$ 4,5-dimethyl-2-
nitroaniline (75) $(25 \mathrm{~g}, 0.15 \mathrm{~mol})$ was oxidized first with $\mathrm{K}_{2} \mathrm{~S}_{2} \mathrm{O}_{8}(122 \mathrm{~g}, 0.45$ and then with fuming nitric acid $(80 \mathrm{~mL}) .{ }^{[107]}$ The title compound was isolated as a colorless needles after recrystallization from $\mathrm{EtOH}(14.8 \mathrm{~g}, 50 \%)$; m. p. $118{ }^{\circ} \mathrm{C}\left(\right.$ Lit. $\left.^{[142]} 118{ }^{\circ} \mathrm{C}\right) .{ }^{1} \mathrm{H}$ NMR (300 MHz): $\delta=2.39$ (s, 3 H), 7.65 (s, 1 H) ppm. EI-MS, positive mode, m/z (rel. int., $\%): 196(100)[\mathrm{M}]^{+}$.

4,5-Dimethyl-2-nitrophenyl methyl ether (83): In the flask equipped with a reflux<smiles>COc1cc(C)c(C)cc1[N+](=O)[O-]</smiles>
condenser, sodium (62 mg, $2.7 \mathrm{mmol})$ was dissolved in $\mathrm{MeOH}(5 \mathrm{~mL})$. The solution was cooled to $0{ }^{\circ} \mathrm{C}$, and compound $77(0.51 \mathrm{~g}, 2.6 \mathrm{mmol})$ was added in small portions. The reaction mixture was stirred at $0{ }^{\circ} \mathrm{C}$ for $1 \mathrm{~h}$, and then heated at $65{ }^{\circ} \mathrm{C}$ for 2 days. The solvent was removed in vacuo, the residue was diluted with water $(10 \mathrm{~mL})$ and extracted with EtOAc $(3 \times 20 \mathrm{~mL})$. The combined organic solutions were washed with brine $(20 \mathrm{~mL})$ and dried. The solvent was removed in vacuo, the residue was purified by chromatography on silica gel $(50 \mathrm{~g})$ with $n$-hexane / EtOAc mixture (8:1) as an eluent, and the title compound was isolated as pale yellow needles after recrystallization from $n$-hexane $(0.38$ g, $81 \%)$; m. p. $75{ }^{\circ} \mathrm{C}\left(\right.$ Lit. $\left.^{[109]} 79{ }^{\circ} \mathrm{C}\right) .{ }^{1} \mathrm{H}$ NMR $(300 \mathrm{MHz}): \delta=2.22(\mathrm{~s}, 3 \mathrm{H}$, $\mathrm{CH}_{3} \mathrm{Ar}$ ), 2.29 (s, $\left.3 \mathrm{H}, \mathrm{CH}_{3} \mathrm{Ar}\right), 3.90$ (s, $\left.3 \mathrm{H}, \mathrm{CH}_{3} \mathrm{O}\right), 6.83(\mathrm{~s}, 1 \mathrm{H}), 7.67$ (s, $\left.1 \mathrm{H}\right) \mathrm{ppm} .{ }^{13} \mathrm{C}$ NMR (75.5 MHz): $\delta=18.6\left(\mathrm{CH}_{3} \mathrm{Ar}\right), 20.5\left(\mathrm{CH}_{3} \mathrm{Ar}\right), 56.5\left(\mathrm{CH}_{3} \mathrm{O}\right), 114.7(\mathrm{CH}), 126.7(\mathrm{CH})$, 128.8 (C), 136.9 (C), 144.6 (C), 151.2 (C) ppm. ESI-MS, positive mode, m/z (rel. int., \%): $204(51)[\mathrm{M}+\mathrm{Na}]^{+}, 385(100)[2 \mathrm{M}+\mathrm{Na}]^{+}$. HR-MS (ESI, positive mode): found: 204.0637; calcd. for $\mathrm{C}_{9} \mathrm{H}_{11} \mathrm{NO}_{3}: 204.0637[\mathrm{M}+\mathrm{H}]^{+}$.

4-Methoxy-5-nitrophthalic acid (79): According to procedure, described for the preparation $\mathrm{O}_{2} \mathrm{~N} \mathrm{COOH}$ of 4-methoxyphthalic acid (81) from 3,4-dimethylphenyl methyl ether MeO ${ }_{\mathrm{COOH}}^{(80)},{ }^{[108]}$ compound $83(5.0 \mathrm{~g}, 28 \mathrm{mmol})$ was oxidized with $\mathrm{KMnO}_{4}(24$ $\mathrm{g}, 0.15 \mathrm{~mol})$ under basic condition $[\mathrm{NaOH}(1.1 \mathrm{~g}, 28 \mathrm{mmol}) /$ water (400 $\mathrm{mL})$ ]. The title compound was isolated as a colourless solid after recrystallization from $\mathrm{H}_{2} \mathrm{O}$ (4.0 g, 74\%); m. p. $199{ }^{\circ} \mathrm{C}$ (Lit. $\left.{ }^{[109]} 201{ }^{\circ} \mathrm{C}\right) .{ }^{1} \mathrm{H}$ NMR (300 MHz, $\left.\mathrm{CD}_{3} \mathrm{OD}\right): \delta=4.03$ (s, $\left.3 \mathrm{H}\right)$, $7.45(\mathrm{~s}, 1 \mathrm{H}), 8.30(\mathrm{~s}, 1 \mathrm{H}) \mathrm{ppm} .{ }^{13} \mathrm{C} \mathrm{NMR}\left(75.5 \mathrm{MHz}, \mathrm{CD}_{3} \mathrm{OD}\right): \delta=57.8\left(\mathrm{CH}_{3} \mathrm{O}\right), 114.5$ (CH), $128.1(\mathrm{CH}), 140.9$ (C), 156.1 (C) ppm. ESI-MS, negative mode, m/z (rel. int., \%): 240 (100) $[\mathrm{M}-\mathrm{H}]^{-}$. 
4-amino-5-hydroxy- $N$-methylphthalimide (90) ${ }^{[113]}: 10 \% \mathrm{Pd} / \mathrm{C}(100 \mathrm{mg})$ and $\mathrm{MeOH}(10$<smiles>CN1C(=O)c2cc(N)c(O)cc2C1=O</smiles>

$\mathrm{mL}$ ) were placed into the Schlenk-flask equipped with a septum. The mixture was flushed with hydrogen and stirred vigorously to activate the catalyst. A solution of compound $89(0.50 \mathrm{~g}, 1.9 \mathrm{mmol})$ in $\mathrm{MeOH} / \mathrm{H}_{2} \mathrm{O}$ mixture $(3: 1 \mathrm{v} / \mathrm{v}, 100 \mathrm{~mL})$ was introduced through a septum. The solution was stirred under $\mathrm{H}_{2}$ for $4 \mathrm{~h}$ at room temperature. Then the hydrogen gas was replaced by argon, and the mixture was filtered through Celite ${ }^{\circledR}$. The filter-cake was washed with $\mathrm{MeOH}$ $(3 \times 50 \mathrm{~mL})$, and the solvent was removed in vacuo. The obtained mixture was diluted with toluene $(10 \mathrm{~mL})$, and the solvent was evaporated once more. The dry residue was dissolved in water $(15 \mathrm{~mL})$, and dry acetic acid $(2 \mathrm{~mL})$ was added to this solution. The title compound was filtered off, washed with water and air-dried. A yellow solid (285 mg, 78\%) was obtained; $\mathrm{m}$. p. $>300{ }^{\circ} \mathrm{C}\left(\right.$ Lit. $\left.^{[113]} 313-315{ }^{\circ} \mathrm{C}\right)$. HPLC: $70 / 30 \rightarrow 20 / 80(\mathrm{~A} / \mathrm{B})$ in $25 \mathrm{~min}, 1 \mathrm{~mL} / \mathrm{min}, 25^{\circ} \mathrm{C}$, $t_{\mathrm{R}}=4.2 \mathrm{~min}$, detection at $254 \mathrm{~nm} .{ }^{1} \mathrm{H}$ NMR (300 MHz, [D $]$ DMSO): $\delta=2.90(\mathrm{~s}, 3 \mathrm{H}), 5.67$ (br s, $\left.2 \mathrm{H}, \mathrm{NH}_{2}\right), 6.94(\mathrm{~s}, 1 \mathrm{H}), 6.97$ (s, $\left.1 \mathrm{H}\right)$ ppm. ESI-MS, negative mode, m/z (rel. int., \%): $191(48)[\mathrm{M}-\mathrm{H}]^{-}, 383(100)[2 \mathrm{M}-\mathrm{H}]^{-}$, positive mode, m/z (rel. int., \%): 215 (29) [M + $\mathrm{Na}]^{+}, 407(100)[2 \mathrm{M}+\mathrm{Na}]^{+}$. HR-MS (ESI) negative mode: found: 191.0462; calcd. for $\mathrm{C}_{9} \mathrm{H}_{8} \mathrm{~N}_{2} \mathrm{O}_{3}$ : 191.0457 [M $\left.-\mathrm{H}\right]^{-}$, positive mode: found: 215.0435 ; calcd. for $\mathrm{C}_{9} \mathrm{H}_{8} \mathrm{~N}_{2} \mathrm{O}_{3}$ : $215.0433[\mathrm{M}+\mathrm{Na}]^{+}$.

Crown ether 92: Method 1: To a solution of compound 90 (52 $\mathrm{mg}, 0.27 \mathrm{mmol})$ in a dry DMF<smiles></smiles>
$(2 \mathrm{~mL})$ in a dry Schlenk-flask equipped with a septum, NaI (81 $\mathrm{mg}, 0.54 \mathrm{mmol})$ and DIPEA $(143 \mu \mathrm{L}, \mathrm{d}=0.74,0.82 \mathrm{mmol})$ were added at room temperature, and the reaction mixture was vigorously stirred. Then compound 91 (112 mg, $0.27 \mathrm{mmol})$ was added, and the reaction mixture was heated at $90{ }^{\circ} \mathrm{C}$ for 1 day. All volatile materials were removed in vacuo, and the residue was diluted with $\mathrm{CH}_{2} \mathrm{Cl}_{2}(10 \mathrm{~mL})$. The organic solution was washed with water $(10 \mathrm{~mL})$ and dried. Dichloromethane was evaporated, and the residue was purified by chromatography on silica gel $(30 \mathrm{~g})$ with $\mathrm{CH}_{2} \mathrm{Cl}_{2} / \mathrm{MeCN}$ mixture (1:8) as an eluent. The title compound was isolated as a yellow solid $(14 \mathrm{mg}, 15 \%)$; m. p. $236{ }^{\circ} \mathrm{C}$ $(\mathrm{MeOH})$. HPLC: $70 / 30 \rightarrow 20 / 80(\mathrm{~A} / \mathrm{B})$ in $25 \mathrm{~min}, 1 \mathrm{~mL} / \mathrm{min}, 25^{\circ} \mathrm{C}, t_{\mathrm{R}}=11.9 \mathrm{~min}$, detection at $254 \mathrm{~nm} .{ }^{1} \mathrm{H}$ NMR $(300 \mathrm{MHz}): \delta=3.07(\mathrm{~s}, 3 \mathrm{H}), 3.32\left(\mathrm{~m}, 2 \mathrm{H}, \mathrm{CH}_{2} \mathrm{~N}\right), 3.65(\mathrm{~m}, 6 \mathrm{H}$, $\left.3 \times \mathrm{CH}_{2}\right), 3.71\left(\mathrm{~m}, 2 \mathrm{H}, \mathrm{CH}_{2}\right), 3.77\left(\mathrm{~m}, 2 \mathrm{H}, \mathrm{CH}_{2}\right), 3.86\left(\mathrm{~m}, 2 \mathrm{H}, \mathrm{CH}_{2}\right), 4.18\left(\mathrm{~m}, 2 \mathrm{H}, \mathrm{CH}_{2}\right)$, 5.64 (br s, $1 \mathrm{H}, \mathrm{NH}), 6.87(\mathrm{~s}, 1 \mathrm{H}), 7.06(\mathrm{~s}, 1 \mathrm{H}) \mathrm{ppm} .{ }^{13} \mathrm{C} \mathrm{NMR}(125.7 \mathrm{MHz}): \delta=23.7\left(\mathrm{CH}_{3}\right)$, 
$42.7\left(\mathrm{CH}_{2} \mathrm{~N}\right), 68.1\left(\mathrm{CH}_{2}\right), 68.2\left(\mathrm{CH}_{2}\right), 68.8\left(\mathrm{CH}_{2}\right), 69.8\left(\mathrm{CH}_{2}\right), 69.96\left(\mathrm{CH}_{2}\right), 70.02\left(\mathrm{CH}_{2}\right), 70.2$ $\left(\mathrm{CH}_{2}\right), 102.7(\mathrm{CH}), 104.5(\mathrm{CH}), 119.6(\mathrm{C}), 127.4(\mathrm{C}), 143.9(\mathrm{C}), 149.2(\mathrm{C}), 169.4(\mathrm{C}), 169.5$ (CO) ppm. ESI-MS, positive mode, m/z (rel. int., \%): $373(100)[\mathrm{M}+\mathrm{Na}]^{+}, 723$ (22) $[2 \mathrm{M}+$ $\mathrm{Na}]^{+}$. HR-MS (ESI, positive mode): found: 373.1373 ; calcd. for $\mathrm{C}_{17} \mathrm{H}_{22} \mathrm{~N}_{2} \mathrm{O}_{6}$ : $373.1370[\mathrm{M}+$ $\mathrm{Na}]^{+}$.

\section{2-[2-[2-[2-(2-Methyl-6-nitro-1H-isoindole-1,3(2H)-dion-5-yloxy)ethoxy]-ethoxy]-ethoxy]-}<smiles>CC(=O)OCCOCCOCCOCCOc1cc2c(cc1[N+](=O)[O-])C(=O)N(C)C2=O</smiles>
ethyl acetate (93-Ac): In a dry Schlenk-flask equipped with a septum and flushed with nitrogen, compound 89 $(1.0 \mathrm{~g}, 3.8 \mathrm{mmol})$ was dissolved in DMF (10 mL). Then KI (0.65 g, $3.9 \mathrm{mmol})$ and compound 54-Ac (1.2 g, $4.7 \mathrm{mmol})$ were added to the reaction mixture, and it was heated at $110^{\circ} \mathrm{C}$ for 3 days. The solvent was evaporated in vacuo, and the residue was diluted with $\mathrm{CH}_{2} \mathrm{Cl}_{2}(30 \mathrm{~mL})$ and mixed with water $(30 \mathrm{~mL})$. The organic layer was separated, washed with saturated aq. $\mathrm{Na}_{2} \mathrm{CO}_{3}(2 \times 50 \mathrm{~mL})$ and dried. The solvent was removed in vacuo, the residue was purified by chromatography on silica gel (100 g) with $\mathrm{CH}_{2} \mathrm{Cl}_{2} / \mathrm{MeOH}$ mixture (40:1) as an eluent, and the title compound was isolated as a pale yellow liquid $(0.70 \mathrm{~g}, 42 \%)$. ${ }^{1} \mathrm{H}$ NMR (300 MHz): $\delta=2.05$ (s, $\left.3 \mathrm{H}, \underline{\mathrm{CH}}_{3} \mathrm{CO}\right), 3.17$ (s, $\mathrm{NCH}_{3}$ ), 3.67 (m, $\left.10 \mathrm{H}\right), 3.91$ (m, $\left.2 \mathrm{H}\right), 4.18$ (m, $\left.2 \mathrm{H}\right), 4.39$ (m, $\left.2 \mathrm{H}\right), 7.58$ (s, $\left.1 \mathrm{H}\right), 8.15$ (s, 1 H) ppm. ${ }^{13} \mathrm{C}$ NMR (75.6 MHz): $\delta=20.9\left(\underline{\mathrm{CH}_{3}} \mathrm{CO}\right), 24.5\left(\mathrm{CH}_{3} \mathrm{~N}\right), 63.6\left(\underline{\mathrm{CH}}_{2} \mathrm{OAc}\right), 69.0\left(\mathrm{CH}_{2}\right)$, 69.1 $\left(\mathrm{CH}_{2}\right), 70.53\left(\mathrm{CH}_{2}\right), 70.57\left(\mathrm{CH}_{2}\right), 70.6\left(\mathrm{CH}_{2}\right), 70.7\left(\mathrm{CH}_{2}\right), 71.1\left(\mathrm{CH}_{2}\right), 109.6(\mathrm{CH}), 120.6$ $(\mathrm{CH}), 123.4(\mathrm{C}), 136.5(\mathrm{C}), 143.1(\mathrm{C}), 156.9(\mathrm{C}), 166.1(\mathrm{CO}), 166.4(\mathrm{CO}), 171.0\left(\mathrm{CH}_{3} \underline{\mathrm{CO}}\right)$ ppm. ESI-MS, positive mode, m/z (rel. int., \%): 463 (100) [M + Na $]^{+}$. HR-MS (ESI, positive mode): found: 463.1325 ; calcd. for $\mathrm{C}_{19} \mathrm{H}_{24} \mathrm{~N}_{2} \mathrm{O}_{10}$ : $463.1329[\mathrm{M}+\mathrm{Na}]^{+}$.

\section{5-[2-[2-(2-Hydroxyethoxy)-ethoxy]-ethoxy]-ethoxy-2-methyl-6-nitro-1H-isoindole-}

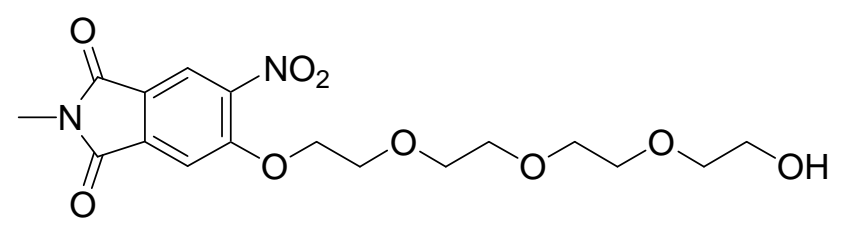
1,3(2H)-dion (93-H): Method 1: To a solution of compound 93-Ac (0.69 g, 1.6 $\mathrm{mmol})$ in $\mathrm{MeOH} / \mathrm{CH}_{2} \mathrm{Cl}_{2}$ mixture $(4: 1 \mathrm{v} / \mathrm{v}$, $100 \mathrm{~mL}), \mathrm{K}_{2} \mathrm{CO}_{3}(50 \mathrm{mg}, 0.36 \mathrm{mmol})$ was

added. The reaction mixture was stirred for $1 \mathrm{~h}$ at room temperature. The solvent was evaporated in vacuo. The residue was diluted with $\mathrm{CH}_{2} \mathrm{Cl}_{2}(30 \mathrm{~mL})$ and mixed with water (10 $\mathrm{mL}$ ). The organic solution was washed with brine and dried. The solvent was removed in vacuo, the residue was purified by chromatography on silica gel (70 g) with $\mathrm{CH}_{2} \mathrm{Cl}_{2} / \mathrm{MeOH}$ 
mixture (30:1) as an eluent, and the title compound was isolated as a pale yellow liquid $(0.31$ g, 49\%). HPLC: $70 / 30 \rightarrow 20 / 80(\mathrm{~A} / \mathrm{B})$ in $25 \mathrm{~min}, 1 \mathrm{~mL} / \mathrm{min}, 25^{\circ} \mathrm{C}, t_{\mathrm{R}}=8.6 \mathrm{~min}$, detection at 254 nm. ${ }^{1} \mathrm{H}$ NMR (300 MHz): $\delta=3.17\left(\mathrm{~s}, \mathrm{NCH}_{3}\right), 3.57\left(\mathrm{~m}, 2 \mathrm{H}, \underline{\mathrm{CH}}_{2} \mathrm{OH}\right), 3.67(\mathrm{~m}, 10 \mathrm{H})$, $3.91(\mathrm{~m}, 2 \mathrm{H}), 4.40(\mathrm{~m}, 2 \mathrm{H}), 7.61(\mathrm{~s}, 1 \mathrm{H}), 8.16$ (s, $1 \mathrm{H}) \mathrm{ppm} .{ }^{13} \mathrm{C}$ NMR $(75.6 \mathrm{MHz}): \delta=24.5$ $\left(\mathrm{CH}_{3} \mathrm{~N}\right), 61.7\left(\underline{\mathrm{CH}}_{2} \mathrm{OH}\right), 69.0\left(\mathrm{CH}_{2}\right), 70.2\left(\mathrm{CH}_{2}\right), 70.52\left(\mathrm{CH}_{2}\right), 70.58\left(\mathrm{CH}_{2}\right), 70.6\left(\mathrm{CH}_{2}\right), 71.1$ $\left(\mathrm{CH}_{2}\right), 72.4\left(\mathrm{CH}_{2}\right), 109.7(\mathrm{CH}), 120.6(\mathrm{CH}), 123.3(\mathrm{C}), 136.5(\mathrm{C}), 143.1(\mathrm{C}), 156.9(\mathrm{C}), 166.1$ (CO), 166.5 (CO) ppm. ESI-MS, positive mode, m/z (rel. int., \%): 421 (100) [M+ Na] $]^{+}, 819$ (6) $[2 \mathrm{M}+\mathrm{Na}]^{+}$. HR-MS (ESI, positive mode): found: 421.1212; calcd. for $\mathrm{C}_{17} \mathrm{H}_{22} \mathrm{~N}_{2} \mathrm{O}_{9}$ : $421.1223[\mathrm{M}+\mathrm{Na}]^{+}$.

2-[2-[2-(2-Iodoethoxy)-ethoxy]-ethoxy]-ethyl trityl ether (96): To a solution of compound $\sim \sim_{\mathrm{OTr}} 95(1.0 \mathrm{~g}, 1.9 \mathrm{mmol})$ in dry THF $(10 \mathrm{~mL})$, in a dry Schlenk-flask equipped with a septum and flushed with nitrogen, LiI (0.52 g, $3.9 \mathrm{mmol})$ was added. The reaction mixture was heated at $40{ }^{\circ} \mathrm{C}$ for $1 \mathrm{~h}$, diluted with diethyl ether $(25 \mathrm{~mL})$, washed with water $(2 \times 25 \mathrm{~mL})$ and dried. The solvent was removed in vacuo, the title compound was isolated as a brownish liquid (0.94 g, 98\%). HPLC: $50 / 50 \rightarrow 0 / 100(\mathrm{~A} / \mathrm{B})$ in $25 \mathrm{~min}, 1 \mathrm{~mL} / \mathrm{min}, 25^{\circ} \mathrm{C}, t_{\mathrm{R}}=19.0 \mathrm{~min}$, detection at $254 \mathrm{~nm} .{ }^{1} \mathrm{H}$ NMR (300 MHz): $\delta=3.24(\mathrm{~m}, 2 \mathrm{H}), 3.68\left(\mathrm{~m}, 12 \mathrm{H}, 6 \times \mathrm{CH}_{2}\right), 3.74\left(\mathrm{~m}, 2 \mathrm{H}, \mathrm{CH}_{2} \mathrm{OTr}\right), 7.28$ $(\mathrm{m}, 10 \mathrm{H}), 7.37(\mathrm{~m}, 5 \mathrm{H}) \mathrm{ppm} .{ }^{13} \mathrm{C} \mathrm{NMR}(75.5 \mathrm{MHz}): \delta=2.9\left(\mathrm{CH}_{2} \mathrm{I}\right), 63.3\left(\underline{\mathrm{CH}}_{2} \mathrm{OTr}\right), 70.2$ $\left(\mathrm{CH}_{2}\right), 70.7\left(2 \times \mathrm{CH}_{2}\right), 70.8\left(2 \times \mathrm{CH}_{2}\right), 72.0\left(\mathrm{CH}_{2}\right), 86.5\left(\mathrm{CPh}_{3}\right), 126.9(\mathrm{CH}), 127.7(\mathrm{CH}), 128.7$ (CH), 144.1 (C) ppm. ESI-MS, positive mode, m/z (rel. int., \%): $569(100)[\mathrm{M}+\mathrm{Na}]^{+}$. HRMS (ESI, positive mode): found: 569.1163; calcd. for $\mathrm{C}_{27} \mathrm{H}_{31} \mathrm{IO}_{4}: 569.1165[\mathrm{M}+\mathrm{Na}]^{+}$.

\section{2-Methyl-6-nitro-5-[2-[2-(2-trityloxyethoxy)-ethoxy]-ethoxy]-ethoxy-1H-isoindole-}

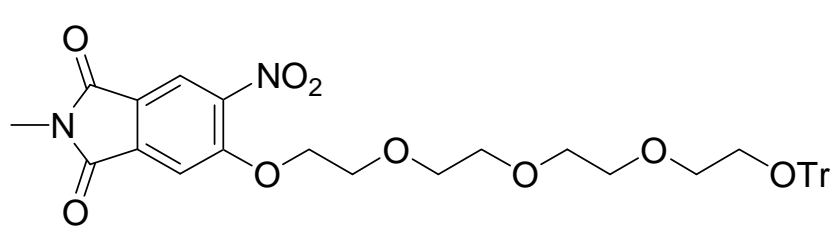

1,3(2H)-dion (93-Tr): In a dry Schlenkflask equipped with a septum and flushed with nitrogen, compound 89 (1.2 g, 4.6 mmol) was dissolved in DMF (15 mL).

Then compound 96 ( $4.4 \mathrm{~g}, 8.1 \mathrm{mmol})$ was added, and the mixture was heated at $70{ }^{\circ} \mathrm{C}$ for 5 days. The solvent was evaporated in vacuo, the residue was diluted with $\mathrm{CHCl}_{3}(50 \mathrm{~mL})$ and mixed with water $(50 \mathrm{~mL})$. The organic layer was separated, washed with saturated aq. $\mathrm{Na}_{2} \mathrm{CO}_{3}(2 \times 50 \mathrm{~mL})$, brine and dried. The solvent was removed in vacuo, the residue was purified by chromatography on silica gel (130 g) with $n$-hexane / EtOAc mixture $(1: 1)$ as an eluent, and the title compound was isolated as a pale yellow resin (1.7 g, 58\%). HPLC: 70/30 
$\rightarrow 20 / 80(\mathrm{~A} / \mathrm{B})$ in $25 \mathrm{~min}, 1 \mathrm{~mL} / \mathrm{min}, 25{ }^{\circ} \mathrm{C}, t_{\mathrm{R}}=9.2 \mathrm{~min}$, detection at $254 \mathrm{~nm} .{ }^{1} \mathrm{H} \mathrm{NMR}(300$ MHz): $\delta=3.17\left(\mathrm{~s}, \mathrm{NCH}_{3}\right), 3.21\left(\mathrm{CH}_{2} \mathrm{OTr}\right), 3.67(\mathrm{~m}, 10 \mathrm{H}), 3.88(\mathrm{~m}, 2 \mathrm{H}), 4.26(\mathrm{~m}, 2 \mathrm{H}), 7.23$ $(\mathrm{m}, 10 \mathrm{H}), 7.44(\mathrm{~m}, 5 \mathrm{H}), 7.48(\mathrm{~s}, 1 \mathrm{H}), 8.14(\mathrm{~s}, 1 \mathrm{H}) \mathrm{ppm} .{ }^{13} \mathrm{C}$ NMR $(75.5 \mathrm{MHz}): \delta=24.4$ $\left(\mathrm{CH}_{3} \mathrm{~N}\right), 63.3\left(\underline{\mathrm{CH}}_{2} \mathrm{OTr}\right), 69.0\left(\mathrm{CH}_{2}\right), 70.61\left(\mathrm{CH}_{2}\right), 70.65\left(\mathrm{CH}_{2}\right), 70.73\left(\mathrm{CH}_{2}\right), 70.76\left(\mathrm{CH}_{2}\right)$, $70.78\left(\mathrm{CH}_{2}\right), 71.1\left(\mathrm{CH}_{2}\right), 86.5\left(\underline{\mathrm{CPh}}_{3}\right), 109.6(\mathrm{CH}), 120.5(\mathrm{CH}), 123.3(\mathrm{C}), 126.9(\mathrm{CH}), 127.7$ (CH), $128.7(\mathrm{CH}), 136.5$ (C), 144.1 (C), 146.8 (C), 156.9 (C), 166.1 (CO), 166.4 (CO) ppm. ESI-MS, negative mode, m/z (rel. int., \%): 657 (100) [M+OH] $]^{-}$, positive mode, m/z (rel. int., \%): 663 (100) $[\mathrm{M}+\mathrm{Na}]^{+}$. HR-MS (ESI, positive mode): found: 663.2315; calcd. for $\mathrm{C}_{36} \mathrm{H}_{36} \mathrm{~N}_{2} \mathrm{O}_{9}: 663.2319[\mathrm{M}+\mathrm{Na}]^{+}$.

\section{5-[2-[2-(2-Hydroxyethoxy)-ethoxy]-ethoxy]-ethoxy-2-methyl-6-nitro-1H-isoindole-}<smiles>CN1C(=O)c2cc(OCCOCCOCCOCCO)c([N+](=O)[O-])cc2C1=O</smiles>
1,3(2H)-dion (93-H): Method 2 (see Method 1 on page 154): To a solution of compound 93-Tr $(100 \mathrm{mg}, 0.16 \mathrm{mmol})$ in $\mathrm{MeOH} / \mathrm{CH}_{2} \mathrm{Cl}_{2}$ mixture $(4: 1 \mathrm{v} / \mathrm{v}, 5 \mathrm{~mL}), 2$

M solution of $\mathrm{BF}_{3} \cdot \mathrm{Et}_{2} \mathrm{O}$ in $\mathrm{MeOH}(100 \mu \mathrm{L}, 0.2 \mathrm{mmol})$ was added. The reaction mixture was stirred overnight at room temperature. The solvent was evaporated in vacuo, and the residue was diluted with $\mathrm{CH}_{2} \mathrm{Cl}_{2}(30 \mathrm{~mL})$. The organic solution was washed with water and with brine, and dried. The solvent was removed in vacuo, the residue was purified by chromatography on silica gel $(20 \mathrm{~g})$ with $\mathrm{CH}_{2} \mathrm{Cl}_{2} / \mathrm{MeOH}$ mixture (50:1) as an eluent, and the title compound was isolated as a pale yellow resin (61 mg, 96\%). The NMR and mass spectra were reported above.

\section{5-Amino-6-[2-[2-(2-hydroxyethoxy)-ethoxy]-ethoxy]-ethoxy-2-methyl-1H-isoindole-}

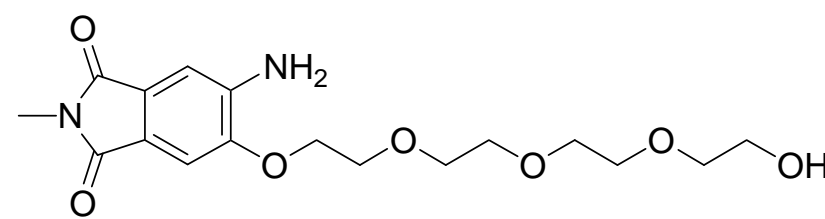

1,3(2H)-dion (97): According to the GP2, compound 93-H (300 mg, $0.75 \mathrm{mmol})$ was reduced in $\mathrm{MeOH}$ and gave the title compound as a yellow oil (260 $\mathrm{mg}, 94 \%)$.

HPLC: $50 / 50 \rightarrow 0 / 100(\mathrm{~A} / \mathrm{B})$ in $25 \mathrm{~min}, 1 \mathrm{~mL} / \mathrm{min}, 25^{\circ} \mathrm{C}, t_{\mathrm{R}}=2.7 \mathrm{~min}$, detection at $254 \mathrm{~nm}$. ${ }^{1} \mathrm{H}$ NMR (300 MHz): $\delta=3.06(\mathrm{~s}, 3 \mathrm{H}), 3.58(\mathrm{~m}, 2 \mathrm{H}), 3.68\left(\mathrm{~m}, 10 \mathrm{H}, 5 \times \mathrm{CH}_{2}\right), 3.85(\mathrm{~m}, 2 \mathrm{H}$, $\left.\mathrm{CH}_{2}\right), 4.21(\mathrm{~m}, 2 \mathrm{H}), 4.80\left(\mathrm{~s}, 1 \mathrm{H}, \mathrm{NH}_{2}\right), 7.01(\mathrm{~s}, 1 \mathrm{H}), 7.13(\mathrm{~s}, 1 \mathrm{H}) \mathrm{ppm} .{ }^{13} \mathrm{C}$ NMR $(75.6$ MHz): $\delta=23.7\left(\mathrm{CH}_{3} \mathrm{~N}\right), 61.6\left(\underline{\mathrm{CH}_{2}} \mathrm{OAr}\right), 68.6\left(\mathrm{CH}_{2}\right), 69.3\left(\mathrm{CH}_{2}\right), 70.2\left(\mathrm{CH}_{2}\right), 70.5\left(\mathrm{CH}_{2}\right)$, $70.59\left(\mathrm{CH}_{2}\right), 70.64\left(\mathrm{CH}_{2}\right), 72.5\left(\mathrm{CH}_{2}\right), 106.5(\mathrm{CH}), 107.9(\mathrm{CH}), 121.3(\mathrm{C}), 127.2(\mathrm{C}), 143.0$ (C), 149.2 (C), 169.1 (CO), 169.2 (CO) ppm. ESI-MS, negative mode, m/z (rel. int., \%): 367 
(100) $[\mathrm{M}-\mathrm{H}]^{-}$, positive mode, $\mathrm{m} / \mathrm{z}$ (rel. int., \%): $391(100)[\mathrm{M}+\mathrm{Na}]^{+}, 759(56)[2 \mathrm{M}+\mathrm{Na}]^{+}$. HR-MS (ESI) negative mode: found: 367.1500; calcd. for $\mathrm{C}_{17} \mathrm{H}_{24} \mathrm{~N}_{2} \mathrm{O}_{7}: 367.1505[\mathrm{M}-\mathrm{H}]^{-}$, positive mode: found: 391.1470 ; calcd. for $\mathrm{C}_{17} \mathrm{H}_{24} \mathrm{~N}_{2} \mathrm{O}_{7}$ : $391.1481[\mathrm{M}+\mathrm{Na}]^{+}$.

\section{2-[2-[2-(2-(2-Methyl-6-tosylamino-1H-isoindole-1,3(2H)-dion-5-yloxy)ethoxy)-ethoxy]-}

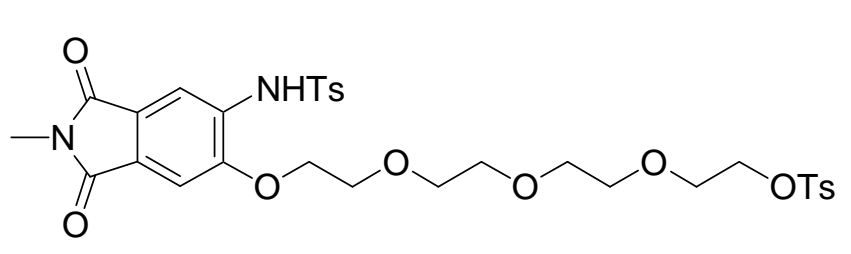
ethoxy]-ethyl tosylate (98): According to the GP3, compound 97 (1.6 g, $4.3 \mathrm{mmol})$ and tosyl chloride $(1.7 \mathrm{~g}, 8.9 \mathrm{mmol})$ gave, after purification by chromatography on silica gel (200 g) with $\mathrm{CH}_{2} \mathrm{Cl}_{2} / \mathrm{MeOH}$ mixture (40:1) as an eluent, the title compound as a colorless oil (2.1 g, 72\%). HPLC: 50/50 $\rightarrow$ 0/100 (A/B) in $25 \mathrm{~min}, 1 \mathrm{~mL} / \mathrm{min}, 25^{\circ} \mathrm{C}, t_{\mathrm{R}}=11.8$ min, detection at $254 \mathrm{~nm} .{ }^{1} \mathrm{H}$ NMR (300 MHz): $\delta=2.22\left(\mathrm{~s}, 3 \mathrm{H}, \mathrm{CH}_{3}\right), 2.41\left(\mathrm{~s}, 3 \mathrm{H}, \mathrm{CH}_{3}\right)$, $3.17\left(\mathrm{~s}, 3 \mathrm{H}, \mathrm{NCH}_{3}\right), 3.66\left(\mathrm{~m}, 10 \mathrm{H}, 5 \times \mathrm{CH}_{2}\right), 3.71(\mathrm{~m}, 2 \mathrm{H}), 4.14\left(\mathrm{~m}, 4 \mathrm{H}, 2 \times \mathrm{CH}_{2}\right), 7.21(\mathrm{~m}, 2$ $\left.\mathrm{H}_{\mathrm{TsNH}}+1 \mathrm{H}\right), 7.30\left(\mathrm{~d},{ }^{3} J=8.4,2 \mathrm{H}_{\mathrm{TsO}}\right), 7.74\left(\mathrm{~d},{ }^{3} J=8.1,2 \mathrm{H}_{\mathrm{TsNH}}\right), 7.76\left(\mathrm{~d},{ }^{3} J=8.4,2 \mathrm{H}_{\mathrm{TsO}}\right)$, $7.91(\mathrm{~s}, 1 \mathrm{H}), 8.12$ (s, $1 \mathrm{H}, \mathrm{NHTs}) \mathrm{ppm} .{ }^{13} \mathrm{C} \mathrm{NMR}(75.5 \mathrm{MHz}): \delta=21.5\left(\mathrm{CH}_{3}\right), 21.6\left(\mathrm{CH}_{3}\right)$, $24.0\left(\mathrm{CH}_{3} \mathrm{~N}\right), 68.7\left(\underline{\mathrm{CH}_{2}} \mathrm{OTs}\right), 68.9\left(\mathrm{CH}_{2}\right), 69.3\left(\mathrm{CH}_{2}\right), 69.7\left(\mathrm{CH}_{2}\right), 70.5\left(\mathrm{CH}_{2}\right), 70.65\left(\mathrm{CH}_{2}\right)$, $70.73\left(\mathrm{CH}_{2}\right), 70.76\left(\mathrm{CH}_{2}\right), 107.2(\mathrm{CH}), 113.6(\mathrm{CH}), 125.8(\mathrm{C}), 127.3(\mathrm{CH}), 127.9(\mathrm{CH}), 128.4$ (C), $129.76(\mathrm{CH}), 129.82(\mathrm{CH}), 132.4(\mathrm{C}), 132.9$ (C), 136.0 (C), 144.3 (C), 144.8 (C), 152.0 (C), 167.8 (CO), 168.0 (CO) ppm. ESI-MS, negative mode, m/z (rel. int., \%): 675 (100) [M $\mathrm{H}]^{-}$, positive mode, m/z (rel. int., \%): 699 (100) $[\mathrm{M}+\mathrm{Na}]^{+}$. HR-MS (ESI, positive mode): found: 699.1658; calcd. for $\mathrm{C}_{31} \mathrm{H}_{36} \mathrm{~N}_{2} \mathrm{O}_{11} \mathrm{~S}_{2}: 699.1653[\mathrm{M}+\mathrm{Na}]^{+}$.

Crown ether 99: According to the GP4, compound 98 (5.6 g, $8.3 \mathrm{mmol})$ and caesium

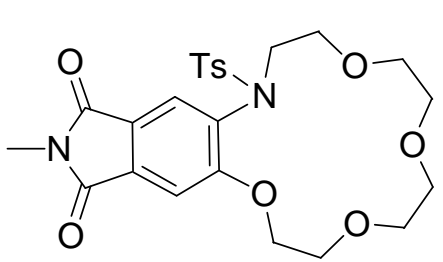
carbonate $(3.0 \mathrm{~g}, 9.2 \mathrm{mmol})$ in DMF $(80 \mathrm{~mL})$ gave, after purification by chromatography on silica gel $(200 \mathrm{~g})$ with $\mathrm{CH}_{2} \mathrm{Cl}_{2}$ / $\mathrm{MeOH}$ mixture (50:1) as an eluent, the title compound as a colourless oil $(0.90 \mathrm{~g}, 21 \%)$. HPLC: $50 / 50 \rightarrow 0 / 100(\mathrm{~A} / \mathrm{B})$ in 25 $\min , 1 \mathrm{~mL} / \mathrm{min}, 25^{\circ} \mathrm{C}, t_{\mathrm{R}}=8.9 \mathrm{~min}$, detection at $254 \mathrm{~nm} .{ }^{1} \mathrm{H} \mathrm{NMR}(300 \mathrm{MHz}): \delta=2.40(\mathrm{~s}, 3$ $\left.\mathrm{H}, \mathrm{CH}_{3}\right), 3.13\left(\mathrm{~s}, 3 \mathrm{H}, \mathrm{NCH}_{3}\right), 3.46\left(\mathrm{~m}, 4 \mathrm{H}, 2 \times \mathrm{CH}_{2}\right), 3.52\left(\mathrm{~m}, 4 \mathrm{H}, 2 \times \mathrm{CH}_{2}\right), 3.58(\mathrm{~m}, 2 \mathrm{H}$, $\left.\mathrm{CH}_{2}\right) 3.66\left(\mathrm{~m}, 4 \mathrm{H}, 2 \times \mathrm{CH}_{2}\right), 3.95\left(\mathrm{~m}, 2 \mathrm{H}, \mathrm{CH}_{2}\right), 7.24\left(\mathrm{~m}, 2 \mathrm{H}_{\mathrm{TsNH}}+1 \mathrm{H}\right), 7.57\left(\mathrm{~d},{ }^{3} \mathrm{~J}=8.1,2\right.$ $\left.\mathrm{H}_{\mathrm{TsN}}\right), 7.65(\mathrm{~s}, 1 \mathrm{H}) \mathrm{ppm} .{ }^{13} \mathrm{C} \mathrm{NMR}(75.5 \mathrm{MHz}): \delta=21.5\left(\mathrm{CH}_{3}\right), 24.1\left(\mathrm{CH}_{3} \mathrm{~N}\right), 50.2\left(\mathrm{CH}_{2}\right)$, $67.9\left(\mathrm{CH}_{2}\right), 68.3\left(\mathrm{CH}_{2}\right), 69.9\left(\mathrm{CH}_{2}\right), 70.0\left(\mathrm{CH}_{2}\right), 70.6\left(\mathrm{CH}_{2}\right), 70.8\left(\mathrm{CH}_{2}\right), 71.3\left(\mathrm{CH}_{2}\right), 106.9$ $(\mathrm{CH}), 123.8(\mathrm{C}), 127.5(\mathrm{CH}), 127.7(2 \times \mathrm{CH}), 129.3(2 \times \mathrm{CH}), 133.2(\mathrm{C}), 133.8(\mathrm{C}), 136.7(\mathrm{C})$, 143.4 (C), 160.8 (C), 167.5 (CO), 167.8 (CO) ppm. ESI-MS, negative mode, m/z (rel. int., 
\%): $539(100)[\mathrm{M}+\mathrm{Cl}]^{-}$, positive mode, $\mathrm{m} / \mathrm{z}$ (rel. int., \%): $527(100)[\mathrm{M}+\mathrm{Na}]^{+}, 1031$ (18) $[2 \mathrm{M}+\mathrm{Na}]^{+}$. HR-MS (ESI, positive mode): found: 527.1464; calcd. for $\mathrm{C}_{24} \mathrm{H}_{28} \mathrm{~N}_{2} \mathrm{O}_{8} \mathrm{~S}$ : $527.1464[\mathrm{M}+\mathrm{Na}]^{+}$.

2-[2-[2-[2-(2-Methyl-6-nitro-1H-isoindole-1,3(2H)-dion-5-yloxy)ethoxy]-ethoxy]-ethoxy]-<smiles>CN1C(=O)c2cc(OCCOCCOCCOCC[Se-])c([N+](=O)[O-])cc2C1=O</smilesethyl tosylate (101): According to the GP3, compound 93-H (1.1 g, $2.8 \mathrm{mmol})$ and tosyl chloride $(0.78 \mathrm{~g}, 4.1 \mathrm{mmol})$ gave, after purification by chromatography on silica gel $(100 \mathrm{~g})$ with $\mathrm{CH}_{2} \mathrm{Cl}_{2} /$ EtOAc mixture $(2: 1 \rightarrow 1: 1)$ as an eluent, the title compound as a pale yellow oil $(0.80 \mathrm{~g}, 52 \%)$. ${ }^{1} \mathrm{H}$ NMR $(300 \mathrm{MHz}): \delta=2.41\left(\mathrm{~s}, 3 \mathrm{H}, \mathrm{CH}_{3}\right), 3.18(\mathrm{~s}, 3 \mathrm{H}$, $\left.\mathrm{NCH}_{3}\right), 3.66\left(\mathrm{~m}, 10 \mathrm{H}, 5 \times \mathrm{CH}_{2}\right), 3.91\left(\mathrm{~m}, 2 \mathrm{H}, \mathrm{CH}_{2}\right), 4.12\left(\mathrm{~m}, 2 \mathrm{H}, \mathrm{CH}_{2}\right), 4.38\left(\mathrm{~m}, 2 \mathrm{H}, \mathrm{CH}_{2}\right)$, $7.30\left(\mathrm{~d},{ }^{3} J=8.4,2 \mathrm{H}_{\mathrm{TsO}}\right), 7.58(\mathrm{~s}, 1 \mathrm{H}), 7.74\left(\mathrm{~d},{ }^{3} J=8.4,2 \mathrm{H}_{\mathrm{TsO}}\right), 8.15(\mathrm{~s}, 1 \mathrm{H}) \mathrm{ppm} .{ }^{13} \mathrm{C}$ NMR (75.5 MHz): $\delta=21.6\left(\mathrm{CH}_{3}\right), 24.5\left(\mathrm{CH}_{3} \mathrm{~N}\right), 68.6\left(\underline{\left.\mathrm{CH}_{2} \mathrm{OTs}\right),} 69.0\left(\mathrm{CH}_{2}\right), 69.2\left(\mathrm{CH}_{2}\right), 70.5\right.$ $\left(\mathrm{CH}_{2}\right), 70.67\left(\mathrm{CH}_{2}\right), 70.72\left(2 \times \mathrm{CH}_{2}\right), 71.1\left(\mathrm{CH}_{2}\right), 109.7(\mathrm{CH}), 120.5(\mathrm{CH}), 123.4(\mathrm{C}), 127.9$ (CH), $129.8(\mathrm{CH}), 133.0(\mathrm{C}), 136.6(\mathrm{C}), 143.1$ (C), 144.8 (C), 156.9 (C), 166.1 (CO), 166.4 (CO) ppm. ESI-MS, positive mode, m/z (rel. int., \%): 575 (100) $[\mathrm{M}+\mathrm{Na}]^{+}$. HR-MS (ESI, positive mode): found: 575.1307 ; calcd. for $\mathrm{C}_{24} \mathrm{H}_{28} \mathrm{~N}_{2} \mathrm{O}_{11} \mathrm{~S}: 575.1312[\mathrm{M}+\mathrm{Na}]^{+}$.

\section{2-[2-[2-[2-(6-Amino-2-methyl-1H-isoindole-1,3(2H)-dion-5-yloxy)ethoxy]-ethoxy]-}

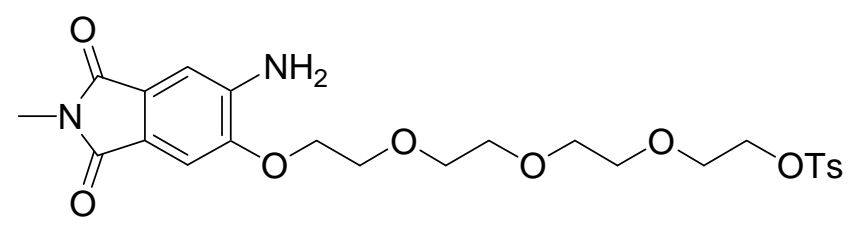

ethoxy]-ethyl tosylate (100): According to the GP2, compound 101 (150 mg, 0.27 $\mathrm{mmol}$ ) was reduced in THF and gave the title compound as a yellow oil (125 mg, 89\%). HPLC: $70 / 30 \rightarrow 20 / 80(\mathrm{~A} / \mathrm{B})$ in $25 \mathrm{~min}, 1 \mathrm{~mL} / \mathrm{min}, 25{ }^{\circ} \mathrm{C}, t_{\mathrm{R}}=16.6 \mathrm{~min}$, detection at $254 \mathrm{~nm} .{ }^{1} \mathrm{H}$ NMR (300 MHz): $\delta=2.40\left(\mathrm{~s}, 3 \mathrm{H}, \mathrm{CH}_{3}\right), 3.06(\mathrm{~s}, 3 \mathrm{H}), 3.64\left(\mathrm{~m}, 10 \mathrm{H}, 5 \times \mathrm{CH}_{2}\right)$, $3.86\left(\mathrm{~m}, 2 \mathrm{H}, \mathrm{CH}_{2}\right), 4.12(\mathrm{~m}, 2 \mathrm{H}), 4.21(\mathrm{~m}, 2 \mathrm{H}), 7.02(\mathrm{~s}, 1 \mathrm{H}), 7.14(\mathrm{~s}, 1 \mathrm{H}), 7.30\left(\mathrm{~d},{ }^{3} J=8.4\right.$, $\left.2 \mathrm{H}_{\mathrm{TsO}}\right), 7.76\left(\mathrm{~d},{ }^{3} J=8.4,2 \mathrm{H}_{\mathrm{TsO}}\right)$ ppm. ${ }^{13} \mathrm{C} \mathrm{NMR}(75.6 \mathrm{MHz}): \delta=21.6\left(\mathrm{CH}_{3}\right), 23.7\left(\mathrm{CH}_{3} \mathrm{~N}\right)$, $68.68\left(\underline{\mathrm{CH}_{2}} \mathrm{OTs}\right), 68.74\left(\mathrm{CH}_{2}\right), 69.2\left(\mathrm{CH}_{2}\right), 69.3\left(\mathrm{CH}_{2}\right), 70.5\left(\mathrm{CH}_{2}\right), 70.58\left(\mathrm{CH}_{2}\right), 70.63\left(\mathrm{CH}_{2}\right)$, $70.8\left(\mathrm{CH}_{2}\right), 106.5(\mathrm{CH}), 108.0(\mathrm{CH}), 121.6(\mathrm{C}), 127.1(\mathrm{C}), 127.9(2 \times \mathrm{CH}), 129.8(2 \times \mathrm{CH})$, 132.9 (C), 142.5 (C), 144.8 (C), 149.2 (C), 168.9 (CO), 169.1 (CO) ppm. ESI-MS, negative mode, m/z (rel. int., \%): 521 (100) [M - H] $]^{-}$, positive mode, m/z (rel. int., \%): 545 (100) [M + $\mathrm{Na}]^{+}, 1067$ (17) $[2 \mathrm{M}+\mathrm{Na}]^{+}$. HR-MS (ESI, positive mode): found: 545.1567; calcd. for $\mathrm{C}_{24} \mathrm{H}_{30} \mathrm{~N}_{2} \mathrm{O}_{9} \mathrm{~S}: 545.1570[\mathrm{M}+\mathrm{Na}]^{+}$. 


\section{5-Amino-6-[2-[2-(2-iodoxyethoxy)-ethoxy]-ethoxy]-ethoxy-2-methyl-1H-isoindole-}

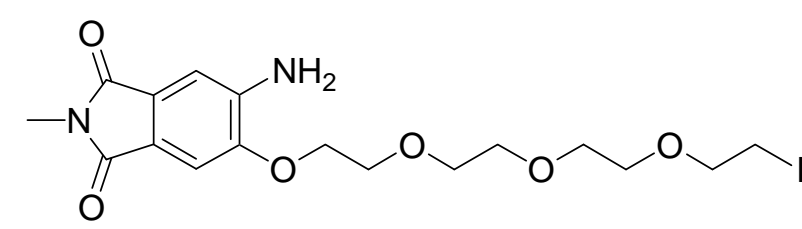

1,3(2H)-dion (102): In the flask equipped with a reflux condenser, compound $\mathbf{1 0 0}(0.10$ $\mathrm{g}, 0.19 \mathrm{mmol})$ was dissolved in acetone $(5$ $\mathrm{mL})$. Then $\mathrm{NaI}(86 \mathrm{mg}, 0.58 \mathrm{mmol})$ was added. The reaction mixture was refluxed overnight, the solvent removed in vacuo, the residue was diluted with water $(10 \mathrm{~mL})$ and $\mathrm{CH}_{2} \mathrm{Cl}_{2}(10 \mathrm{~mL})$. The aqueous layer was extracted with $\mathrm{CH}_{2} \mathrm{Cl}_{2}(2 \times 20 \mathrm{~mL})$. The combined organic solutions were washed with water $(25 \mathrm{~mL})$ and dried. The solvent was removed in vacuo, the residue was purified by chromatography on silica gel (15 g) with $\mathrm{CH}_{2} \mathrm{Cl}_{2} / \mathrm{MeCN}$ mixture (4:1) as an eluent, and the title compound was isolated as a yellow-brown oil (90 mg, 99\%). HPLC: 70/30 $\rightarrow$ 20/80 $(\mathrm{A} / \mathrm{B})$ in $25 \mathrm{~min}, 1 \mathrm{~mL} / \mathrm{min}, 25^{\circ} \mathrm{C}, t_{\mathrm{R}}=14.0 \mathrm{~min}$, detection at $254 \mathrm{~nm} .{ }^{1} \mathrm{H} \mathrm{NMR}(300 \mathrm{MHz}): \delta$ $=3.07(\mathrm{~s}, 3 \mathrm{H}), 3.23\left(\mathrm{t},{ }^{3} J=6.9,2 \mathrm{H}, \mathrm{CH}_{2} \mathrm{I}\right), 3.68\left(\mathrm{~m}, 10 \mathrm{H}, 5 \times \mathrm{CH}_{2}\right), 3.86\left(\mathrm{~m}, 2 \mathrm{H}, \mathrm{CH}_{2}\right), 4.22$ $\left(\mathrm{m}, 2 \mathrm{H}, \mathrm{CH}_{2}\right), 7.02(\mathrm{~s}, 1 \mathrm{H}), 7.16(\mathrm{~s}, 1 \mathrm{H}) \mathrm{ppm} .{ }^{13} \mathrm{C} \mathrm{NMR}(75.5 \mathrm{MHz}): \delta=2.9\left(\mathrm{CH}_{2} \mathrm{I}\right), 23.7$ $\left(\mathrm{CH}_{3} \mathrm{~N}\right), 68.8\left(\mathrm{CH}_{2}\right), 69.4\left(\mathrm{CH}_{2}\right), 70.2\left(\mathrm{CH}_{2}\right), 70.6\left(\mathrm{CH}_{2}\right), 70.7\left(\mathrm{CH}_{2}\right), 70.8\left(\mathrm{CH}_{2}\right), 71.9\left(\mathrm{CH}_{2}\right)$, $106.5(\mathrm{CH}), 108.0(\mathrm{CH}), 121.7$ (C), 127.1 (C), 142.5 (C), 149.3 (C), 169.0 (CO), 169.1 (CO) ppm. ESI-MS, negative mode, m/z (rel. int., \%): 477 (100) $[\mathrm{M}-\mathrm{H}]^{-}$, positive mode, m/z (rel. int., \%): 501 (100) $[\mathrm{M}+\mathrm{Na}]^{+}, 979(20)[2 \mathrm{M}+\mathrm{Na}]^{+}$. HR-MS (ESI) negative mode: found: 477.0520; calcd. for $\mathrm{C}_{17} \mathrm{H}_{23} \mathrm{IN}_{2} \mathrm{O}_{6}: 477.0523[\mathrm{M}-\mathrm{H}]^{-}$, positive mode: found: 501.0502; calcd. for $\mathrm{C}_{17} \mathrm{H}_{23} \mathrm{IN}_{2} \mathrm{O}_{6}: 501.0499[\mathrm{M}+\mathrm{Na}]^{+}$.

Crown ether 92: Method 2 (see Method 1 on page 153): To a solution of compound 102 (60<smiles></smiles>
$\mathrm{mg}, 0.13 \mathrm{mmol})$ in dry DMF $(2 \mathrm{~mL})$ in a dry Schlenk-flask equipped with a septum, $\mathrm{NaI}(38 \mathrm{mg}, 0.25 \mathrm{mmol})$ and DIPEA (66 $\mu \mathrm{L}, \mathrm{d}=0.75,0.39 \mathrm{mmol}$ ) were added at room temperature, and the reaction mixture was heated at $90{ }^{\circ} \mathrm{C}$ for 1 day. All volatile materials were evaporated in vacuo, and the residue was diluted with $\mathrm{CH}_{2} \mathrm{Cl}_{2}(10 \mathrm{~mL})$. The organic solution was washed with water $(10 \mathrm{~mL})$, dried and evaporated. The residue was purified by chromatography on silica gel (30 g) with $\mathrm{CH}_{2} \mathrm{Cl}_{2} / \mathrm{MeCN}$ mixture (1:8) as an eluent, and the title compound was isolated as a yellow solid (18 mg, 40\%). The NMR and mass spectra were reported above. 
Crown ether 92-Et: A mixture of compound $92(17 \mathrm{mg}, 0.05 \mathrm{mmol})$ and $\mathrm{Et}_{2} \mathrm{SO}_{4}(0.2 \mathrm{~mL}, \mathrm{~d}=$ $1.18,1.5 \mathrm{mmol}$ ) was heated at $140{ }^{\circ} \mathrm{C}$ for $1 \mathrm{~h}$ under anhydrous conditions. After cooling to<smiles>CCN1CCOCCOCCOCCOc2cc3c(cc21)C(=O)N(C)C3=O</smiles>
room temperature, the mixture was diluted with ethanol $(5 \mathrm{~mL})$ and poured into ice-cold water $(10 \mathrm{~mL})$. It was strirred for $2 \mathrm{~h}$.

The aqueous solution was extracted with $\mathrm{CH}_{2} \mathrm{Cl}_{2}(2 \times 20 \mathrm{~mL})$. The combined organic solutions were dried. The solvent was removed in vacuo, the residue was purified by chromatography on silica gel (30 g) with $\mathrm{CH}_{2} \mathrm{Cl}_{2}$ / $\mathrm{MeOH}$ mixture (25:1) as an eluent, and the title compound was isolated as a yellow oil (15 mg, 81\%). HPLC: $70 / 30 \rightarrow 20 / 80(\mathrm{~A} / \mathrm{B})$ in $25 \mathrm{~min}, 1 \mathrm{~mL} / \mathrm{min}, 25^{\circ} \mathrm{C}, t_{\mathrm{R}}=4.6 \mathrm{~min}$, detection at $254 \mathrm{~nm} .{ }^{1} \mathrm{H}$ NMR (300 MHz): $\delta=1.13\left(\mathrm{t},{ }^{3} J=7.2,3 \mathrm{H}, \mathrm{CH}_{2} \underline{\mathrm{CH}}_{3}\right), 3.08(\mathrm{~s}, 3 \mathrm{H}), 3.33$ (q, ${ }^{3} J$ $\left.=7.2,2 \mathrm{H}, \underline{\mathrm{CH}_{2}} \mathrm{CH}_{3}\right), 3.39\left(\mathrm{t},{ }^{3} \mathrm{~J}=7.8,2 \mathrm{H}, \mathrm{CH}_{2} \mathrm{~N}\right), 3.66\left(\mathrm{~m}, 8 \mathrm{H}, 4 \times \mathrm{CH}_{2}\right), 3.82\left(\mathrm{t},{ }^{3} \mathrm{~J}=7.2,2\right.$ $\left.\mathrm{H}, \mathrm{CH}_{2}\right), 3.92\left(\mathrm{~m}, 2 \mathrm{H}, \mathrm{CH}_{2}\right), 4.21\left(\mathrm{~m}, 2 \mathrm{H}, \mathrm{CH}_{2}\right), 7.27$ (s, $\left.1 \mathrm{H}\right), 7.30$ (s, $\left.1 \mathrm{H}\right)$ ppm. ${ }^{13} \mathrm{C} \mathrm{NMR}$ (75.5 MHz): $\delta=12.4\left(\mathrm{CH}_{2} \underline{\mathrm{CH}_{3}}\right), 23.8\left(\mathrm{CH}_{3} \mathrm{~N}\right), 46.3\left(\underline{\mathrm{CH}_{2}} \mathrm{CH}_{3}\right), 52.5\left(\mathrm{CH}_{2} \underline{\mathrm{CH}}_{2} \mathrm{~N}\right), 68.7\left(\mathrm{CH}_{2}\right)$, $69.2\left(2 \times \mathrm{CH}_{2}\right), 69.9\left(\mathrm{CH}_{2}\right), 70.1\left(\mathrm{CH}_{2}\right), 70.2\left(\mathrm{CH}_{2}\right), 70.7\left(\mathrm{CH}_{2}\right), 106.3(\mathrm{CH}), 111.8(\mathrm{CH})$, 123.7 (C), 125.8 (C), 145.5 (C), 154.8 (C), 169.0 (CO), 169.2 (CO) ppm. ESI-MS, positive mode, m/z (rel. int., \%): 379 (20) $[\mathrm{M}+\mathrm{H}]^{+}, 401$ (100) $[\mathrm{M}+\mathrm{Na}]^{+}$. HR-MS (ESI, positive mode): found: 379.1862, 401.1685; calcd. for $\mathrm{C}_{19} \mathrm{H}_{26} \mathrm{~N}_{2} \mathrm{O}_{6}: 379.1869[\mathrm{M}+\mathrm{H}]^{+}, 401.1689[\mathrm{M}$ $+\mathrm{Na}]^{+}$.

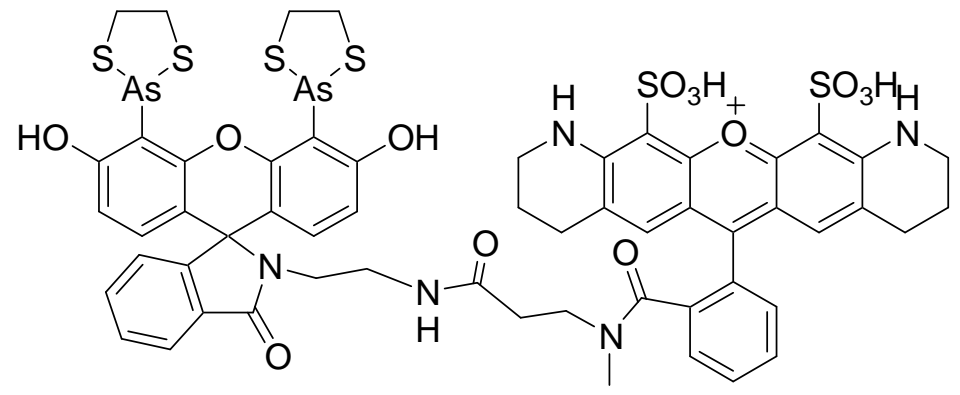

SplAsH-RhS-OF: According to the GP6, compound 118-Me $(5.5 \mathrm{mg}, 7.3 \mu \mathrm{mol})$ and compound 105a (15 mg, $21 \mu \mathrm{mol})$ gave the title compound as a pink solid $(3.1 \mathrm{mg}, 32 \%)$. Preparative HPLC: $60 / 40 \rightarrow 0 / 100(\mathrm{~A} / \mathrm{B})$ in $25 \mathrm{~min}, 4 \mathrm{~mL} / \mathrm{min}, 7^{\circ} \mathrm{C}, t_{\mathrm{R}}=10.1 \mathrm{~min}$, detection at $530 \mathrm{~nm}$. Analytical HPLC: $60 / 40 \rightarrow 0 / 100(\mathrm{~A} / \mathrm{B})$ in $25 \mathrm{~min}, 1 \mathrm{~mL} / \mathrm{min}, 7^{\circ} \mathrm{C}, t_{\mathrm{R}}=12.8 \mathrm{~min}$, detection at $530 \mathrm{~nm}$. ESI-MS, positive mode, m/z (rel. int., \%): $1388(100)[\mathrm{M}+2 \mathrm{Na}-\mathrm{H}]^{+}$. HR-MS (ESI, positive mode): found: 1387.9951; calcd. for $\mathrm{C}_{56} \mathrm{H}_{51} \mathrm{As}_{2} \mathrm{~N}_{5} \mathrm{O}_{13} \mathrm{~S}_{6}: 1387.9957$ [M $+2 \mathrm{Na}-\mathrm{H}]^{+}$ 


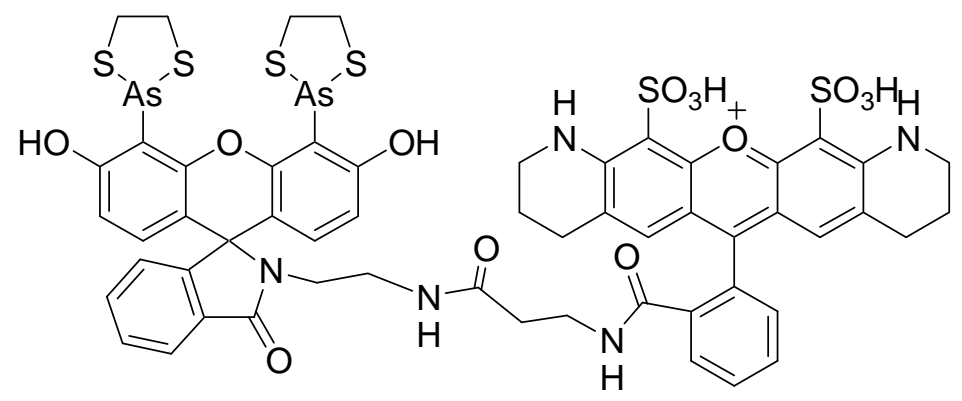

SplAsH-RhS-CF: According to the GP6, compound 118-H (6.0 $\mathrm{mg}, 8.1 \mu \mathrm{mol})$ and compound 105a $(15 \mathrm{mg}, 21 \mu \mathrm{mol})$ gave the title compound as a light rosy solid $(2.0 \mathrm{mg}$, 19\%). Preparative HPLC: $60 / 40 \rightarrow 0 / 100(\mathrm{~A} / \mathrm{B})$ in $25 \mathrm{~min}, 4 \mathrm{~mL} / \mathrm{min}, 7{ }^{\circ} \mathrm{C}, t_{\mathrm{R}}=9.7 \mathrm{~min}$, detection at $530 \mathrm{~nm}$. Analytical HPLC: $80 / 20 \rightarrow 50 / 50(\mathrm{~A} / \mathrm{B})$ in $25 \mathrm{~min}, 1 \mathrm{~mL} / \mathrm{min}, 25{ }^{\circ} \mathrm{C}, t_{\mathrm{R}}$ $=10.7 \mathrm{~min}$, detection at $254 \mathrm{~nm}$. ESI-MS, negative mode, m/z (rel. int., \%): 1350 (100) [M + $\mathrm{Na}-2 \mathrm{H}]^{-}$, positive mode, m/z (rel. int., \%): 1390 (100) $[\mathrm{M}+\mathrm{Na}+\mathrm{K}-\mathrm{H}]^{+}$. HR-MS (ESI, negative mode): found: 663.4969 ; calcd. for $\mathrm{C}_{55} \mathrm{H}_{49} \mathrm{As}_{2} \mathrm{~N}_{5} \mathrm{O}_{13} \mathrm{~S}_{6}: 663.4963[\mathrm{M}-2 \mathrm{H}]^{2-}$.

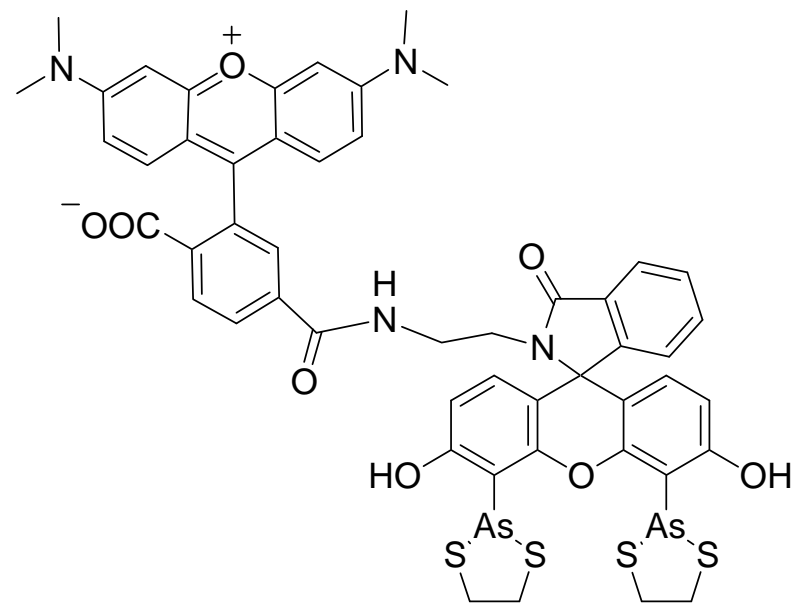

SpIAsH-TAMRA: To a solution of the NHS-ester of carboxy TMR-rhodamine (119) (2.5 $\mathrm{mg}, 4.7 \mu \mathrm{mol})$ and DIPEA $(10 \mu \mathrm{L}, \mathrm{d}=0.72,57 \mu \mathrm{mol})$ in a dry DMF $(0.75 \mathrm{~mL})$, the solution of compound 105a $(10 \mathrm{mg}, 14 \mu \mathrm{mol})$ in $\mathrm{CH}_{2} \mathrm{Cl}_{2}(0.5 \mathrm{~mL})$ was added via a syringe, and the reaction mixture was stirred at room temperature for $6 \mathrm{~h}$. Then all volatile materials were removed in vacuo (without heating). The residue was purified by the column reversed-phase chromatography and gradient eluting first with $\mathrm{MeCN} / \mathrm{H}_{2} \mathrm{O}$ mixture $(4: 1 \rightarrow 10: 1)$ and then with $\mathrm{MeOH}$. The residue after freeze-drying was purified by preparative HPLC: 50/50 $\rightarrow$ 0/100 (A/B) in $25 \mathrm{~min}, 16 \mathrm{~mL} / \mathrm{min}, 25^{\circ} \mathrm{C}, t_{\mathrm{R}}=11.8 \mathrm{~min}$, detection at $547 \mathrm{~nm}$. The solvents were removed by the freeze-drying. The title compound was isolated as a red solid $(3.3 \mathrm{mg}$, $62 \%$ ). Analytical HPLC: $50 / 50 \rightarrow 0 / 100(\mathrm{~A} / \mathrm{B})$ in $25 \mathrm{~min}, 1 \mathrm{~mL} / \mathrm{min}, 25{ }^{\circ} \mathrm{C}, t_{\mathrm{R}}=13.7 \mathrm{~min}$, 
detection at $254 \mathrm{~nm}$. ESI-MS, negative mode, m/z (rel. int., \%): 1117 (100) [M - H] $]^{-}$, positive mode, m/z (rel. int., \%): $1119(100)[\mathrm{M}+\mathrm{H}]^{+}, 1141(100)[\mathrm{M}+\mathrm{Na}]^{+}$. HR-MS (ESI, negative mode): found: 1119.0540; calcd. for $\mathrm{C}_{51} \mathrm{H}_{44} \mathrm{As}_{2} \mathrm{~N}_{4} \mathrm{O}_{8} \mathrm{~S}_{4}: 1119.0552[\mathrm{M}+\mathrm{H}]^{+}$. 


\section{Summary}

In the present work, the crown ether-containing rhodol dyes (compounds Ia, Ib, Ic, 70) have been synthesized, and their spectral as well as their calcium-indicator properties have been investigated in methanolic and aqueous solutions. As a part of this study, the feasibility of the synthesis of the target sensors Ia-c was evaluated by performing the synthesis of some simplified model compounds. A total synthesis of the colorless rhodole spiroamide $40-\mathrm{H}$ (model compound for the target Ia-c, IIa,b) was developed, and the photoactivation of the latter by irradiation with UV light was tested. This compound was found to be photosensitive (under irradiation at $366 \mathrm{~nm}$ ), and it gave a fluorescent species in toluene solution.

The excitation and emission maxima of all prepared dyes are above $450 \mathrm{~nm}\left(\lambda_{a b s}^{\max }=513-\right.$ $\left.542 \mathrm{~nm}\left(\varepsilon=(2.2-5.2) \times 10^{4} \mathrm{M}^{-1} \mathrm{~cm}^{-1}\right), \lambda_{f l}^{\max }=539-558 \mathrm{~nm}\right)$, and fulfil the required spectral criteria for calcium indicators (see section 1 of Chapter 1). The "brightness" $(\varepsilon \bullet \Phi)$ varies from 3500 (for compound Ib) to $41600 \mathrm{M}^{-1} * \mathrm{~cm}^{-1}$ (for compound 70). The absorption and emission spectra of all prepared rhodols do not depend on the $\mathrm{pH}$ (in the range of intracellular $\mathrm{pH}$ values, i. e. $\mathrm{pH}=6-8)$.

A junction of the crown ether with an aromatic ring in the xanthene moiety through only one heteroatom (nitrogen) leads to a lower response upon the presence of calcium in the medium than in the case of the fusion of the crown ether with the xanthene by two heteroatoms. This conclusion was confirmed by the absence of changes in the fluorescence signal of aqueous solutions of compound $\mathbf{7 0}$ in the absence and presence of calcium ions.

Compound Ia with an NH group in the crown-ether ring was not sensitive to calcium at any $\mathrm{pH}$ value in aqueous solution, and the same feature was observed for compound Ic with a $\mathrm{CH}_{2} \mathrm{COOH}$ substituent at the nitrogen atom of the crown-ether ring. However, the fluorescence of compound Ib with an ethyl group at the nitrogen atom of the crown-ether ring strongly increased in the presence of calcium cations in the millimolar range $(1-126 \mathrm{mM})$. The observed dependence failed to fit any of the most obvious models, such as the formation of $1: 1$ and $2: 1$ complexes between the indicator and calcium. Therefore, the stability constant of the complex cannot be calculated.

Key precursors (compounds 92, 92-Et) to the target compounds IIa,b were prepared. It was established, that the most efficient route to compound 92 is by twofold alkylation of 4amino-5-hydroxy- $N$-methylphthalimide 90 with the diiodide 91. In spite of the low yield of compound $92(14 \%)$, the shorter sequence is preferable, because the longer route with 
consecutive alkylation of the nitrophenolic salt 89 by the iodide $\mathbf{9 6}$, deprotection, tosylation, reduction, nucleophilic substitution and cyclization provides the same overall yield (14\%).

Several fluorescent labels for the tetracysteine tags in proteins with the water-soluble orange-emitting dye Rhodamine S (SplAsH-RhS-OF), with the same dye in the masked spiroamide form (SplAsH-RhS-CF) and with TMR-dye (SplAsH-TAMRA) were prepared, and their binding and staining properties were investigated. Due to the extreme instability of compounds SplAsH-RhS-OF and SplAsH-RhS-CF, a special HPLC procedure for their isolation was elaborated. The binding propeties were evaluated in competitive mode with FlAsH (Fluorescein Arsenic Hairpin binder) fluorimetrically. Comparison of the fluorescence intensity in a control experiment (FlAsH + peptide with the tetracysteine fragment) and for the same mixture with the added compound SplAsH-RhS-OF, confirmed the binding ability of the SplAsH derivates. The second series of experiments with the non-fluorescent compound SplAsH-RhS-CF, instead of the orange-emitting SplAsH-RhS-OF, provided information on the binding ability of FlAsH in a competitive assay: the amount of unbound (non-fluorescent) FlAsH was 7 times higher than that of the bound (fluorescent) form. This result confirmed the good binding properties of the SplAsH derivates (SplAsH-RhS-OF, SplAsH-RhS-CF).

The third series of experiments dealt with the labelling of proteins with some SplAsHcontaining compounds. The proteins differ from each other by the number of tetracysteine moieties ( 2 or 3 ) and the "degrees of freedom" (free protein or the protein incorporated into cell structures). The labels could be introduced into organells inside live cells or into isolated cell structures. In all experiments with isolated proteins, products of their binding with SplAsH-RhS-OF could be detected. However, the introduction of the SplAsH-RhS-OF label into proteins in live cells was not selective. This conclusion was made by studying the cell images in the confocal microscope. The entire cells were colored, without any preference for the structures with tetracysteine tags.

Further, labelling of mitochondria with SpIAsH-TAMRA was attempted. It is wellknown, that rhodamines are preliminarily localized in mitochondria. ${ }^{[141]}$ Therefore, this property was expected to help in introducing the SplAsH labels into mitochondria. However, using SplAsH-TAMRA, the same picture as in the case of SplAsH-RhS-OF was observed (the entire cells were colored inside). Therefore, the mitochondria could not be labelled selectively by the marker SplAsH-TAMRA. 

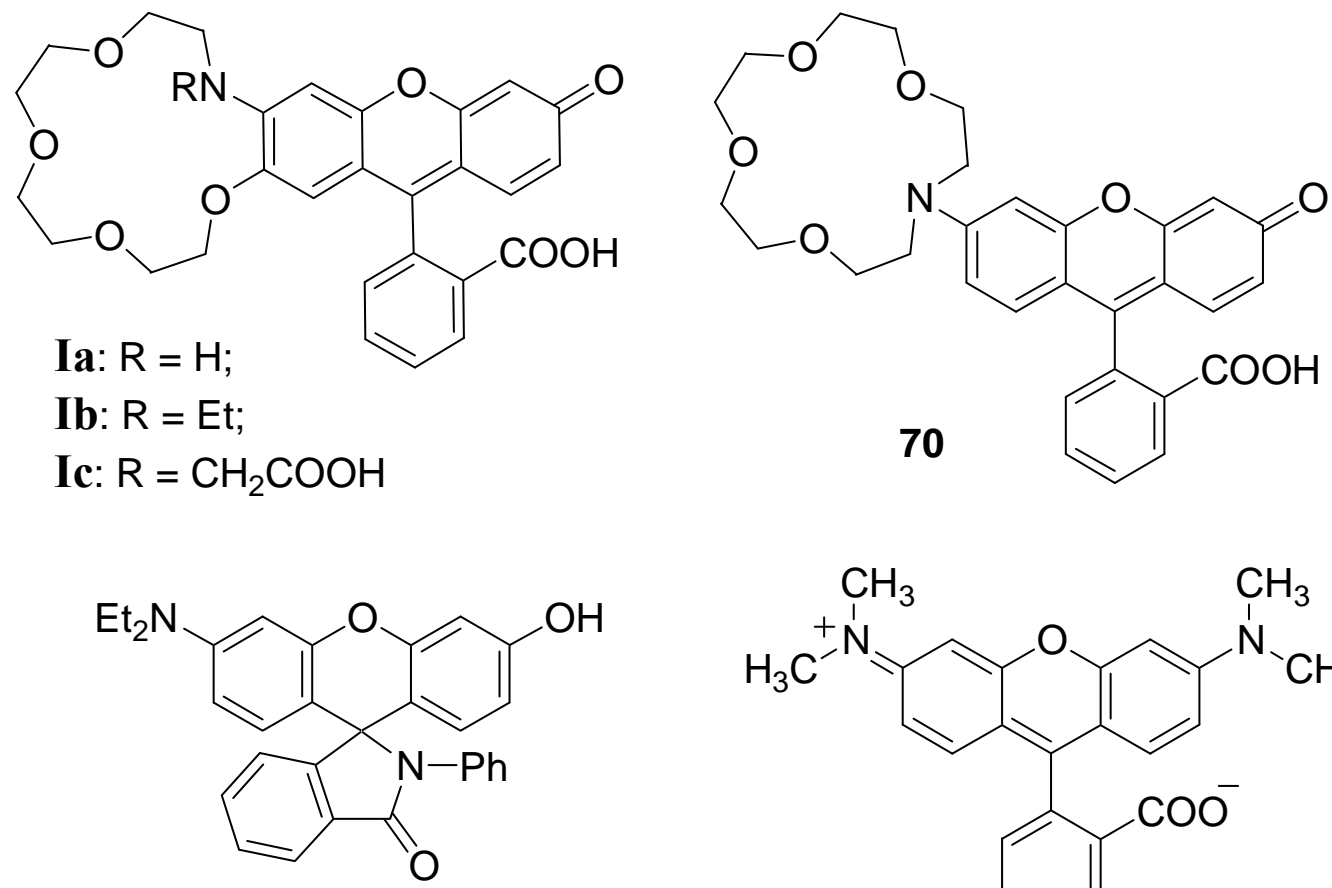

40- $\mathrm{H}$
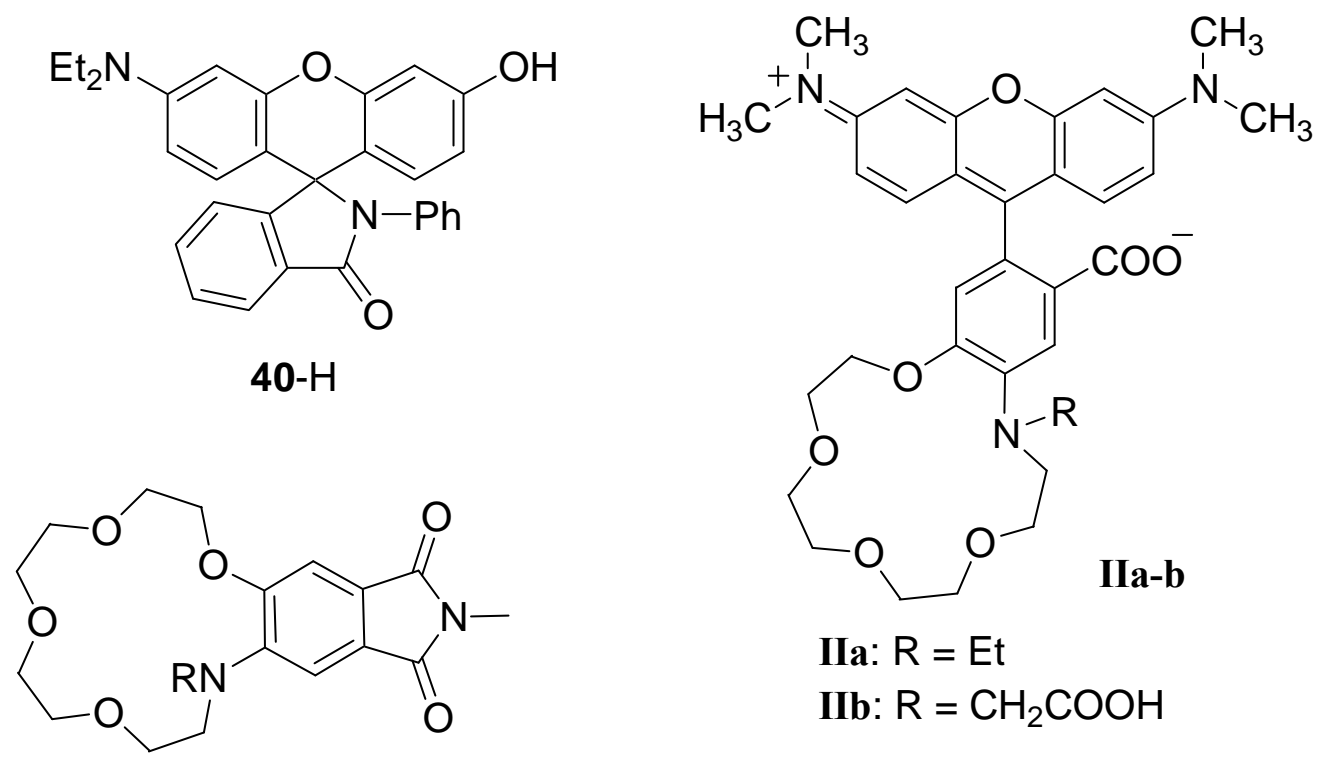

IIa: $\mathrm{R}=\mathrm{Et}$

IIb: $\mathrm{R}=\mathrm{CH}_{2} \mathrm{COOH}$

92: $\quad R=H$

92-Et: $R=E t$<smiles>ICCOCCOCCOCCI</smiles><smiles>CN1C(=O)c2cc(N)c(O)cc2C1=O</smiles><smiles>[R6]c1cc2c(cc1[N+](=O)[O-])C(=O)N(C)C2=O</smiles>

91<smiles>[OH2+]CCOCCOCCOCCI</smiles>
96<smiles></smiles> 


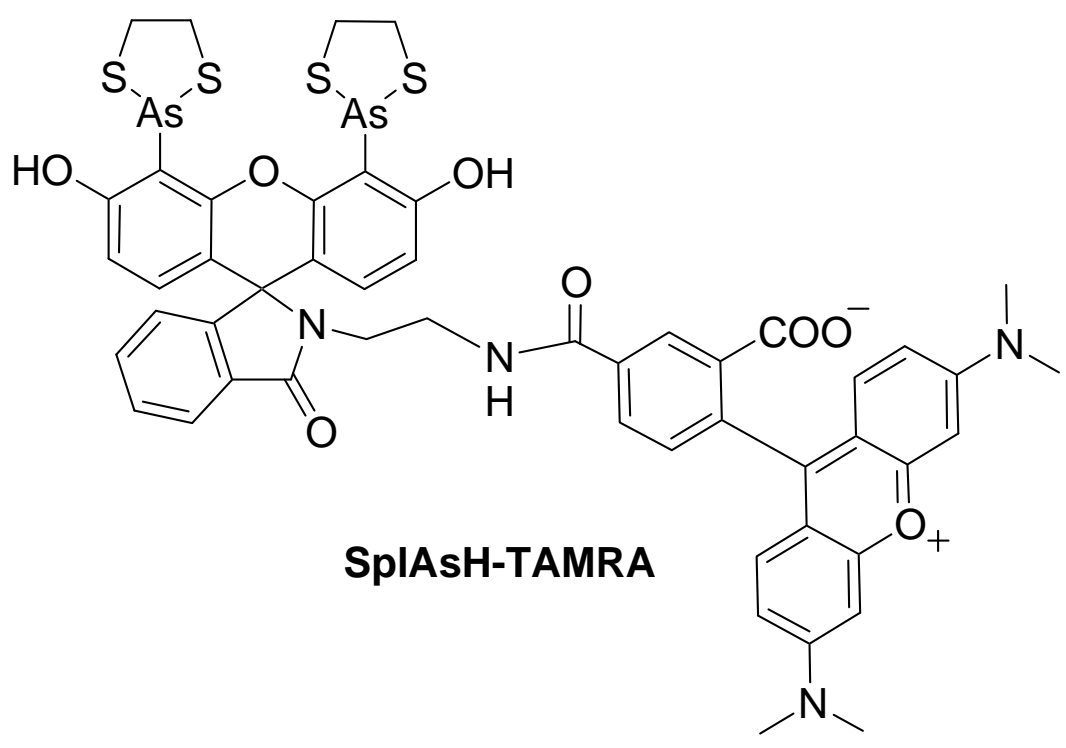




\section{References}

[1] G. Clark, F. H. Kasten in History of Staining (Eds.: H. J. Conn, L. Arnold), $3^{\text {rd }}$ Edition, Williams \& Wilkins, Baltimore, 1983, pp. 35-43.

[2] M. Zimmer, Chem. Rev. 2002, 102, 759-781.

[3] B. A. Griffin, S. R. Adams, R. Y. Tsien, Science 1998, 281, 269-272.

[4] F. Prendergast, K. Mann, Biochemistry 1978, 17, 3448-3453.

[5] J. Fölling, V. Belov, R. Kunetsky, R. Medda, A. Schönle, A. Egner, C. Eggeling, M. Bossi, S. W. Hell, Angew. Chem. 2007, 119, 6382-6386; Angew. Chem. Int. Ed. 2007, 46, 6266-6270.

[6] V. N. Belov, C. A. Wurm, V. P. Boyarskiy, S. Jakobs, S. W. Hell, Angew. Chem. 2010, 122, 3598-3602; Angew. Chem. Int. Ed. 2010, 49, 3520-3523.

[7] A. K. Bhunia, S. C. Miller, ChemBioChem 2007, 8, 1642-1645.

[8] V. P. Boyarskiy, V. N. Belov, R. Medda, B. Hein, M. Bossi, S. W. Hell, Chem. Eur. J. 2008, 14, 1784-1792.

[9] R. P. Haugland in The Handbook: A Guide to Fluorescent Probes and Labeling Technologies Microscopy (Ed.: M. T. Z. Spence), $10^{\text {th }}$ Edition, Invitrogen Corp., USA, 2006, pp. 894-895.

[10] For a review on the history of fluorescence, see: Molecular Fluorescence (Ed.: B. Valeur), Wiley-VCH, Weinheim, 2002, 3-8.

[11] Stockes G. G., Phil. Trans. 1853, 143, 385-396.

[12] a) A. Köhler, H. Siedentopf, "Dunkelfeldbeleuchtung im ultravioletten Licht", 1908 (Quoted by W. Loos, Zeiss Nachr. 1939, 2. Folge, 319-326); b) for a review on the history of fluorescence microscopy see: F. W. D. Rost in Fluorescence Microscopy, Cambridge University Press, Cambridge, 1995, Vol. II, pp. 183-196.

[13] E. Abbe, Arch. Mikr. Anat. 1873, 9, 413-468.

[14] S. W. Hell, J. Wichmann, Opt. Lett. 1994, 19, 780-782.

[15] S. W. Hell, Phys. Lett. A 2004, 326, 140-145.

[16] S. W. Hell, Nat. Methods 2009, 6(1), 24-32.

[17] E. Betzig, G. H. Patterson, R. Sougrat, O. W. Lindwasser, S. Olenych, J. S. Bonifacino, M. W. Davidson, J. Lippincott-Schwartz, H. F. Hess, Science 2006, 313, 1642-1645.

[18] A. C. Meyer, T. Frank, D. Khimich, G. Hoch, D. Riedel, N. M. Chapochnikov, Y. M. Yarin, B. Harke, S. W. Hell, A. Egner, T. Moser, Nat. Neurosci. 2009, 12(4), 444-453. 
[19] M. J. Berridge, M. D. Bootman, H. L. Roderick, Nature Molecular Cell Biology 2003, 4, $517-529$.

[20] L. M. Popescu, I. Diculescu, U. Zelck, N. Ionescu, Cell Tiss. Res. 1974, 154, 357-378.

[21] S. L. Howell, W. Montague, M. Tyhurst, J. Cell Sci. 1975, 19, 395-409.

[22] The higher $K_{\mathrm{D}}$ value corresponds to a lower affinity of an indicator molecule for the metal ion.

[23] B. Huang, Curr. Opin. Chem. Biol. 2010, 14, 10-14.

[24] H. Diehl, J. L. Ellingboe, Anal. Chem. 1956, 28, 882-884.

[25] D. H. Wilkins, Talanta 1960, 4, 182-184.

[26] G. M. Huitink, Proc. Ind. Acad. Sci. 1973, 82, 161-166.

[27] D. W. Fink, W. R. Koehler, Anal. Chem. 1970, 42, 990-993.

[28] G. M. Huitink, H. Diehl, Talanta 1974, 21, 1193-1202.

[29] V. Michaylova, P. Ilkova, Anal. Chem. Acta 1971, 53, 194-198.

[30] V. I. Kuznetsov, Doklady Akad. Nauk USSR 1941, 31, 895.

[31] J. E. Brown, L. B. Cohen, P. De Weer, L. H. Pinto, W. N. Ross, B. M. Salzberg, Biophys. J. 1975, 15, 1155-1160.

[32] R. Y. Tsien, Biochemistry 1980, 19, 2396-2404.

[33] M. V. Thomas, Biophys. J. 1979, 25, 541-548.

[34] R. Y. Tsien, Nature 1981, 290, 527-528.

[35] a) R. Y. Tsien, G. Grynkiewicz (The Regents of the University of California), US Patent 648649 (07.09.1984); b) G. Grynkiewicz, M. Poenie, R. Y. Tsien, J. Biol. Chem. 1985, 260 (6), 3440-3450.

[36] R. M. DeMarinis, H. E. Katerinopoulos, K. A. Muirhead (SmithKline Beckman Corporation), US Patent 4,849,362 (18.07.1989).

[37] A. L. Fogelson, R. S. Zucker, Biophys. J. 1985, 48, 1003-1017.

[38] a) E. F. Etter, M. A. Kuhn, F. S. Fay, J. Biol. Chem. 1994, 269 (13), 10141-10149; b) M.

F. Poenie, A. Minta (Texas Fluorescence Laboratories, Inc.), US Patent 5,576,433 (19.11.1996).

[39] a) R. E. London, C. K. Rhee, E. Murphy, S. Gabel, L. A. Levy, Am. J. Physiol. 1994, 266, C1313-C1322; b) R. E. London, L. A. Levy, E. Murphy (Department of Health and Human Services, USA), US Patent 5,516,911 (14.05.1996).

[40] K. R. Gee, E. A. Archer, L. A. Lapham, M. E. Leonard, Z.-L. Zhou, J. Bingham, Z. Diwu, Bioorg. Med. Chem. Lett. 2000, 10, 1515-1518. 
[41] R. M. Sanchez-Martin, M. Cuttle, S. Mittoo, M. Bradley, Angew. Chem. Int. Ed. 2006, $45,5472-5474$.

[42] M. Bannwarth, I. R. Corrêa Jr., M. Sztretye, S. Pouvreau, C. Fellay, A. Aebischer, L. Royer, E. Ríos, K. Johnsson, ACS Chem. Biol. 2009, 4(3), 179-190.

[43] For a review on the working principles of the SNAP-tag technology, see: A. Keppler, S. Gendreizig, T. Gronemeyer, H. Pick, H. Vogel, K. Johnsson, Nat. Biotechnol. 2003, 21, 8689.

[44] a) R. Y. Tsien, M. Akwasi (The Regents of the University of California), EP 0314480 A2 (03.05.1989); b) M. Akwasi, J. P. Y. Kao, R. Y. Tsien, J. Biol. Chem. 1989, 264 (14), $8171-8178$.

[45] S. R. Adams, J. P. Y. Kao, G. Grynkiewicz, A. Minta, R. Y. Tsien, J. Am. Chem. Soc. 1988, 110, 3112-3220.

[46] J. H. Kaplan, G. C. R. Ellis-Davies, Proc. Natl. Acad. Sci. USA, 1988, 85, 6571-6575.

[47] K. R. Gee, K. A. Brown, W.-N. U. Chen, J. Bishop-Steward, D. Gray, I. Johnson, Cell Calcium 2000, 27(2), 97-106.

[48] V. V. Martin, M. Beierlein, J. L. Morgan, A. Rothe, K. R. Gee, Cell Calcium 2004, 36, $509-514$.

[49] A. Minta, R. Escamilla, E. A. Bates, US Patent Application Publication US 2005/0233467 A1 (20.10.2005).

[50] J. Han, J.-L. Li, H.-Z. He, X.-L. Wu, Z. Shi, Chem. J. Chin. Univ. 2008, 29(10), 20032006.

[51] Z. Diwu, J. He, J. Liao, US Patent Application Publication US 2008/0254498 A1 (16.10.2008).

[52] S. Gaillard, A. Yakovlev, C. Luccardini, M. Oheim, A. Feltz, J.-M. Mallet, Org. Lett. 2007, 9(14), 2629-2632.

[53] H. Iatridou, E. Foukaraki, M. A. Kuhn, E. M. Marcus, R. P. Haugland, H. E. Katerinopoulos, Cell Calcium 1994, 15, 190-198.

[54] K. Takesako, K. Sasamoto, Y. Ohkura, K. Hirose, M. Iino, Anal. Comm. 1997, 34, 391394.

[55] F. Liepouri, E. Foukaraki, T. G. Deligeorgiev, H. E. Katerinopolus, Cell Calcium 2001, $30(5), 331-335$.

[56] F. Liepouri, T. G. Deligeorgiev, Z. Veneti, C. Savakis, H. E. Katerinopoulos, Cell Calcium 2002, 31(5), 221-227. 
[57] H. Komatsu, T. Miki, D. Citterio, T. Kubota, Y. Shindo, Y. Kitamura, K. Oka, K. Suzuki, J. Am. Chem. Soc. 2005, 127(31), 10798-10799.

[58] Q. P. Lloyd, M. A. Kuhn, C. V. Gay, J. Biol. Chem. 1995, 270 (38), 22445-22451.

[59] A. Prasanna de Silva, H. Q. N. Gunaratne, J. Chem. Soc., Chem. Commun. 1990, 186188.

[60] D. C. Magri, J. F. Callan, A. Prasanna de Silva, D. B. Fox, N. D. McClenaghan, K. R. A. Samankumara Sandanayake, J. Fluoresc. 2005, 15(5), 769-775.

[61] A. Prasanna de Silva, H. Q. N. Gunaratne, A. T. M. Kane, G. E. M. Maguire, Chem. Lett. 1995, 2, 125-126.

[62] a) E. U. Akkaya, S. Turkyilmaz, Tetrahedron Lett. 1997, 38 (25), 4513-4516; b) E. U. Akkaya, NATO ASI Series, Series C: Mathematical and Physical Science 1997, 492, 177188.

[63] B. Ozmen, E. U. Akkaya, Tetrahedron Lett. 2000, 41, 9185-9188.

[64] G. A. Smith, J. C. Metcalfe, S. D. Clarke, J. Chem. Soc. Perkin Trans. 2 1993, 11951204.

[65] E. Cielen, A. Stobiecka, A. Tahri, G. J. Hoornaert, F. C. De Schryver, J. Gallay, M. Vincent, N. Boens, J. Chem. Soc. Perkin Trans. 2 2002, 1197-1206.

[66] N. Avcibaşi, M. Smet, B. Metten, W. Dehaen, F. C. De Schryver, G. Bultynck, G. Callewaert, H. De Smedt, L. Missiaen, N. Boens, Int. J. Photoenergy 2004, 6, 159-167.

[67] N. Basaric, M. Baruah, W. Qin, B. Metten, M. Smet, W. Dehaen, N. Boens, Org. Biomol. Chem. 2005, 3, 2755-2761.

[68] N. Boens, N. Avcibaşi, S. S. Samanta, A. Kilonda, G. J. Hoornaert, E. Van der Eycken, Tetrahedron 2006, 62, 684-690.

[69] H. Nishida, Y. Katayama, H. Katsuki, H. Nakamura, M. Takagi, K. Ueno, Chem. Lett. 1982, 1853-1854.

[70] S. Iwata, H. Matsuoka, K. Tanaka, J. Chem. Soc. Perkin Trans. 1 1997, 1357-1360.

[71] a) J.-F. Letard, R. Lapouyade, W. Rettig, Mol. Cryst. Liq. Cryst. 1993, 236, 41-46; b) J.F. Letard, R. Lapouyade, W. Rettig, Pure Appl. Chem. 1993, 65(8), 1705-1712.

[72] S. Delmond, J.-F. Letard, R. Lapouyade, New J. Chem. 1996, 20, 861-869.

[73] a) A. J. Pearson, W. Xiao, J. Org. Chem. 2003, 68(13), 5361-5368; b) A. J. Pearson, J.-J. Hwang, M. E. Ignatov, Tetrahedron Lett. 2001, 42, 3537-3540.

[74] S. Fery-Forgues, M.-T. Le Bris, J.-P. Guette, B. Valeur, J. Phys. Chem. 1988, 92, 62336237.

[75] J. Bourson, B. Valeur, J. Phys. Chem. 1989, 93, 3871-3876. 
[76] D. B. McQueen, K. S. Schanze, J. Am. Chem. Soc. 1991, 113, 6108-6110.

[77] K. Yoshida, T. Mori, S. Watanabe, H. Kawai, T. Nagamura, J. Chem. Soc. Perkin Trans. 2 1999, 393-397.

[78] a) K.-C. Wu, Y.-S. Lin, Y.-S. Yeh, C.-Y. Chen, M. O. Ahmed, P.-T. Chou, Y.-S. Hon, Tetrahedron 2004, 60, 11861-11868; b) K.-C. Wu, Y.-M. Cheng, Y.-S. Lin, Y.-S. Yeh, S.-C. Pu, Y.-H. Hu, J.-K. Yu, C.-Y. Chen, P.-T. Chou, Chem. Phys. Lett. 2004, 384, 203-209.

[79] L.-H. Liu, H. Zhang, A.-F. Li, J.-W. Xie, Y.-B. Jiang, Tetrahedron 2006, 62, 1044110449.

[80] A. J. Pearson, W. Xiao, J. Org. Chem. 2003, 68(13), 5369-5376.

[81] C.-M. Jin, G.-Y. Lu, C.-Z. Zhang, Z.-W. Shi, X.-Z. You, Chin. J. Chem. 2003, 21, 14101413.

[82] a) S. G. Kwon, E. H. Lee, J. S. Kim (Korea Atomic Energy Research Institute; Korea Hydro \& Nuclear Power Co., Ltd.), KR102006039252AA (08.05.2006); b) H.-F. Ji, Y. Yang, X. Xu, G. Brown, Org. Biomol. Chem. 2006, 4, 770-772.

[83] H. Dürr, R. Schwarz, I. Willner, E. Joselevich, Y. Eichen, J. Chem. Soc., Chem. Commun. 1992, 1338-1339.

[84] Y. Kakizawa, T. Akita, H. Nakamura, Chem. Lett. 1993, 1671-1674.

[85] J. Kawakami, Y. Komai, T. Sumori, A. Fukushi, K. Shimozaki, S. Ito, J. Photochem. Photobiol. A 2001, 139, 71-78.

[86] Y. Park, J. Pullen, R. C. Advincula, Polymer Preprints 2008, 49(1), 601-602.

[87] The long-time boiling the methyl ester of the fluorescein with the primary aliphatic amine in DMF: M. Adamczyk, J. Grote, Synth. Commun. 2001, 31, 2681-2690.

[88] S. P. Gromov, S. N. Dmitrieva, A. I. Vedernikov, L. G. Kuz'mina, A. V. Churakov, Y. A. Strelenko, J. A. K. Howard, Eur. J. Org. Chem. 2003, 3189-3199.

[89] A. P. Molchanov, V. V. Razin, V. P. Semenov, Z. A. Shevchenko in Methodical instructions to the practical works in organic synthesis (Eds.: K. A. Ogloblin, R. R. Kostikov), Leningrad State University, Leningrad, 1982, pp. 20-22.

[90] F. W. Gubitz, J. Med. Chem. 1985, 28, 728-733.

[91] T. Cohen, A. G. Dietz, Jr., J. R. Miser, J. Org. Chem. 1977, 42, 2053-2058.

[92] F. Mao, W.-Y. Leung, R. P. Haugland (Molecular Probes, Inc.), WO 99/15517 (23.09.1998).

[93] In the following article it is described the procedure for synthesis of rhodol with the starting material 3-N,N-dimethylaminophenol, instead of 3-N,N-diethylaminophenol 44 which 
we used: R. R. Sauers, S. N. Husain, A. P. Piechowski, G. R. Bird, Dyes Pigments 1987, 8, $35-53$.

[94] For the removal of the methyl protecting group from phenols in the presence of amide group in structure we used the slightly modified procedure from the following article: $\mathrm{F}$. I. Carroll, M. S. Melvin, M. C. Nuckols, S. W. Mascarella, H. A. Navarro, J. B. Thomas, J. Med. Chem. 2006, 49, 1781-1791.

[95] T.-L. Chang, H. C. Cheung, Chem. Phys. Lett., 1990, 173, 343-348.

[96] K. Gee, V. Martin (Molecular Probes, Inc.), WO 2005/016874 A2 (24.02.2005).

[97] M. H. Bromels, C. J. Pedersen (E. I. Du Pont De Nemours and Co., Wilmington, Del., USA), DE 2123256 A1 (23.12.1971).

[98] D. B. Amabilino, P. R. Ashton, C. L. Brown, E. Cordova, L. A. Godínez, T. T. Goodnow, A. E. Kaifer, S. P. Newton, M. Pietraszkiewicz, D. Philp, F. M. Raymo, A. S. Reder, M. T. Rutland, A. M. Z. Slawin, N. Spencer, J. F. Stoddart, D. J. Williams, J. Am. Chem. Soc. 1995, 117, 1271-1293.

[99] R. A. Bartsch, E. Chapoteau, B. P. Czech, J. Krzykawski, A. Kumar, T. W. Robinson, J. Org. Chem. 1993, 58, 4681-4684.

[100] C. H. Heathcock, T. A. Blumenkopf, K. M. Smith, J. Org. Chem. 1989, 54, 1548-1562.

[101] H. Li, Y. Bleriot, C. Chantereau, J.-M. Mallet, M. Sollogoub, Y. Zhang, E. RodriguezGarcia, P. Vogel, J. Jimenez-Barbero, P. Sinaÿ, Org. Biomol. Chem. 2004, 2, 1492-1499.

[102] V. N. Belov, M. L. Bossi, J. Fölling, V. P. Boyarskiy, S. W. Hell, Chem. Eur. J. 2009, $15,10762-10776$.

[103] S. Urgaonkar, J. G. Verkade, Tetrahedron 2004, 60, 11837-11842.

[104] I. T. Harrison, J. Chem. Soc. D 1969, 616a.

[105] P. R. Hammond, J. Photochem., 1979, 10, 467-471.

[106] H. Goldstein, J.-P. Merminod, Helv. Chim. Acta 1952, 35, 1476-1480.

[107] Cited procedure, which was described for the preparation of 2,5-dinitrotoluene from 2methyl-4-nitroaniline, works also in our case: H. J. Page, B. R. Heasman, J. Chem. Soc. 1923, $123,3235-3242$.

[108] In the following article is described the conditions for oxidation 1,2-dimethyl-4methoxybenzene (89) which was successfully applied in our work for oxidation of the compound 94: J. Zoń, P. Miziak, N. Amrhein, R. Gancarz, Chem. Biodivers. 2005, 2, 11871194.

[109] J. C. Cain, J. L. Simonsen, J. Chem. Soc., Trans. 1914, 105, 156-165. 
[110] In the following article is described the conditions for the synthesis of nitro TMRderivative from 4-nitrophthalic anhydride and 3-(N,N-dimethylamino)phenol: J. L. Riggs, R. G. Seiwald, J. H. Burckhalter, C. M. Downs, T. G. Metcalf, Am. J. Pathol. 1958, 34, 10811097.

[111] L. R. Caswell, M. Guevara, L. D. Corley, A. V. Martínez, T. Hollis, K. Largess, D. L. Thornley, Synthesis 1992, 823-825.

[112] The slightly modified procedure from ref. [111] was used.

[113] L. R. Caswell, G. Cavasos, J. Heterocyclic Chem. 1995, 32, 907-914.

[114] a) Compound 91 was prepared from compound $\mathbf{5 9}$ by the nucleophilic substitution of chlorine with iodide as described: B. Jousselme, P. Blanchard, E.. Levillain, J. Delaunay, M. Allain, P. Richomme, D. Rondeau, N. Gallego-Planas, J. Roncali, J. Am. Chem. Soc. 2003, $125,1363-1370 ; \mathrm{b})$ the procedure for the introducion of the Z-group into chalogenalcohols was used: M. Shiozaki, M. Arai, J. Org. Chem. 1989, 54, 3754-3755.

[115] The method similar to the tritylation of 2-(2-chloroethyloxy)ethanol was applied; cf.: L. Jullien, J. Canceill, L. Lacombe, J.-M. Lehn, J. Chem. Soc. Perkin Trans. 2 1994, 989-1002. [116] M. A. Pilkington-Miksa, S. Sarkar, M. J. Writer, S. E. Barker, P. A. Shamlou, S. L. Hart, H. C. Hailes, A. B. Tabor, Eur. J. Org. Chem. 2008, 2900-2914.

[117] For the conversion of the mesylate into the iodide was used slightly modified procedure from: G. Seidel, D. Laurich, A. Fürstner, J. Org. Chem. 2004, 69, 3950-3952.

[118] R. T. Blickenstaff, J. Am. Chem. Soc. 1960, 82, 3673-3676.

[119] D. Cabaret, M. Wakselman, Can. J. Chem. 1990, 68, 2253-2257.

[120] R. Mazur, G. Plume, Experientia 1968, 24, 661-661.

[121] G. Sabitha, B. V. S. Reddy, S. Abraham, J. S. Yadav, Tetrahedron Lett. 1999, 40, $1569-1570$.

[122] The procedure for the $N$-ethylation of 4-aminophthalimides was used: H. Karatani, Bull. Chem. Soc. Jpn. 1987, 60, 2023-2029.

[123] a) S. R. Adams, R. E. Campbell, L. A. Gross, B. R. Martin, G. K. Walkup, Y. Yao, J. Llopis, R. Y. Tsien, J. Am. Chem. Soc. 2002, 124, 6063-6076; b) R. Y. Tsien, A. B. Griffin, (The Regents of the University of California), WO 99/21013 A1 (29.04.1999).

[124] a) K. S. Thorn, N. Naber, M. Matuska, R. D. Vale, R. Cooke, Protein Sci. 2000, 9, $213-$ 217; b) R. D. Vale, K. S. Thorn, R. Cooke, M. Matuska, N. Naber, (The Regents of the University of California), WO 01/53325 A2 (26.07.2001).

[125] C. Hoffmann, G. Gaietta, M. Bünemann, S. R. Adams, S. Oberhoff-Maas, B. Behr, J.-P. Vilardaga, R. Y. Tsien, M. H. Ellisman, M. J. Lohse, Nat. Methods 2005, 2(3), 171-176. 
[126] O. Tour, S. R. Adams, R. A. Kerr, R. M. Meijer, T. J. Sejnowski, R. W. Tsien, R. Y. Tsien, Nat. Chem. Biol. 2007, 3, 423-431.

[127] E. Genin, O. Carion, B. Mahler, B. Dubertret, N. Arhel, P. Charneau, E. Doris, C. Mioskowski, J. Am. Chem. Soc. 2008, 130, 8596-8597.

[128] Y. Taguchi, Z.-D. Shi, D. W. Dorward, L. Greene, G. S. Baron, Mol. Biol. Cell 2009, $20,233-244$.

[129] P. Lohse, M. C. Wright, M. Mcpherson (Phylos, Inc.), WO 00/72869 A1 (07.12.2000).

[130] J. Nakanishi, M. Maeda, Y. Umezawa, Anal. Sci. 2004, 20, 273-278.

[131] C. C. Spagnuolo, R. J. Vermeij, E. A. Jares-Erijman, J. Am. Chem. Soc. 2006, 128, $12040-12041$.

[132] K. Stroffekova, C. J. Proenza, K. G. Beam, Pflüg. Arch. Eur. J. Phy. 2001, 442, 859866.

[133] H. Cao, B. Chen, T. C. Squier, M. U. Mayer, Chem. Com. 2006, 2601-2603.

[134] J. Nakanishi, T. Nakajima, M. Sato, T. Ozawa, K. Tohda, Y. Umezawa, Anal. Chem. 2001, 73, 2920-2928.

[135] G. Gaietta, T. J. Deerinck, S. R. Adams, J. Bouwer, O. Tour, D. W. Laird, G. E. Sosinski, R. Y. Tsien, M. H. Ellisman, Science 2002, 296, 503-507.

[136] S. Miller (University of Massachusetts), WO 08/109463 A2 (12.09.2008).

[137] S. Miller (University of Massachusetts), WO 2009/036351 A2 (19.03.2009).

[138] M. Adamczyk, J. Grote, and J. A. Moore, Bioconjugate Chem. 1999, 10, 544-547.

[139] V. N. Belov, M. L. Bossi, J. Fölling, V. P. Boyarskiy, and S. W. Hell, Chem. Eur. J. 2009, 15, $10762-10776$

[140] S. M. Polyakova, unpublished results.

[141] F.-S. Wu, Planta 1987, 171, 346-357.

[142] S. Jakobs, M. Andresen, C. A. Wurm. "'FLASH' protein labeling" In Probes and Tages to Study Biomolecular Function, Wiley-VCH (Weinheim, Germany), 2008, pp. 73-88. 


\section{Spectral Data}

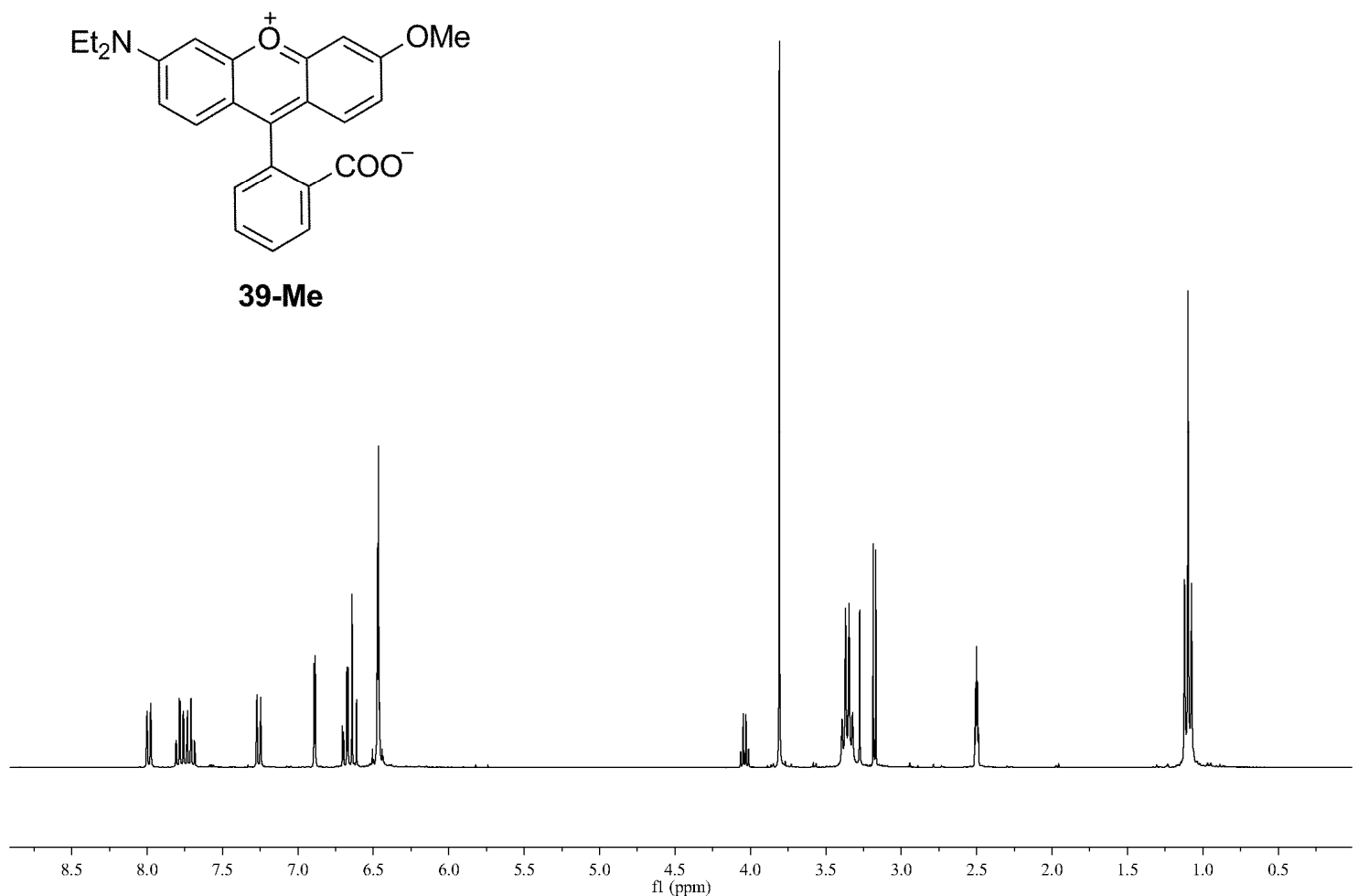

${ }^{1} \mathrm{H}$ NMR (300 MHz, [D $]$-DMSO)
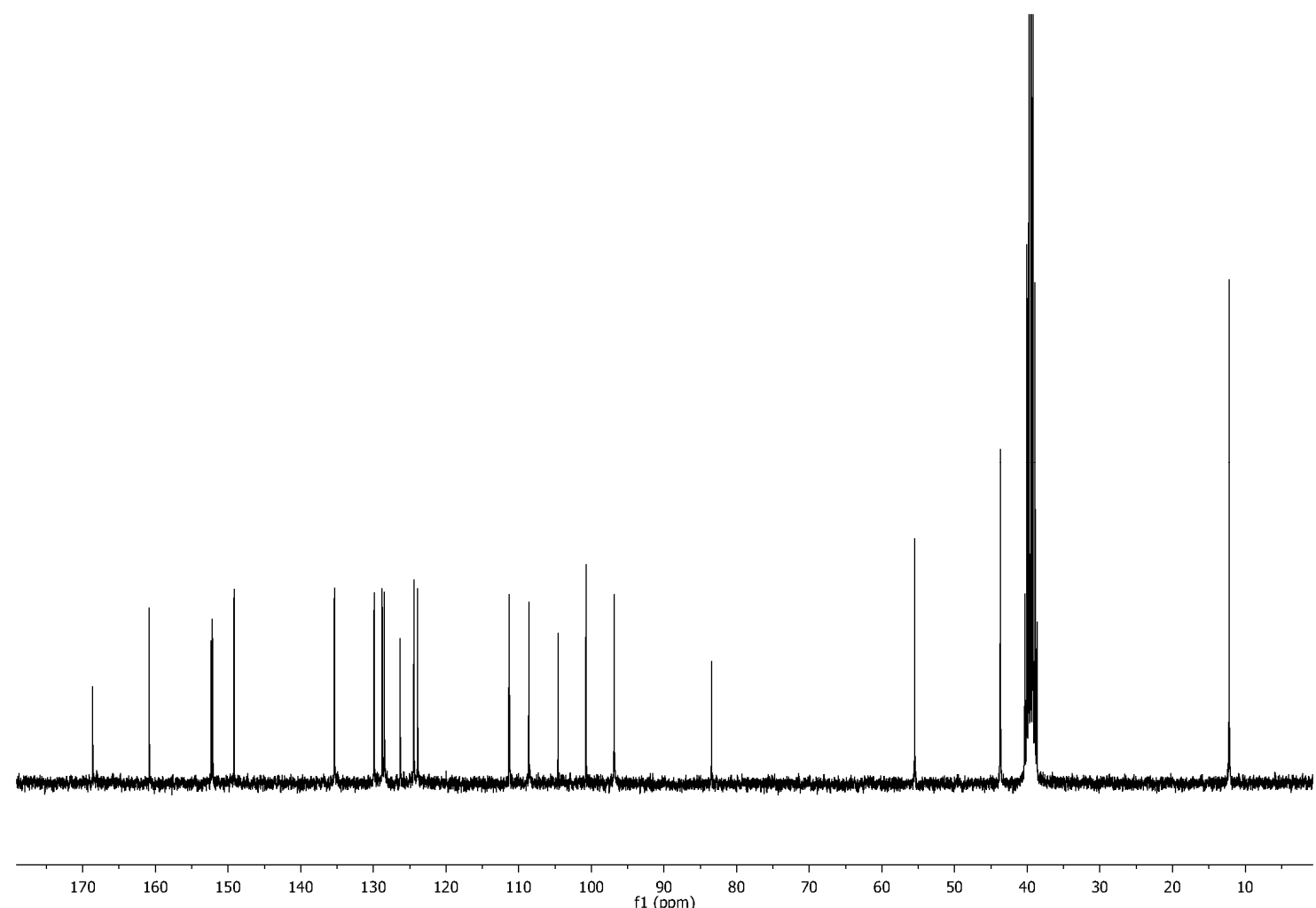

${ }^{13} \mathrm{C}$ NMR (75 MHz, [D $]$-DMSO) 


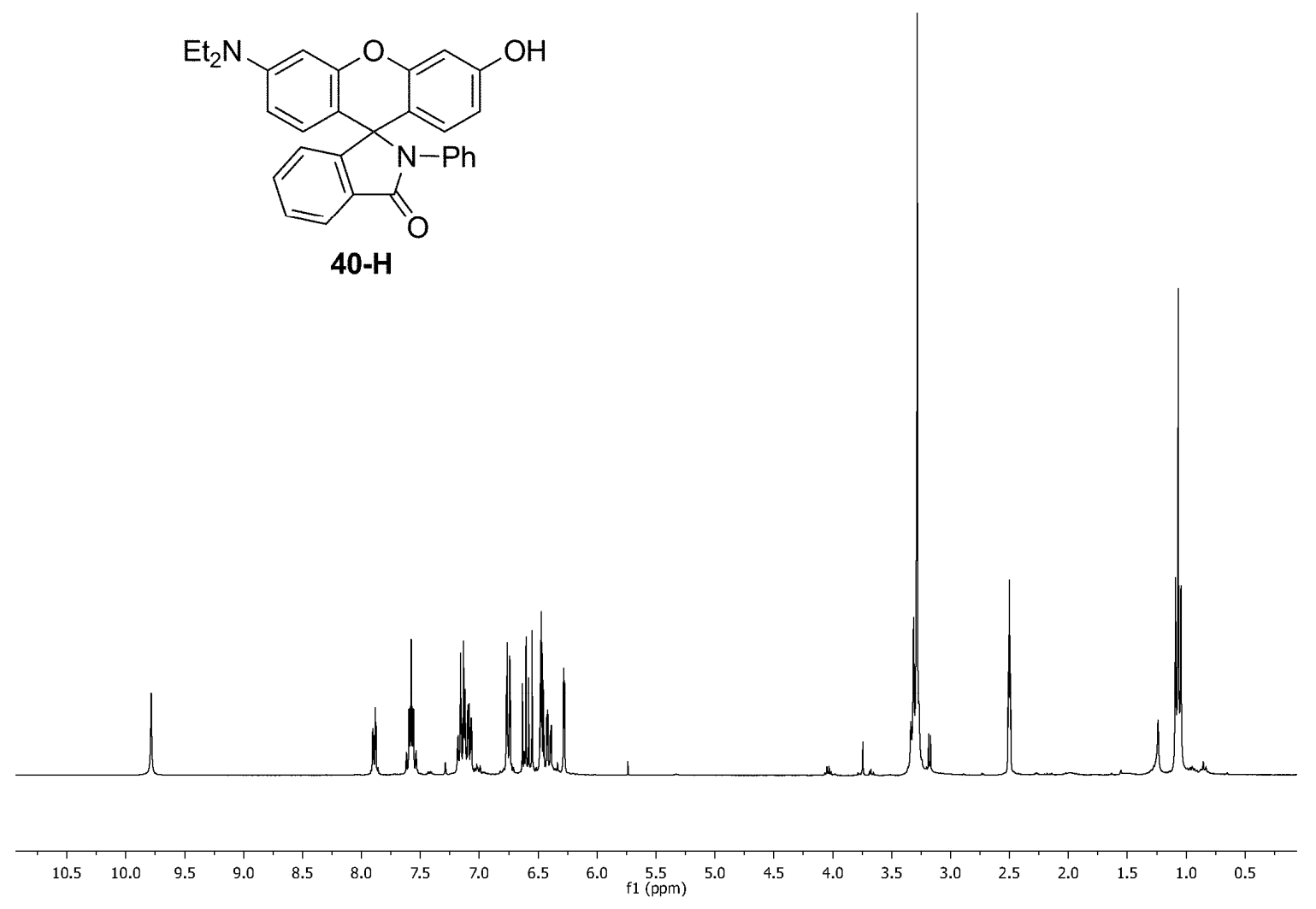

${ }^{1} \mathrm{H}$ NMR (300 MHz, [D 6 -DMSO)

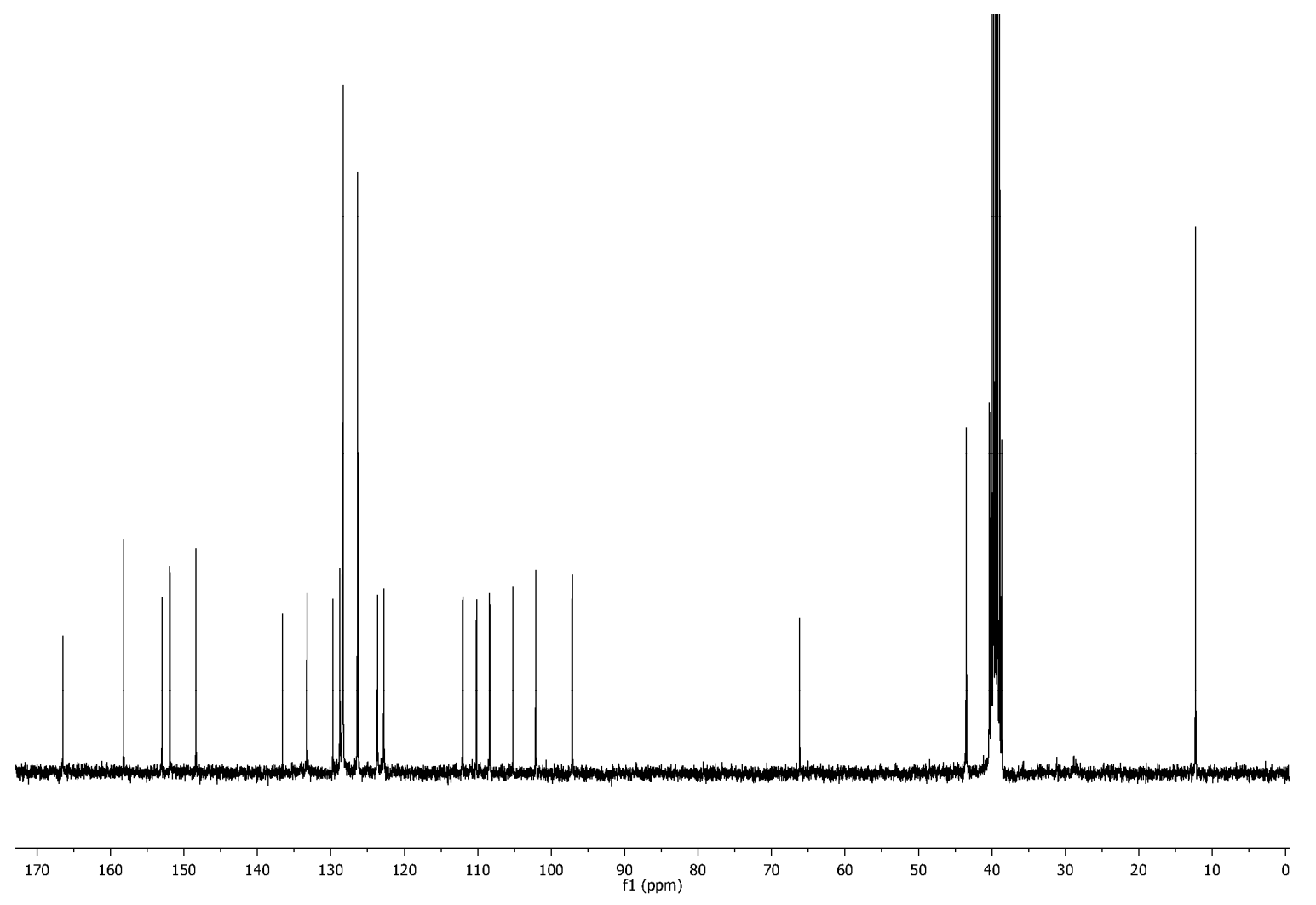

${ }^{13} \mathrm{C}$ NMR (75 MHz, [D 6 -DMSO) 

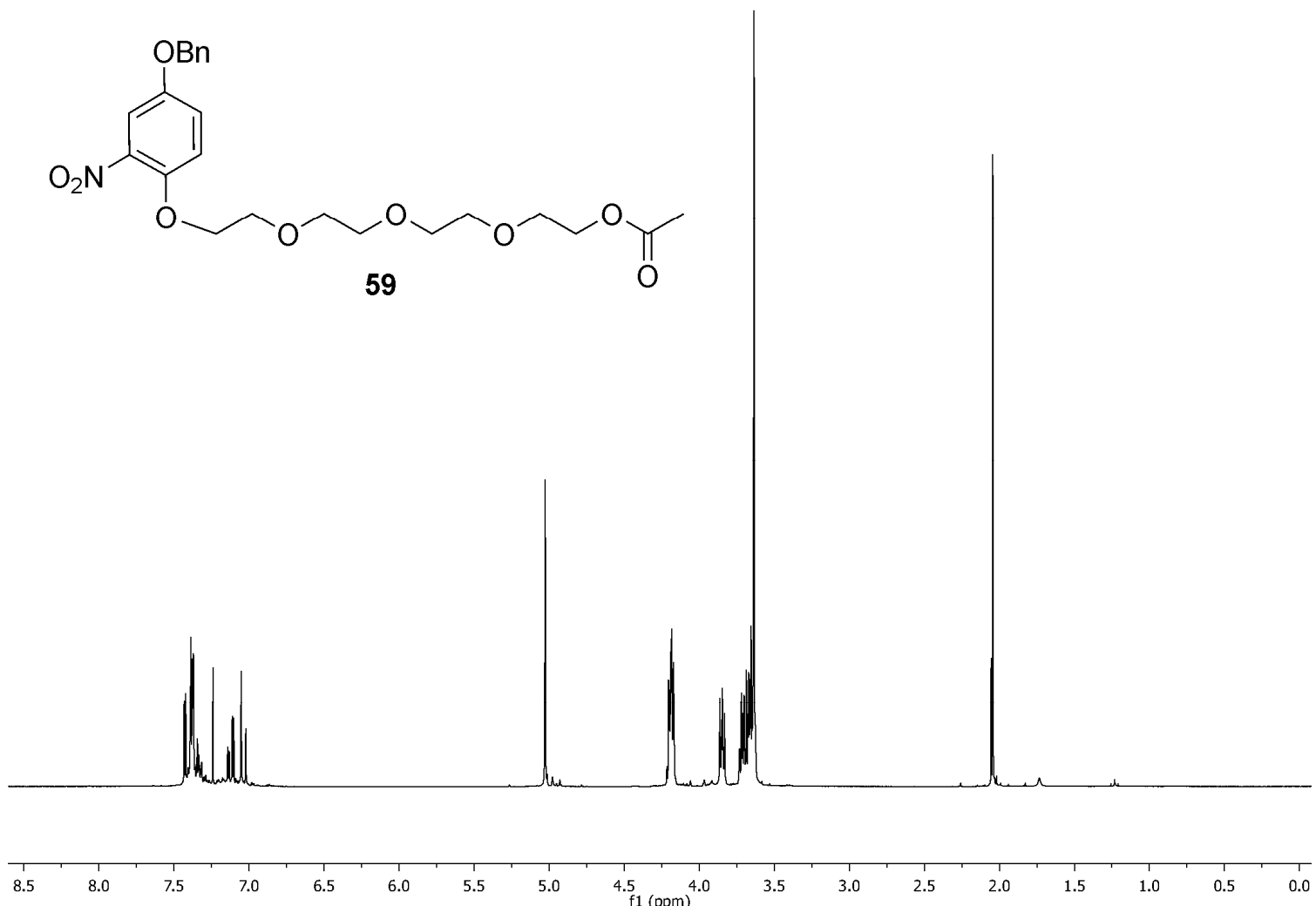

${ }^{1} \mathrm{H} \mathrm{NMR}\left(300 \mathrm{MHz}, \mathrm{CDCl}_{3}\right)$

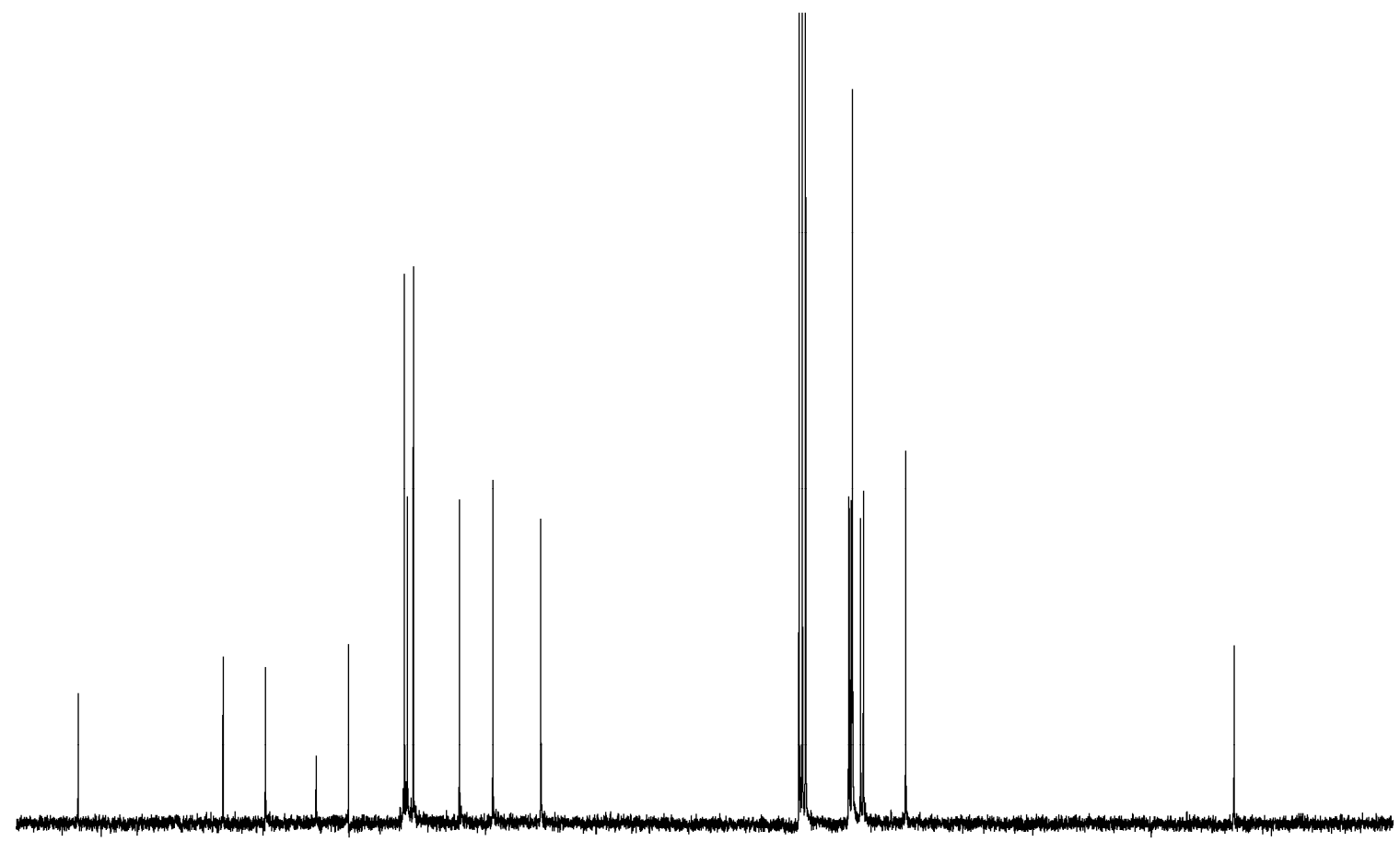

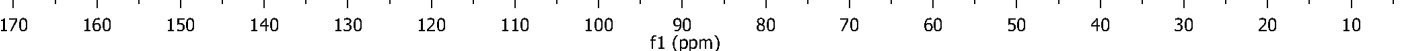

${ }^{13} \mathrm{C}$ NMR $\left(75 \mathrm{MHz}, \mathrm{CDCl}_{3}\right)$ 


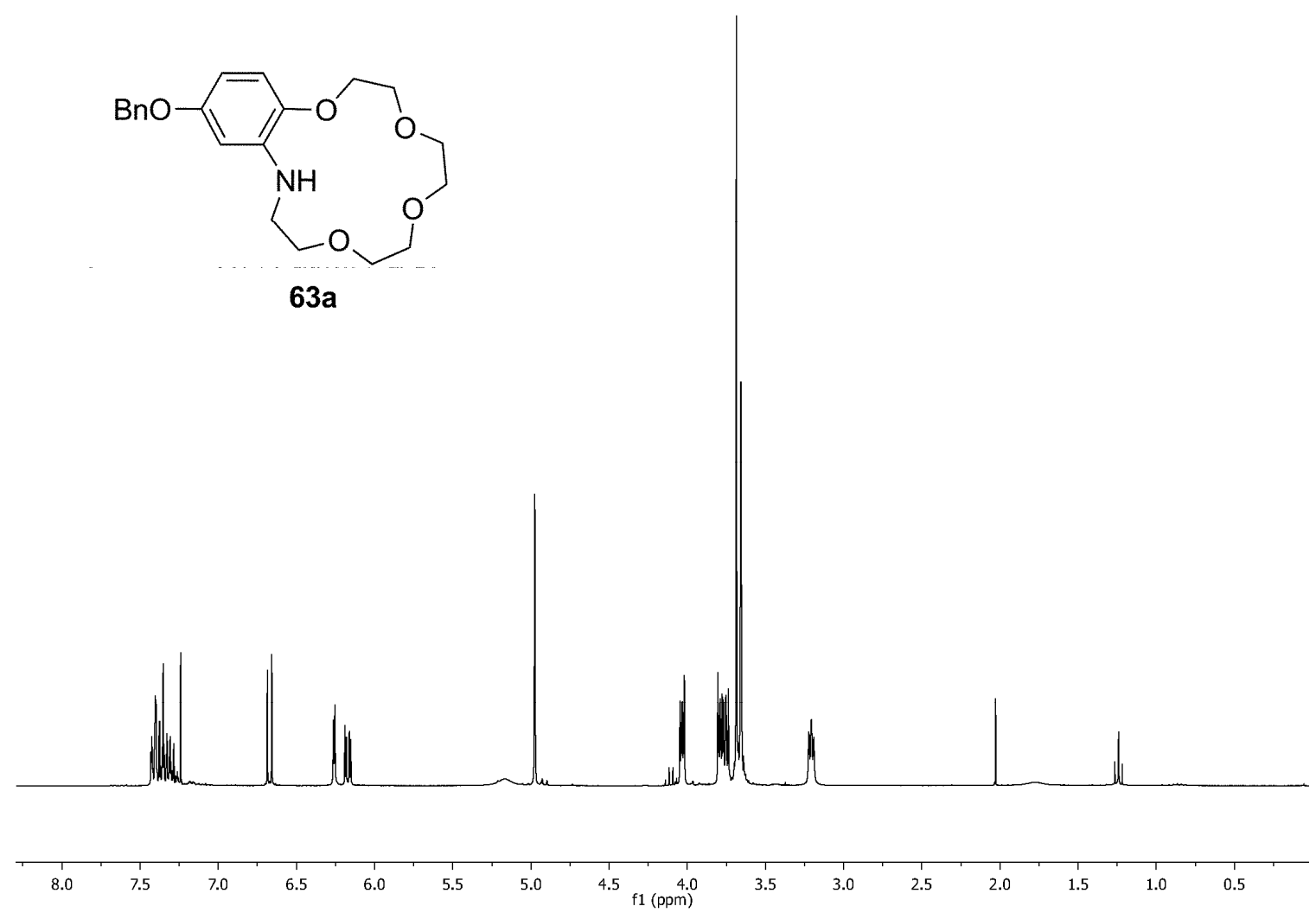

${ }^{1} \mathrm{H}$ NMR (300 MHz, $\mathrm{CDCl}_{3}$ )

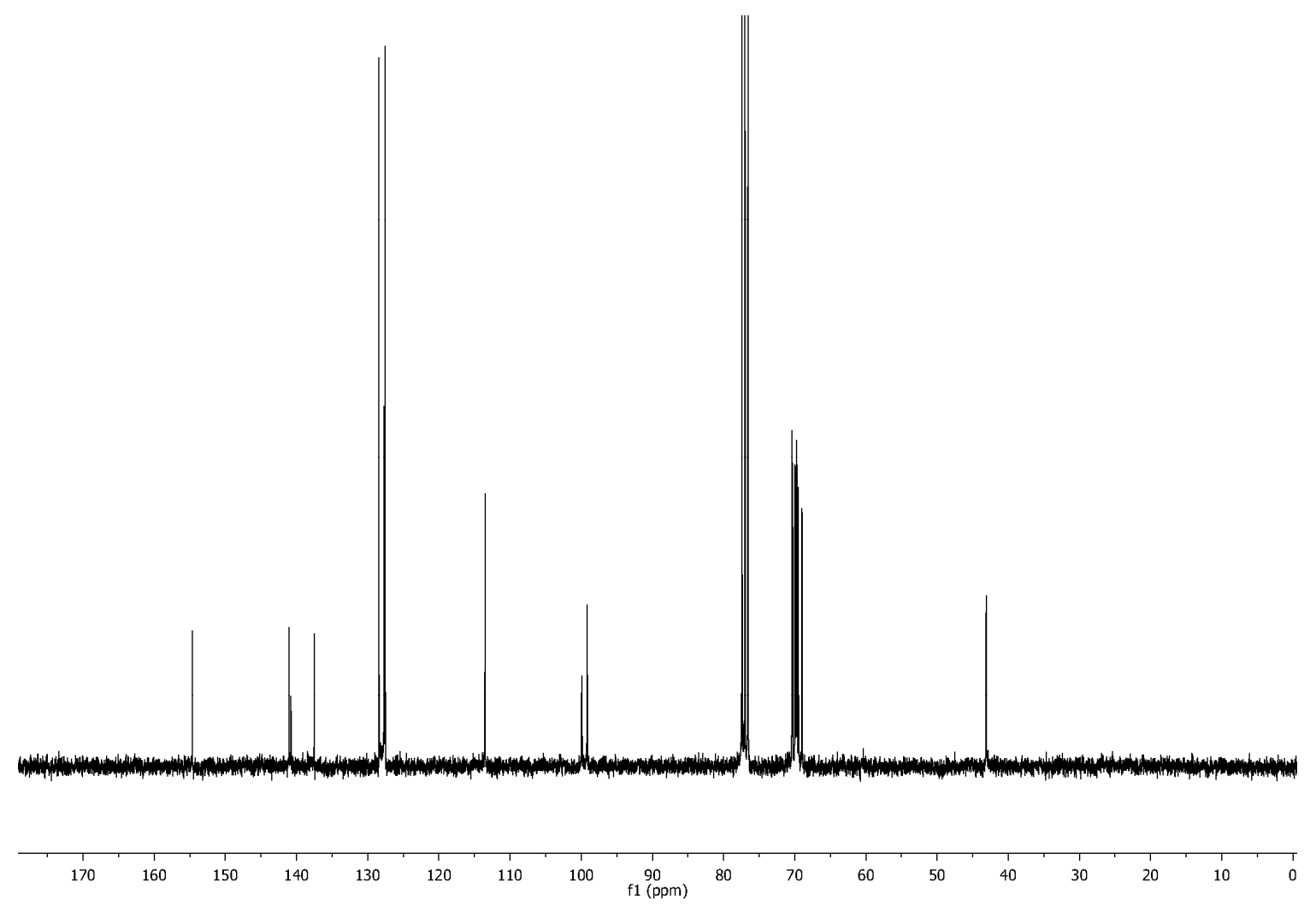

${ }^{13} \mathrm{C}$ NMR $\left(75 \mathrm{MHz}, \mathrm{CDCl}_{3}\right)$ 


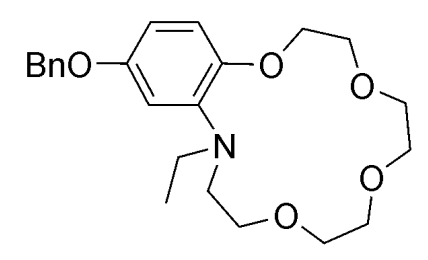

$63 c$
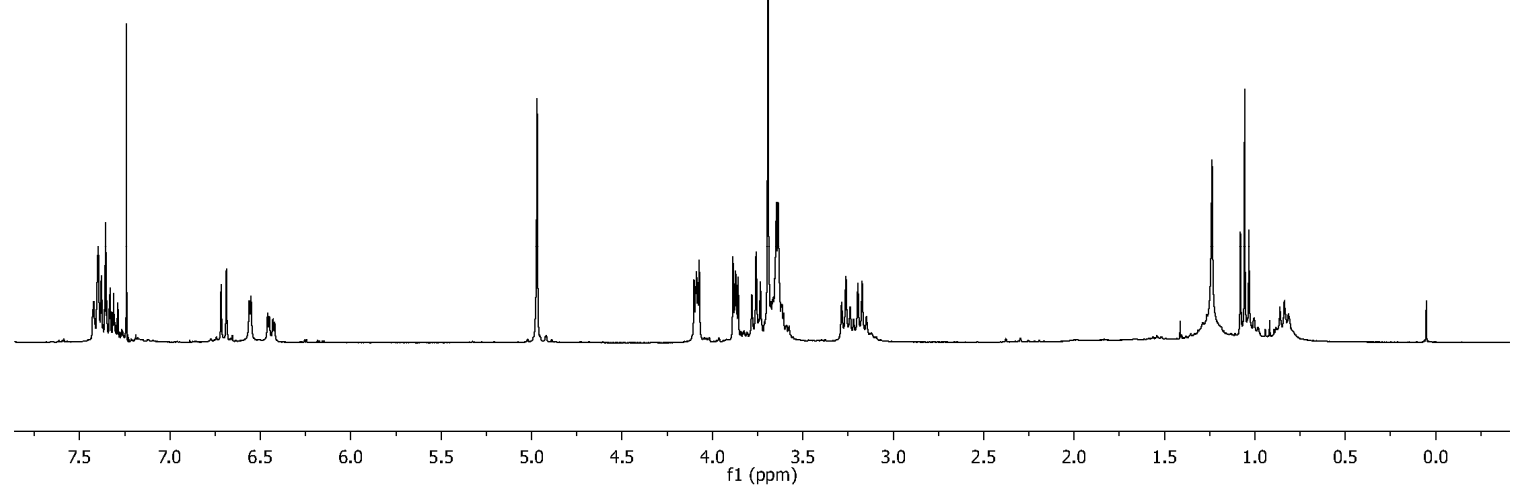

${ }^{1} \mathrm{H}$ NMR $\left(300 \mathrm{MHz}, \mathrm{CDCl}_{3}\right)$

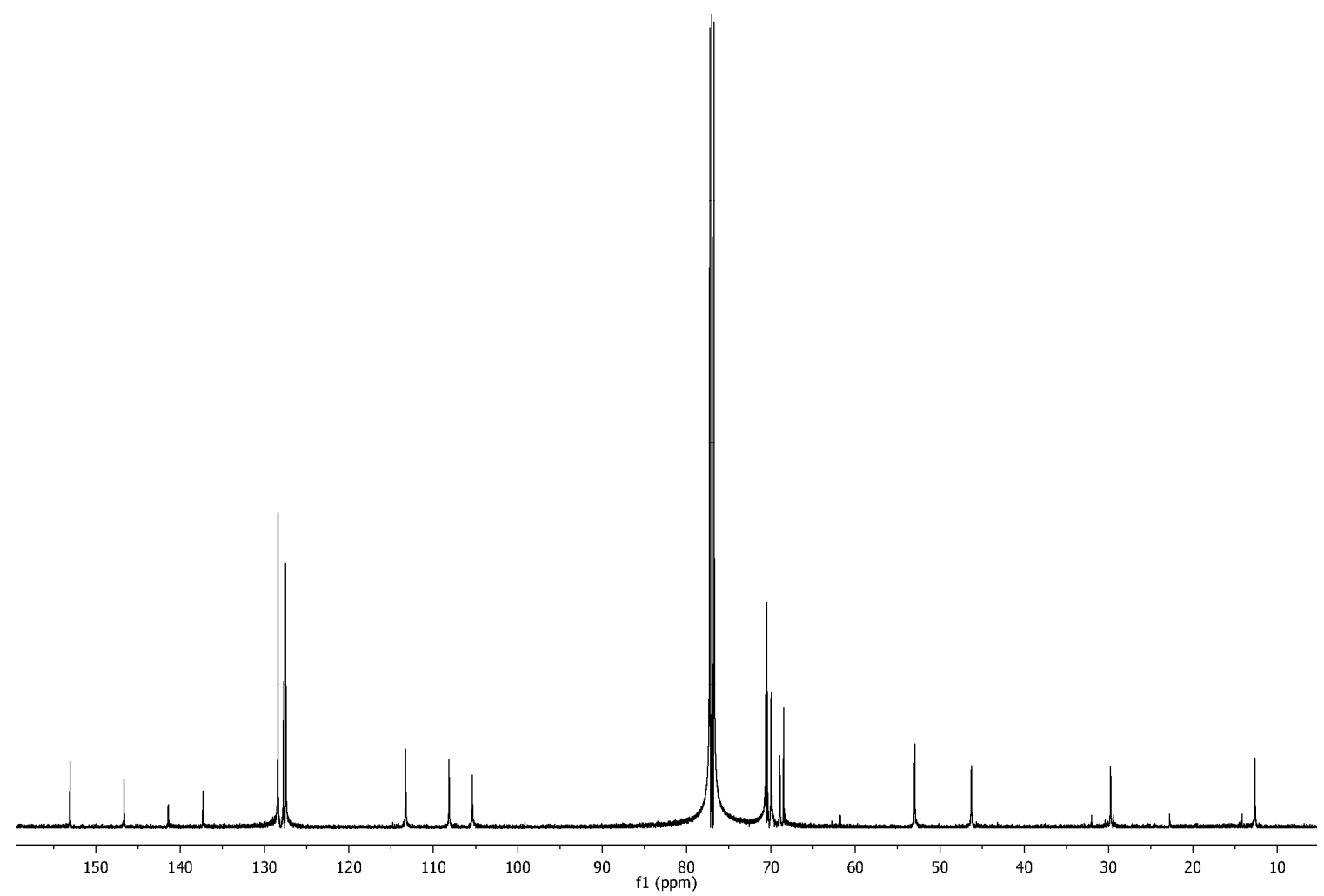

${ }^{13} \mathrm{C}$ NMR $\left(126 \mathrm{MHz}, \mathrm{CDCl}_{3}\right)$ 

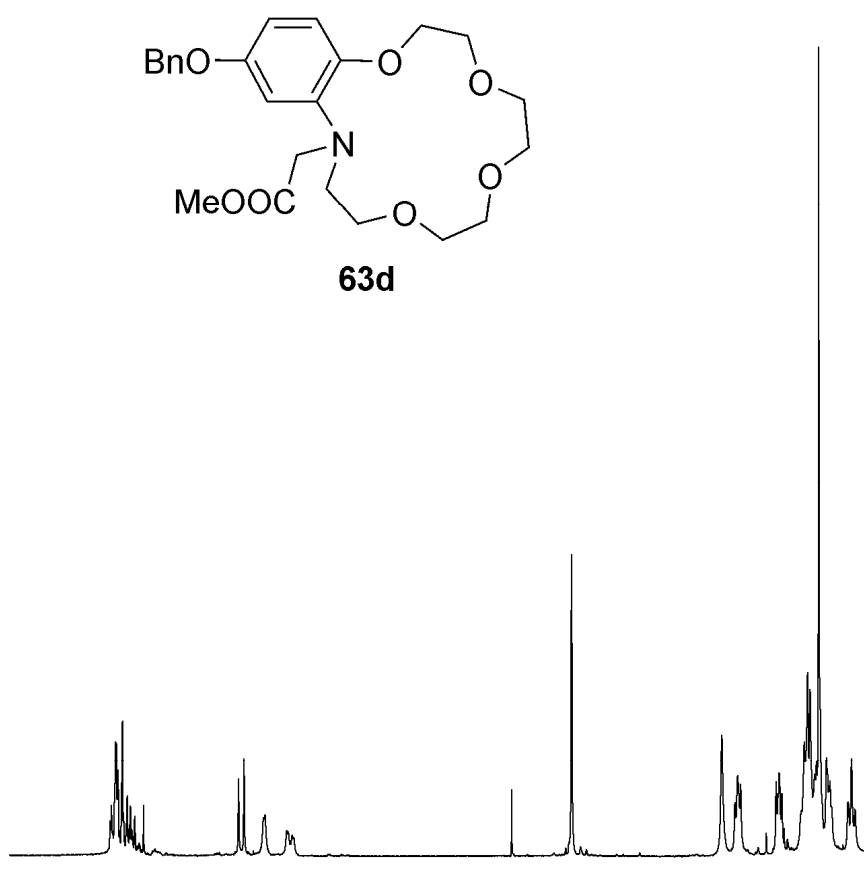

${ }^{1} \mathrm{H}$ NMR (300 MHz, $\mathrm{CDCl}_{3}$ )

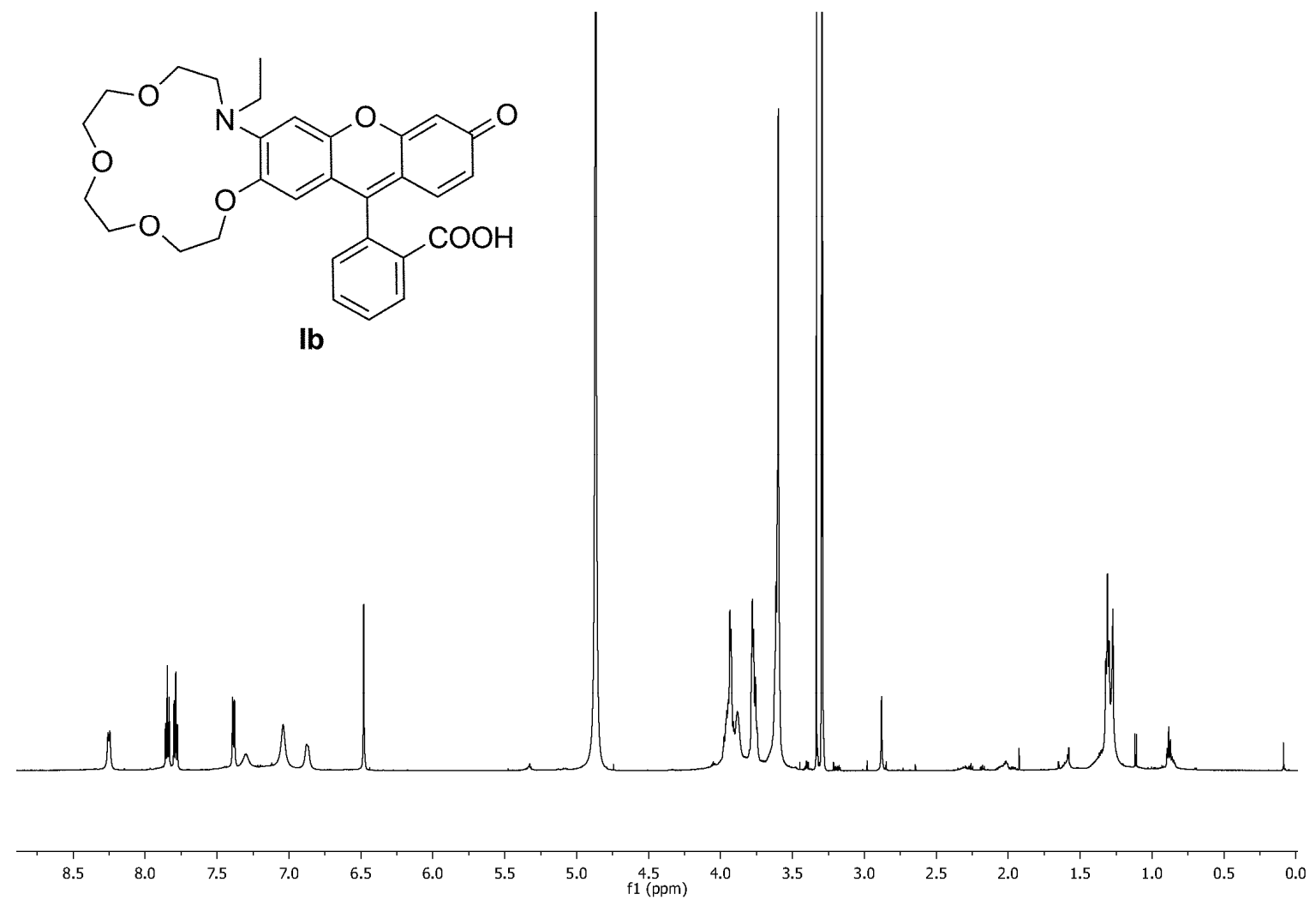

${ }^{1} \mathrm{H}$ NMR (600 MHz, CD 3 OD) 


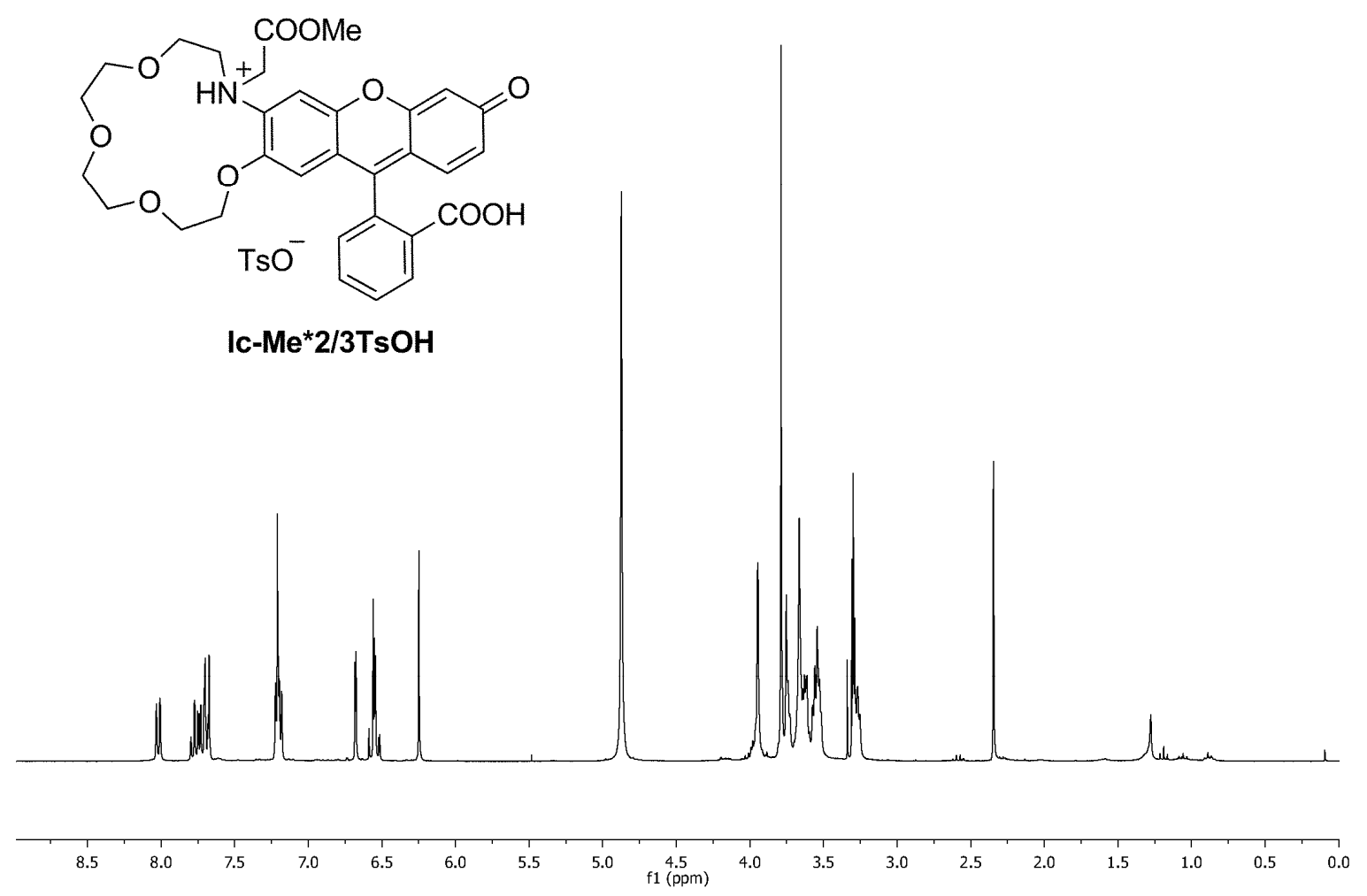

${ }^{1} \mathrm{H}$ NMR (300 MHz, $\left.\mathrm{CD}_{3} \mathrm{OD}\right)$

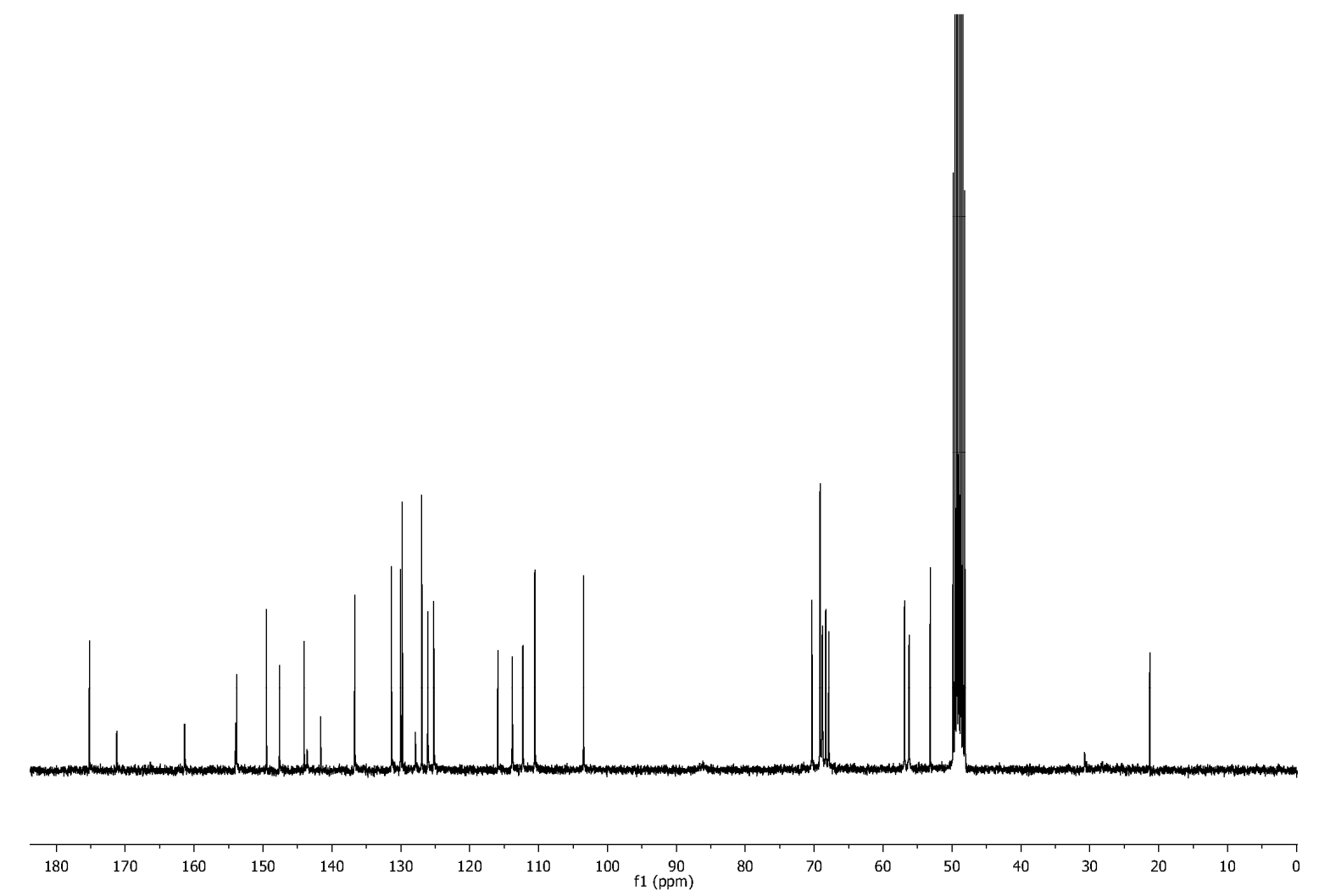

${ }^{13} \mathrm{C}$ NMR $\left(126 \mathrm{MHz}, \mathrm{CD}_{3} \mathrm{OD}\right)$ 


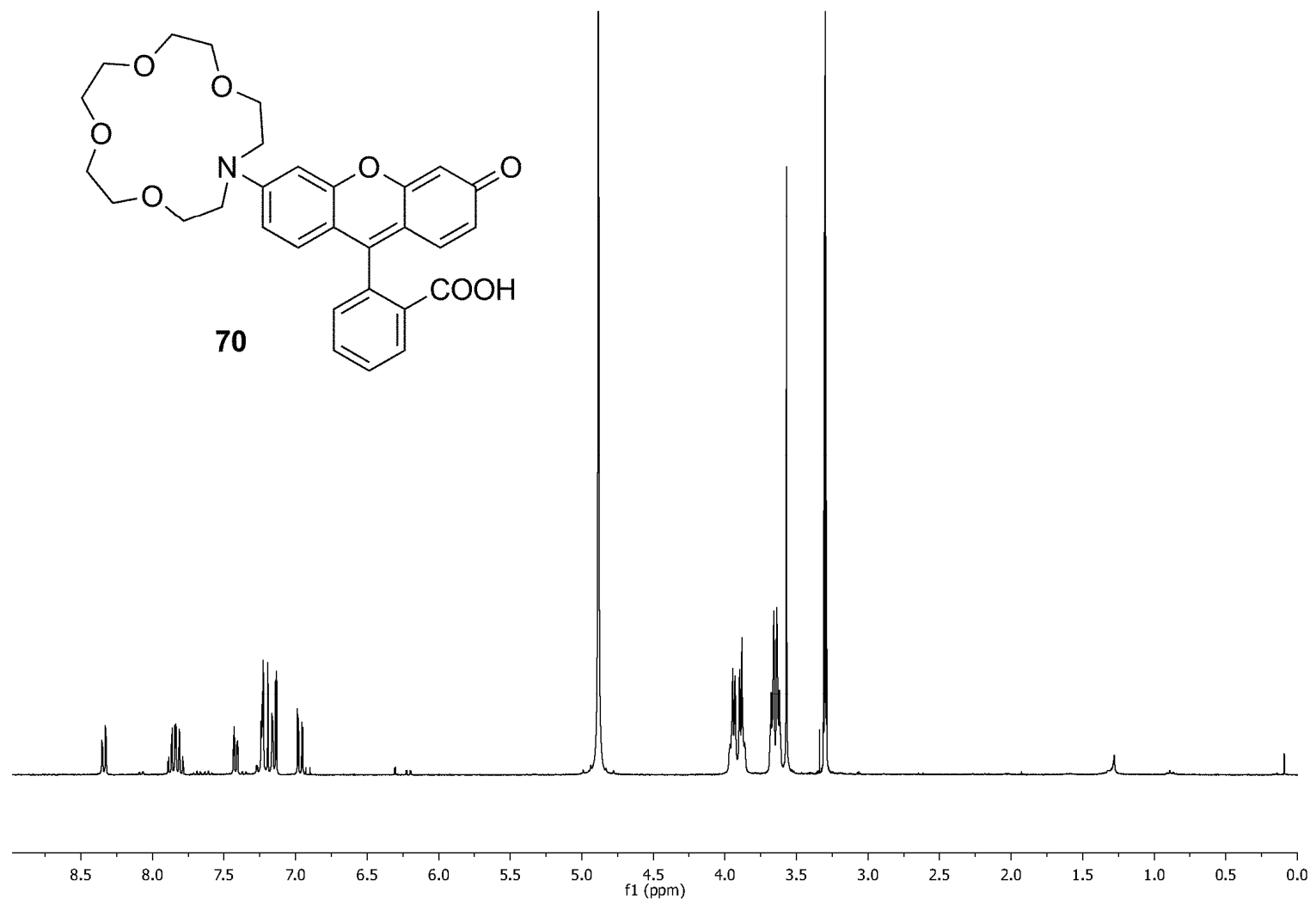

${ }^{1} \mathrm{H}$ NMR (300 MHz, CD 3 OD)

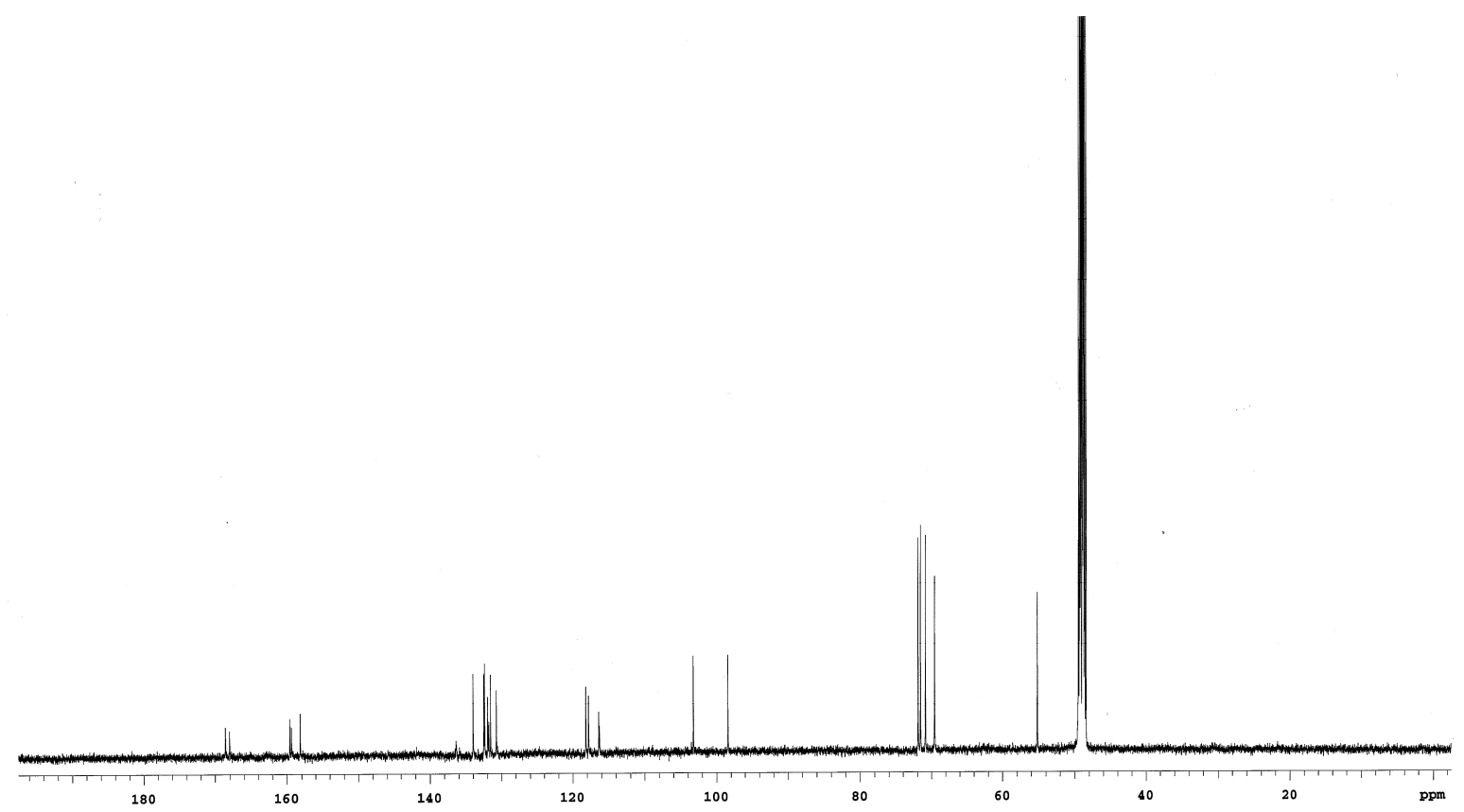

${ }^{13} \mathrm{C}$ NMR (126 MHz, $\left.\mathrm{CD}_{3} \mathrm{OD}\right)$ 


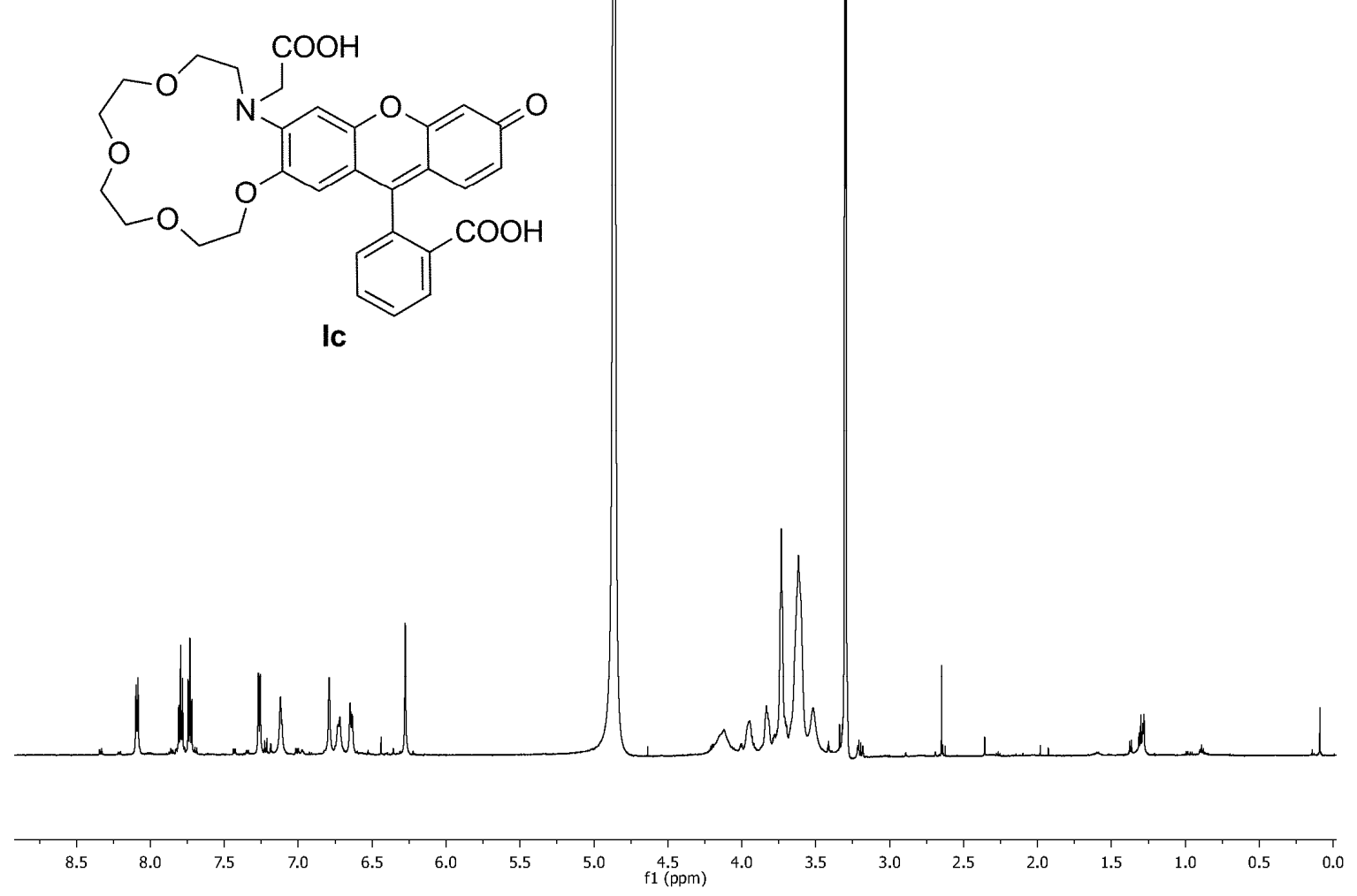

${ }^{1} \mathrm{H}$ NMR (600 MHz, CD 3 OD)<smiles>CN1C(=O)c2cc(OCCOCCOCCOCCO[18F])c([N+](=O)[O-])cc2C1=O</smiles>
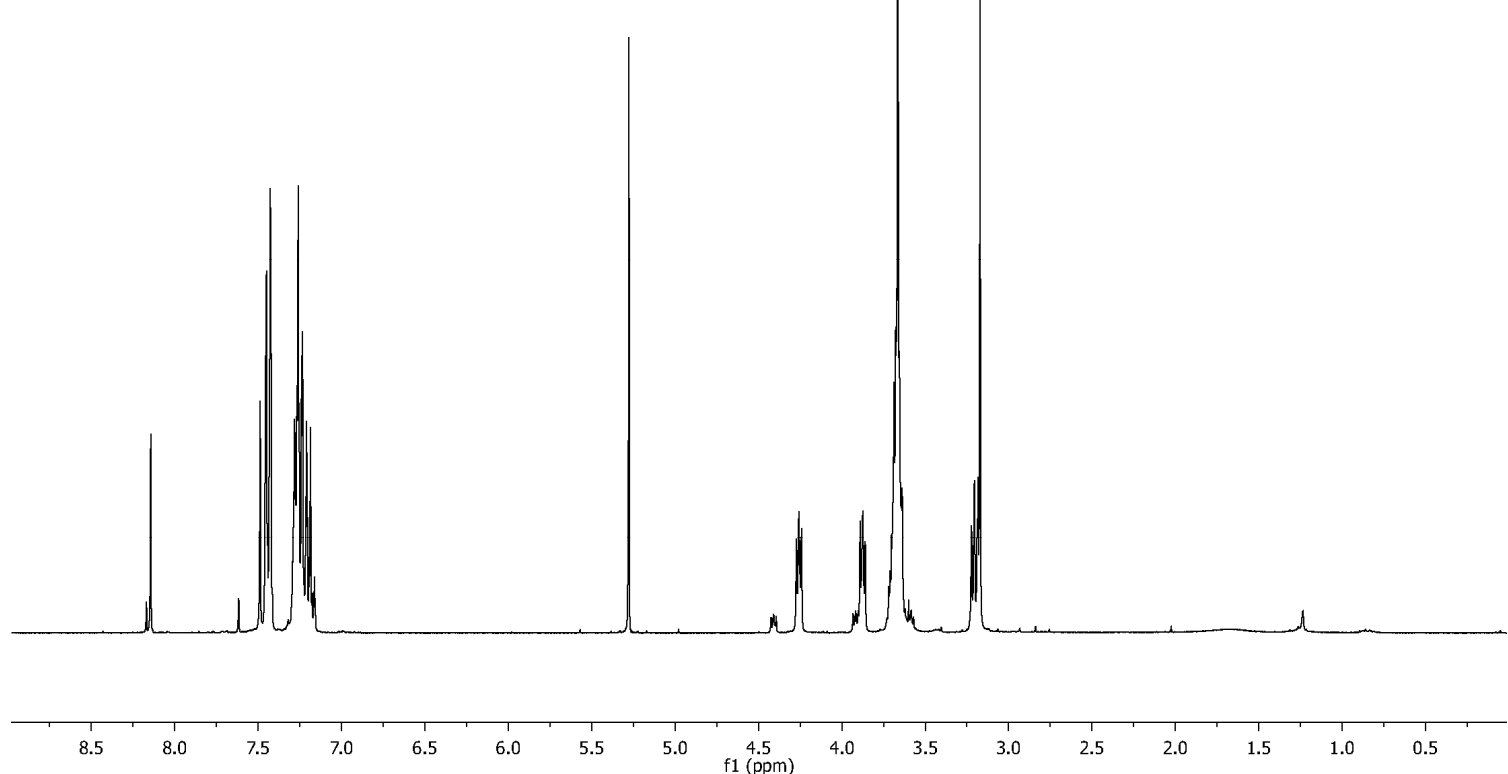

${ }^{1} \mathrm{H}$ NMR $\left(300 \mathrm{MHz}, \mathrm{CDCl}_{3}\right)$ 


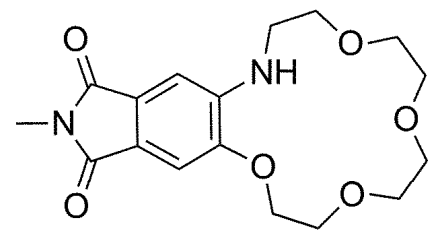

92
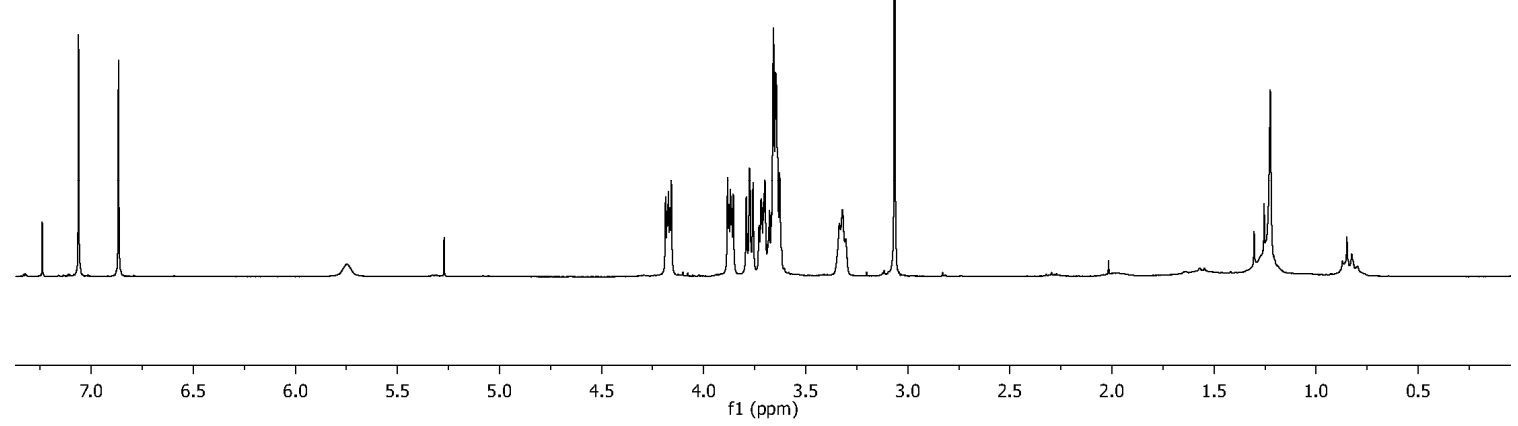

${ }^{1} \mathrm{H}$ NMR $\left(300 \mathrm{MHz}, \mathrm{CDCl}_{3}\right)$

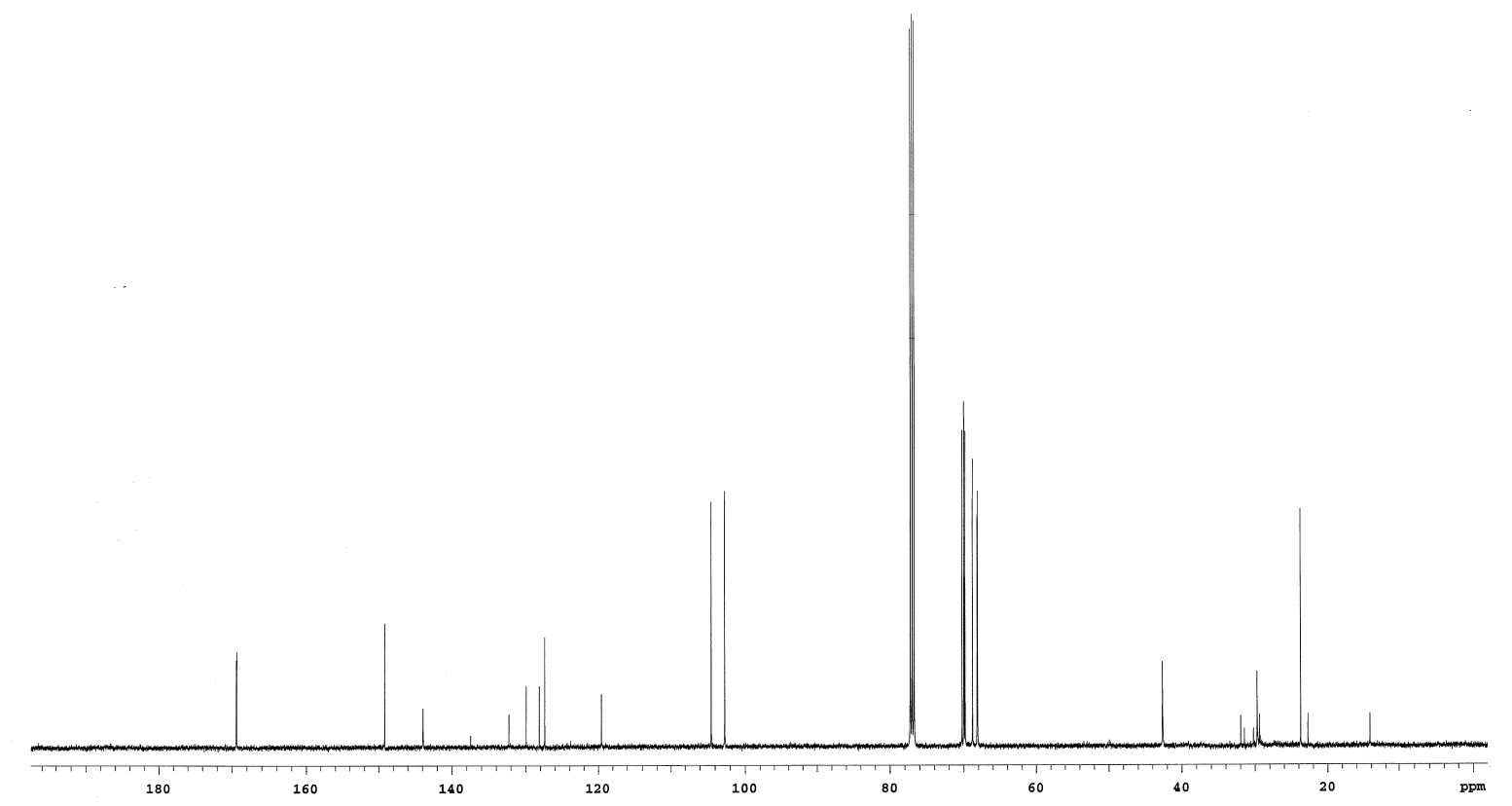

${ }^{13} \mathrm{C}$ NMR $\left(126 \mathrm{MHz}, \mathrm{CDCl}_{3}\right)$ 
<smiles>CCN1CCOCCOCCOCCOc2cc3c(cc21)C(=O)N(C)C3=O</smiles>

92-Et
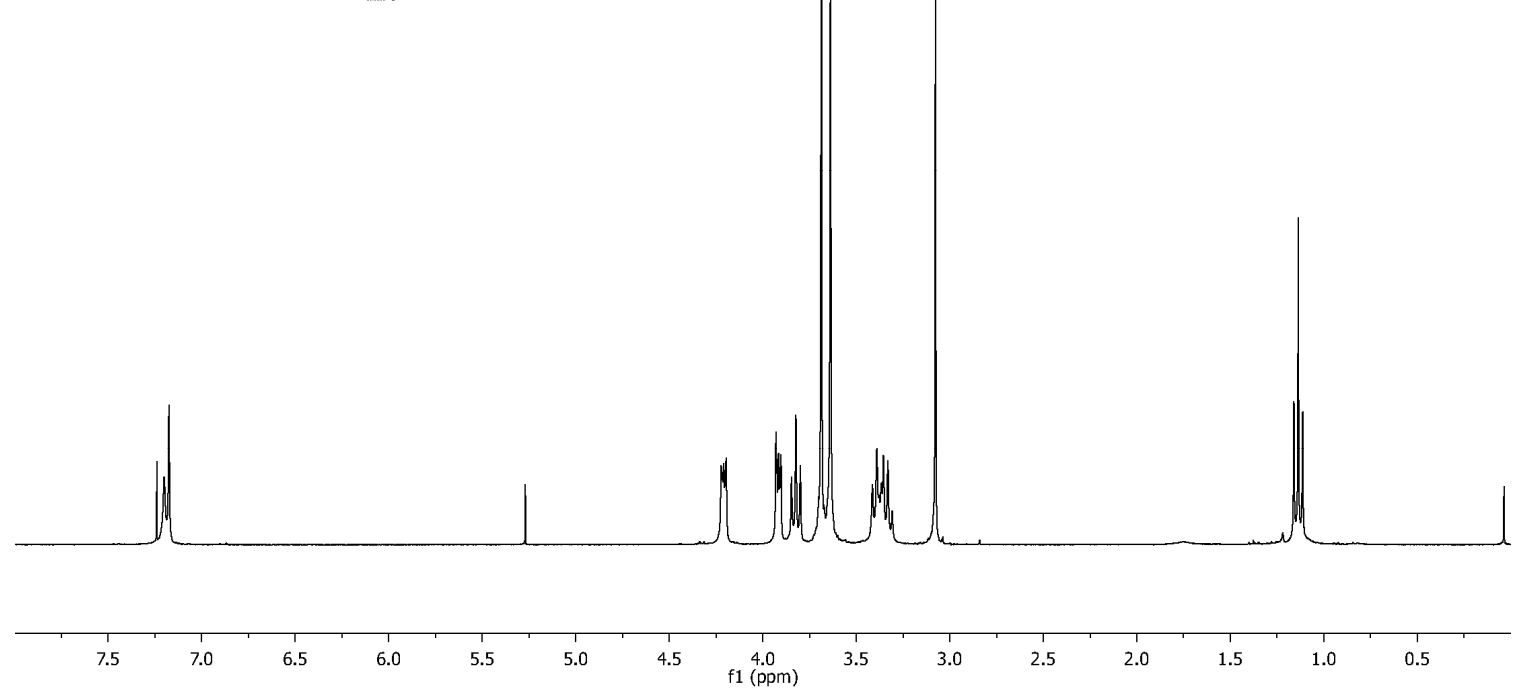

${ }^{1} \mathrm{H}$ NMR (300 MHz, $\mathrm{CDCl}_{3}$ )
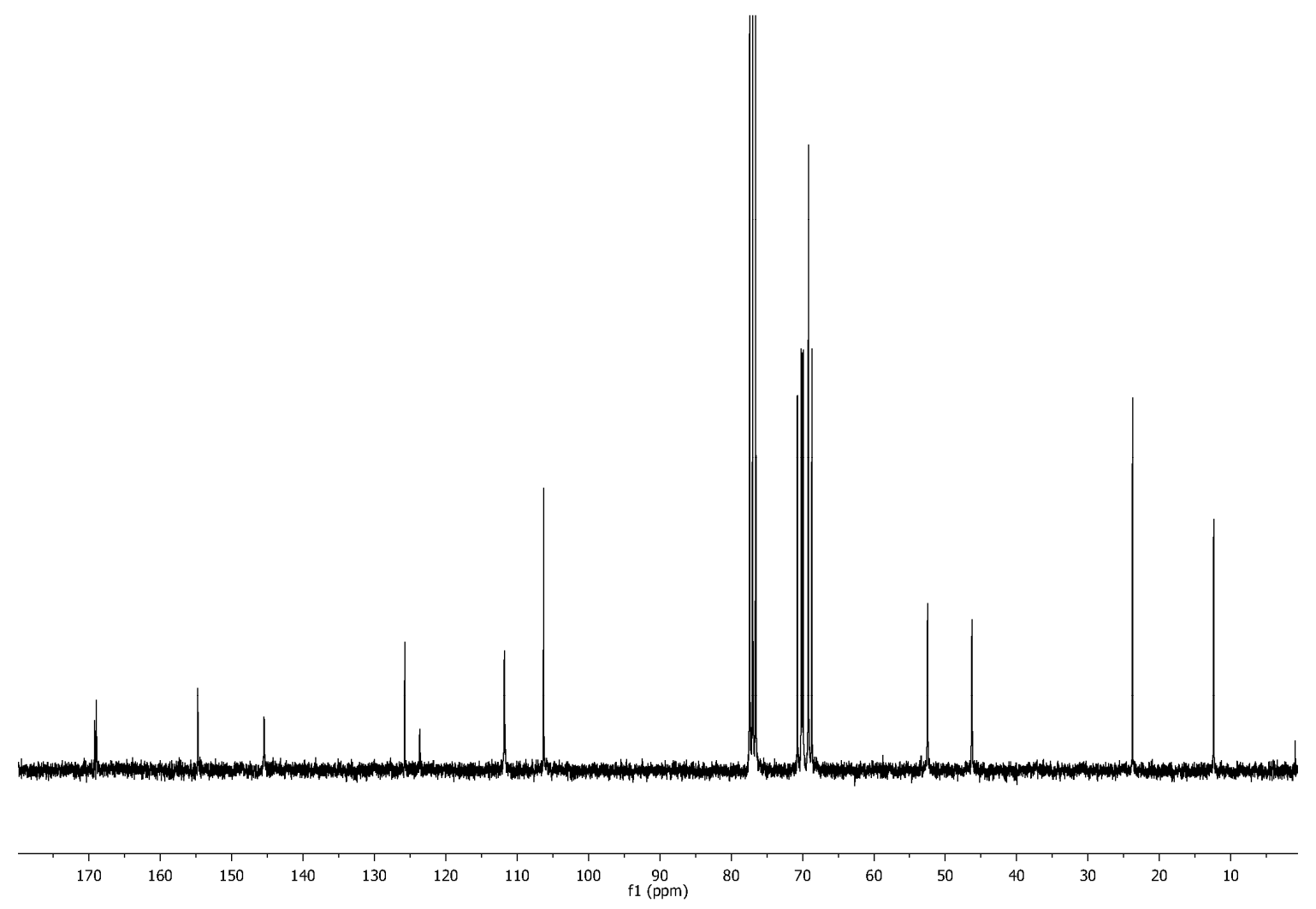

${ }^{13} \mathrm{C}$ NMR $\left(75 \mathrm{MHz}, \mathrm{CDCl}_{3}\right)$ 


\section{Acknowledgements}

I would like to thank:

- Prof. Dr. Stefan W. Hell for giving me an opportunity to work in his excellent group, for his help and financial support during the difficult period of work on my $\mathrm{PhD}$ thesis, for the perfect working conditions in his department.

- my supervisor Prof. Dr. A. de Meijere for leadership, patience, appreciation and help in my thesis preparation.

- my laboratory advisor Dr. Vladimir Belov for his patience and readiness to work with me, for the leadership and professional approach to the organic synthesis, for the interesting discussions and advice, for the development of my character as a scientist, for the all-round support and help in my work.

- Prof. Dr. Mariano Bossi for the spectroscopic experiments, interest and productive discussions on scientific problems in the cooperative work, for the help in the preparation of numerous plots for my thesis.

- Priv.-Doz. Stefan Jacobs and Dr. Stefan Stoldt for the cooperation, interesting discussions and productive work in the area of selective labeling of proteins in living cells.

- the NMR-team and its head Dipl.-Chem. R. Machinek for the fast and precise measurements and discussion of NMR problems, Dr. H. Frauendorf and Mrs. G. Udvarnoki for MS and HR-MS measurements and helpful discussions of complicated cases, Mr. F. Hambloch for the elemental analysis, D. Ouw for the measurements of the fluorescence and absorption spectra, Nina Ohm for the measurements of some photophysical properties of the prepared compounds, Mr. Gerhard Busse for giving me the opportunity to work with very precise fluorimeter and help with the study of this equipment, Prof. Dr. D. Möbius for the assistance in work with the program Origin ${ }^{\circledR}$.

My thanks also go to Dr. Katrin Willig and Dr. Birka Lalkens for their help in the understanding of the basics of STED-nanoscopy.

I am very thankful to my colleagues Dr. Svetlana Polyakova and Dr. Gyuzel Mitronova for the invaluable "life experience" and "life wisdom", their unquenchable optimism, which put me in a good mood in hard situations.

I thank Dr. V. Boyarskiy for his advice, support, scientific and historical discussions.

I am very grateful to all group members of the department of NanoBiophotonics, especially Dr. Vladimir Belov, Dr. Svetlana Polyakova, Dipl.-Chem. Roman Kunetsky, Prof. Dr. Mariano Bossi, Dr. Gyuzel Mitronova, Dipl.-Chem. Sevil Sejtablaeva, Dr. Kirill Kolmakov, Dr. Heiko Schill, Dr. Shamil Nizamov, Dr. Jan Marian von Hof and Dipl.-Chem. Maxim Sednev for their friendly laboratory atmosphere.

I would like to thank Dr. Kirill Kolmakov for the interesting scientific and historical discussions, for his moral support and for "tea-help" from Russia. 
I appreciate the assistance and help from Mrs. Anja Dietrich, Ms. Josephine Stadler, Mrs. Sarah Aschermann, Ms. Alena Stefan and Mrs. Berta Hampel in the solving of many organizational problems in the course of my work and study.

My thanks go to the Otto-Hahn-Bibliothek-team at the Max-Planck-Institute and its head Bernard Reuse for the unlimited help with the search of scientific literature.

I am also very grateful to Mikhail Zhukovkiy, Denis Yalalov, Vadim Korotkov, Katya Zarudnaya, Sergey Korneev, Alexander Lygin, Antonina Lygina, Tanya Sergeeva, Mikhail Shaposhnikov, Mariana Balan, Chaytaniya Ullal, Valentin Rassadin, Alena Pan'kova, Alexey Galenko, Dmitrij Dar'in, Alexander Korolev for the friendship and moral support during the work in Göttingen, for the funny parties and nice trips to European countries during the free time.

I am very thankful to Dr. Pavel S. Lobanov for his moral support and faith in my abilities.

I thank Dr. Christian Wurm for his readiness to help in many biological questions and interesting scientific discussions.

I am very grateful to Dr. Heiko Schill, Dr. Kirill Kolmakov and especially Dr. Vladimir Belov for the careful proof-reading of this manuscript.

Dr. Heiko Schill and Dr. Marco Roose were of great help with all computer problems.

I thank Prof. Dr. Stefan W. Hell for financial support in my German courses at the Volkshochschule (VHS).

I am very grateful to my teachers at the VHS: Frau Krause, Tanya Vert, and University Dozent Alexandra Hensel for their great help and support in the study of the German language from the "zero"-level.

I appreciate the help from Dr. Heiko Schill in the correction of my German letters and documents, and finally,

my thanks also go to Hannes Bock for multiple discussions and the watching-together of many interesting soccer games. 


\section{$\underline{\text { Lebenslauf }}$}

Name

Geburtsdatum

Geburtsort

Staatsangehörigkeit

Familienstand

Anschrift

Schulbildung

Hochschulausbildung

Stipendien und Auszeichnungen
Sergey Yan

07.05 .1984

Leningrad, UdSSR

russisch

ledig

Hannoversche Straße, 103, App. 64, 37077, Göttingen Tel.: +49(170)6620974

E-mail:syan1@gwdg.de

09.1991 - 09.1999 Gesamtschule N 134, Sankt-Petersburg, Rußland

$09.1999-06.2001 \quad$ Gesamtschule N 187, Sankt-Petersburg, Rußland

$09.2001-06.2006 \quad$ Sankt-Petersburger Staatliche Universität, Rußland Fachbereich: Chemie;

Schwerpunkt: Organische Chemie;

Thema der Diplomarbeit: "Cyclokondensation von Ethyl-3,3-diaminoacrylat mit einen Pyrimidinring erhaltenden ortho-Chlorketonen und orthoChlornitrilen"

Studienabschluss als Dipl.-Chemiker mit ausgezeichneter Bewertung (Rotes Diplom), Gesamtnote: Sehr gut

Ab September 2006

Doktorand (Abteilung NanoBiophotonics, MaxPlanck Institut für Biophysikalische Chemie, Am Fassberg, 11, Göttingen, 37077, Germany)

Mai 2001

Silbermedaille (Diplom der II Klasse) bei der Internationalen chemischen Mendeleev Olympiade für Schüler (Moskau);

$04.2006-09.2006$

Stipendium der ChemBridge Corporation;

$04.2006-09.2006$

Zweites Stipendium der ChemBridge Corporation, so wie eine Belohnung bei der "Allrussischen chemischen Olympiade für Studenten und junge Wissenschaftler" und eine private Belohnung für die Teilnahme an der Tagung „Advanced Science in Organic Chemistry“

09.2006-09.2009 Doktoranden-Stipendium von der Max-PlanckGesellschaft 


\section{Publikationen:}

1. S. F. Yan; A. Yu. Ivanov, D. V. Dar'in, P. S. Lobanov, A. A. Potekhin, Direction of hydrolysis of esters of some pyrimidine-5-carboxylic acids. Chemistry of Heterocyclic Compounds (New York, NY, United States), 2007, 43(11), 1479-1480.

2. S. F. Yan, D. V. Dar'in, P. S. Lobanov, A. A. Potekhin, Cyclocondensation of ethyl 3,3diaminoacrylate with aromatic ketones and nitriles containing a labile halogen atom in the ortho position. Chemistry of Heterocyclic Compounds (New York, NY, United States), 2008, 44(4), 457-460.

3. Sergey F. Yan, Vladimir N. Belov, Mariano L. Bossi, Stefan W. Hell, Switchable Fluorescent and Solvatochromic Molecular Probes Based on 4-Amino- $N$-methylphthalimide and a Photochromic Diarylethene, Eur. J. Org. Chem., 2008, 2531-2538.

4. Svetlana M. Polyakova, Vladimir N. Belov, Sergey F. Yan, Christian Eggeling, Christian Ringemann, Armin de Meijere, Stefan W. Hell, New GM1 Ganglioside Derivatives for Selective Single and Double Labelling of the Natural Glycosphingolipid Skeleton, Eur. J. Org. Chem., 2009, 5162-5177.

\section{Presentationen:}

1. S. F. Yan, D. V. Dar'in, P. S. Lobanov, A. A. Potekhin, "Cyclocondensation of ethyl-3,3diaminoacrylate with ortho-chloroketones and ortho-chloronitriles from the pyrimidine series" at the International Symposium "Advanced Science in Organic Chemistry" (26-30 June, 2006, Sudak, Crimea, Ukraine);

2. S. F. Yan, V. N. Belov, M. L. Bossi, A. de Meijere, S. W. Hell, "Switchable Fluorescent Molecular Probes Based on 4-Amino- $N$-methylphthalimide and a Photochromic Diarylethene" at the "Fruehjahrssymposium 2008" (26-29 March, 2008, Rostock, Germany); 3. Svetlana Polyakova, Vladimir N. Belov, Christian Eggeling, Sergey Yan, Mariano L. Bossi, Armin de Meijere, Stefan W. Hell, "Synthesis of the GM1 ganglioside with $\omega$-aminoand $\omega$-mercaptostearic acid residues and its conjugation with photostable fluorophores" at the "Fruehjahressymposium 2008" (26-29 March, 2008, Rostock, Germany);

4. S. F. Yan, V. N. Belov, M. L. Bossi, A. de Meijere, S. W. Hell, "Towards Photoactivable Xanthene-Based Crown Ethers as Calcium Biosensors" at the International Conference on Chemistry "Main Trends of Chemistry at the Beginning of XXI Century" (21-24 April, 2009, Saint-Petersburg, Russia)

5. P. S. Lobanov, D. V. Dar'in, A. V. Galenko, S. G. Ryazanov, I. I. Eliseev, S. F. Yan, "Reaction of Heterocyclisation with Participation of Acetamidine and Acetamideoxime, which includes Electronwithdrawing group", at the International Conference on Chemistry "Main Trends of Chemistry at the Beginning of XXI Century" (21-24 April, 2009, SaintPetersburg, Russia) 